(C) 2004 by Trevor Neil Vickey. All rights reserved. 


\title{
MEASUREMENT OF $W$ BOSON POLARIZATION IN TOP QUARK DECAY
}

\author{
BY \\ TREVOR NEIL VICKEY \\ B.S., University of Illinois at Urbana-Champaign, 1998 \\ M.S., University of Illinois at Urbana-Champaign, 2000

\section{DISSERTATION} \\ Submitted in partial fulfillment of the requirements \\ for the degree of Doctor of Philosophy in Physics \\ in the Graduate College of the \\ University of Illinois at Urbana-Champaign, 2004
}

Urbana, Illinois 


\section{MEASUREMENT OF $W$ BOSON POLARIZATION \\ IN TOP QUARK DECAY}

\section{Trevor Neil Vickey, Ph.D.}

Department of Physics

University of Illinois at Urbana-Champaign, 2004

Professor Steven Errede and Professor Tony Liss, advisors

A measurement of the polarization of the $W$ boson from top quark decay is an excellent test of the $V-A$ form of the charged-current weak interaction in the standard model. Since the longitudinal $W$ boson is intimately related to the electroweak symmetry breaking mechanism, and the standard model gives a specific prediction for the fraction of longitudinal $W$ bosons from top decays, it is of particular interest for study. This thesis presents a measurement of $W$ boson polarization in top quark decays through an analysis of the $\cos \theta^{*}$ distribution in the lepton-plus-jets channel of $t \bar{t}$ candidate events from $p \bar{p}$ collisions at $\sqrt{s}=1.96 \mathrm{TeV}$. This measurement uses an integrated luminosity of $\sim 162 \mathrm{pb}^{-1}$ of data collected with the CDF Run II detector, resulting in $31 t \bar{t}$ candidate events with at least one identified $b$ jet. Using a binned likelihood fit to the $\cos \theta^{*}$ distribution from the $t \bar{t}$ candidate events found in this sample, the fraction of $W$ bosons with longitudinal polarization is determined to be $F_{0}=0.99_{-0.35}^{+0.29}$ (stat.) \pm 0.19 (syst.), $F_{0}>0.33 @ 95 \%$ CL. This result is consistent with the standard model prediction, given a top quark mass of $174.3 \mathrm{GeV} / \mathrm{c}^{2}$, of $F_{0}=0.701 \pm 0.012$. 
In memory of my dear friend, Stephen McTygue. 


\section{Acknowledgments}

I would like to begin by acknowledging all of those who have helped and guided me during my time at the University of Illinois. Two professors at the university deserve special thanks since they have had the most impact on my graduate career. I would like to thank my advisor Steven Errede for inviting me to work on the CDF experiment, for selecting me to help out with the muon calibration system, and for going to bat for me as early as the sonoluminescence days. I would also like to thank Tony Liss, my other advisor, for his eagerness to help me with a top quark analysis at CDF and for his seemingly endless suggestions.

Thank you to postdoctoral research assistants Prem Singh, Hyunsoo Kim, and Lucio Cerrito. To Prem, thank you for the buffet lunches and your help testing so many of the COT wireplanes. A special thanks to Hyunsoo Kim for being such a quiet office mate who could quickly answer any of my questions, for his unbridled willingness to help with CMP detector maintenance and of course for suggesting that I attend the SLAC Summer Institute where I met my future wife. To Lucio Cerrito, thank you for accepting the position as the CMP postdoc so that I could begin my analysis in earnest.

In addition, there are numerous other professors, postdocs, graduate students, and staff members at the University of Illinois who I would like to thank. Thank you to Tim Stelzer and Fabio Maltoni for providing me with a suitable signal Monte Carlo generator. Kevin Lannon taught me how to program computers in exchange for learning how to solder, and spent a great deal of his time pulling cables and testing 
hardware with me to get the muon calibration system and the CMP detector ready for Run II. Thanks to Shirley Rothermel, Donna Guzy and Joyce Ruch for helping with travel arrangements and paperwork. Thanks to Tom Shaw for always ensuring that I received prompt reimbursement for travel, and for writing me a personal check when my advisor forgot to sign the appointment form one semester.

This thesis would not have been possible without the dedicated effort of the hundreds of professors, postdocs, graduate students, engineers and technicians who make up the CDF Collaboration (Appendix A). I am especially thankful for the work of the top physics and lepton-plus-jets groups, in particular Patrizia Azzi, Ken Bloom, Jacobo Konigsberg, J. Stephen Miller, and Evelyn Thomson, all of whom helped to pave the way for top physics at CDF in Run II. Thank you to Erik Brubaker, Nathan Goldschmidt and Ben Kilminster for their assistance with various aspects of this analysis. A special thanks to honorary CDF collaborator Steve Mrenna, who created the MadEvent to Pythia interface and spent several long hours bug hunting with me one afternoon.

Thank you to my graduate school friends who provided ample distraction and comic relief along the way. Special thanks to Dan Finkenstadt, Paul Melby and David Posson for being great friends and for having the courage to eat my Indian cooking. Dan, thanks for staying up with me to watch the sunrise from the windows of the Seitz Overpass while slaving over Fradkin's QFT problem sets. Paul, thanks for backpacking with me and finishing the upper loop of the Zaleski trail despite the fact that it was too dark to see.

Prior to graduate school, there were those who helped, guided and distracted me during my undergraduate days. In particular, I would like to thank Greg Goodlander, Mark Neahring and Carl Salk. To Greg, thank you for believing in me before anyone else did. Mark, thanks for letting me sleep on your couch for four months. Carl, thank you for lending me money when it was desperately needed and more recently 
for being my climbing buddy-you have a standing invitation to come mountaineering in the Swiss Alps. To my uncle Mike Vickey, thank you for sending me food and money when it was needed above all else.

I would like to thank my family for their idle curiosity regarding what exactly it is that I study. Special thanks to my parents William and Kathy, who always had the best intentions and taught me that nothing is impossible. Thank you to Jennifer, Brett and my brother-in-law Cary for always being there and your willingness to listen. Thank you to my Grandmother Kipp and my Grandfather Vickey for their love and occasional financial support on my birthday and around the holidays. Thank you to my in-laws Doru and Ioana Boeriu for their instantaneous acceptance of me into their family.

I would especially like to thank my wife, Oana Boeriu, for her unwavering support and understanding. She never complained those nights that I was in my office until 3:00 am.

This thesis was supported in part by the United States Department of Energy under grant DE FG02 91ER40677. 


\section{Table of Contents}

List of Figures ..................... xi

List of Tables $\ldots \ldots \ldots \ldots \ldots \ldots \ldots \ldots \ldots \ldots \ldots \ldots$

Chapter 1 The Standard Model and the Top Quark . . . . . . 1

1.1 Introduction . . . . . . . . . . . . . . . . . 1

1.2 The Standard Model . . . . . . . . . . . . . . . . . . . . . 2

1.2.1 Quarks, Leptons and Gauge Particles . . . . . . . . . . 3

1.3 The Top Quark . . . . . . . . . . . . . . . . . . 5

1.3.1 The Weak Interaction . . . . . . . . . . . . . . 7

1.3.2 W Boson polarization in top quark decay . . . . . . . . 10

1.4 Techniques for the measurement of $W$ boson polarization . . . . . . 11

1.4.1 The $\cos \theta^{*}$ Method . . . . . . . . . . . . . 15

1.4.2 The Lepton $p_{T}$ Method . . . . . . . . . . . . . 17

Chapter 2 The Fermilab Tevatron and the CDF II Detector . . . 24

2.1 The Tevatron . . . . . . . . . . . . . . . . 24

2.2 The CDF II Detector . . . . . . . . . . . . . . . . . 28

2.2 .1 Tracking Systems . . . . . . . . . . . . . . . 31

2.2 .2 Calorimetry . . . . . . . . . . . . . . . . . . . 37

2.2 .3 Muon Detectors . . . . . . . . . . . . . . . . . . . . . . 39

2.2 .4 Trigger System . . . . . . . . . . . . . . . . . 41

Chapter $3 t \bar{t}$ Event Selection $\ldots \ldots \ldots \ldots \ldots \ldots$

3.1 Inclusive High- $p_{T}$ Electrons $\ldots \ldots \ldots \ldots \ldots \ldots$

3.1 .1 Electron Triggers . . . . . . . . . . . . . . . . 46

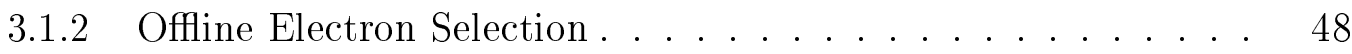

3.2 Inclusive High- $p_{T}$ Muons $\ldots \ldots \ldots \ldots \ldots \ldots \ldots \ldots \ldots \ldots \ldots$

3.2 .1 Muon Triggers . . . . . . . . . . . . . . . . . . . 52

3.2 .2 Offline Muon Selection . . . . . . . . . . . . . . 53

3.3 Jet Selection . . . . . . . . . . . . . . . . . . . . . . . 57

3.4 Secondary Vertex $b$-Tagging $\ldots \ldots \ldots \ldots \ldots \ldots$

3.5 Missing Transverse Energy . . . . . . . . . . . . . . . . . . . . 59

3.6 Primary Vertex . . . . . . . . . . . . . . . . . . . . . 60

3.7 The Lepton-plus-Jets Event Selection . . . . . . . . . . . . . . . 62 


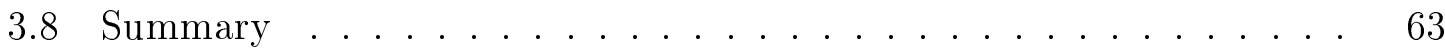

Chapter 4 Measurement Method ................665

4.1 Event Reconstruction . . . . . . . . . . . . . . . . . . . . 65

4.2 The Binned Likelihood Fitter . . . . . . . . . . . . . 68

4.2 .1 Acceptance Bias . . . . . . . . . . . . . . . . . . 68

4.2.2 The Likelihood Function . . . . . . . . . . . . . . . . 73

4.3 Kinematic Fitter Optimization Studies . . . . . . . . . . . . 73

4.3.1 Verification of the Likelihood Technique . . . . . . . 75

Chapter 5 Models of Signal and Background . . . . . . . . . 78

5.1 Models of Signal . . . . . . . . . . . . . . . . . 80

5.2 Models of Backgrounds . . . . . . . . . . . . . . . . 81

5.2 .1 Non- $W$ QCD Backgrounds . . . . . . . . . . . . . . . 81

5.2 .2 Mistag Backgrounds . . . . . . . . . . . . . . . . . 84

$5.2 .3 W$ plus Heavy Flavor Backgrounds . . . . . . . . . 85

5.2 .4 Electroweak Backgrounds . . . . . . . . . . . . . . 88

5.2 .5 Background Summary . . . . . . . . . . . . . . . 89

Chapter 6 Results................... 93

Chapter 7 Systematic Uncertainties . . . . . . . . . . . . 95

7.1 Top Mass Uncertainty . . . . . . . . . . . . . . . . . . . 96

7.2 Jet Energy Scale . . . . . . . . . . . . . . . . . . . . . . 98

7.3 Background Shape . . . . . . . . . . . . . . . . . . . 103

7.4 Gluon Radiation . . . . . . . . . . . . . . . . . . . . . . . . . . . 104

7.4.1 Initial State Radiation . . . . . . . . . . . . . . . . . 105

7.4.2 Final State Radiation . . . . . . . . . . . . . . . 106

7.5 Parton Distribution Functions . . . . . . . . . . . . . . . . . 106

7.6 Secondary Vertex Tagging . . . . . . . . . . . . . . . . . . . 107

$7.6 .1 \quad b$-tagging Efficiency . . . . . . . . . . . . . . . 107

$7.6 .2 \quad b$-tagging Scale Factor . . . . . . . . . . . . . . . . 108

7.7 Monte Carlo Modeling . . . . . . . . . . . . . . . . . . . . . . 108

7.8 Acceptance Bias Correction . . . . . . . . . . . . . . . . . . . 109

7.9 Monte Carlo Statistics . . . . . . . . . . . . . . . . . . . . 109

7.10 Summary of Systematic Uncertainties . . . . . . . . . . . . . . 110

Chapter 8 Confidence Interval Estimation $\ldots \ldots \ldots \ldots \ldots$

Chapter 9 Conclusions .................. 119

Appendix A The CDF Collaboration ............. 121 
Appendix B COT Wireplane Testing . . . . . . . . . . 128

B.1 Tension Measurements . . . . . . . . . . . . . . . . . . . . 128

B.2 Dark Current Measurements . . . . . . . . . . . . . . . 132

B.3 Source Runs . . . . . . . . . . . . . . . . . . . 136

Appendix C The Muon Calibration System . . . . . . . . 140

C.1 Chamber Calibration Hardware . . . . . . . . . . . . . . . . . . 143

C.2 Scintillator Calibration Hardware . . . . . . . . . . . . . . . 144

C.3 Calibration System Software . . . . . . . . . . . . . . . . 146

Appendix D Signal Monte Carlo Event Generators . . . . . . . . 148

D.1 Changing the random number seed . . . . . . . . . . . 150

D.2 Correcting for the systematic sampling of phase-space . . . . . 150

D.3 Changing particle IDs . . . . . . . . . . . . . . . . . . . . . 151

D.4 Adding parent particles . . . . . . . . . . . . . . . . . . 152

D.5 Fragmentation and hadronization . . . . . . . . . . . 153

D.6 Conversion from .stdhep to .hepg . . . . . . . . . . . . 153

D.7 Detector simulation . . . . . . . . . . . . . . . . . . . . . 154

Appendix E The Mistag Matrix ............... 155

E.1 Data Samples . . . . . . . . . . . . . . . . . . . . . . 156

E.2 The Secondary Vertex Tagger . . . . . . . . . . . . . . . 157

E.3 Five-Dimensional Tag Rate Matrix _. . . . . . . . . . . . . . 157

E.4 Cross-checks on the Tag-Rate Matrix . . . . . . . . . . . 160

E.5 Tag-Rate Matrix Systematic Uncertainties . . . . . . . . . . . . . 163

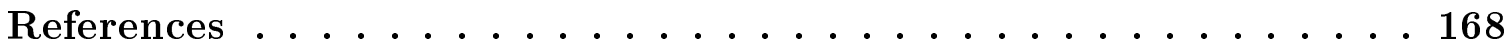

Author's Biography . . . . . . . . . . . . . . 174 


\section{List of Figures}

Figure

Page

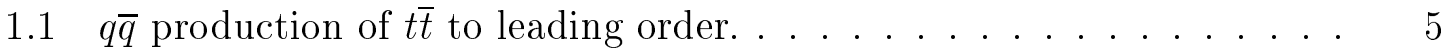

$1.2 \mathrm{gg}$ production of $t \bar{t}$ to leading order. . . . . . . . . . . . 6

1.3 (a) Charged-current weak decay of the top quark. (b) $W$ boson decay to leptons: $\bar{l}=\bar{e}, \bar{\mu}, \bar{\tau}$ and $\nu_{e}, \nu_{\mu}, \nu_{\tau}$, or Cabibbo-favored hadronic decays. . . . . . . . . . . . . . . . . .

1.4 Figures depicting total angular momentum along the $z$-axis after $W^{+}$ and $t$ decay. (a) $W^{+}$decays into a left-handed neutrino and a right handed positron. The sum of angular momentum along the $z$-axis in Figures (b) and (c) equals $+\frac{1}{2}$, identical to the angular momentum of the parent top quark along that axis, hence these decay modes are allowed. (d) Conservation of total angular momentum forbids the decay of $t$ into a left-handed $b$ and a right-handed $W^{+} \ldots \ldots \ldots \ldots$

1.5 The $W$ rest-frame. The angle between the charged-lepton momentum in the $W$ rest-frame and the $W$ momentum in the top rest-frame is defined as the angle $\theta^{*} \ldots \ldots \ldots \ldots \ldots$

1.6 Left: The true $\cos \theta^{*}$ distribution (Equation 1.12) for left-handed $W$ bosons (dotted line), longitudinal $W \mathrm{~s}$ (dashed line), and right-handed $W$ s (dash-dotted line). Right: The solid line indicates the standard model $\cos \theta^{*}$ distribution which is roughly a $70 \% / 30 \%$ split between longitudinal (dashed) and left-handed (dotted) helicity Ws. The contribution from right-handed $W \mathrm{~s}$ is negligible in the standard model $(<0.1 \%[10]) \ldots \ldots \ldots \ldots \ldots \ldots \ldots \ldots$

1.7 The true $\cos \theta^{*}$ distribution (Equation 1.12) at the generator-level (without detector simulation) for left-handed (blue, left), longitudinal (black, center) and right-handed (red, right) $W$ bosons. . . . . . . . .

1.8 The $\cos \theta^{*}$ distribution from the $m_{l b}$ approximation (Equation 1.13, using the momenta of the correct $b$ ) at the generator-level (without detector simulation) for left-handed (blue, left), longitudinal (black, center) and right-handed (red, right) $W$ bosons. . . . . . . . . .

1.9 The $\cos \theta^{*}$ distribution from the $m_{l b}$ approximation (Equation 1.13, using the momenta of the incorrect $b$ ) at the generator-level (without detector simulation) for left-handed (blue, left), longitudinal (black, center) and right-handed (red, right) $W$ bosons. . . . . . . . . . . 
1.10 The expected precision of measurements of the longitudinal fraction, $F_{0}$, when running pseudo-experiments at the generator level (without detector simulation) fitted to templates created using the $m_{l b}$ approximation to obtain the $\cos \theta^{*}$ distributions (Equation 1.13). The results shown are for pseudo-experiments with 14 different values of $F_{0}$ between 0.05 and 0.70 , in steps of 0.05 . It is clear from this plot that using the correct $b$ in the $m_{l b}$ approximation yields higher precision (black triangle) than for templates where $\cos \theta^{*}$ was calculated using the incorrect $b$ (red square). . . . . . . . . . . . . .

1.11 The lepton $p_{T}$ spectrum at the generator-level (without detector simulation) from the decay of left-handed (blue, left), longitudinal (black, center) and right-handed (red, right) $W$ bosons. . . . . . . . . . .

2.1 The Fermilab Accelerator chain [36]. . . . . . . . . . . . 27

2.2 Elevation view of one half of the CDF II detector [38]. . . . . . . . 30

2.3 A cross-sectional view of the most central regions of the CDF II detector with $\eta$ superimposed [38]. . . . . . . . . . . . .

2.4 Cross section of a CMU module showing radial alignment of alternate wires. The time difference is used at the trigger level to obtain a crude momentum measurement. The module subtends $5^{\circ}$ in $\phi$. . . . . . . .

2.5 A block diagram of the CDF II data flow [38]. This flow diagram depicts a storage pipeline 42 clock cycles deep (for a 132 ns clock cycle and a $7.6 \mathrm{MHz}$ bunch crossing rate). The current configuration of the Tevatron delivers events to CDF at a rate of $2.5 \mathrm{MHz}$, which is a 396 ns clock cycle; with this configuration the Level-1 pipeline is capable of storing 14 clock cycles. . . . . . . . . . . . . . .

4.1 Top figure: The jet response, $\left(p_{T}^{\text {parton }}-p_{T}^{\text {jet }}\right) / p_{T}^{\text {jet }}$, as a function of $p_{T}^{\text {jet }}$ for $b$-jets and $W$-jets after only generic corrections are applied. Bottom figure: The jet response as a function of $\eta^{\text {jet }}$ after applying $t \bar{t}$-specific corrections that depend only on $p_{T}^{\text {jet }}$. Both of these figures are taken from [64], which details a measurement of the top quark mass at CDF during Run II. . . . . . . . . . . . . . . . . .

4.2 Top figure: The invariant mass of jets from $W$ daughters as a function of $p_{T}^{W}$ after applying generic corrections (full circles) and $p_{T}$ and $\eta$ dependent corrections (open circles). Bottom figure: The invariant mass of jets from $W$ daughters as a function of the average $\eta$ of $W$ jets after applying only $p_{T}$-dependent corrections (full circles) and $p_{T}$ and $\eta$ dependent corrections (open circles). In both figures, MC events with a good jet-parton match are used. Both of these figures are taken from [64], which details a measurement of the top quark mass at CDF during Run II. . . . . . . . . . . . . . . . . .

4.3 The results of signal and background acceptance studies for the free and the constrained fits from the top mass fitter. . . . . . . . . 
4.4 A Gaussian fit to the distribution of $F_{0}^{o b s}$ measured values for $10 \mathrm{k}$ pseudo-experiments where the constrained fit and a $\chi_{\min }^{2}<20$ cut were used. . . . . . . . . . . . . . . . .

4.5 A Gaussian fit to the pull distribution for $10 \mathrm{k}$ pseudo-experiments where the constrained fit and a $\chi_{\min }^{2}<20$ cut were used. We define the pull as $\left(F_{0}^{\text {meas. }}-F_{0}^{a c t u a l}\right) / \delta F_{0}^{\text {meas. }} \ldots \ldots . . . \ldots$

5.1 Binned signal templates used in the $\cos \theta^{*}$ analysis. The left-handed signal template is shown on the left, and the longitudinal signal template is to the right. As discussed in Chapter $1, \cos \theta^{*}$ is calculated from the $m_{l b}$ approximation and when $m_{l b}>m_{t}^{2}-M_{W}^{2}$ we can obtain values of $\cos \theta^{*}>1 \ldots \ldots \ldots \ldots$

5.2 A comparison of the $W c \bar{c}$ and $W b \bar{b}$ shapes from Monte Carlo data. Due to this agreement, and the low statistics of the $W c \bar{c} \mathrm{MC}$ sample, the $W b \bar{b} \cos \theta^{*}$ distribution is used to model the $W c \bar{c}$ shape. . . . . .

5.3 A comparison of the $W c$ and $W b \bar{b}$ shapes from Monte Carlo data. Due to this agreement, and the low statistics of the $W c \mathrm{MC}$ sample, the $W b \bar{b} \cos \theta^{*}$ distribution is used to model the $W c$ shape. . . . . . . . .

5.4 The sum of individual contributions to the background $\cos \theta^{*}$ template is obtained from data and Monte Carlo events. Data is used to model the non- $W$ QCD background and Monte Carlo simulation is used to model the Mistag, Heavy Flavor + EWK (modeled using $W b \bar{b}$ ), and single top backgrounds. The normalizations are taken from Table 5.3.

6.1 The $\cos \theta^{*}$ distribution for the lepton-plus-jets sample with signal and background templates normalized according to their Maximum Likelihood Estimators (MLEs) superimposed. Inset is the projection of negative log-likelihood along the $F_{0}$ axis for the fit to the data. As discussed in Chapter $1, \cos \theta^{*}$ is calculated from the $m_{l b}$ approximation and when $m_{l b}^{2}>m_{t}^{2}-M_{W}^{2}$ it is possible to obtain values of $\cos \theta^{*}>1$. Values of $\cos \theta^{*}<-1$ are not possible since $m_{l b}^{2} \nless 0$. . . . . . . .

7.1 Gaussian fits to distributions of $10 \mathrm{k} F_{0}$ values obtained from the likelihood fitter by running pseudo-experiments. The black solid line shows the Gaussian fit to results from pseudo-experiments with $m_{t}=175$ $\mathrm{GeV} / \mathrm{c}^{2}$. The blue dashed line shows the Gaussian fit to results from pseudo-experiments with $m_{t}=180 \mathrm{GeV} / \mathrm{c}^{2}$. The red dash-dotted line shows the Gaussian fit to results from pseudo-experiments with $m_{t}=170 \mathrm{GeV} / \mathrm{c}^{2}$. The systematic uncertainty due to the top mass uncertainty is taken to be $0.08 . \ldots \ldots \ldots$ 
7.2 Gaussian fits to ditributions of $10 \mathrm{k} F_{0}$ values obtained from the likelihood fitter by running multiple sets of pseudo-experiments where the background shape is from one specific source (e.g., Single-top schannel), or a particular combination of sources (e.g., the default shapes and normalizations but with twice the number of non- $W$ QCD events). I assign a systematic uncertainty due to the background shape equal to the absolute value of the largest deviation from the default mean,

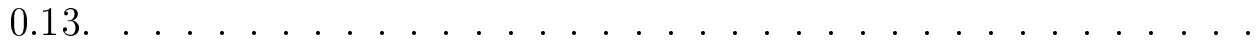

7.3 Plots taken from [84] which show tag efficiencies as a function of the parameter $b \Delta \epsilon$ for events reconstructed as lepton plus jets events with $\geq$ four jets. $b$ is the branching fraction for $t \rightarrow W b(\sim 1$ in the standard model), and $\Delta \epsilon=\epsilon_{b}-\epsilon_{q}$ where $\epsilon_{q}$ is the probability of tagging a light quark [84, 85]. The solid lines denotes tag efficiencies as a function of $b \Delta \epsilon$, the dashed lines denote tag efficiencies as a function of $b \Delta \epsilon$ assuming that $\epsilon_{c}=\epsilon_{q}=0 \ldots \ldots \ldots$. . . . . . . .

7.4 Gaussian fits to distributions of $10 \mathrm{k} F_{0}$ values obtained from the likelihood fitter by running pseudo-experiments where the signal is modeled by PYTHIA (red dash-dotted line) or HERWIG (blue dashed line) Monte Carlo programs. The black vertical line shows the mean value from a Gaussian fit to results from pseudo-experiments using MadEvent Monte Carlo to model the signal where $F_{0}=0.70$. Because of this shift, I assign a systematic uncertainty due to Monte Carlo Modeling equal to the absolute value of the largest deviation from the vertical line, 0.04.

8.1 $1 \sigma, 2 \sigma$ and $3 \sigma$ Feldman-Cousins confidence intervals for an experiment in the lepton-plus-jets sample. These intervals include systematic uncertainties. The thick vertical line at $\hat{F}_{0}=0.99$ indicates the experimental outcome. ................... . . . . .

8.2 Parameterization of $\mu\left(F_{0}\right)$ for pseudo-experiments using the default binned likelihood fitter. The best fit to the data points with a firstorder polynomial is drawn black, with $p_{0}$ and $p_{1}$ denoting the polynomial coefficients. For comparison, a line with unit slope and $y$-intercept of zero is shown in red. . . . . . . . . . . . . . . .

8.3 Parameterization of $\sigma\left(F_{0}\right)$ for pseudo-experiments using the default binned likelihood fitter. A black line denotes the best fit to the data points; the functional form is that of a second-order polynomial. . . .

B.1 Wireplane tension testing setup [88]. . . . . . . . . . . . 131

B.2 A plot of the driving frequency versus the out-of-phase voltage. . . . 132

B.3 The average tension (in grams) for each wireplane, plotted by superlayer number. . . . . . . . . . . . . . . . . . 133

B.4 A schematic of the four-wireplane high-voltage test box. . . . . . . 134

B.5 A schematic of the data acquisition setup for dark current and source

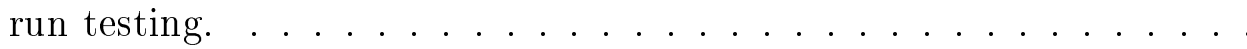


B.6 The distribution of the ratio of the dark currents measured at nominal voltage (denominator) and nominal+200 V (numerator). . . . . . 136

B.7 Current measurements for one wireplane during a 9.5 hour source run. 138

C.1 A schematic view of a CMP/CMX drift chamber. Note that the PVC wire supports are used only for the CMP chambers. . . . . . . . . . 142

C.2 The calibration hardware setup for the CDF II CMP, CMX and BMU drift chamber muon systems. . . . . . . . . . . . . . . . . . . 145

C.3 The calibration hardware setup for the CDF II CSP, CSX, BSU and TSU scintillator muon systems. . . . . . . . . . . . . . . . . 146

E.1 $+L_{x y}$ is the distance between the primary vertex and the secondary vertex. The impact parameter, $d_{0}$, is the distance of closest approach of a track to the primary vertex. . . . . . . . . . . . 156

E.2 Positive and negative tag rates in the combined jet sample as a function of $E_{T}$ (top) and taggable track multiplicity (bottom). . . . . . . 158

E.3 Number of observed (triangle) and predicted (circle) positive and negative tags in the $\Sigma E_{T}$ sample binned as a function of the number of jets in the event. . . . . . . . . . . . .

E.4 Number of observed (triangle) and predicted (circle) positive and negative tags in the $\Sigma E_{T}$ sample binned as a function of the number of vertices in the event. . . . . . . . . . . . . . .

E.5 Number of observed (triangle) and predicted (circle) positive and negative tags in the $\Sigma E_{T}$ sample binned as a function of the $\Sigma E_{T}$ of the

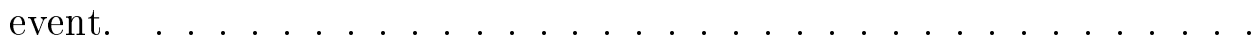

E.6 Number of observed (triangle) and predicted (circle) positive and negative tags in the $\Sigma E_{T}$ sample binned as a function of instantaneous luminosity. . . . . . . . . . . . . . . . . . . . 166

E.7 Number of observed (triangle) and predicted (circle) positive and negative tags in the $\Sigma E_{T}$ sample binned as a function of run number. . . 167 


\section{List of Tables}

Table

Page

1.1 Symbols, charges and masses of gauge bosons in the standard model. Bosons have integer spin; all of the listed in this table are spin 1 with the exception of the graviton which is spin 2 . The mass values shown here are taken from the PDG review of particle physics [2]. ${ }^{\dagger}$ Theoretical value; a mass of even a few $\mathrm{MeV} / \mathrm{c}^{2}$ may not be precluded. $\ddagger$ Gravitational waves (and their quanta) have yet to be discovered by

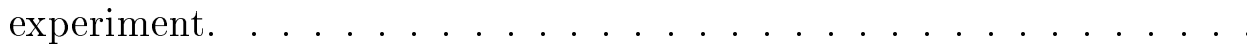

1.2 Mass and charge of the quarks and leptons in the standard model. The masses are not predicted by the standard model; the values shown here reflect measurements made by experiment. Quarks and leptons are fermions, and fermions have half-integer spin; all of the fermions listed here are believed to be spin $\frac{1}{2}$. Every quark and lepton has an antimatter counterpart (e.g. anti-top, $\bar{t}$, is the antimatter counterpart to the top quark, $t) . \ldots \ldots \ldots \ldots$

2.1 Machines and corresponding energies at the Fermilab accelerator com-

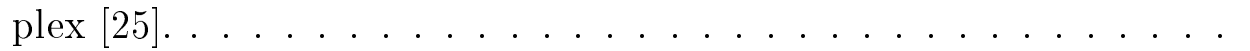

3.1 Identification and kinematic cuts for electrons, muons and jets. . . . .

4.1 The results of various methods attepted to match jets to the correct $b$-parton. Percentages shown reflect the fraction of the time that each method matches a jet to the correct $b$-parton. . . . . . . . . .

4.2 The results of pseudo-experiments carried out for optimization studies. The uncertainty on the uncertainties are not shown, but are about \pm 0.02 . 75

5.1 Background estimates in the 3.5 and $\geq 4$-jet bins. These numbers do not include the acceptance factors due to the $\chi_{\text {min }}^{2}<20$ cut (see Table 5.2). . . . . . . . . . . . . . . .

5.2 Acceptance factors in the 3.5 and $\geq 4$-jet bins. These numbers come from running the event selection criteria (including the $\chi_{\min }^{2}<20 \mathrm{cut}$ ) on Monte Carlo events, or in the case of the non- $W$ QCD contribution, data. Note that the diboson and $Z \rightarrow \tau \tau$ acceptance numbers have been estimated using the heavy flavor Monte Carlo events (the table entry corresponding to "Heavy Flavor + EWK"). . . . . . . . . 
5.3 Background estimates in the 3.5 and $\geq 4$-jet bins calculated using the pre-acceptance background estimates from Table 5.1 and the acceptance factor due to the $\chi_{\min }^{2}<20$ cut (Table 5.2). Note that the table entry for "Heavy Flavor + EWK" includes the background estimates for the diboson and $Z \rightarrow \tau \tau$ processes. For the 31 events which pass the selection criteria, the fraction of background events is determined to be $\beta_{0}=0.22 \pm 0.03 \ldots \ldots \ldots \ldots$

7.1 The fraction of longitudinal $W$ bosons for various top quark masses, as predicted by the standard model. The uncertainty in the longitudinal fraction for $m_{t}=174.3 \mathrm{GeV} / \mathrm{c}^{2}$ reflects the propagation of error on the top and $W$ masses. The $W$ mass used to obtain the numbers in the above table is $80.423 \pm 0.039 \mathrm{GeV} / \mathrm{c}^{2}[2] \ldots \ldots$. . . . . . .

7.2 Uncertainties associated with relative corrections for various regions of

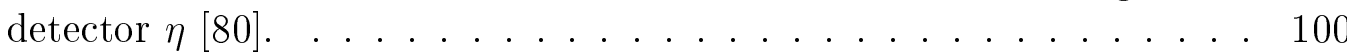

7.3 Systematics for the $W$ boson polarization $\cos \theta^{*}$ analysis. . . . . . . . 110

7.4 $\Delta F_{0}$ as determined by taking the difference between the mean $F_{0}$ measured with default shapes for $10 \mathrm{k}$ pseudo-experiments and that measured by shifting each systematic source by $\pm 1 \sigma$. ${ }^{\dagger}$ Numbers for the $\mathrm{PDF} \Delta F_{0}$ denote the absolute difference. ${ }^{\ddagger}$ MadEvent was used for $t \bar{t}$ generation and PYTHIA was used for hadronization and fragmentation.

9.1 Projections for the statistical uncertainty on the measured longitudinal fraction, using the $\cos \theta^{*}$ technique, for various integrated luminosity values. . . . . . . . . . . . . . . . . . .

B.1 COT wireplane lengths, in inches, for each superlayer [86]. . . . . .

E.1 Tag rate matrix variables and bin boundries. The numbers shown are the lower bin boundaries. . . . . . . . . . . . . . .

E.2 Predicted vs. observed number of tags for trigger jets in each of the 4.8.4 (with 4.9.1 b-tagging) datasets. This cross-check was done against the five-dimensional tag-rate matrix. . . . . . . . . . .

E.3 Predicted vs. observed number of tags for non-trigger jets in each of the 4.8.4 (with 4.9 .1 b-tagging) datasets. This cross-check was done against the five-dimensional tag-rate matrix. . . . . . . . . . .

E.4 Predicted vs. observed number of tags for the five-dimensional tag-rate matrix in each of the samples considered. . . . . . . . . . . . 163

E.5 Systematic uncertainties for the Five-dimensional SecVtx tag-rate matrix . . . . . . . . . . . . . . . . . . 


\section{Chapter 1}

\section{The Standard Model and the Top Quark}

\section{$1.1 \quad$ Introduction}

Physicists strive to understand the world in a quantitative manner, developing theories and conjectures to explain experimental measurements. Particle physics is the study of the most fundamental constituents of all matter and their interactions. The most successful theory of particle physics today, known as the "standard model", was developed in the 1970s and incorporated all of the particles and interactions that were known at that time. Since the conception of this theory, it has successfully predicted the outcome of a large variety of experiments (including the existence of the top quark). As the field of particle physics continues to push the boundary of the high-energy frontier, we further test our understanding of the most fundamental aspects of nature. Somewhere in this unexplored territory is a chance to disprove the standard model-that opportunity may lie with the top quark and its properties.

The remainder of this Chapter describes the standard model and introduces the experiment carried out by this thesis as a test of the standard model. Since this thesis presents a measurement of top quark decay properties, this first chapter also focuses on top quark physics and the weak interaction. Chapter 2 describes the experimental apparatus, Chapter 3 details the event selection for selecting events in this dataset, 


\begin{tabular}{|cccc|}
\hline \hline Force & Particle (symbol) & Charge $(e)$ & Mass $\left(\mathrm{GeV} / \mathrm{c}^{2}\right)$ \\
\hline Strong & Gluon $(g)$ & 0 & $0^{\dagger}$ \\
Electromagnetic & Photon $(\gamma)$ & 0 & 0 \\
Weak (charged) & $W$ Boson $\left(W^{ \pm}\right)$ & \pm 1 & $80.425 \pm 0.038$ \\
Weak (neutral) & $Z$ Boson $(Z)$ & 0 & $91.1876 \pm 0.0021$ \\
Gravitational & Graviton $^{\ddagger}(G)$ & 0 & 0 \\
\hline \hline
\end{tabular}

Table 1.1: Symbols, charges and masses of gauge bosons in the standard model. Bosons have integer spin; all of the listed in this table are spin 1 with the exception of the graviton which is spin 2. The mass values shown here are taken from the PDG review of particle physics [2]. ${ }^{\dagger}$ Theoretical value; a mass of even a few $\mathrm{MeV} / \mathrm{c}^{2}$ may not be precluded. ${ }^{\ddagger}$ Gravitational waves (and their quanta) have yet to be discovered by experiment.

the analysis method is explained in Chapter 4, the Monte Carlo programs used to model signal and background events are discussed in Chapter 5. The result of the measurement, the associated systematic uncertainties, as well as the interpretation of this result are described in Chapters 6-9. Several appendicies are placed at the end of this thesis to explain in greater detail a few aspects of the experimental apparatus and the analysis which might have caused a significant digression if placed within the main text.

\subsection{The Standard Model}

The theory describing the strong and electroweak interactions is what particle physicists refer to as the "standard model." In this theory [1], the fundamental constituents of all known matter are spin- $\frac{1}{2}$ fermions named, "quarks" and "leptons;" all interactions between the fermions are mediated by spin-1 particles known as gauge bosons (Table 1.1). Because our universe is comprised of these constituents, it is important that we understand their interactions as well as their properties. 


\subsubsection{Quarks, Leptons and Gauge Particles}

In the standard model there are three generations of matter and anti-matter. Quarks come in six different "flavors," which may be classified as two main types: "up"-type $(u)$ and "down"-type $(d)$. As a convenient idealization, ${ }^{1}$ we say that quarks may only exist in bound states, called "hadrons," ${ }^{2}$ hence there are no free quarks. Since quarks partake in both the electroweak and strong interactions, they possess electric, weak and color ("strong") charges. Leptons exist in two varieties: charged $(e, \mu, \tau)$ and neutral $\left(\nu_{e}, \nu_{\mu}, \nu_{\tau}\right)$. The neutral leptons are called "neutrinos." Leptons only participate in the electroweak interaction, and hence do not possess a color charge. For each particle, there exists an antiparticle of opposite charge but identical mass, lifetime and spin. Table 1.2 depicts the mass and charge of quarks and leptons for all three generations in the standard model. The masses of the quarks and leptons are not predicted by the standard model.

The interactions between all leptons and quarks are described by two quantum field theories. The electroweak (EW) and quantum chromodynamic (QCD) field theories govern the electroweak and strong interactions, respectively. In any quantum field theory the symmetries of the theory imply the existence of conservation laws, and the gauge fields of the theory mediate the forces it describes. Just as in quantum electrodynamics (QED), where the gauge symmetry of the theory implies the existence of a massless photon $(\gamma)$, the $S U(2) \times U(1)$ and $S U(3)$ gauge symmetries of EW and QCD lead to the presence of the $\gamma, W^{ \pm}, Z$, and $g$ gauge bosons ${ }^{3}$ in the standard model. As in QED the EW $\gamma$ is massless and mediates the electromagnetic force; unlike QED this theory also contains three massive gauge bosons which mediate the

\footnotetext{
${ }^{1}$ The top quark decays so rapidly that it does not have a chance to hadronize. So to a good approximation top decays as a "free" quark.

${ }^{2}$ The color charges of QCD are red, blue and green $(R, B, G)$. Hadrons are color-neutral bound states of quarks. There are two types of hadrons: quark anti-quark mesons and three-quark baryons.

${ }^{3}$ There are eight gluons $(g)$ in QCD. These massless gauge bosons mediate the strong force. Gluons carry color from one quark to another, and hence gluons themselves cannot be in a color singlet state; for example, a gluon could be in the state $B \bar{R}$, but not $R \bar{R}+B \bar{B}+G \bar{G}$.
} 


\begin{tabular}{|cccc|}
\hline \hline Generation & Particle (symbol) & Charge $(e)$ & Mass $\left(\mathrm{GeV} / \mathrm{c}^{2}\right)$ \\
\hline \multirow{3}{*}{ First } & Electron $(e)$ & -1 & $5.11 \times 10^{-4}$ \\
& Electron neutrino $\left(\nu_{e}\right)$ & 0 & $<3.0 \times 10^{-9}$ \\
& Up quark $(u)$ & $+2 / 3$ & $1.5-4 \times 10^{-3}$ \\
& Down quark $(d)$ & $-1 / 3$ & $4-8 \times 10^{-3}$ \\
\hline \multirow{3}{*}{ Second } & Muon $(\mu)$ & -1 & $1.06 \times 10^{-1}$ \\
& Muon neutrino $\left(\nu_{\mu}\right)$ & 0 & $<1.9 \times 10^{-4}$ \\
& Charm quark $(c)$ & $+2 / 3$ & $1.2-1.4$ \\
& Strange quark $(s)$ & $-1 / 3$ & $8.0-13 \times 10^{-2}$ \\
\hline \multirow{3}{*}{ Third } & Tau $(\tau)$ & -1 & 1.78 \\
& Tau neutrino $\left(\nu_{\tau}\right)$ & 0 & $<1.82 \times 10^{-2}$ \\
& Top quark $(t)$ & $+2 / 3$ & $174.3 \pm 5.1$ \\
& Bottom quark $(b)$ & $-1 / 3$ & $4.1-4.4$ \\
\hline \hline
\end{tabular}

Table 1.2: Mass and charge of the quarks and leptons in the standard model. The masses are not predicted by the standard model; the values shown here reflect measurements made by experiment. Quarks and leptons are fermions, and fermions have half-integer spin; all of the fermions listed here are believed to be spin $\frac{1}{2}$. Every quark and lepton has an antimatter counterpart (e.g. anti-top, $\bar{t}$, is the antimatter counterpart to the top quark, $t$ ).

weak force: the charged $W^{ \pm}$, and the electrically neutral $Z$. The masses of these three gauge bosons are acquired through the Higgs mechanism, whereby the massless scalar and vector fields of the Lagrangian $\left(\phi, \phi^{*}\right.$, and $\left.G_{\mu}\right)$ appear as the fields $\eta, G_{\mu}$, and $\xi$ after the symmetry of the Lagrangian is spontaneously broken. In this theory the massive scalar $\eta$ is known as the "Higgs boson;" the $G_{\mu}$ and $\xi$ fields are rewritten using the gauge transformation

$$
G_{\mu} \rightarrow G_{\mu}^{\prime}=G_{\mu}+\frac{1}{q v} \partial_{\mu} \xi
$$

For a massless gauge field, $A_{\mu}$, we may choose a gauge where $\vec{\nabla} \cdot \vec{A}=0$. This is the Coulomb gauge of electrodynamics. We can rewrite the Coulomb gauge in momentum space as $\vec{k} \cdot \vec{A}(\vec{k})=0$, which tells us that the field is polarized transverse to the momentum $\vec{k}$. In the Higgs mechanism, the gauge field $G_{\mu}^{\prime}$ has a longitudinal 


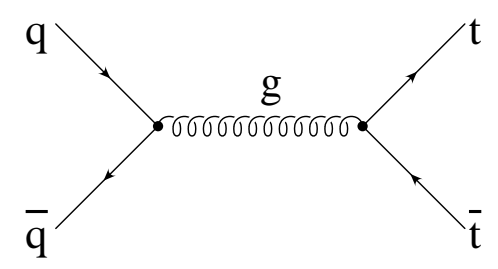

Figure 1.1: $q \bar{q}$ production of $t \bar{t}$ to leading order.

component. As can be seen by taking the divergence of the gauge field $G_{\mu}^{\prime}$

$$
\vec{\nabla} \cdot \vec{G}^{\prime}=\partial^{i} G_{i}^{\prime}=\partial^{i} G_{i}+\frac{1}{q v} \partial^{i} \partial_{i} \xi=\frac{1}{q v} \nabla^{2} \xi
$$

This means that in momentum space we have $\vec{k} \cdot \vec{G}^{\prime}(\vec{k}) \neq 0$. In other words, the field $\xi$ from the Higgs mechanism gives rise to the longitudinal component of the massive vector field $G_{\mu}^{\prime}$-this is the longitudinal $W$ boson. The relationship between electroweak symmetry breaking and the longitudinal $W$ is what makes the analysis carried out in this thesis of particular interest. Due to the large value of the top quark mass, top decay is the only significant source of longitudinal $W$ bosons.

\subsection{The Top Quark}

The discovery of the elusive top quark by the CDF [3] and D0 [4] collaborations in 1995 revealed that this was the heaviest known elementary particle. Weighing in at $\sim 175 \mathrm{GeV} / c^{2}$, nearly the mass of a gold nucleus and almost 40 times the mass of the next heaviest quark, ${ }^{4}$ it is close to the scale of electroweak symmetry breaking in the standard model. The Higgs mechanism is responsible for the top quark mass, and according to standard model theory top's Yukawa coupling, $g_{t t H}$, is $\sim 1$

$$
g_{t t H}=\frac{\sqrt{2} m_{t}}{v}=\frac{\sqrt{2} m_{t}}{246} \simeq 1 .
$$

\footnotetext{
${ }^{4}$ The next heaviest quark is the $b$ with a mass of $\sim 4 \mathrm{GeV} / c^{2}$.
} 

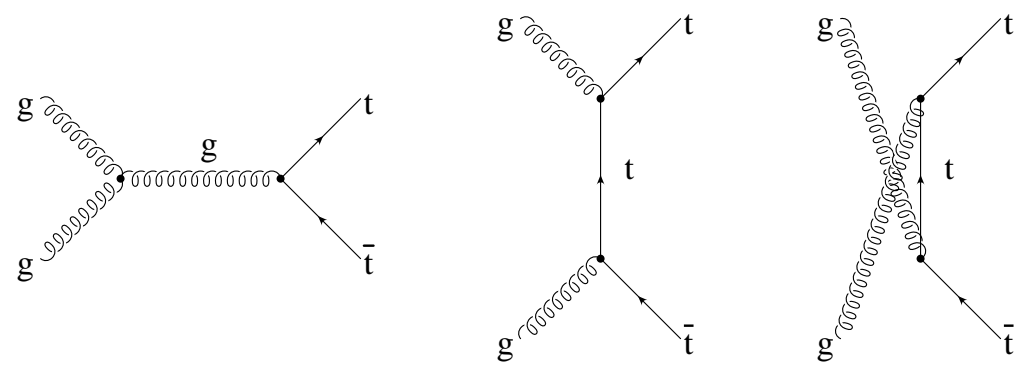

Figure 1.2: $g g$ production of $t \bar{t}$ to leading order.

Does the top quark play a role in the electroweak symmetry breaking mechanism? This is one of the most important questions in particle physics today. Precision measurements of top quark properties and comparison to standard model expectations can probe our understanding of electroweak symmetry breaking.

The top quark has a lifetime of about $0.5 \times 10^{-24} \mathrm{~s}$, much shorter than any other quark. In fact, the top quark's lifetime is less than the QCD hadronization time-scale, which means that it decays before it has a chance to hadronize. This scenario is unlike that of any of the lighter quarks, which live long enough to pair-up and form bound states resulting in the creation of mesons and baryons. Since the top quark does not hadronize, studying the top quark is about as close as one can get to studying a bare quark. Unlike in the decays of lighter quarks, where the quark spin information is lost during hadronization, the top quark spin is passed directly to it's decay products in a manner which is explicitly understood [5].

The top quark decays predominantly via the charged-current weak interaction $t \rightarrow W^{+} b\left(\right.$ and $\left.\bar{t} \rightarrow W^{-} \bar{b}\right)$. There are several other possible decays of the top quark, but they are exceptionally rare. ${ }^{5}$ Since the top quark is heavier than the combined masses of the $W$ boson $\left(\sim 80 \mathrm{GeV} / \mathrm{c}^{2}\right)$ and the $b$-quark $\left(\sim 4 \mathrm{GeV} / \mathrm{c}^{2}\right)$, it will decay into a real $W$ whereas lighter quarks will decay into a virtual $W$. Because the top

\footnotetext{
${ }^{5}$ The charged-current weak decays $t \rightarrow W^{+} d$ and $t \rightarrow W^{+} s$ are suppressed due to the small values of the Cabibbo-Kobayashi-Maskawa (CKM) matrix [6, 7] elements $\left|V_{t d}\right|$ and $\left|V_{t s}\right|$. Additionally, in the standard model the flavor-changing neutral-current modes $t \rightarrow \gamma q(q=u, c)$ and $t \rightarrow Z q(q=u, c)$ are very rare $[8,9]$.
} 


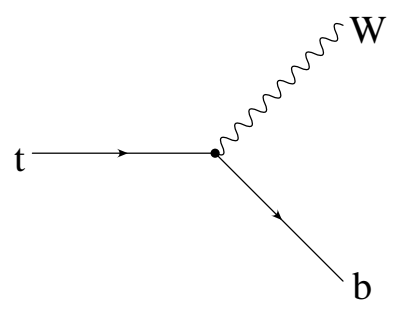

(a)

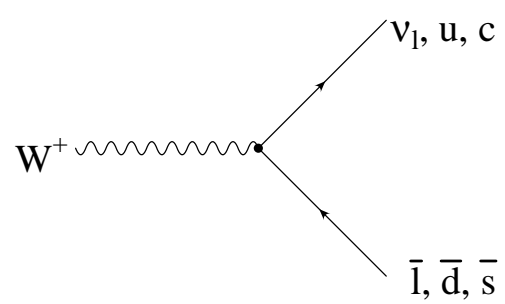

(b)

Figure 1.3: (a) Charged-current weak decay of the top quark. (b) $W$ boson decay to leptons: $\bar{l}=\bar{e}, \bar{\mu}, \bar{\tau}$ and $\nu_{e}, \nu_{\mu}, \nu_{\tau}$, or Cabibbo-favored hadronic decays.

quark is so heavy, and the other quarks are so light, top decay is the only significant source of longitudinal $W$ bosons (Equation 1.8). QCD production, the only other source of real $W$ bosons, produces nearly all transversely polarized $W$ s.

At the Tevatron, top quarks are most often produced in $t \bar{t}$ pairs via the strong interaction (see Fig. 1.1). Approximately $90 \%$ of the total $t \bar{t}$ production cross-section at the Tevatron is through quark-antiquark annihilation, the remaining fraction is from gluon-gluon fusion (see Fig. 1.2).

\subsubsection{The Weak Interaction}

The weak interaction comes in two varieties: neutral-current and charged-current interactions. Because the top quark predominantly decays via the charged-current weak interaction (Figure 1.3(a)), I will not focus on the neutral-current interaction in this thesis. The $W^{ \pm}$bosons are the mediators of the charged-current weak interaction, and the $Z$ boson is responsible for the neutral-current weak interaction.

If one considers $W^{+}$decay to leptons, the diagram of Figure 1.3(b) comes to mind. We know the Feynman rules for such a vertex, and can immediately write down:

$$
-i \frac{g}{\sqrt{2}} \gamma^{\mu} \frac{1}{2}\left(1-\gamma^{5}\right)
$$

This is what is known as the vector $\left(\bar{\psi} \gamma^{\mu} \psi\right)$ minus axial vector $\left(\bar{\psi} \gamma^{\mu} \gamma^{5} \psi\right)$, or $V-A$, 
form of the weak current. The weak interaction is not purely $V-A$. While it is believed that the charged-current interaction is purely $V-A$ (i.e., left-handed), the neutral-current weak interaction is not. We recognize $\frac{1}{2}\left(1-\gamma^{5}\right)$ as the left-handed projection $^{6}$ operator. This combination of vector and axial vector means that parity is violated, as this operator only projects out left-handed ${ }^{7}$ neutrinos (or right-handed anti-neutrinos). In other words, charged leptons are only coupled to left-handed neutrinos in the weak interaction. If we flank the mathematical expression for this vertex with two four-component spinors, one spinor with two-component objects ${ }^{8}$ representing the charged lepton $\left(\bar{u}_{l}\right)$ and the other with two-component objects representing the neutrino $\left(u_{\nu}\right)$, we find that:

$$
\bar{u}_{l} \gamma^{\mu} \frac{1}{2}\left(1-\gamma^{5}\right) u_{\nu}=\bar{u}_{l} \frac{1}{2}\left(1+\gamma^{5}\right) \gamma^{\mu} u_{\nu}
$$

where

$$
\bar{u}_{l} \frac{1}{2}\left(1+\gamma^{5}\right)=\left(\bar{u}_{l}\right)_{L}
$$

represents left-handed charged leptons. Now we can see that the $V-A$ structure of the charged weak interaction only permits leptonic $W^{+}$decay into left-handed leptons, or right-handed anti-leptons. Of course, hadronic decay of the $W^{+}$is also possible (Figure 1.3(b)) and because of the $V-A$ form a $W^{+}$boson is only able to decay into left-handed quarks and right-handed anti-quarks.

Assuming that only left-handed neutrinos are permitted in nature, we can draw the same conclusion with regards to the charged-lepton handedness simply by considering

\footnotetext{
${ }^{6} P_{ \pm}$are the left $(-)$and right $(+)$projection operators given as $\frac{1}{2}\left(1 \pm \gamma^{5}\right)$.

${ }^{7}$ The helicity operator $\vec{\sigma} \cdot \hat{p}$ acting on some state will return helicity eigenvalues. Effectively, helicity is the dot product of the spin and unit-momentum vectors for a given particle. For a spin- $\frac{1}{2}$ particle with it's spin aligned parallel to the direction of motion, it could have helicity eigenvalues of $\pm \frac{1}{2} .+\frac{1}{2}$ when the spin vector points in the direction of motion (right-handed), or $-\frac{1}{2}$ when the spin vector points in the direction opposite the motion of the particle (left-handed). In the extreme relativistic limit, the chirality operator, $\gamma^{5}$, is equal to the helicity operator. Because the $W$ is much more massive than it's decay products, the chirality of the daughters will be nearly equivalent to their helicity.

${ }^{8}$ These two two-component objects $(\bar{u})_{L}$ and $(\bar{u})_{R}$ are the left and right-handed "Weyl spinors."
} 


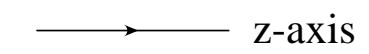

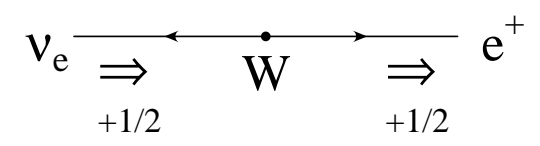

(a)

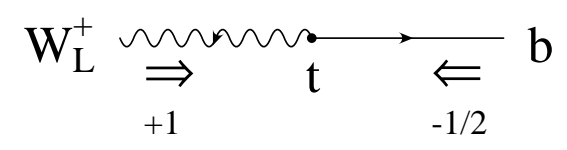

(c)

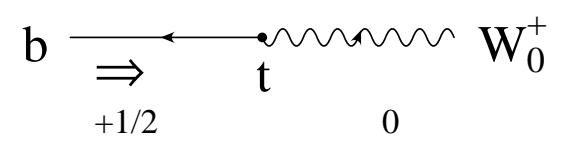

(b)

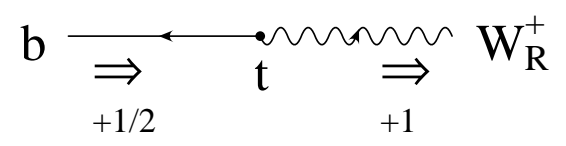

(d) Forbidden

Figure 1.4: Figures depicting total angular momentum along the $z$-axis after $W^{+}$ and $t$ decay. (a) $W^{+}$decays into a left-handed neutrino and a right handed positron. The sum of angular momentum along the $z$-axis in Figures (b) and (c) equals $+\frac{1}{2}$, identical to the angular momentum of the parent top quark along that axis, hence these decay modes are allowed. (d) Conservation of total angular momentum forbids the decay of $t$ into a left-handed $b$ and a right-handed $W^{+}$.

conservation of total angular momentum. In the $W^{+}$rest-frame, we may choose the $z$-axis such that the entire spin- 1 of the $W^{+}$points along it in the positive direction (see Figure 1.4(a)). When the $W^{+}$decays leptonically to a right-handed positron and a left-handed electron-neutrino, we find the total angular momentum along the $z$-axis is conserved. The angular momentum would not be conserved if, for example, the $W^{+}$decayed into a right-handed positron and a right-handed neutrino.

We are able to reach similar conclusions regarding the weak decay of the top quark. Using the Feynman rules, we can write down the mathematical representation of this decay vertex (Figure 1.3(a))

$$
-i \frac{g}{\sqrt{2}}\left|V_{t b}\right| \gamma^{\mu} \frac{1}{2}\left(1-\gamma^{5}\right)
$$

and note that it contains the same $V-A$ structure as the leptonic $W^{+}$decay represented by Equation 1.4. Neglecting the $b$-quark mass in comparison to that of the 
$t$-quark or the $W^{+}$, we deduce that like the neutrino previously considered, the $b$ quark in this decay must also be left-handed. We can use the same total angular momentum arguments as before, but this time we go into the top rest-frame and

choose the $z$-axis such that the spin- $\frac{1}{2}$ of top is aligned in the positive direction (see Figures 1.4(b), (c), and (d)). We conclude that of the three possible $W^{+}$helicity states, only two are realizable due to the $V-A$ structure of the weak interaction. When polarization of the $W^{+}$is completely orthogonal to it's direction of motion (helicity \pm 1 ), we denote this as $W_{R}^{+}$or $W_{L}^{+}$for the left or right helicity states and label these states "transversely polarized." 9 If the polarization of the $W^{+}$is parallel to it's direction of motion (helicity 0 state), we denote this as $W_{0}^{+}$and name it "longitudinally polarized." We can infer from Figure 1.4(c) that in top decay a transversely polarized $W^{+}$may only be left-handed, and similarly a transversely polarized $W^{-}$ may only be right-handed. Figure $1.4(\mathrm{~d})$ depicts a situation where total angular momentum is not conserved, as the $b$-quark and $W^{+}$angular momentum contributions along the $z$-axis sum to $+\frac{3}{2}$.

\subsubsection{W Boson polarization in top quark decay}

The standard model gives a specific prediction for the fraction of longitudinallypolarized $W$ bosons in top quark decay as a function of the top and $W$ masses, $m_{t}$ and $M_{W}[10]$

$$
F_{0} \equiv \frac{\Gamma\left(t \rightarrow W_{0}^{+} b\right)}{\Gamma\left(t \rightarrow W_{L}^{+} b\right)+\Gamma\left(t \rightarrow W_{0}^{+} b\right)+\Gamma\left(t \rightarrow W_{R}^{+} b\right)}=\frac{\frac{1}{2}\left(m_{t} / M_{W}\right)^{2}}{1+\frac{1}{2}\left(m_{t} / M_{W}\right)^{2}}
$$

where $\Gamma\left(t \rightarrow W_{R}^{+} b\right)$ is taken to be zero. Because the top quark is so massive, and all of the other quarks are so light, the fraction of longitudinally-polarized $W$ bosons in top decay is relatively large. Using a top mass of $174.3 \pm 5.1 \mathrm{GeV} / \mathrm{c}^{2}$ in the formula

\footnotetext{
${ }^{9} \mathrm{By}$ "polarized" we mean the tendency for the spin of a particle to point in a given direction.
} 
above, the standard model predicts $F_{0}=0.701 \pm 0.012$. As mentioned in the previous section, the fraction of right-handed $W^{+} \mathrm{S}$ in top decay is heavily suppressed due to the $V-A$ structure of the charged-current weak interaction, and in the limit of a massless

$b$ quark is exactly zero. With $m_{t}=175 \mathrm{GeV} / \mathrm{c}^{2}$ and $m_{b}=5 \mathrm{GeV} / \mathrm{c}^{2}$, the fraction of right-handed $W^{+}$bosons in top decay is less than one-tenth of a percent [10].

A direct measurement of the weak-current chirality from the $t W b$ vertex is necessary to validate the $V-A$ form predicted by the standard model [10]. Non-universal weak couplings in top decay will appear as a departure of $F_{0}$ from the standard

value [11], and we can use this quantity as a precision benchmark for probes of anomalous weak couplings. Furthermore, it has been shown that one-loop supersymmetric QCD and electroweak corrections to the total width of $t \rightarrow W b$ could increase the longitudinal fraction as predicted by the standard model by as much as a few percent [12].

\subsection{Techniques for the measurement of $W$ boson polarization}

The $W$ helicity amplitudes are well-known, and the squares of the amplitudes, which govern the angular distributions are written as a function of the quantity [10] $\cos \theta^{*}$ :

$$
\begin{aligned}
\left|\mathcal{M}\left(W_{L}^{+}\right)\right|^{2} & =\left|\mathcal{M}\left(W_{R}^{-}\right)\right|^{2}=\frac{1}{4}\left(1-\cos \theta^{*}\right)^{2} \\
\left|\mathcal{M}\left(W_{0}^{+}\right)\right|^{2} & =\left|\mathcal{M}\left(W_{0}^{-}\right)\right|^{2}=\frac{1}{2}\left(1-\cos ^{2} \theta^{*}\right) \\
\left|\mathcal{M}\left(W_{R}^{+}\right)\right|^{2} & =\left|\mathcal{M}\left(W_{L}^{-}\right)\right|^{2}=\frac{1}{4}\left(1+\cos \theta^{*}\right)^{2}
\end{aligned}
$$

where in the case of leptonic decays of the $W$ boson, $\theta^{*}$ is defined as the angle between the charged-lepton momentum in the $W$ rest-frame and the $W$ momentum in the top- 


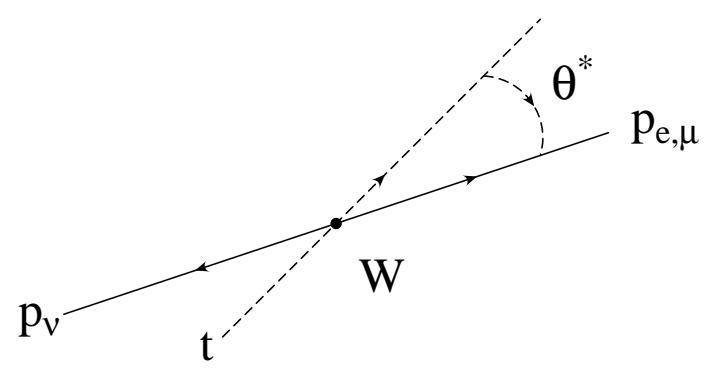

Figure 1.5: The $W$ rest-frame. The angle between the charged-lepton momentum in the $W$ rest-frame and the $W$ momentum in the top rest-frame is defined as the angle $\theta^{*}$.

quark rest-frame (Figure 1.5). Note that the angular distributions for the $W_{L}^{+}$and $W_{R}^{-}$helicity amplitudes are identical, as are the $W_{R}^{+}$and $W_{L}^{-}$amplitudes. Throughout the remainder of this thesis, as a convenient simplification, I will use "left-handed" to denote the $W_{L}^{+}$and $W_{R}^{-}$transverse states, "right-handed" to denote the $W_{R}^{+}$and $W_{L}^{-}$states, and "longitudinal" to signify both $W_{0}^{+}$and $W_{0}^{-}$.

From the amplitudes in Eqns 1.9-1.11, one can conclude that events with charged leptons from longitudinal $W$ decay have an angular distribution which peaks at $\theta^{*}=$ $\pi / 2$; charged leptons from the decay of a transverse bosons are most likely to have $\theta^{*}=0$ (right-handed) or $\theta^{*}=\pi$ (left-handed). The resulting $\cos \theta^{*}$ distributions are very distinct for each $W$ helicity state, and are shown in Figure 1.6. If one is able to reconstruct the $\cos \theta^{*}$ distribution from top quark decays observed in collider data, these unique shapes can be used for a measurement of $W$ boson polarization and a comparison with theory could be made.

We can express the $\cos \theta^{*}$ quantity explicitly by taking the dot-product of the charged-lepton momentum vector in the $W$ rest-frame, $\left(\vec{p}_{l}\right)_{W}$, and the $W$ boson momentum in the top-quark rest-frame, $\left(\vec{p}_{W}\right)_{t}$

$$
\cos \theta^{*}=\frac{\left(\vec{p}_{l}\right)_{W} \cdot\left(\vec{p}_{W}\right)_{t}}{\left|\left(\vec{p}_{l}\right)_{W}\right|\left|\left(\vec{p}_{W}\right)_{t}\right|} .
$$



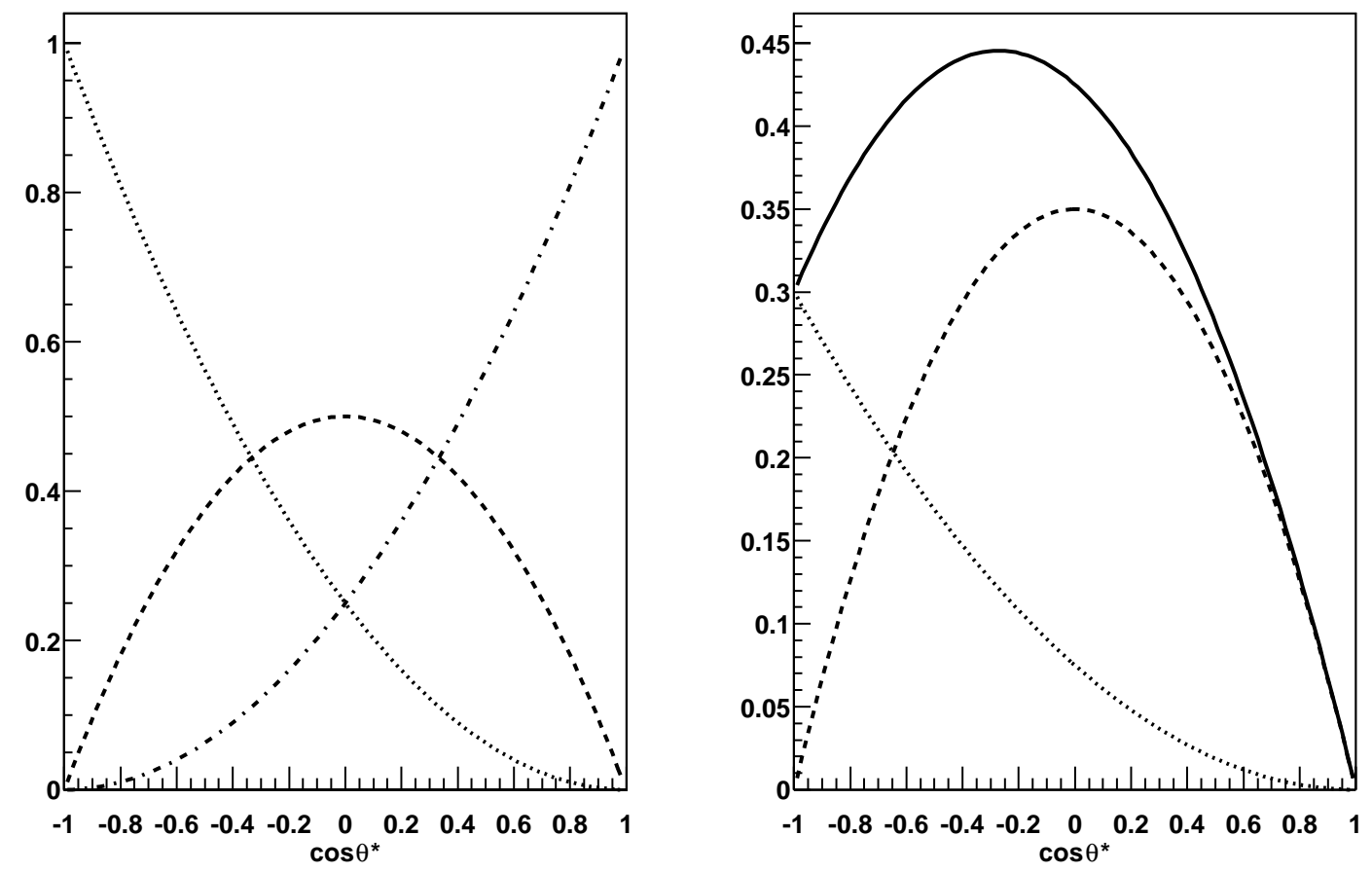

Figure 1.6: Left: The true $\cos \theta^{*}$ distribution (Equation 1.12) for left-handed $W$ bosons (dotted line), longitudinal $W \mathrm{~s}$ (dashed line), and right-handed $W \mathrm{~s}$ (dashdotted line). Right: The solid line indicates the standard model $\cos \theta^{*}$ distribution which is roughly a $70 \% / 30 \%$ split between longitudinal (dashed) and left-handed (dotted) helicity $W \mathrm{~s}$. The contribution from right-handed $W \mathrm{~s}$ is negligible in the standard model $(<0.1 \%[10])$. 
However a direct measurement of $W$ boson polarization through an analysis of the $\cos \theta^{*}$ distribution is difficult, as it relies on our ability to completely reconstruct the $t \bar{t}$ event, which means that we must have enough kinematic information to extract the parent particle momentum vectors. In the case where both $W \mathrm{~s}$ from a $t \bar{t}$ pair decayed leptonically, we are unable to do this unambiguously since we lack sufficient information about the momentum vectors of the two neutrinos. It is possible to construct events where only one of the $W$ s decayed leptonically and the other hadronically, dispite the fact that we still lack momentum information for one neutrino in the event, but it is difficult to precicely match each hadronic jet to the quark which produced them. This potential for jet mismatching gives rise to systematic uncertainties. Although Equations 1.9-1.11 are valid for all hadronic decays of the $t \bar{t}$ pair, to take full advantage of these amplitudes we need to know which daughter of the $W$ is the quark and which is the anti-quark. From a detector standpoint we have no reliable way of identifying the quark or anti-quark from such a decay; we would need to reconstruct and determine the charges of the daughter quarks. Thankfully, in $t \bar{t}$ events where one or both of the $W \mathrm{~s}$ decays leptonically, measuring the charge of the resulting leptons is not difficult. To determine which daughter is the fermion and which is the anti-fermion we simply need to track the direction of the charged lepton as it curves in response to a magnetic field. The most accurate measurement of $W$ polarization will be extracted from $t \bar{t}$ events where at least one of the $W$ s decays leptonically; the analysis described in this thesis was conducted on such a dataset. It is worth noting that a measurement in the leptonic channel $(t \rightarrow W b \rightarrow l \nu b)$ is not free from difficulties either. The $\tau$ lepton, with an extremely short lifetime of $\sim 10^{-13}$ $\mathrm{s}$, will only travel a few hundred $\mu \mathrm{m}$ before decaying, most often hadronically. Due to the complications and systematic uncertainties associated with the $\tau$ channel, it is excluded from this analysis.

The data sets seriously considered for the measurement of $W$ boson polarization 
carried out in this thesis consist of $t \bar{t}$ pairs which decay into $W^{+} b$ and $W^{-} \bar{b}$, where one or both of the $W$ s decayed leptonically into an electron or a muon. The "dilepton" data sample contains $t \bar{t}$ events where both $W$ s have decayed leptonically. When only one $W$ decays leptonically, and the other hadronically in to a $q \bar{q}^{\prime}$ pair, that event is included in the "lepton-plus-jets" data set. Using these data sets there are at least two methods one may use to extract the degree of $W$ boson polarization in top quark decays. One method uses the lepton angular distriubution, $\cos \theta^{*}$, the other method examines the charged lepton $p_{T}$ distribution ${ }^{10}$; both methods were investigated at CDF during Run I $[13,14,15,16]$ of the Tevatron. Both methods are discussed in further detail below.

\subsubsection{The $\cos \theta^{*}$ Method}

The analysis described in this thesis extracts the quantity $\cos \theta^{*}$ from the $p \bar{p}$ collision data, and compares the results obtained with our expectations. These expectations are quantified by a series of templates created from events generated through the use of event simulations. The details regarding these simulations, as well as the method used to fit templates to detector data, are discussed in Chapters 4 and 5. Here I only give a brief overview of what is needed to extract $\cos \theta^{*}$ from the data.

Since we cannot reliably reconstruct either the $W$ or the top-quark rest-frames, it is extremely difficult to measure $\cos \theta^{*}$ explicitly (Equation 1.12). To circumvent this difficulty, the analysis described in this thesis exploits an approximation for $\cos \theta^{*}$ given as [10]:

$$
\cos \theta^{*} \simeq \frac{2 m_{l b}^{2}}{m_{t}^{2}-M_{W}^{2}}-1
$$

where $m_{l b}$ is the invariant mass of the charged-lepton from the $W$ boson and the $b$ quark from the decay $t \rightarrow W b \rightarrow l \nu b, m_{t}$ and $M_{W}$ are the event-by-event top quark

\footnotetext{
${ }^{10} p_{T}$ is used to denote the projection of the momentum of a particle in the plane perpendicular to the direction of the $p \bar{p}$ beam. $p_{T}$ is used with great frequency in particle detector measurements because the momentum of the initial partons in the beam is unknown. In coordinates used by CDF, $p_{T}^{2}=p_{x}^{2}+p_{y}^{2}$.
} 
and $W$ boson masses, respectively.

The same difficulties that prohibit us from reliably reconstructing the top and $W$ rest-frames prevent accurate measurements of the event-by-event top and $W$ masses. Because the use of inaccurate event-by-event masses would unnecessarily smear the $\cos \theta^{*}$ distribution obtained from the $m_{l b}$ approximation, reducing the precision of the measurement, this analysis uses the the world-averaged masses of $m_{t}=174.3$ and $M_{W}=80.4$ in Equation 1.13. Strictly using the world-averaged masses in the approximation for every event affects the distribution by introducing values of $\cos \theta^{*}>$ 1 when $m_{l b}^{2}>m_{t}^{2}-M_{W}^{2}$ (for a comparison of these distributions see Figures 1.7 and 1.8; note the small number of events with $\cos \theta^{*}>1$ in Figure 1.8). The occurrence of values $>1$ does not adversely affect the measurement, and is simply a feature of reducing Equation 1.13 to $c \cdot m_{l b}^{2}-1$, where $c$ is a constant. Note that because $m_{l b}$ is squared in this approximation, it is not possible to obtain $\cos \theta^{*}$ values $<-1$.

In the decay of $t \bar{t}$ events there are two $b$ quarks that could be selected to calculate the invariant mass $m_{l b}$. In order to gauge the effect on the measurement of using the four-momenta from the incorrect $b$ quark (i.e., that from the decay $t \rightarrow q \bar{q}^{\prime} b$ which from now on will be referred to as the "hadronic- $b$ ", instead of $t \rightarrow l \nu b$ which we will refer to as the "leptonic- $b$ ") in the calculation of $m_{l b}$, a series of "pseudoexperiments" were conducted and the difference in the precision of the measurement was noted. Pseudo-experiments are sets of measurements carried out on strictly simulated data comprised of an expected number of signal and background events. The measurements made on simulated data during these pseudo-experiments are done in a manner identical to those performed on the actual detector data (Chapter 4 presents a more in-depth discussion of this procedure). Figures 1.8 and 1.9 show the $\cos \theta^{*}$ shapes obtained from simulated data when using four-momenta information for the correct and incorrect $b$, respectively. The shapes from these figures were used as templates to fit simulated data during pseudo-experiments; multiple sets of experiments 
were conducted where each set has a unique true longitudinal fraction. This true fraction was varied from set-to-set between 0.5 and 0.7 in steps of 0.5 . The results from these pseudo-experiments are plotted in Figure 1.10, a large difference in preci-

sion is apparent. The results of these pseudo-experiments indicate the importance of selecting the correct $b$ quark when calculating $m_{l b}$.

For the analysis carried out in this thesis, an event-by-event kinematic fitter is used to reconstruct the events and assign a jet to the parton-level $b$ quark in leptonplus-jets events. Due to the requirements of this fitter, the difficulty in choosing the correct particle four-vectors in dilepton events and twice the neutrino ambiguity in such events, using the dataset comprised of dilepton events is precluded. The kinematic fitter used by this analysis is discussed further in Chapter 4.

An analysis using the $m_{l b}^{2}$ distribution alone to measure the fraction of $V+A$ was done at CDF during the first run of the Tevatron $[15,16]$.

\subsubsection{The Lepton $p_{T}$ Method}

It is worth noting that as an alternative to reconstructing the $\cos \theta^{*}$ distribution, one can measure $W$ boson polarization using the charged-lepton $p_{T}$ distribution. Lepton $p_{T}$ is the most precisely measured quantity in a $t \bar{t}$ event. Close examination of the helicity amplitudes in Equations 1.9-1.11 reveals that for the case of longitudinal $W$ decay, the charged lepton has the highest probability of decaying in a direction perpendicular to the $W$ momentum in the top rest-frame; likewise, a charged lepton from a transverse $W$ has the highest likelihood of decaying in a direction parallel the $W$ momentum as viewed from the top rest-frame. Such a bias in the charged lepton momentum distributions from the decay of transverse and longitudinal $W \mathrm{~s}$ is carried over into the lab-frame, where we can observe three very distinct $p_{T}$ distributions (see Figure 1.11).

To conduct such a measurement one compares the lepton $p_{T}$ distributions mea- 
sured from detector data with those generated using simulated $t \bar{t}$ events. This technique has been used successfully to measure the fraction of $W$ boson polarization in top quark decay at CDF during both Run I [13, 14] and Run II [17]. Although the $\cos \theta^{*}$ method utilizes the lepton angular distribution, thus making it a very powerful analysis strategy by providing more information per event, the lepton $p_{T}$ method has a greater statistical advantage since it can use both the lepton-plus-jets and dilepton samples. It is also worth noting that much of the discriminating power of the lepton $p_{T}$ technique lies at very low $p_{T}$, however $t \bar{t}$ event selection requires $p_{T} \geq 20$ (event selection is detailed in Chapter 3). 

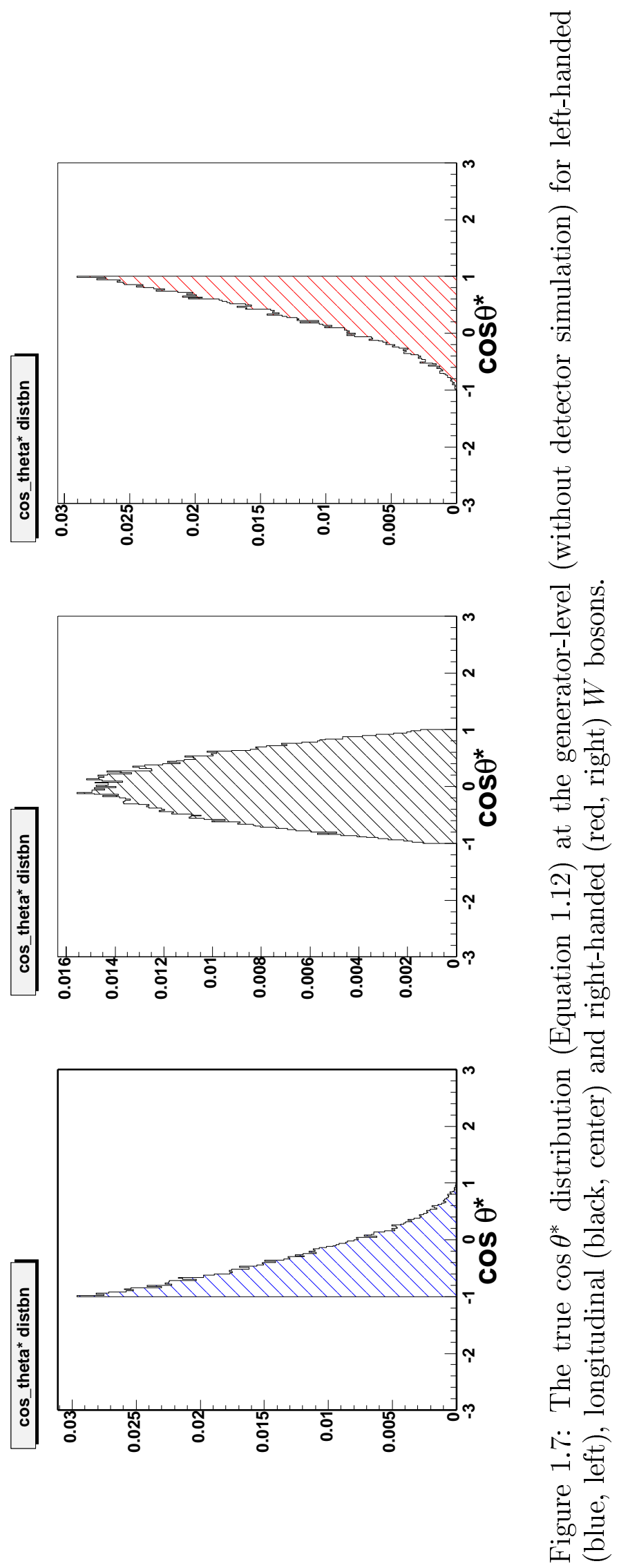


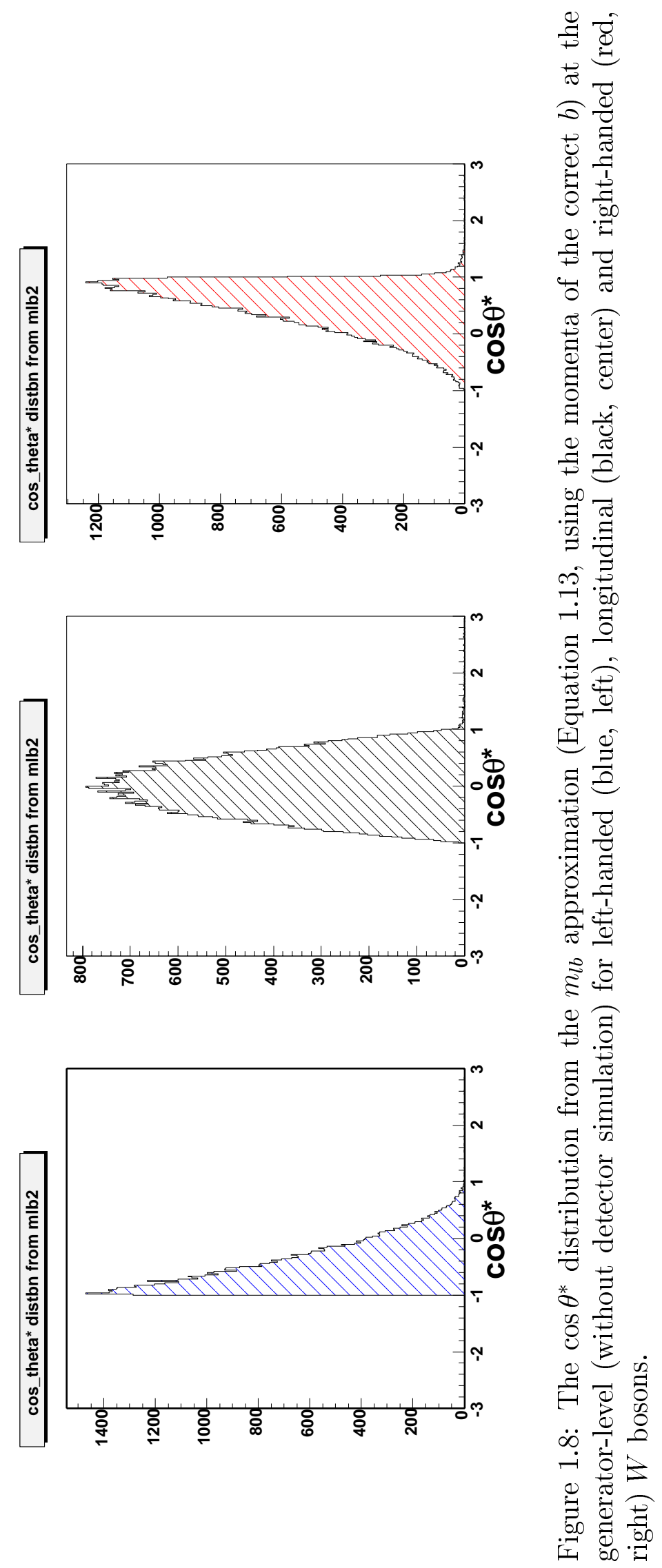




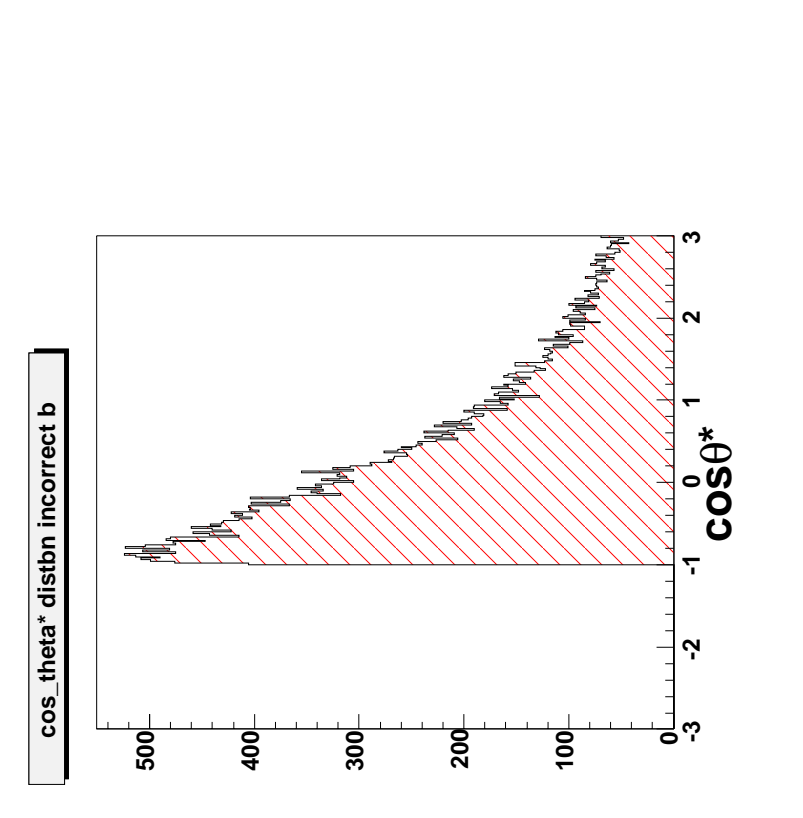

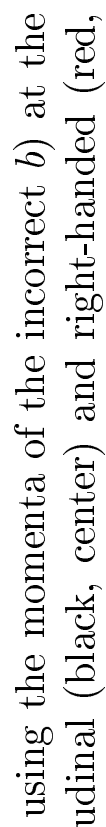

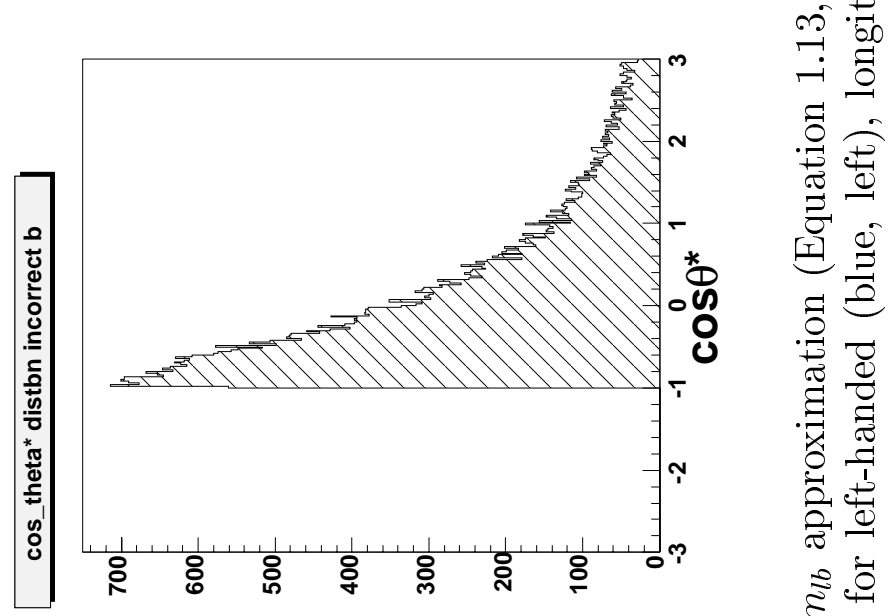

है

옳

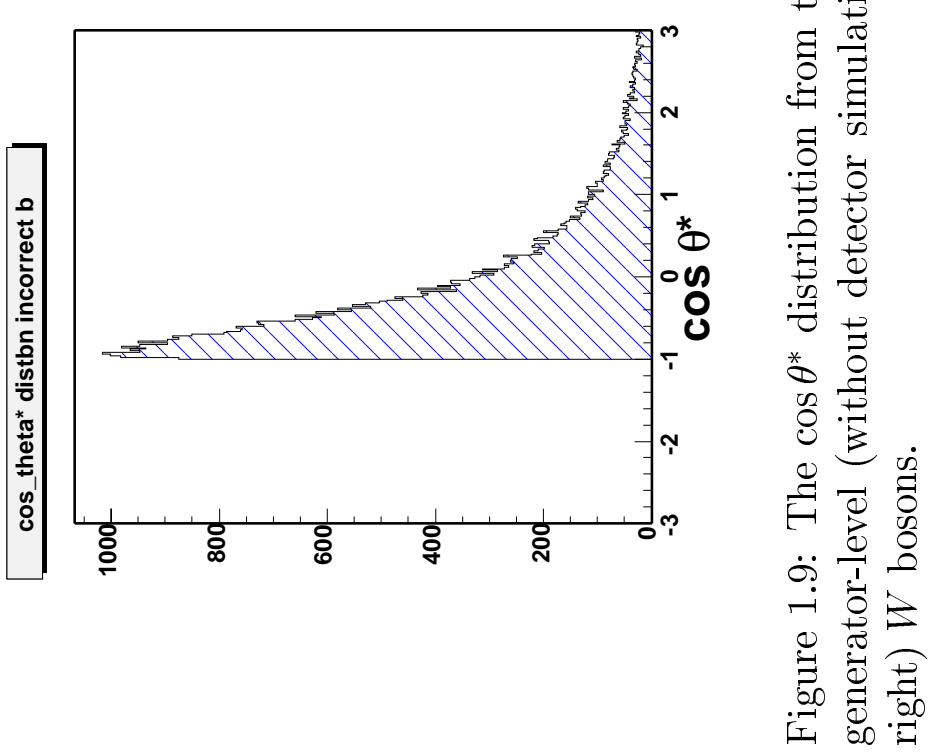




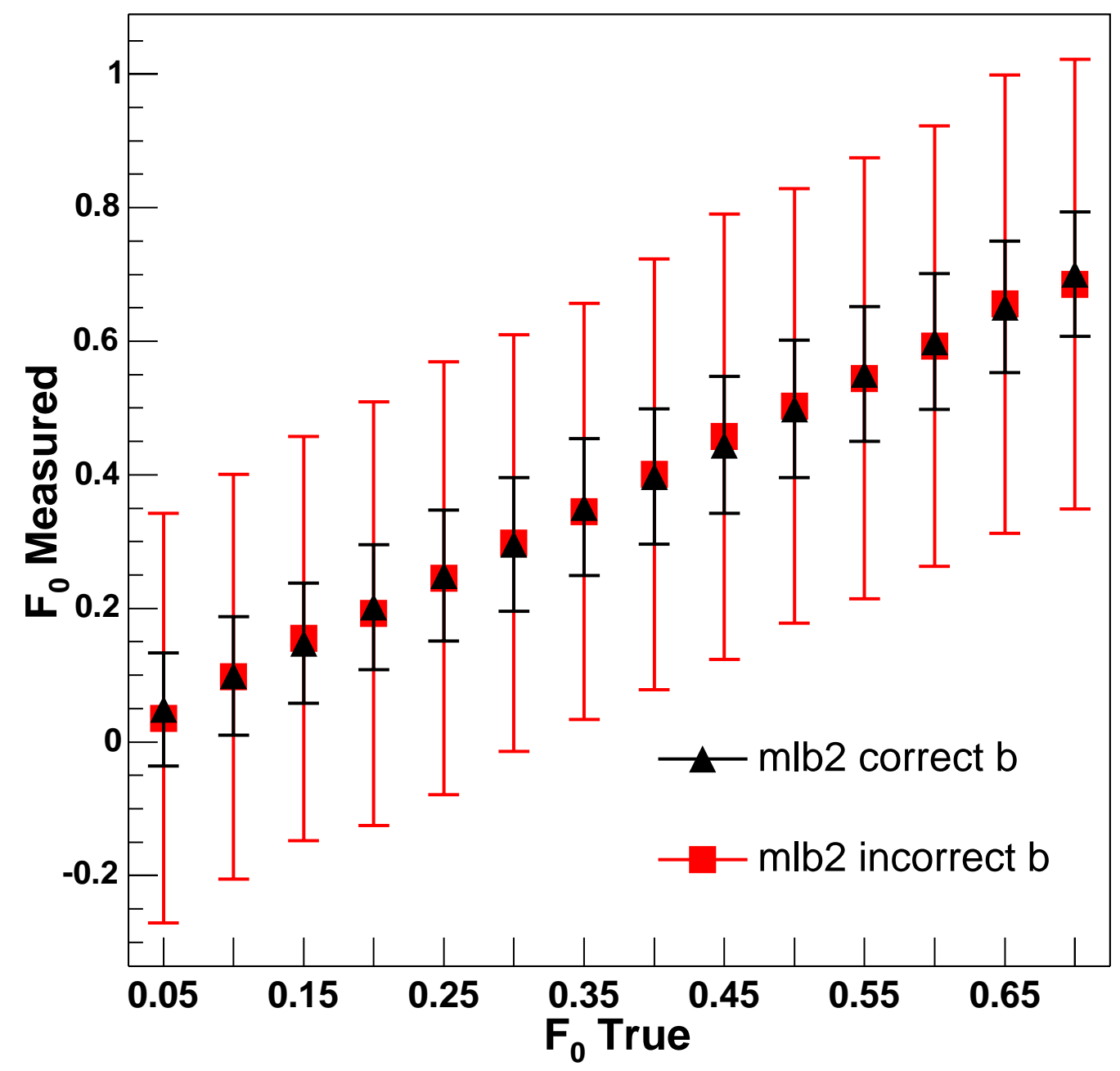

Figure 1.10: The expected precision of measurements of the longitudinal fraction, $F_{0}$, when running pseudo-experiments at the generator level (without detector simulation) fitted to templates created using the $m_{l b}$ approximation to obtain the $\cos \theta^{*}$ distributions (Equation 1.13). The results shown are for pseudo-experiments with 14 different values of $F_{0}$ between 0.05 and 0.70 , in steps of 0.05 . It is clear from this plot that using the correct $b$ in the $m_{l b}$ approximation yields higher precision (black triangle) than for templates where $\cos \theta^{*}$ was calculated using the incorrect $b$ (red square). 


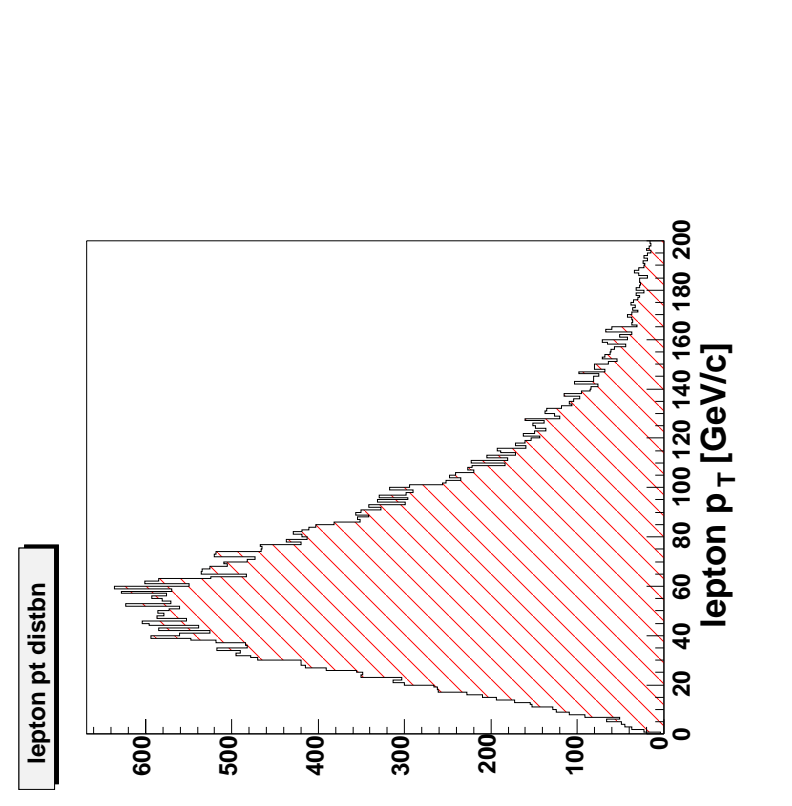

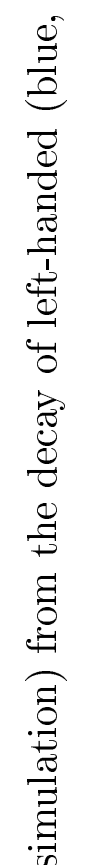

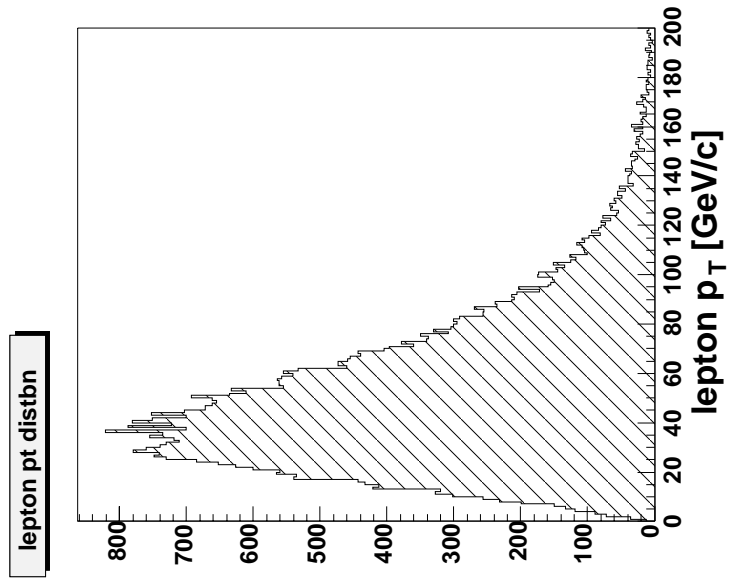

o

苍

के

3.

要

声

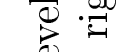

웡

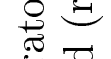

离

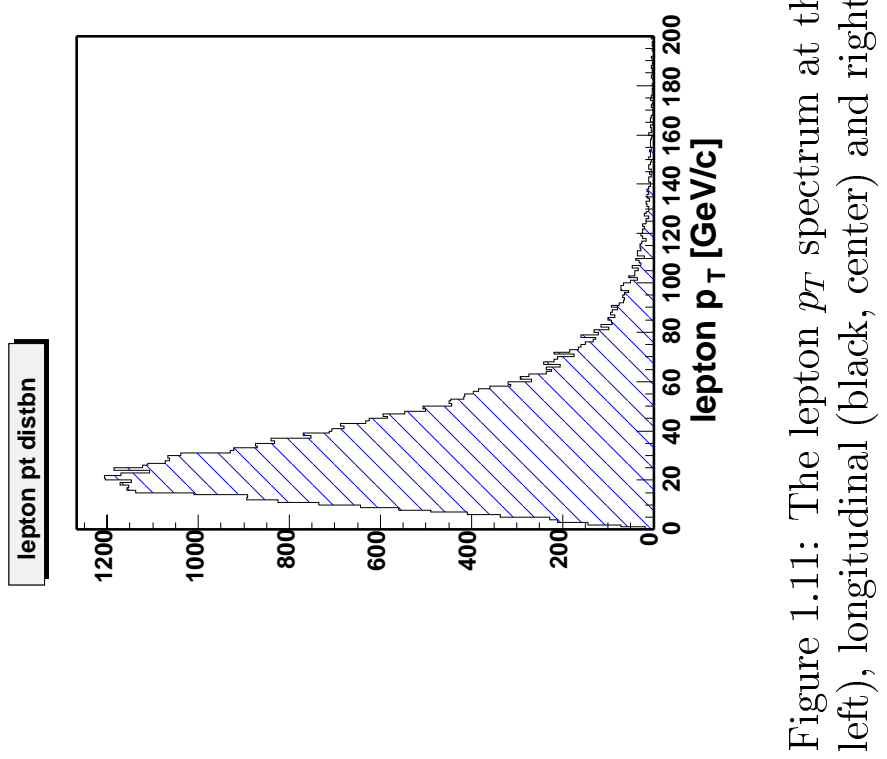




\section{Chapter 2}

\section{The Fermilab Tevatron and the CDF II Detector}

The Tevatron is a superconducting proton-antiproton $(p \bar{p})$ accelerator located at the Fermi National Accelerator Laboratory (FNAL) in Batavia, Illinois. FNAL is home to a complex of several accelerators and storage rings which supply protons and antiprotons to the two Tevatron collider experiments, as well as delivering particle beams to fixed-target experiments.

The data used for the analysis described in this thesis were collected with the Collider Detector at Fermilab (CDF), one of the two Tevatron collider experiments, between March 2002 and September 2003-the beginning of what is known as Run IIa. In this chapter I describe the Tevatron accelerator and the CDF II experimental apparatus.

\subsection{The Tevatron}

The protons for collisions and the production of antiprotons originally come from hydrogen gas $\left(\mathrm{H}_{2}\right)$ which is negatively ionized by a Haefely Cockcroft-Walton preaccelerator $[18,19,20]$ known as the magnetron. A dome inside the pre-accelerator houses the ions and is charged to a potential of $-750 \mathrm{keV}$; the $\mathrm{H}^{-}$ions accelerate to energies of $750 \mathrm{keV}$ through a column from the charged dome to the grounded 


\begin{tabular}{|c|c|}
\hline \hline Machine & Energy \\
\hline Cockroft-Walton & $750 \mathrm{keV}$ \\
\hline Linac & $400 \mathrm{MeV}$ \\
\hline Booster & $8 \mathrm{GeV}$ \\
\hline $\begin{array}{c}\text { Main Injector } \\
(\bar{p} \text { production) }\end{array}$ & $120 \mathrm{GeV}$ \\
\hline $\begin{array}{c}\text { Main Injector } \\
\text { (Tevatron injection) }\end{array}$ & $150 \mathrm{GeV}$ \\
\hline Debuncher/Accumulator/Recycler & $8 \mathrm{GeV}$ \\
\hline Tevatron & $980 \mathrm{GeV}$ \\
\hline \hline
\end{tabular}

Table 2.1: Machines and corresponding energies at the Fermilab accelerator complex [25].

wall prior to being injected into a $150 \mathrm{~m}$ long linear accelerator, known as the Linac. The Linac $[21,22,23,24]$ uses a chain of radiofrequency cavity structures to further accelerate the negatively charged ions from an energy of $750 \mathrm{keV}$ up to $400 \mathrm{MeV}$. Electrons are then stripped off of the hydrogen ions by passing this $400 \mathrm{MeV}$ beam through a thin carbon film [25]. After being stripped of their electrons, the hydrogen nuclei (protons) are passed on to the Booster ring for the next stage of acceleration. The Booster [26] is the first circular accelerator, or synchrotron ${ }^{1}$, in the accelerator chain and is used to accelerate protons up to energies of $8 \mathrm{GeV}$ and gather them into bunches. The Main Injector [28], a larger synchrotron with a circumference several times that of the Booster, is the next machine in the accelerator chain. The Main Injector accelerates these proton bunches to energies up to $150 \mathrm{GeV}$ and coalesces them into a single bunch before injecting them into the Tevatron ring for the final stage of acceleration. A portion of these protons are accelerated by the Main Injector to 120 $\mathrm{GeV}$ and used for the production of antiprotons. A summary of machine energies are provided in Table 2.1 and a schematic of the Fermilab accelerator complex is shown in Figure 2.1.

\footnotetext{
${ }^{1} \mathrm{~A}$ synchrotron is more complex than a simple cyclotron in the sense that the $\mathrm{B}$ field of the former must be increased synchronously with the particle energy [27].
} 
To produce antiprotons [29], $120 \mathrm{GeV}$ protons from the Main Injector collide with a nickel-copper target [30]. This collision produces a multitude of secondary particles, some of which are antiprotons. An axis-symmetric electromagnetic lithium lens collects the divergent secondary antiprotons and focuses the resulting cone of secondary particles into a parallel beam. The lens consists of a $15 \mathrm{~cm}$ lithium conductor contained inside of a $1 \mathrm{~mm}$ titanium tube surrounded by cooling water and is capped off with beryllium end-windows. Lithium is used as the electrical conductor to minimize absorption and scattering as the secondary beam passes through the lens. The lithium lens has a short focal length and is capable of producing a magnetic field gradient of $750 \mathrm{~T} \cdot \mathrm{m}^{-1}$ [31]. The use of a lithium lens instead of a magnetic horn or conventional quadrupoles increases antiproton yield by at least a factor of four [31]. A pulsed dipole magnet is used to selectively choose the charge and momentum of particles collected from the spray; in this manner, $8 \mathrm{GeV}$ antiprotons are harvested from the collision with the target. ${ }^{2}$ The momentum spread of the $8 \mathrm{GeV}$ beam from the target is initially quite wide and will lead to a large emittance of the antiproton beam if not reduced. This momentum spread is diminished inside the Debuncher [32] through the application of betatron (transverse) stochastic cooling [33, 34] and momentum (longitudinal) cooling. After cooling, the antiprotons are extracted from the Debuncher and injected into the Accumulator [35] for temporary storage. When enough antiprotons have been accumulated a transfer into the Main Injector and then ultimately the Tevatron will be initiated.

36 bunches of counter-rotating protons and antiprotons are distributed around the $6 \mathrm{~km}$ circumference of the Tevatron ring, for the final stage of acceleration. Inside the Tevatron each beam attains an energy of $980 \mathrm{GeV}$, which gives center-of-mass energies, ${ }^{3} \sqrt{s}$, for collisions of $1.96 \mathrm{TeV} .36$ bunches of protons collide with 36 bunches

\footnotetext{
${ }^{2}$ This process is extremely inefficient. For every $10^{5}$ protons which strike the target, it is typical for only one or two antiprotons to be captured and stored.

${ }^{3}$ The total energy of colliding particles is often referred to in terms of the Mandelstam variable $s$, which is invariant under Lorentz transformations.
} 


\section{FERMILAB'S ACCELERATOR CHAIN}

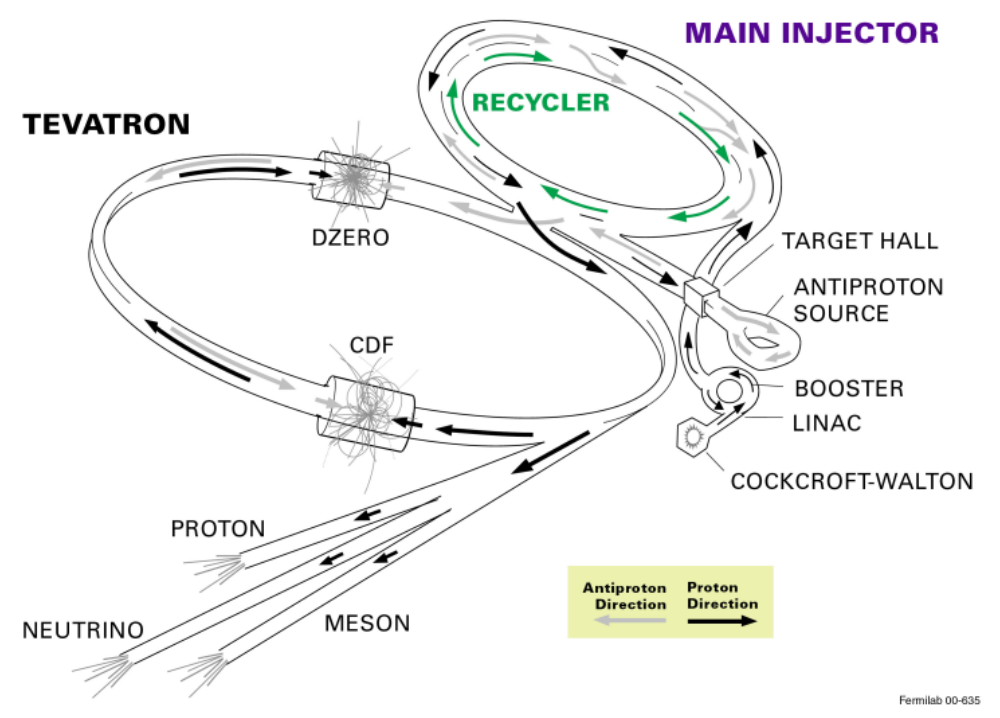

Figure 2.1: The Fermilab Accelerator chain [36].

of antiprotons; one bunch of protons and antiprotons collide every $396 \mathrm{~ns}$.

The period of time when colliding beams are present in the Tevatron is referred to as a "store". During a typical Run IIa store, there are 36 bunches of protons, each containing $24 \times 10^{10}$ protons and 36 bunches of antiprotons, each containing $3 \times 10^{10}$ antiprotons. The counter-rotating proton and antiproton bunches travel around the Tevatron ring with a velocity quite close to the speed of light, passing each other undisturbed by traveling in electrostatically-separated helical orbits, and focused to collide together only at the desired interaction points B0 and D0 where the CDF and D0 experiments reside.

The intensity of the beams is known as "luminosity" and can be calculated using the formula ${ }^{4}$

$$
\mathcal{L}=n f \frac{N_{p} N_{\bar{p}}}{4 \pi \sigma_{x} \sigma_{y}},
$$

where $n$ denotes the number of bunches, $f=50 \mathrm{kHz}$ is the revolution frequency for

\footnotetext{
${ }^{4}$ This is a simplified formula for calculating the luminosity and neglects the "hourglass-factor" and the $\beta$-factor from convoluting two Gaussian bunches in $z$.
} 
one bunch traveling near the speed of light around the circumference of the Tevatron ring, $N_{p}$ and $N_{\bar{p}}$ are the number of protons and antiprotons per bunch, respectively. $\sigma_{x}$ and $\sigma_{y}$ are the Gaussian beam profiles in the transverse plane, averaged over $z$; the beam cross-section is roughly circular with a radius of $\sim 35 \mu \mathrm{m}$. Using these values in Equation 2.1 yields a luminosity of $\sim 8.0 \times 10^{31} \mathrm{~cm}^{-2} \mathrm{~s}^{-1}$. At the time that this analysis was done, the Tevatron was routinely providing stores with this initial luminosity. As collisions with a particular store progress, the number of protons and antiprotons in each bunch decrease as a function of time. This decrease is due to losses resulting from proton-antiproton annihilations, as well as losses due to orbit variations.

It is through the high-energy interactions which result from these proton-antiproton collisions that $t \bar{t}$ pairs used in the analysis described in this thesis are produced. The Tevatron, with a center-of-mass energy of $1.96 \mathrm{TeV}$ for collisions, is currently the highest-energy particle accelerator on Earth and the only facility producing top quarks. The data used in this analysis were collected with the Collider Detector at Fermilab between March 2002 and September 2003, and corresponds to $\sim 162 \mathrm{pb}^{-1}$ of luminosity ${ }^{5}$ integrated over this time period.

\subsection{The CDF II Detector}

The particles that are created as a result of proton-antiproton collisions are detected through their interaction with matter. For charged particles this interaction is predominantly through hadronic interactions which ionize and excite the detector medium. The ideal particle detector would provide coverage for the full $4 \pi$ solid angle; provide precise measurements of energy and momentum; detect, track and

\footnotetext{
${ }^{5}$ A "barn", b, is a unit of effective nuclear cross-secion, $10^{-24} \mathrm{~cm}^{2}$ per nucleus. A value of $10^{-24}$ $\mathrm{cm}^{2}$ was commonly used as a unit for nuclear cross-sections in work for the Manhattan project, and was aptly named by M. G. Holloway and C. P. Baker in December 1942 because "a cross section of $10^{-24} \mathrm{~cm}^{2}$ for nuclear processes was really as big as a barn" [37].
} 
identify all particles in terms of charge and mass; offer fast response with absolutely no "dead-time" due to detector readout.

CDF is a general purpose solenoidal detector [38] built around one of the $p \bar{p}$ collision points (B0) of the Tevatron accelerator. CDF is actually a collection of several sub-detectors designed to work together to identify a variety of particles produced in $p \bar{p}$ collisions at the Tevatron. The end result of this arrangement is a detector with precision charged particle tracking, fast projective calorimetry and fine muon resolution. The CDF experiment first saw proton-antiproton collisions in 1985 and has evolved over time to become increasingly more sensitive. CDF collected about $110 \mathrm{pb}^{-1}$ at $\sqrt{s}=1.8 \mathrm{TeV}$ during Run I of the Tevatron (1992-1996). Over a nearly five year hiatus, CDF underwent massive upgrades to cope with increased design luminosity $\left(2 \times 10^{32} \mathrm{~cm}^{-2} \mathrm{~s}^{-1}\right)$, higher center-of-mass energy $(\sqrt{s}=1.96 \mathrm{TeV})$, and six-times more frequent bunch crossings (one collision every $396 \mathrm{~ns}$ ).

The CDF II detector (Figure 2.2) is designed around a central superconducting solenoid which provides a constant $1.4 \mathrm{~T}$ magnetic field, and hence inherits a cylindrical geometry. The magnetic field of the solenoid is parallel to the colliding beams, thus avoiding both forces on the beam particles and the production of synchrotron radiation. The detector is azimuthally and forward-backward symmetric with the entire apparatus centered about the interaction point, B0, where the $p$ and $\bar{p}$ beams are focused to collide using quadrapole magnets; hence collisions occur in the center of the detector. A beampipe, with a radius of $\sim 1 \mathrm{~cm}$ at the $\mathrm{B} 0$ interaction point, runs through the center of the CDF detector along the $z$-axis and houses the circulating particles. The coordinate system of $\mathrm{CDF}$ is $(\eta, \phi, z)$, where the longitudinal axis ( $z$-axis) of the detector runs parallel to the proton direction. The azimuthal angle is $\phi$, and $\eta$ is the "pseudorapidity" $"$ variable given as $\eta=-\ln \left(\tan \left(\frac{\theta}{2}\right)\right)$, where the polar

\footnotetext{
${ }^{6}$ When particles are relativistic $(p>>m), \eta$ is a good approximation of the true rapidity. The rapidity, $y$, is defined as $y \equiv \tanh ^{-1} p_{z} / E$. The pseudorapidity of a particle can easily be measured even though the mass and momentum are unknown.
} 


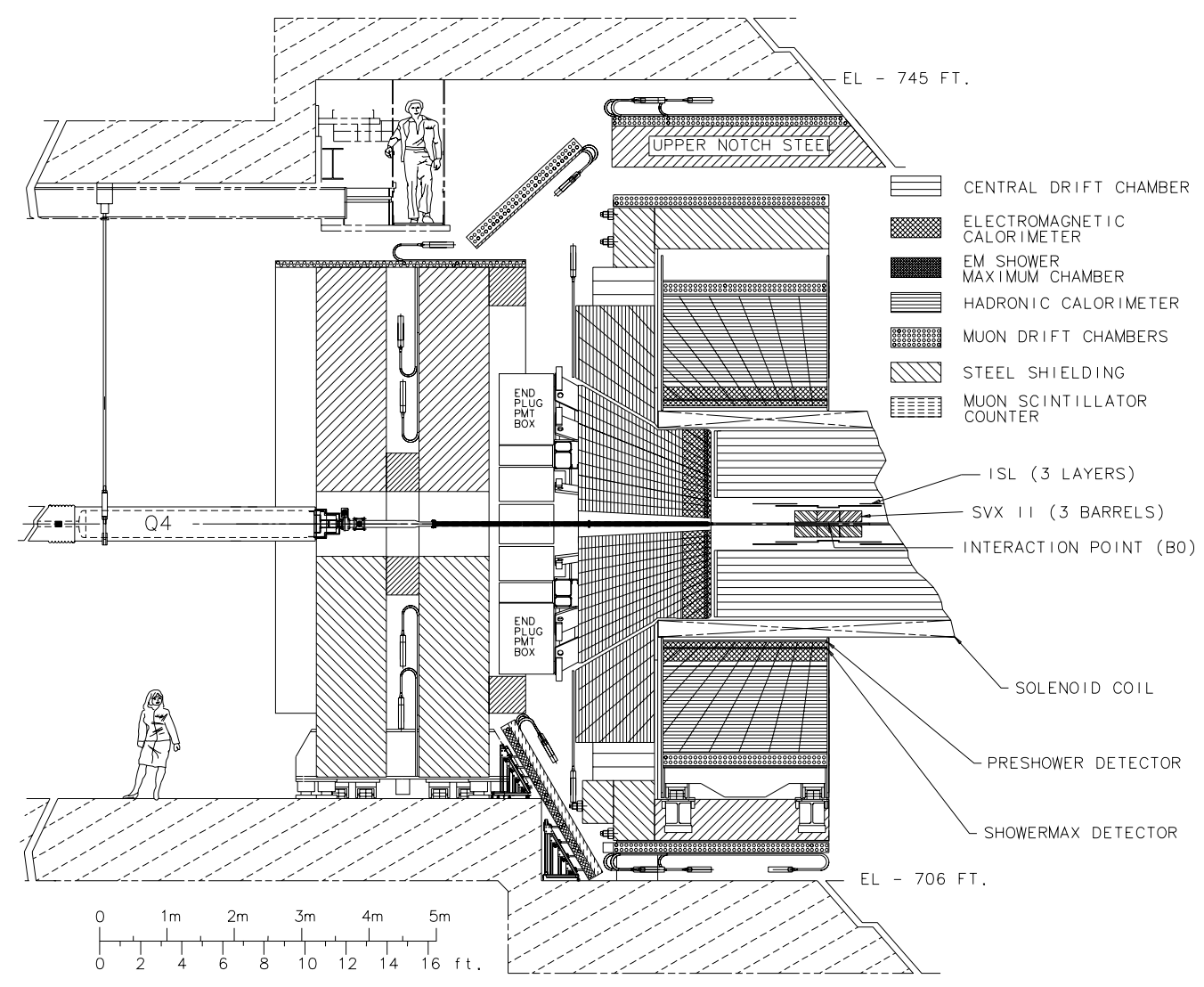

Figure 2.2: Elevation view of one half of the CDF II detector [38]. 
angle $\theta$ is measured from the proton direction (pseudorapidity transforms linearly under Lorentz boosts in the $z$-direction and $\Delta \eta$ is an invariant [2]). The range of $|\eta|$ is from 0 at the perpendicular to the beampipe to $\sim 3.5$ at the most forward region of the detector (see Figure 2.3). The central-most region of CDF falls in the range of $0 \leq|\eta| \leq 1.0$ while the forward detectors are located in the range $1.0<|\eta| \leq 3.0$. As mentioned earlier, CDF is a collection of detectors that work in concert to identify and then measure the properties of particles produced in $p \bar{p}$ collisions. Detector subsystems are placed radially at various distances from the interaction point; this placement is strategic in the sense that tracking information is recorded closest to the interaction point, muon detection is done furthest from the interaction point, and the measurement of particle energy is performed between these two regions. In general, these subsystems can be divided into several categories: particle tracking systems, calorimetry, muon detectors and triggering systems. Due to the large number of subsystems that make up CDF, the remainder of this chapter will only focus on those components of the detector that were relevant to the analysis described in this thesis. A detailed description of the entire CDF II apparatus can be found in [38].

\subsubsection{Tracking Systems}

The paths of charged particles through CDF are recorded through the use of tracking detectors. Tracking information is crucial for the measurement of particle momenta and identification of particle charge. The Lorentz force law, $\vec{F}=q \vec{v} \times \vec{B}$, is exploited by particle physicists who desire to know both the sign of the charge (assuming $q=0, \pm 1)$ and momentum of particles passing through the detector. It is for this reason that the tracking systems of CDF are contained inside a solenoid [39] with a uniform 1.4 T magnetic field which runs parallel to the beam axis (-z-direction). Due to the magnetic field created by the solenoid, charged particles traveling through the central volume of the detector follow a helical path. The radius of this helix is 
related the the transverse momentum of the particle:

$$
p_{T}=\frac{0.3 B}{2 C}
$$

expressed in units of $\mathrm{GeV} / \mathrm{c}$, where $B$ is the magnitude of the magnetic field in Tesla and the half-curvature, $C$ is in $\mathrm{m}^{-1}$ and is related to the radius of the helix by $C=1 / 2 R$. The helix of a charged track can be described by the following five parameters:

- $\cot \theta$, the co-tangent of the polar angle $\theta$ of the particle at the point of closest approach to the origin.

- $C$, the half-curvature. This variable has the same sign as the charge of the particle.

- $z_{0}$, The $z$ position of the particle at the point of closest approach to the origin.

- $d_{0}$, the impact parameter. The distance between the helix and the origin at the point of closest approach to the origin, in the transverse plane.

- $\phi_{0}$, the azimuthal angle of the particle trajectory. The direction of a track at the point of closest approach to the origin.

Concentrically arranged inside the solenoid magnet at various radii are CDFs tracking systems. The inner-most system is a silicon microstrip detector [40] which extends from a radius of $1.5 \mathrm{~cm}$ to $10.7 \mathrm{~cm}$ from the center of CDF and is known as the Silicon VerteX detector (SVX). Radially positioned between 20 and $28 \mathrm{~cm}$ is the Intermediate Silicon Layer (ISL). Finally, the Central Outer Tracker (COT) is an open-cell wire drift chamber [41] which covers between 22 to $132 \mathrm{~cm}$ in radius (see Figure 2.3).

The SVX provides precision stand-alone tracking information at the smallest possible radii $(i . e .$, closest to the interaction point where the proton and antiproton beams 


\section{CDF Tracking Volume}

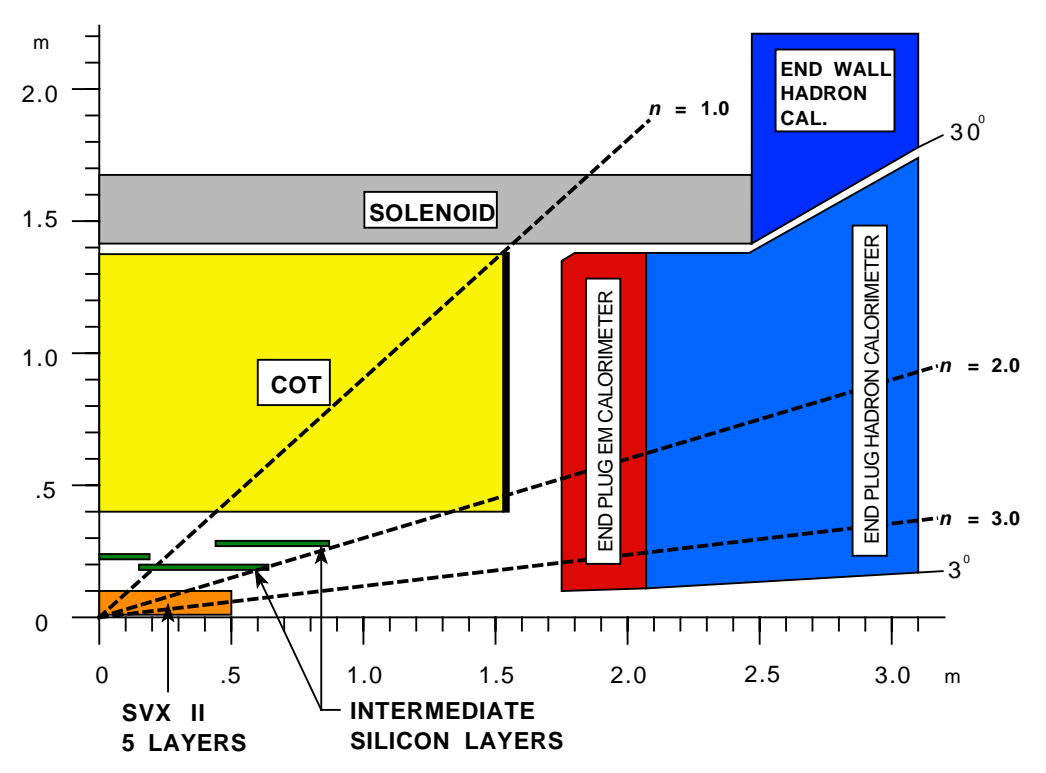

Figure 2.3: A cross-sectional view of the most central regions of the CDF II detector with $\eta$ superimposed [38].

collide) through the use of $\sim 11 \mathrm{~m}^{2}$ of silicon microstrip detectors arranged in a barrel geometry consisting of multiple layers with a readout pitch of $\sim 60 \mu \mathrm{m}$ in $r-\phi$ and $r-z$. The microstrips are semiconductor detectors created from a $\sim 300 \mu$ m thick bulk layer of lightly-doped $n$-type silicon sandwiched between a strongly-doped $n$ electrode and a lightly-doped $p$ region. A positive voltage is applied to the $n$ electrode, depleting the bulk layer of electrons and creating an electric field within its volume. A charged particle which passes through the solid-state detector causes ionization which in-turn produces electron-hole pairs. The electric fields within the microstrip detector drift electrons and holes to oppositely charged electrodes. In this manner, a silicon microstrip detector acts like a solid-state drift chamber and provides very high position resolution and fast detector readout.

The information recorded with the SVX detector is used in the reconstruction of secondary vertices, displaced from the primary vertex, which come from the decay of heavy-flavor particles such as hadrons which contain bottom and charm quarks 
and are known to have relatively short lifetimes as compared to the lifetimes of lightflavor mesons. ${ }^{7}$ The fine position resolution and fast readout time offered by the use of silicon microstrip detectors makes them perfect for finding displaced secondary vertices due to the decay of these relatively short-lived hadrons. The ability to reconstruct secondary vertices is critical for the algorithm exploited by this analysis for the identification of $b$ quarks from the top decay $t \rightarrow W b$ (see Section 3.4). Tracking information is provided by the silicon detector out to a pseudorapidity value $|\eta|<2.0$ and this particular subsystem is comprised of three cylindrical barrels, each consisting of five concentric layers of silicon microstrip detectors. All of the SVX microstrip detectors in these five layers are double-sided with one side to provide measurements in the $r$ - $\phi$ plane. Three of the five layers have a second side of sensing strips running perpendicular to the axial direction to provide measurements in the $r$ - $z$ plane, (known as " $90^{\circ}$ stereo" measurements). The remaining two layers have a second side of sensing strips tilted at an angle of $1.25^{\circ}$ with respect to the strips on the first side, for what are known as "small angle stereo" measurements. In principle, these stereo measurements allow for excellent pattern recognition and three-dimensional $(x, y, z)$ vertex reconstruction. Although not yet implemented at the time that the measurement described in this thesis was conducted, the $z$-position information will undoubtedly be used for other analyses in the near future. Averaged over all central $t \bar{t}$ candidate decay tracks which are known to have high transverse momentum, the impact parameter resolution achieved with the SVX is $24.8 \mu \mathrm{m}$ [42]. The SVX is excellent for secondary-vertex detection very close to the interaction point, and therefore is ideal for finding events which contain $b$-quarks.

The ISL microstrip sensors are very similar to those which make up the SVX. Similar to the SVX layers, the ISL sensors have one side for $r$ - $\phi$ (axial) measurements

\footnotetext{
${ }^{7}$ Due to the $b$ lifetime of $\sim 1.5 \mathrm{ps}$, B-mesons will travel $\sim 450 \mu \mathrm{m}$ before decaying. By comparison, light-flavor measons (like kaons and pions) tend to have much longer lifetimes-on the order of tens of nanoseconds.
} 
and a second side for small angle stereo measurement capabilities. The ISL tracking detector is comprised of three layers of concentric silicon microstrip detectors and is located radially just outside of the SVX subsystem. Centered around $z=0$, one layer of the ISL is radially positioned $22 \mathrm{~cm}$ from the interaction point. Two additional layers of microstrip detectors are positioned to match the high- $\eta$ coverage of the CDF plug calorimeter; these layers reside at radii of 20 and $28 \mathrm{~cm}$. The purpose of the ISL is to improve the efficiency of three-dimensional tracking in the central region and to add additional stand-alone silicon coverage in the forward (high- $\eta$ ) region.

It is worth noting that one additional layer of radiation-hard, single-sided, silicon detector called "Layer-00" exists closest to the beam pipe. Layer-00 employs state-ofthe-art silicon detector technology that enables excellent signal-to-noise performance even after extreme doses of radiation. Together with Layer-00 and the ISL there are a total of 8 layers of silicon between $1.0<\eta<2.0$. Layer- 00 is the newest of all the CDF II tracking systems and was not used in this analysis.

Because the SVX and ISL detectors are excellent for tracking, $b$-quark identification and aid in lepton identification, they are two of the most important detector systems at CDF for this analysis.

An integral part of the CDF tracking system, the Central Outer Tracker (COT) [41] is an open cell drift chamber contained within the volume of the superconducting solenoid. 30,240 gold-plated tungsten wires ${ }^{8}$ make up the sense-wires for channels in eight "superlayers", and together they provide 96 track measurements between radii of $44 \mathrm{~cm}$ and $132 \mathrm{~cm}$. The COT provides charged particle tracking with high efficiency in the region $|\eta| \leq 1.0$ (see Fig. 2.3), just outside of the two silicon trackers. Four "axial" superlayers run parallel to the beamline ( $z$-axis) which provide $r$ - $\phi$ information. The remaining four superlayers are positioned at a tilt of $2^{\circ}$ with respect to the $z$-axis to provide stereo information. These eight superlayers are enclosed inside of an air-tight

\footnotetext{
${ }^{8}$ Wireplane testing was carried out at the University of Illinois, see Appendix B.
} 
chamber, which is filled with a 50\%/50\% mixture of argon (Ar) and ethane $\left(\mathrm{C}_{2}-\mathrm{H}_{6}\right)$ gasses. Charged particles which traverse the COT drift chamber interact with the gas and leave behind an ionization trail, electrons in this trail travel along the electric field lines toward the sense wires. Charges which drift onto the sense wires generate electric signals. It is the time of arrival of these signals that allow measurements of the particle's trajectory. Electrons from the ionization are coaxed into drifting toward the sense wires through their interaction with an electric field created by voltages on the wires. An avalanche of electrons around the sense wire is registered as a pulse, and the drift time is converted into a distance of closest approach of the particle to the sense wire. Three-dimensional reconstruction of the path of the charged particle is then possible using these distance of closest approach measurements. The average drift velocity in the COT is $\sim 100 \mu \mathrm{m} \mathrm{ns}^{-1}$ and the average hit position resolution is approximately $140 \mu \mathrm{m}$ [38]. The COT has a $p_{T}$ resolution of $\delta p_{T} / p_{T}^{2} \simeq 0.15 \%$ $(\mathrm{GeV} / c)^{-1}, \cot \theta$ resolution of $6 \times 10^{-3}$, impact parameter and $z_{0}$ resolutions of $\sim 600$ $\mu \mathrm{m}$ and $\sim 5 \mathrm{~mm}$, respectively.

To correlate silicon tracking hit information with COT tracks an "outside-in" algorithm is used. This algorithm starts with a silicon hit in the $r$ - $\phi$ plane and the COT detector tracks are extrapolated back into the volume of the silicon tracking detectors. During each step of this extrapolation, multiple scattering considerations determine the region in which the algorithm looks for a track. Once a reconstructed track is found in the $r$ - $\phi$ plane, the $z$ position information for the silicon detector is added to the track. As mentioned earlier, the variable used to denote the point of origin of a track along the $z$-axis is known as " $z_{0}$ " [43]. Using the COT in conjunction with tracking information from the silicon detectors improves impact parameter and $z$ position measurements.

When track information from the SVX and ISL are used in conjunction with that from the Central Outer Tracker we expect to achieve $b$-tagging with an efficiency of 
$\sim 60 \%$ in $t \bar{t}$ events over the region $|\eta| \leq 1.0[44]$.

\subsubsection{Calorimetry}

The purpose of CDF's calorimetry is to measure the kinetic energy deposited by particles observed in the detector. Immediately outside of the solenoid volume and surrounding the tracking system, CDF uses segmented scintillator-based sampling calorimetry for the measurement of kinetic energy flow of interacting particles in the region $|\eta| \leq 3.64$ (see Fig. 2.3). The calorimeter is divided into two segments, each providing separate measurements for both electromagnetic (EM) and hadronic (HAD) energies. The EM section of calorimeter is closer in radius to the interaction point than the HAD section and is primarily used for the identification of electrons and photons. The HAD component of the calorimeter is where the kinetic energy deposited by hadronic particles in high-energy jets is measured. Both components use a series of scintillator with metal absorber layers. Light from the scintillator is collected by a light-pipe and a wavelength shifting fiber that directs the energy into a photomultiplier tube for detection. The EM and HAD sections of the calorimeter are segmented in $\eta$ and $\phi$ and are projective, which means that they point back to the nominal interaction region.

Bremsstrahlung, the process by which electrons (due to their low mass) loose energy by radiating photons while being decelerated in the Coulomb field of an atomic nucleus, causes high-energy electrons to loose energy in the form of electromagnetic

radiation. The mean distance over which the high-energy particle loses all but $\frac{1}{e}$ of its energy is known as one radiation length and is denoted by the variable $X_{0}$, given in $\mathrm{gm} \cdot \mathrm{cm}^{-2}$. The design of the CDF EM calorimeter is in terms of these radiation lengths, so it is more appropriate to discuss calorimeter construction in terms of $X_{0}$ rather than a more standard unit like meters. Through the use of a multi-layered sandwich of scintillating polystyrene and a $\mathrm{Pb}$ absorber, the EM calorimeter [45] has a 
thickness of $21 X_{0}$, or 21 radiation lengths worth of material. The central and end-wall calorimeters cover the pseudorapidity range $|\eta|<1.1$ and $|\eta|<1.3$, respectively. The energy resolution of the central EM calorimetry is $13.5 \% / \sqrt{E_{T}} \oplus 2 \%$, while the resolution of the plug electromagnetic calorimeter is $16 \% / \sqrt{E_{T}} \oplus 1 \%$ [46]. The first layer of the EM calorimeter (i.e., that closest to the interaction point) is made of a thicker scintillator in addition to a wire chamber which acts as a Calorimeter Pre-showeR detector (CPR). The CPR samples the early development of an electromagnetic shower within the solenoid. Behind the CPR, located in at a depth of roughly $6 X_{0}$, is the shower maximum detector (the "Shower Max" detector is also known as the CES) which is a system of proportional chambers with strip and wire readouts. The CES is a position detector used to match tracks found in the tracking systems to calorimeter energy deposits and provides shower shape and position information in both $z$ and $r-\phi$.

The thickness of the hadronic calorimeter is best described in terms of the pion nuclear absorption length, $\lambda_{0}$ (given in $\mathrm{gm} \cdot \mathrm{cm}^{-2}$ ), rather than radiation lengths. The central HAD detector [47] is comprised of 23 layers of acrylic scintillating material interspersed with Fe absorber. The central region of the HAD calorimeter contains approximately $4.5 \lambda_{0}$ of material. The more forward regions of the HAD calorimeter (the "plug" region) are $7 \lambda_{0}$ thick. The energy resolution of the central and plug hadron calorimeters is $74 \% / \sqrt{E_{T}} \oplus 4 \%$. The central and plug calorimeters cover the pseudorapidity ranges $|\eta|<1.1$ and $1.1<|\eta|<3.64$, respectively.

Both components of the calorimeter detectors are segmented in $\eta$ and $\phi$, providing EM shower and HAD projective tower segmentation. The tower size, $\Delta \eta \times \Delta \phi$, for the central and forward calorimeters are $0.1 \times 15^{\circ}$ and $0.1 \times 5^{\circ}$, respectively. Together these subsystems measure the net kinetic energy flow, and in conjunction with COT track-matching have proven to be a very powerful analysis tool. 


\subsubsection{Muon Detectors}

The muon systems at CDF reside outside of the calorimetry. Muon detection at CDF is done through the use of four layers of single wire drift chambers ${ }^{9}$ backed up by scintillator counters for fast timing. Collectively, these large arrays of drift chambers provide coverage for $|\eta| \leq 1.5$ and nearly complete coverage in $\phi$. The central-most array of drift chambers, the Central Muon (CMU), are able to determine the position of the track along the sense-wire in $z$. Being positioned furthest from the interaction point, the muon detectors are shielded with a significant amount of steel from the hadronic calorimeter, the magnet return yoke, and additional steel shielding intended to reduce hadronic punch-through. High $p_{T}$ muons $(\sim 1 \mathrm{GeV} / \mathrm{c})$ are minimum ionizing particles, which means that they are capable of traveling many hadronic interaction lengths before stopping. Muon chambers record hits along the path of the muon through the detector. Information from the muon detectors, when used in conjunction with information from the COT, results in excellent muon identification and accurate muon momentum information for use in data analysis. Muon detection is critical for the analysis presented here, as it will aid in the collection of about half the data set.

Four systems of scintillators and proportional wire chambers are used for detection of muons at CDF. Overall, the range for muon detection is $|\eta| \leq 2.0$. Beginning with the system closest to the interaction point and moving outward, these systems are the Central MUon (CMU), Central Muon uPgrade (CMP), Central Scintillator uPgrade (CSP), Central Muon eXtension (CMX), Central Scintillator eXtension (CSX), Toroid Scintillator Upgrade (TSU), Barrel Muon Upgrade (BMU) and the Barrel Scintillator Upgrade (BSU). The CMU/CMP/CSP detectors cover the range of $|\eta|<0.6$. The CMX/CSX detectors extend this coverage into the range $0.6 \leq|\eta|<1.0$ and the $\mathrm{TSU} / \mathrm{BMU} / \mathrm{BSU}$ systems cover the range $1.0 \leq|\eta| \leq 2.0$. Most of these systems

\footnotetext{
${ }^{9}$ The Central Muon (CMU) and Central Muon Upgrade (CMP) drift chambers were built at the University of Illinois. The muon calibration system for the CMP chambers was created at the University of Illinois, see Appendix C.
} 


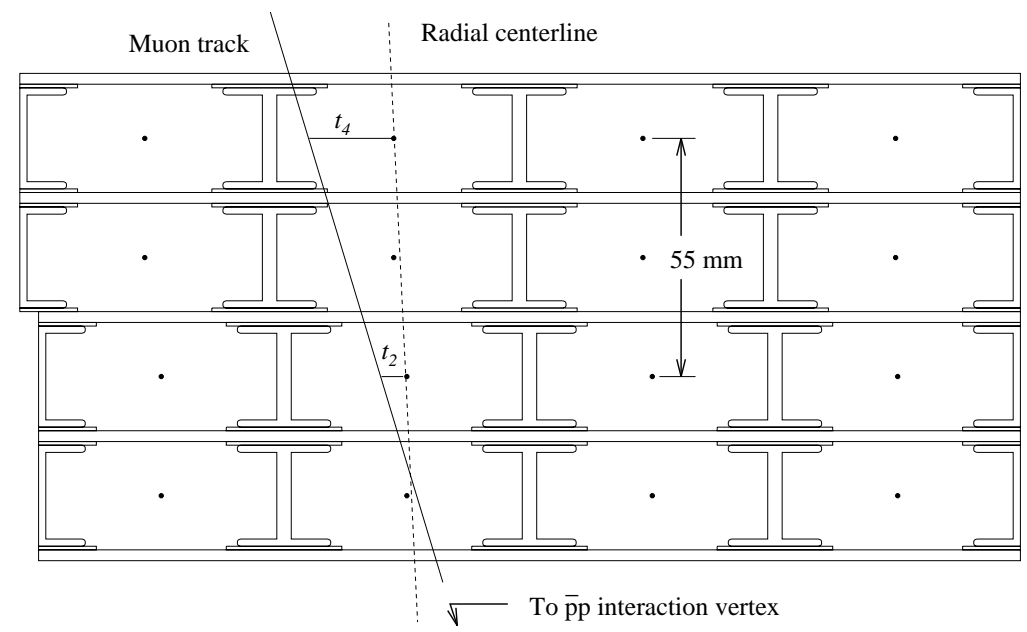

Figure 2.4: Cross section of a CMU module showing radial alignment of alternate wires. The time difference is used at the trigger level to obtain a crude momentum measurement. The module subtends $5^{\circ}$ in $\phi$.

were upgraded between Run I and Run II, or as in the case of the TSU/BMU/BSU were entirely new. The data used in the analysis presented in this thesis includes muons detected in the CMU, CMP and CMX. This analysis does not include muons detected by the BMU since a trigger was not in place at the time the dataset was collected. The BMU triggers on muons out to an $|\eta|$ value of 1.5 , this value was chosen to match tracking constraints.

The CMU chambers (see Figure 2.4) are located behind the central calorimeter, which provides $\sim 5.5$ absorption lengths of material for muons. The CMU detector is capable of detecting muons with $p_{T}>1.4 \mathrm{GeV} / \mathrm{c}$, which is the minimum momentum needed to get through the calorimeter. The CMP detectors form a box around the CMU and are located behind an additional $60 \mathrm{~cm}$ of steel absorber $\left(3.5 \lambda_{0}\right)$ which reduces hadronic punch-through by a factor of 20. The CMP detector can detect muons with $p_{T}>3.0 \mathrm{GeV} / \mathrm{c}$. The CMX muon chambers are located on the east and west ends of the CDF detector $( \pm z)$ and provide nearly complete coverage in $\phi$. The CMX drift tube chambers are backed by the CSX scintillating counters. On their way toward the CMX chambers, muons pass through steel absorber from the hadron 
calorimeter, the magnet return yoke, and the steel of the detector support structure.

Muon candidates are found by identifying hits in at least four layers of the muon chambers; the presence of a penetrating muon is reconstructed as a line segment or "stub" in one of these four-layer stacks. Isolated tracks which extrapolate to these stubs are known as muon candidates.

\subsubsection{Trigger System}

With the high instantaneous luminosity and great frequency of collisions at B0, it would be impossible for CDF to record and analyze every event. There is simply not enough disk space or time to record every single event observed inside of the CDF detector. The Tevatron delivers events to $\mathrm{CDF}$ at a rate of about $2.5 \mathrm{MHz}$ (once every $396 \mathrm{~ns}$ ) and the average event size is approximately $250 \mathrm{kB}$. At this frequency and event size, the bandwidth is $\sim 0.6 \mathrm{~TB} \cdot \mathrm{s}^{-1}$. To reduce the amount of data that is recorded and physically stored for future offline analysis to a manageable size, CDF uses an online three-tier trigger system that filters out many of the less-interesting events based on particle identification and measured kinematical information. The CDF II trigger system was designed to be "dead-timeless," which means that the CDF detector is capable of processing an event even if it is still busy with the preceding one.

All of the CDF detector subsystems are read out into this three-tier trigger system, depicted in Figure 2.5. At each level of the trigger, programmed electronics examine the data coming from the various subsystems to check if basic requirements imposed by the trigger have been met, at which point an event is deemed interesting enough to be passed on to the next level of scrutiny. The three levels of the CDF trigger system are sequential, meaning that if an event does not pass the Level-1 requirements it will never be considered by Level-2. Similarly, an event which fails Level-2 trigger criteria, will not get passed on to Level-3. 


\section{Dataflow of CDF "Deadtimeless" Trigger and DAQ}

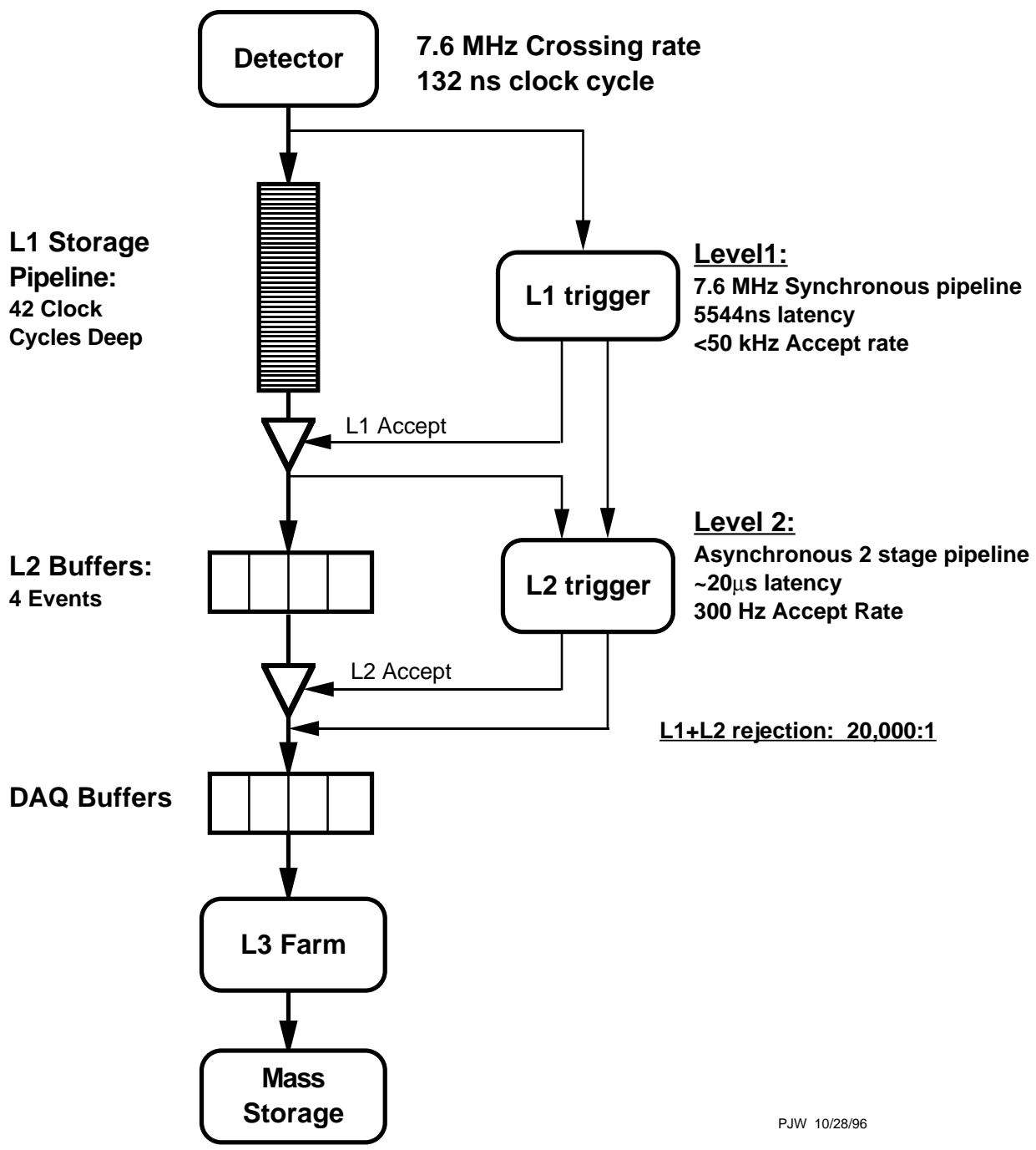

Figure 2.5: A block diagram of the CDF II data flow [38]. This flow diagram depicts a storage pipeline 42 clock cycles deep (for a $132 \mathrm{~ns}$ clock cycle and a $7.6 \mathrm{MHz}$ bunch crossing rate). The current configuration of the Tevatron delivers events to CDF at a rate of $2.5 \mathrm{MHz}$, which is a $396 \mathrm{~ns}$ clock cycle; with this configuration the Level-1 pipeline is capable of storing 14 clock cycles. 
The first tier in the trigger system, Level-1, collects data from the individual detector subsystems. Level-1 is capable of making a decision whether to accept or reject an event based on track information, calorimeter energy deposition and muon hit information. This first level of the trigger system was designed with a storage buffer 14 clock cycles deep (with 396 ns between bunch crossings) and arrives at a decision about any particular event before that event falls out of the pipeline. Within about $4 \mu$ s the Level-1 trigger has made a decision whether or not to pass a particular $p \bar{p}$ collision on to the second tier of the trigger system. After passing through the Level-1 trigger, the data rate has been reduced from the original $2.5 \mathrm{MHz}$ down to just under $20 \mathrm{kHz}$. The relevant Level-1 triggers for the top analysis are the muon and calorimeter triggers (discussed further in Chapter 3). The muon triggers use the fast output of the CMU, CMP and CMX detectors to identify muon candidates and match muon chamber hits with tracks in the COT. The calorimeter triggers separately sum the electromagnetic and hadronic energy in trigger towers [48, 49] of $(\Delta \eta=0.2) \times\left(\Delta \phi=15^{\circ}\right)$. The electromagnetic or hadronic energy in a trigger tower must pass a certain threshold to be accepted.

Level-2, the next tier in the CDF trigger system, has four local buffers and is asynchronous with a decision time of about $20 \mu$ s per event. Level-2 is capable of of making rejections based on track information [50] and calorimeter energy. After a Level-1 accept, the data on each front-end card is read out and transferred into one of the Level-2 buffers. Level-2 is capable of using CES information from the calorimeter for more refined identification of electrons and photons, as well as using a clusterfinding algorithm programmed into the Level-2 calorimeter for jet reconstruction. Acceptance from Level-2 flags an event, which is then collected by data acquisition buffers. Once collected the event is passed on to Level-3 via a network switch. Level-2 further reduces the bandwidth coming out of Level-1 by nearly a factor of 100; passing data on to Level-3 at a rate of $\sim 300 \mathrm{~Hz}$. 
The final stage in the trigger system, Level-3, is a congregation of nearly $300 \mathrm{CPU}$ nodes running software that is easily modified for optimization, which assemble and analyze each data event in greater detail. The Level-3 trigger algorithms perform a simplified version of the event reconstruction that is used in offline data analysis. Once an event is accepted by Level-3 it is passed on to the data-logger subsystem which sends the event on to be written for permanent storage. The triggers applied at Level-3 reduce the stream of data by roughly a factor of two. In the end $\sim 20 \mathrm{MB} \cdot \mathrm{s}^{-1}$ are written out to disk and tape. A maximum rate of $75 \mathrm{~Hz}$ can pass Level-3 and be written to tape. 


\section{Chapter 3}

\section{$t \bar{t}$ Event Selection}

The analysis discussed in this thesis measures the fraction of longitudinal $W$ boson polarization, $F_{0}$, in top quark decays using the distribution of the quantity $\cos \theta^{*}$ in $t \bar{t}$ candidate events. Reconstructing the $\cos \theta^{*}$ distribution when both $W \mathrm{~s}$ in $t \bar{t}$ events decay into a pair of quarks is quite challenging, as we have great difficulty determining the original quark and anti-quark from the hadronic jets that we see in the detector. In the case of dilepton events, where both $W$ s from a $t \bar{t}$ pair decayed leptonically, we are unable to reconstruct the $t \bar{t}$ event unambiguously since we lack sufficient information about the momentum vectors of the two neutrinos. Due to these difficulties, I have excluded the all-hadronic and dilepton decay channels from this analysis. I require a data sample where the $W$ boson from one top quark decay in the event has decayed leptonically $(t \rightarrow W b \rightarrow l v b)$ and the $W$ from the second top quark decay has decayed hadronically $\left(t \rightarrow W b \rightarrow q \bar{q}^{\prime} b\right)$. This sort of event topology is known as the "leptonplus-jets" channel because the signature of such events is one high- $p_{T}$ charged lepton, large missing transverse energy due to the neutrino (denoted by the variable, $\mathbb{t}_{T}$ ) and ideally four hadronic jets-two jets from the hadronically decaying $W$, two from the hadronization of the $b$-quarks in the event. Approximately $33 \%$ of the $t \bar{t}$ events produced at CDF will have this decay signature. It is possible to fully reconstruct the lepton-plus-jets events, but it is difficult to match each hadronic jet to the quark which produced them. The technique used in this analysis for matching jets to the 
quarks which produced them is addressed in Chapter 4 .

The remainder of this chapter details the event selection criteria for the leptonplus-jets events used by this analysis. A discussion of the cut efficiencies and motivations for these selection criteria are briefly discussed here, but are well documented in $[44,51,52,53,54,55,56]$.

\subsection{Inclusive High- $p_{T}$ Electrons}

Only electrons detected in the central calorimeter region $(|\eta|<1.0)$ are considered. The electron is expected to deposit most of its energy in a single electromagnetic calorimeter tower, in addition to leaving a track in the COT which points to the associated cluster in the CEM. Additionally, the electron should have a well-defined transverse shower profile in the CES (this parameter is known as the "lateral shower profile", $\left.L_{s h r}\right)$. The lateral shower profile describes how well the energy deposited in the calorimeter matches that coming from an ideal electron.

\subsubsection{Electron Triggers}

For the collection of lepton-plus-jets events we impose the online requirement during data-taking that there exist at least one high-energy lepton in the event. We have online triggers for high-energy electrons and muons. The online electron triggers are discussed in this section, the online muon triggers are discussed later on in Section 3.2.1. These requirements are imposed by the CDF trigger system; the trigger system itself is discussed in further detail in Section 2.2.4. The trigger path for the accumulation of events in this dataset is ELECTRON_CENTRAL_18, used for collecting central high-energy electrons. More stringent event selection criteria are later applied offline after the data has already been written to disk or tape for further analysis.

The trigger path for high-energy electrons is comprised of three separate triggers, 
one at each level of the three-tier trigger system at CDF: L1_CEM8_PT8, L2_CEM16_PT8, and L3_ELECTRON_CENTRAL_18. They are summarized below.

- L1_CEM8_PT8 (Level-1)

- One or more depositions of energy in the central EM calorimeters with $E_{T} \geq 8 \mathrm{GeV}$; hadronic to electromagnetic energy ratio (HAD/EM) $\leq 0.125$

- One or more COT tracks found having $p_{T} \geq 8.34 \mathrm{GeV} / \mathrm{c}$

- L2_CEM16_PT8 (Level-2)

- One or more depositions of energy in the central calorimeter with $E_{T} \geq 16$ $\mathrm{GeV} ; \mathrm{HAD} / \mathrm{EM} \leq 0.125$

- A requirement of $|\eta| \leq 1.317$ is imposed on the cluster centroid (also referred to as the "seed"), thus guaranteeing that the cluster is located in the central portion of the calorimeter

- Requires a match between tracks found in the COT at Level-1 to the cluster within a window of $\phi$, as determined by multiple scattering interactions in the detector

- Track $p_{T} \geq 8.34 \mathrm{GeV} / \mathrm{c}$

- L3_ELECTRON_CENTRAL_18 (Level-3)

- Cluster $E_{T}$ is re-calculated by using the primary interaction location as found by the track matched to the cluster

- Cluster $E_{T} \geq 18 \mathrm{GeV}$

- Track $p_{T} \geq 9.00 \mathrm{GeV} / \mathrm{c}$

- A cut is made on the lateral shower profile of the cluster $\left(L_{s h r}<0.4\right)$ 


\subsubsection{Offline Electron Selection}

Selection requirements applied to data offline are cuts which are tighter than those made online through the use of the trigger during data collection. This is done to reduce the number of background events contained in our set of $t \bar{t}$ candidate events. The offline selection criteria are listed in detail below, and summarized at the end of this chapter in Table 3.1 .

- $E_{T} \geq 20 \mathrm{GeV}$

- Minimum energy $E_{T}$ deposited in the calorimeter of $20 \mathrm{GeV}$.

- $p_{T} \geq 10 \mathrm{GeV} / \mathrm{c}$

- Minimum COT track momentum (transverse) of $10 \mathrm{GeV} / \mathrm{c}$.

- $E_{H A D} / E_{E M}<0.055+0.00045 \times E$

- A tightened requirement on the $E_{H A D} / E_{E M}$ ratio to be less than $\sim 0.055$. This cut further reduces the number of jets which are misidentified as electrons. In general, electromagnetic showers occur within the first few radiation lengths of the CEM and hence deposit little energy in the hadronic sections of the calorimeter.

- $E / p<2.0\left(\right.$ if $\left.E_{T}<100 \mathrm{GeV}\right)$

- The ratio of the energy deposited by an electron in the CEM to the electron's momentum is expected to be $\sim 1$.

- $p_{T} \geq 50\left(\right.$ if $\left.E_{T} \geq 100 \mathrm{GeV}\right)$

- This suppresses high $p_{T}$ hadrons that fake electrons.

- $Q \times \Delta x>-3.0$ and $<1.5 \mathrm{~cm}$ 
- This cut uses the electron candidate charge, $Q$, and $\Delta x$ of the track to confirm that we are indeed using a CES track which falls within the allowed extrapolation window in $x$.

- $|\Delta z|<3.0 \mathrm{~cm}$

- The variable $\Delta z$ is the distance in the $r-z$ plane between the COT track position and the CES chamber position. We extrapolate the COT track into the CES region of the calorimeter and check if there are any CES hits that lie within this window of $\Delta z$.

- $\chi_{\text {strip }}^{2}<10$

- The $\chi^{2}$ from a comparison of the CES shower profile to our expectations from test electrons. This is a cut on the quality of the shape of the cluster.

- $L_{s h r}<0.2$

- The variable $L_{s h r}$ is the "lateral shower profile" for electrons. This variable compares the energy of the seed tower in the electromagnetic cluster to that of adjacent CEM towers. We require that the lateral shower profile look similar to that seen with test beam electrons. We define $L_{s h r}$ as: $L_{s h r}=0.14 \times \sum_{i} \frac{E_{i}^{o b s}-E_{i}^{e x p}}{\sqrt{(0.14 \sqrt{E})^{2}+\sigma_{E_{i}^{2}}^{\text {exp }}}}$, where the sum is taken over towers adjacent to the seed tower, $E_{i}^{o b s}$ is the energy deposited in the CEM tower by the electron candidate, $E_{i}^{e x p}$ is the energy expected from the test beam electrons, $0.14 \sqrt{E}$ is the energy resolution of the CEM, and $\sigma_{E_{i}^{e x p}}$ is the uncertainty in $E_{i}^{e x p}$. When the match with the test beam electrons is exact, $L_{s h r}=0$.

- \# COT Axial Segments $\geq 3$ 
- COT tracks have a set of minimum requirements imposed on them (in both axial and stereo dimensions) for the number of good segments and the number of hits per segment.

- \# COT Stereo Segments $\geq 3$

- $\left|\Delta z_{0}\right|<60 \mathrm{~cm}$

$-\Delta z_{0}$ is the difference between the displacement along the beamline of the primary vertex (see Section 3.6) in a given event, and the displacement of the electron track along the beamline. A cut on $\Delta z_{0}$ guarantees that we use a COT track which originates in only the optimal portions of the tracking chamber.

- Isolation

- An electron is expected to leave a well collimated energy deposit in the calorimeters. Jets however, tend to leave large spread-out calorimeter deposits which are poorly collimated. We are able to reject a large number of jets that fake electrons by looking at a variable called "isolation". The electron candidate isolation is calculated by measuring all of the $E_{T}$ in both the hadronic and electromagnetic calorimeters within a cone ${ }^{1}$ of $\Delta R \leq 0.4$ around the candidate. The isolation is defined as: iso $\equiv \frac{E_{T, \Delta R<0.4}-E_{T, e l e}}{E_{T, \text { ele }}}$, where $E_{T, \Delta R \leq 0.4}$ is the transverse energy within a cone of 0.4 and $E_{T, \text { ele }}$ is the transverse energy of the electron candidate.

- Fiducial cuts

- For electrons, fiduciality cuts require that a cluster centroid falls within the region of the detector well covered by the CES, and in an area of the

\footnotetext{
${ }^{1} \Delta R$ is defined as: $\sqrt{\left(\eta_{\text {tower }}-\eta_{\text {centroid }}\right)^{2}+\left(\phi_{\text {tower }}-\phi_{\text {centroid }}\right)^{2}}$
} 
detector where the energy response from CEM phototubes is well understood. Additionally, we require that the shower position, as measured by the CES, be a good distance away from the CEM wedge boundaries and any known inactive regions.

- Conversion removal

- Photons which interact with matter are capable of producing electronpositron pairs. These electrons are known as "conversion" electrons. Conversion electrons contaminate our sample when they fake the charged lepton from $W$ decay. A conversion removal algorithm is applied to all $t \bar{t}$ candidate events which contain an electron that passed the tighter set of offline cuts. This algorithm looks for electrons which may have come from photon conversions within detector material that resides between the interaction point and the calorimetry. A conversion electron is expected to have a track initially parallel to it's own due to the partner electron, with an angular separation between itself and the partner track in the $x-y$ plane of $<0.2$ and a separation in the $z$ direction of $<0.004$. Any electron candidate with such a neighboring track is labeled as a conversion and is rejected from consideration as the tight lepton in that event.

\subsection{Inclusive High- $p_{T}$ Muons}

Muon candidates are expected to have a track in the COT which points to hits in the muon chambers. Muons are almost minimum ionizing particles, hence the energy deposited by muons in the calorimeter is expected to be only a few $\mathrm{GeV}$, independent of momentum. 


\subsubsection{Muon Triggers}

As discussed previously in Section 2.2.3, there are three centrally-located muon detectors: CMU, CMP and CMX. Muons in this dataset must follow the MUON_CMUP18 trigger path or the MUON_CMX18 trigger path.

The trigger path for high-energy muons observed in both the CMU and CMP detectors, MUON_CMUP18 is comprised of three separate triggers, one at each level of the three-tier trigger system at CDF: L1_CMUP6_PT4, L2_TRK8_L1_CMUP6_PT4, and L3_MUON_CMUP_18.

- L1_CMUP6_PT4 (Level-1)

- A muon stub in the CMU detector with $p_{T} \geq 6 \mathrm{GeV} / \mathrm{c}$

- A track in the COT detector with $p_{T} \geq 4.09 \mathrm{GeV} / \mathrm{c}$

- Requires an additional "stub" in the CMP detector

- Matching is done at Level-1 between the muon "stubs" and the COT track, within a $\phi$ window just a few degrees wide

- L2_TRK8_L1_CMUP6_PT4 (Level-2)

- One or more tracks in the COT detector ranging between $\phi$ of 0 and $\pi$

- A track in the COT detector with $p_{T} \geq 8 \mathrm{GeV} / \mathrm{c}$

- L3_MUON_CMUP_18 (Level-3)

- a match between tracks in the COT and muon stubs in both the CMU and CMP detectors, allowing a window in $r-\Delta \phi$ less than $10 \mathrm{~cm}$ for CMU stubs and $20 \mathrm{~cm}$ for CMP stubs

- COT track with $p_{T} \geq 18 \mathrm{GeV} / \mathrm{c}$ 
The trigger path for high-energy muons observed in the CMX detector, MUON_CMX18, is comprised of three separate triggers, one at each level of the three-tier trigger system at CDF: L1_CMX6_PT8_CSX, L2_AUTO_L1_CMX6_PT8_CSX, and L3_MUON_CMX18.

- L1_CMX6_PT8_CSX (Level-1)

- A muon stub in the CMX detector with $p_{T} \geq 6 \mathrm{GeV} / \mathrm{c}$

- Hits are required in the CSX detector

- A track is required in the COT which is passing through at least 4 layers of the detector and with $p_{T} \geq 8.34 \mathrm{GeV} / \mathrm{c}$

- L2_AUTO_L1_CMX6_PT8_CSX (Level-2)

- This trigger is an auto accept trigger; no additional requirements are imposed on the data at this level

- L3_MUON_CMX18 (Level-3)

- COT detector tracks matched to a CMX stub in a window of $r-\Delta \phi$ of less than $10 \mathrm{~cm}$

- A COT track with $p_{T} \geq 18 \mathrm{GeV}$

\subsubsection{Offline Muon Selection}

Position information from the COT and muon chambers can be used together to extrapolate the path of a muon through the CDF detector. More stringent requirements regarding the amount of electromagnetic or hadronic energy associated with muon candidates are applied offline than those imposed online during data collection. The offline selection criteria are listed in detail below, and summarized in Table 3.1 at the end of this chapter.

- $p_{T} \geq 20 \mathrm{GeV} / \mathrm{c}$ 
- Muons from $W$ decay are expected to have high- $p_{T}$. Our lower bound of $20 \mathrm{GeV} / \mathrm{c}$ on the muon $p_{T}$ reflects this expectation.

- $E_{E M}<\max (2.0,2.0+0.0115 \times(p-100.0)) \mathrm{GeV}$

- The energy deposited by a muon passing through a CEM tower must be less than 2.0 or $(2.0+0.0115 \times(p-100.0)) \mathrm{GeV}$, whichever is a maximum. This ensures that muon candidates in the dataset are consistent with our expectations for minimum ionizing particles.

- $E_{H A D}<\max (6.0,6.0+0.0280 \times(p-100.0)) \mathrm{GeV}$

- The energy deposited by a muon passing through a central hadronic calorimeter tower must be less than 6.0 or $(6.0+0.0280 \times(p-100.0)) \mathrm{GeV}$, whichever is a maximum. The threshold for the hadronic region of the calorimeter is higher than that for the electromagnetic region because the hadronic region contains more material for the muon to interact with.

- $|\Delta x|_{C M U}<3.0 \mathrm{~cm}$

- This is a COT - muon detector (CMU) matching requirement. The extrapolation of a COT track to the muon chambers must fall within $\Delta x$ of the associated hits in the CMU detector.

- $|\Delta x|_{C M P}<5.0 \mathrm{~cm}$

- This is a COT - muon detector (CMP) matching requirement. The extrapolation of a COT track to the muon chambers must fall within $\Delta x$ of the associated hits in the CMP detector.

- $|\Delta x|_{C M X}<6.0 \mathrm{~cm}$ 
- This is a COT - muon detector (CMX) matching requirement. The extrapolation of a COT track to the muon chambers must fall within $\Delta x$ of the associated hits in the CMX detector.

- $\left|\Delta z_{0}\right|<60 \mathrm{~cm}$

- $\Delta z_{0}$ is the difference between the displacement along the beamline of the primary vertex (described in Section 3.6) in a given event, and the displacement of the muon track along the beamline. A cut on $\Delta z_{0}$ guarantees that we use a COT track which originates in only the optimal portions of the tracking chamber.

- $\left|d_{0}\right|$ if no silicon hits $<0.2 \mathrm{~cm}$

- The impact parameter, $d_{0}$, is used to help with the rejection of cosmic rays which pass through the CDF detector and might enter the dataset. $d_{0}$ is determined using the track which has been matched to the hits in the muon detector. The cut on impact parameter is looser for tracks which do not have silicon hits than for those which do.

- $\left|d_{0}\right|$ if silicon hits $<0.02 \mathrm{~cm}$

- \# COT Axial Segments $\geq 3$

- COT tracks have a set of minimum requirements imposed on them (in both axial and stereo dimensions) for the number of good segments and the number of hits per segment.

- \# COT Stereo Segments $\geq 3$

- Isolation $<0.1$

- Like electron candidates, muon candidates have a maximum value of isolation imposed on them. For muons, isolation is calculated the same as for 
electrons, although here we do not need to subtract the muon $p_{T}$. To measure muon isolation we measure all $E_{T}$ (both hadronic and electromagnetic) in a cone of $\Delta R \leq 0.4$ around the track which has been matched to hits in the muon detectors. The isolation is then determined as: $i s o \equiv \frac{E_{T, \Delta R<0.4}}{p_{T, \text { muon }}}$, where $p_{T, \text { muon }}$ is the $p_{T}$ of the muon track calculated using hit information from the COT and $E_{T, \Delta R \leq 0.4}$ is the transverse energy within a cone of $\Delta R \leq 0.4$

- COT exit radius (for CMX muons) $>140 \mathrm{~cm}$

- The exit radius requirement is imposed on CMX muons only, to guarantee that muons passing through the COT detector had the opportunity to pass through a minimum number of COT layers prior to being matched to hits in the CMX.

- Cosmic Veto

- Cosmics removal $[57,58]$ : The mesonic, or "hard", component of cosmic rays manifest themselves in the CDF detector as muons from pion decay in the upper atmosphere of the Earth. It is possible to reconstruct these muons in our detector, although they did not originate from the interaction point or come from the proton-antiproton collisions. Most cosmic rays pass through the CDF detector leaving two signatures in the muon detectors separated in $\phi$ by $180^{\circ}$ and separated in time as the muon passes from one end of the detector to the other. The cosmic veto algorithm is capable of rejecting cosmic rays by cutting on the $\Delta \phi$ between a reconstructed muon and any other muon stub found in the detector while considering the timing information obtained for the calorimetry and tracking detectors. 


\subsection{Jet Selection}

The quarks which come about during proton-antiproton collisions through gluon splitting, the decay of $W$ and $Z$ bosons, or many other processes, eventually hadronize and manifest themselves in the CDF detector as showers of neutral and charged particles which leave energy deposits in various sections of the calorimeter. High-energy physicists call these showers of energy "jets." In a detector environment, jets are identified by using an algorithm that groups the individual calorimeter energy deposits in neighboring towers together into what is called a "cluster." Jets which have been used in this analysis are reconstructed offline using the JetClu tower clustering algorithm.

The JetClu algorithm organizes the energies measured in the calorimeter towers and assigns all towers with an energy greater than $3 \mathrm{GeV}$ as "seed" towers. Clustering begins with the highest $E_{T}$ tower in any given event, and the seed is taken to be the geometric center of the reconstructed jet. All neighboring towers within $\Delta R<0.4$ of the seed tower are grouped together into the same cluster. After all neighboring energy deposits within the specified cone of $\Delta R$ have been included, a new energyweighted centroid is calculated and the clustering algorithm begins again using the centroid as the new geometric center of the cone. Once JetClu has incorporated a tower into a cluster, that particular tower is excluded from being included in any other jet.

The lepton-plus-jets events used by this analysis are required to have four jets. All four of these jets may be "tight," or we could accept events where three jets are tight and one jet is "loose," where the $E_{T}$ requirement on one jet has been relaxed. Tight jets are defined as jets with $E_{T} \geq 15 \mathrm{GeV}$ and $|\eta|<2.0$ (the jet must be in the central or plug region of the calorimeter). Loose jets have the same $\eta$ requirement as the tight jets, however the $E_{T}$ requirement is relaxed down to $E_{T} \geq 8 \mathrm{GeV}$. Events

with three tight jets and one loose jet, or four tight jets are referred to as " 3.5 -jet 
events" and "4-jet events," respectively. The acceptance of 3.5-jet events is allowed because this analysis requires at least four high- $E_{T}$ jets in each event for the purpose of kinematic fitting (this is discussed further in Chapter 4). It is worth noting that jet energies need to be corrected for calorimeter mis-measurements before $E_{T}$ or $\eta$ cuts are applied (as discussed in Chapter 7).

\subsection{Secondary Vertex $b$-Tagging}

Most of the non- $t \bar{t}$ events which pass the lepton-plus-jets event selection criteria are not processes which contain any heavy flavor $b$ or $c$ quarks in the final state. A $b$ quark "tagging" technique, similar to one that was used to discover the top quark at CDF during Run I of the Tevatron, is used in this analysis. This method identifies $B$ hadron decays by looking for the displacement of a secondary vertex relative to the primary vertex. This is known as the "SecVtx" tagging algorithm. Secondary vertex $b$-tagging keeps more than half of the $t \bar{t}$ signal events while rejecting $\sim 95 \%$ of the background events. The $b$-tagging technique and the SecVtx algorithm are described in more detail elsewhere $[44,59]$. The efficiencies for this algorithm to tag a jet in detector and Monte Carlo data are not the same. Studies were carried out at CDF to determine these efficiencies as a function of jet $E_{T}$; the ratio of these two values averaged over jet $E_{T}$ is known as the "scale factor". The scale factor is well-fitted by a straight line and does not appear to depend on jet $E_{T}[44]$.

The lepton-plus-jets events observed in the data can be analyzed before or after the SecVtx tagging algorithm has been run over the data. The "pre-tagged" sample consists of events which pass the lepton-plus-jets event selection criteria, but have not yet had the $b$-tagging algorithm run over them. The pre-tagged sample is useful for background studies, in particular estimates of the non- $W$ QCD background (see Chapter 5). 


\subsection{Missing Transverse Energy}

In proton-antiproton collisions at the Tevatron, the total transverse energy $\left(E_{T}=\right.$ $E \cdot \sin \theta$, where $\theta$ is determined assuming that the interaction point is in the very center of the detector) should sum to zero since the colliding protons and anti-protons are known to have zero momentum in the transverse $(x-y)$ plane. For each of our $t \bar{t}$ candidate events, we take a vector sum of the transverse energy of all calorimeter towers and muons in the event. The difference between zero and this vector sum is taken as the missing transverse energy, $\mathbb{E}_{T}$. There are many sources of $\mathbb{E}_{T}$ : neutrinos in the event which escape the detector without having their energy measured directly, charged or neutral particles which escape the detector through cracks between calorimeter wedges or gaps in muon coverage, or even energy mis-measurement. ${ }^{2}$ In the lepton-plus-jets dataset, we expect events with missing transverse energy due to the neutrino from semi-leptonic top decay in $t \bar{t}$ events.

When tracks are reprocessed offline, a curvature correction has an impact on the muon $p_{T}$. Accordingly, we correct the $x$ and $y$ components of the $\mathbb{E}_{T}$ to account for this:

$$
\begin{aligned}
& \mathscr{E}_{T, x}=\not_{T, x}+\left(p_{T, \text { uncor }}-p_{T, \text { corr }}\right) \cdot \cos \left(\phi_{\text {uncor }}\right) \\
& \mathscr{F}_{T, y}=\not_{T, y}+\left(p_{T, \text { uncor }}-p_{T, \text { corr }}\right) \cdot \sin \left(\phi_{\text {uncor }}\right) .
\end{aligned}
$$

Up to this point, the $\not_{T}$ has been calculated using raw jet energies. The jet energies are corrected prior to the application of our $E_{T}$ cuts (see Chapter 7), so components of the $\mathbb{E}_{T}$ now need to be adjusted for the difference between the raw and the corrected jet energies. This is done for all jets, which after being corrected, have $E_{T} \geq 8.0 \mathrm{GeV}$

\footnotetext{
${ }^{2}$ If the true interaction point is off-center, the angle $\theta$ will be distorted and the calculated $E_{T}$ will be incorrect.
} 
and which lie in the pseudorapidity range $|\eta| \leq 2.5$ :

$$
\begin{aligned}
& \mathbb{E}_{T, x}=\mathbb{E}_{T, x}+\left(E_{T, \text { raw }}-E_{T, \text { corr }}\right) \cdot \cos \left(\phi_{\text {raw }}\right) \\
& \mathbb{E}_{T, y}=\mathbb{E}_{T, y}+\left(E_{T, \text { raw }}-E_{T, \text { corr }}\right) \cdot \sin \left(\phi_{\text {raw }}\right) .
\end{aligned}
$$

The final corrected $\mathscr{H}_{T}$ vector is determined simply by summing the above components in quadrature and then taking the square root, as below in Equation 3.5. During the offline analysis of the data, we impose a final cut of $\geq 20 \mathrm{GeV}$ on the corrected $\mathbb{E}_{T}$.

$$
\mathscr{H}_{T}=\sqrt{\mathscr{H}_{T, x}^{2}+\mathbb{H}_{T, y}^{2}}
$$

\subsection{Primary Vertex}

Some of the lepton-plus-jets selection criteria require knowing the position of the interaction point (i.e., where the proton and antiprotons are focused to collide), also known as the primary vertex. Calculations for $E_{T}$ as well as $\mathbb{E}_{T}$ rely on knowing the position in $z$ where the collisions occur on an event-by-event basis.

Initially, stand-alone tracking using information from only the silicon and COT detectors is used to determine the primary vertex $z$ position. A set of minimal quality requirements are imposed on both silicon and COT tracks; tracks which pass these requirements are then used by the tracking algorithm to triangulate vertices in the $r-z$ plane. There is a high fake rate for these vertices. Applying the primary vertex finder, ZVertexColl, imposes more stringent criteria on the number of tracks and the minimum $p_{T}$, hence cleaning up the stand-alone tracking vertices. ZVertexColl requires that vertices contain tracks with either silicon or COT hits associated with

them. The lepton-plus-jets dataset used by this analysis only considers vertices with 
at least two tracks that contain COT hits [60].

A point of origin along the $z$-axis, known as $z_{0}$, is assigned for all tracks in the event. If a track happens to pass within $1 \mathrm{~cm}$ of a silicon stand-alone vertex, or within $5 \mathrm{~cm}$ of a COT stand-alone vertex, it is considered to belong to that vertex. To determine the position of the vertex, the $z_{0}$ of all tracks for a particular vertex are weighted by their errors

$$
z=\frac{\sum_{i} z_{0, i} / \delta_{i}^{2}}{\sum_{i} 1 / \delta_{i}^{2}}
$$

where the sum is taken over all tracks that have been associated with a particular vertex, $z_{0, i}$ and $\delta_{i}$ are the $z_{0}$ and the associated error of the $i$ th track, respectively.

The PrimeVtx algorithm [42] is used to find the $x$ and $y$ coordinates of the primary vertex-this information is essential for the $b$-quark tagging algorithim. Starting from an input vertex with a given $x_{0}, y_{0}$ and $z_{0}$ position, the primary vertex is calculated on an event-by-event basis by PrimeVtx. This input primary vertex is the beamline position which has been measured during proton-antiproton collisions. Some requirements on $\Delta z$ and the impact parameter, $d_{0}$, are imposed on the tracks prior to being considered by the vertexing algorithm:

- $\left|z-z_{\text {vertex }}\right|<1.0 \mathrm{~cm}$

- $\left|d_{0}\right|<1.0 \mathrm{~cm}$ (with respect to the beamline)

- $\left|d_{0}\right| / \sigma<3.0$ (with respect to the beamline).

After passing these selection criteria the tracks are ranked by decreasing $p_{T}$. Only the highest $p_{T}$ tracks which pass the selection criteria (limited to no more than 50 tracks) are used in a fit to the primary vertex. The PrimeVtx algorithm removes the tracks with the worst fit; this is determined by requiring $\chi_{f i t}^{2}<10$. The algorithm continues this process of fitting and track rejection based on $\chi_{f i t}^{2}$ iteratively, until there are no tracks in the fit which have a $\chi_{f i t}^{2}>10$. The precision obtained in the 
determination of the vertex $x$ and $y$ coordinates is $\sim 25-30 \mu \mathrm{m}$, but strongly depends on the $z$ position of the vertex [61].

\subsection{The Lepton-plus-Jets Event Selection}

Several additional cuts are applied to the candidate events to purify the lepton-plusjets sample. These are listed in detail below, and can also be found at the end of this chapter in Table 3.1:

- One tight high- $p_{T}$ lepton

- A single tight electron or muon as described previously in Sections 3.1.2 and 3.2.2

- $\mathbb{E}_{T} \geq 20 \mathrm{GeV}$

- Missing transverse energy $\geq 20 \mathrm{GeV}$ (as discussed in Section 3.5)

- 3.5 or 4 jet events

- Requirement of at least one $b$-tagged jet

- $Z$ veto:

- The $Z$ veto criteria removes events in which the invariant mass of the charged lepton and another object in the event falls within a specific range of the $Z$ boson mass. It is the intention of this cut to eliminate events which come from the decays of $Z$ bosons, but which managed to pass the more general lepton-plus-jets selection criteria when one lepton from the decay of the $Z$ is lost. $Z$ bosons can mimic the signal of a $W$ boson in the CDF detector when one of the leptons from the $Z$ decay are misidentified. As an example, consider the decay $Z \rightarrow e e$ where one electron is included 
in the jet collection, or falls into a crack between calorimeter wedges. The veto rejects events where the tight lepton and any second object form an invariant mass between 76 and $106 \mathrm{GeV} / \mathrm{c}^{2}$, which corresponds to a \pm 15 $\mathrm{GeV} / \mathrm{c}^{2}$ window around the $91 \mathrm{GeV} / \mathrm{c}^{2} Z$ boson mass.

- Dilepton veto

- This is done to ensure that no $t \bar{t}$ candidate events which belong in the dilepton sample make it into the lepton-plus-jets event sample.

\subsection{Summary}

The number of events collected in the lepton-plus-jets channel at CDF was dependent on many factors, the largest of which were the center-of-mass energy of the collisions and most importantly the total integrated luminosity. 


\begin{tabular}{lc}
\hline \hline Variable & Value \\
\hline \hline & Global Event Quantities \\
\hline$Z$ and Dilepton Veto & Applied \\
$\mathbb{E}_{T}$ & $\geq 20 \mathrm{GeV}$ \\
$\#$ tight leptons & 1 \\
\hline & Jets \\
\hline$E_{T}$ & $\geq 15 \mathrm{GeV}$ ("tight" jet) \\
$E_{T}$ & $\geq 8.0 \mathrm{GeV}$ and $<15 \mathrm{GeV}$ ("loose" jet) \\
$|\eta|$ & $<2.0$ \\
\hline & Tight Electrons \\
\hline Fiducial and CEM & True \\
$E_{T}$ & $\geq 20 \mathrm{GeV}$ \\
$p_{T}$ & $\geq 10 \mathrm{GeV} / \mathrm{c}$ \\
$E_{H A D} / E_{E M}$ & $<0.055+0.00045 \times E$ \\
$E / p$ (if $\left.E_{T}<100 \mathrm{GeV}\right)$ & $<2.0$ \\
$p_{T}$ (if $\left.E_{T} \geq 100 \mathrm{GeV}\right)$ & $\geq 50$ \\
$Q \times \Delta x$ & $>1.5 \mathrm{and}>3.0 \mathrm{~cm}$ \\
$|\Delta z|$ & $<3 \mathrm{~cm}$ \\
$\chi_{s t r i p}^{2}$ & $<10$ \\
$L_{s h r}$ & $<0.2$ \\
$\#$ Axial Segments & $\geq 3$ \\
$\#$ Stereo Segments & $\geq 3$ \\
$\left|\Delta z_{0}\right|$ & $<60 \mathrm{~cm}$ \\
Isolation & $<0.1$ \\
Conversion $(\Delta \Lambda \leq 0.03$ and $\Delta r \leq 0.3)$ & False \\
\hline & Tight Muons \\
\hline Cosmic Veto & Applied \\
CMUP or CMX & True \\
$p_{T}$ & $\geq 20 \mathrm{GeV} / \mathrm{c}$ \\
$E_{E M}$ & $<\max (2.0,2.0+0.0115 \times(p-100.0))$ \\
$E_{H A D}$ & $<\max (6.0,6.0+0.0280 \times(p-100.0)$ ) \\
$|\Delta x|_{C M U}$ & $<3.0 \mathrm{~cm}$ \\
$|\Delta x|_{C M P}$ & $<5.0 \mathrm{~cm}$ \\
$|\Delta x|_{C M X}$ & $<6.0 \mathrm{~cm}$ \\
$\left|\Delta z_{0}\right|$ & $<60 \mathrm{~cm}$ \\
$\left|d_{0}\right|$ if no silicon hits & $<0.2 \mathrm{~cm}$ \\
$\left|d_{0}\right|$ if silicon hits & $<0.02 \mathrm{~cm}$ \\
$\#$ Axial Segments & $\geq 3$ \\
$\#$ Stereo Segments & $<0.1$ \\
Isolation & \\
COT exit radius (for CMX) & \\
\hline \hline
\end{tabular}

Table 3.1: Identification and kinematic cuts for electrons, muons and jets. 


\section{Chapter 4}

\section{Measurement Method}

This analysis measures the fraction of longitudinal $W$ bosons produced in top quark decays by analyzing the $\cos \theta^{*}$ distributions of $t \bar{t}$ candidate events. For each leptonplus-jets event, the quantity $\cos \theta^{*}$ is calculated from the $m_{l b}$ approximation as defined in Equation 1.13 using the isolated charged-lepton four-momentum and the fourmomentum of the jet chosen by a kinematic fitter as being the most probable match to the $b$ parton in top quark decays where $t \rightarrow W b \rightarrow l \nu b$. The fraction of longitudinal $W$ bosons is then extracted by comparing the reconstructed $\cos \theta^{*}$ distributions in data with different templates using a binned maximum likelihood fit.

\subsection{Event Reconstruction}

As was mentioned in Chapter 1, in the interests of obtaining the highest precision possible for this measurement it is important to correctly identify the leptonic- $b$ when using the $m_{l b}$ approximation for $\cos \theta^{*}$. At the early stages of this analysis, tests were performed on $t \bar{t}$ Monte Carlo lepton-plus-jets events using various methods in an attempt to select the jet corresponding to the true leptonic- $b$ parton the largest fraction of the time. During these studies numerous approaches were tried; for example: choosing the highest $E_{T}$ jet in the event, choosing the jet closest to the charged lepton, always choosing the tagged $b$ jet, using the jet matched to the leptonic- $b$ by 


\begin{tabular}{|c||c|}
\hline \hline Method & Corr. $b$ Selected \\
\hline Kinematic Fitter & $60-70 \%$ \\
Closest $b$-tagged jet to the lepton & $54 \%$ \\
Furthest $b$-tagged jet from the lepton & $49 \%$ \\
Closest of two highest- $E_{T}$ jets to the lepton & $35 \%$ \\
Closest jet to the lepton & $30 \%$ \\
Randomly selected jet & $25 \%$ \\
\hline \hline
\end{tabular}

Table 4.1: The results of various methods attepted to match jets to the correct $b$ parton. Percentages shown reflect the fraction of the time that each method matches a jet to the correct $b$-parton.

a kinematic fitter, or even choosing a jet at random. These studies demonstrated that using the jet assigned to be the leptonic- $b$ by a kinematic fitter ${ }^{1}$ was the best approach, as it correctly made this assignment the largest percentage of the time (Table 4.1).

In choosing a jet as the most probable match to the $b$ parton in the lepton-plusjets sample, each event is treated as a $t \bar{t}$ signal with top quark decays according to the standard model and kinematically reconstructed while constraining the top mass to $175 \mathrm{GeV} / \mathrm{c}^{2}$. The three-momenta of the charged lepton, two $b$ quarks and two light quarks are used in this reconstruction. Electron energy and muon momentum are measured with the calorimeter and tracking chamber. Information on $b$ and light quarks are taken from the four highest $E_{T}$ jets in the event. Jet energies are corrected for energy losses in cracks between calorimeter detectors and absolute hadron energy scale. Corrected jet energies are then required to be converted to the original parton energies (from top or $W$ decays) in order to reconstruct the constrained invariant mass of the top quark. These corrections were derived using a HERWIG $[62,63] t \bar{t}$ Monte Carlo sample, and depend on the parton type. The corrections, called the "topspecific corrections," have the primary purpose of unfolding the effect of the large jet energy resolution using the expected $p_{T}$ spectrum of jets from $t \bar{t}$ Monte Carlo,

\footnotetext{
${ }^{1}$ It is worthwhile to note that the kinematic fitter used by this analysis is exactly same as that used by CDF to make the top mass measurement.
} 
whereas the generic jet corrections assume a flat jet $p_{T}$ spectrum (see Figures 4.1 and 4.2). Additionally, by treating jets from $b$ quarks and light quarks separately, the top-specific corrections account for the different hadronization properties of the different jet types, and for the presence of semi-leptonic decays in $b$ jets, in which the energy of the neutrino is lost.

In $t \bar{t}$ lepton-plus-jets events with four high $E_{T}$ jets, there are $4 !=24$ possible permutations of jet-to-parton assignments. However, because jet ordering will not effect a calculation of the invariant mass of a hadronically-decaying $W$ (for example, the invariant mass of $W \rightarrow q \bar{q}^{\prime}$ will be the same for $q \bar{q}^{\prime}=$ jet $_{1}$ jet $_{2}$ or $q \bar{q}^{\prime}=$ jet $_{2}$ jet $\left._{1}\right)$ there are really only 12 permutations of jet assignments that merit consideration. For lepton-plus-jet events where one of the four $E_{T}$ jets is $b$-tagged the number of permutations is reduced again by a factor of two, because it does not make sense to assign a $b$-tagged jet to the partons from a $W$ boson which decayed hadronically $\left(W \rightarrow q \bar{q}^{\prime}\right.$ can include decays to $u, d, c$ or $s$ but rarely $\left.b\right)$. So, there are 6 ways to assign four leading jets to the four partons in single $b$-tagged lepton-plus-jets $t \bar{t}$ candidate events. Thus far we have ignored the missing energy aspect of leptonplus-jets events. Due to the ambiguity in the neutrino $p_{z}$, the number of kinematical combinations considered by the fitter for single-tagged events is $6 \times 2$.

The kinematic fitter assigns 5.0 and $0.5 \mathrm{GeV} / \mathrm{c}^{2}$ for the masses of the $b$ partons and the light partons, respectively (the charm quark, $c$, is treated as a light-quark by the kinematic fitter). In order to obtain the jet in the event which is most likely to be correctly matched to the $b$ parton from the semi-leptonic decay of the top-quark, the kinematic fitter constructs a $\chi^{2}$ for MINUIT [65] fitting as follows:

$$
\begin{aligned}
\chi^{2} & =\sum_{i=l, 4 \mathrm{jets}} \frac{\left(p_{T}^{i, \mathrm{fit}}-p_{T}^{i, \text { meas }}\right)^{2}}{\sigma_{i}^{2}}+\sum_{j=x, y} \frac{\left(p_{j}^{\mathrm{UE}, \mathrm{fit}}-p_{j}^{\mathrm{UE}, \mathrm{meas}}\right)^{2}}{\sigma_{j}^{2}} \\
& +\frac{\left(M_{j j}-M_{W}\right)^{2}}{\Gamma_{W}^{2}}+\frac{\left(M_{l \nu}-M_{W}\right)^{2}}{\Gamma_{W}^{2}}+\frac{\left(M_{b j j}-M_{t}\right)^{2}}{\Gamma_{t}^{2}}+\frac{\left(M_{b l \nu}-M_{t}\right)^{2}}{\Gamma_{t}^{2}}
\end{aligned}
$$

where $\sigma_{i=l}$ and $\sigma_{i=\text { jet }}$ correspond to the resolutions of the charged lepton and the 
four leading jets, $p_{j=x, y}^{\mathrm{UE}}$ and $\sigma_{j=x, y}$ correspond to the unclustered energy which is not clustered into jets. In this fit, the transverse energy of the neutrino is defined as the negative sum of the lepton, jet and unclustered energies. The two top quark masses are both constrained to be $175 \mathrm{GeV} / \mathrm{c}^{2}$ with a width $\Gamma_{t}=2.5$, and the two $W$ masses are also constrained to be the same with $M_{W}=80.41 \mathrm{GeV} / \mathrm{c}^{2}$ and $\Gamma_{W}=2.12$. In each event, the lowest $\chi^{2}$ from 12 combinations (or only 4 combinations in the case of double-tagged events) is selected as the best jet-to-parton assignment; this is referred to as $\chi_{\min }^{2}$. Optimization studies were carried out to determine if cutting on $\chi_{\min }^{2}$ would yield measurements with even higher precision, these studies are discussed in Section 4.3. As a result of the optimization studies, an additional requirement of $\chi_{\min }^{2}<20$ is found to give the best expected statistical uncertainty on the measured longitudinal fraction (the $\chi_{\min }^{2}$ cut is effective at rejecting badly reconstructed $t \bar{t}$ or background events). I find that a total of 31 candidates (out of 37 events in the lepton-plus-jets tagged dataset) pass the $\chi_{\min }^{2}$ cut. From Monte Carlo studies I have determined that this cut is $\sim 90 \%$ efficient for signal events and rejects $\sim 35 \%$ of the background.

\subsection{The Binned Likelihood Fitter}

A binned likelihood fit is used to extract the longitudinal fraction, $F_{0}$, from the data sample by comparing the $\cos \theta^{*}$ distribution for all $t \bar{t}$ candidate events to Monte Carlo templates for longitudinal and left-handed signal events, as well as a template for all background events.

\subsubsection{Acceptance Bias}

Due to the angular distribution in which the charged leptons from left-handed $W$ decays go preferentially backwards in the $W$ rest-frame (see Equations 1.9-1.11; the $p_{T}$ 

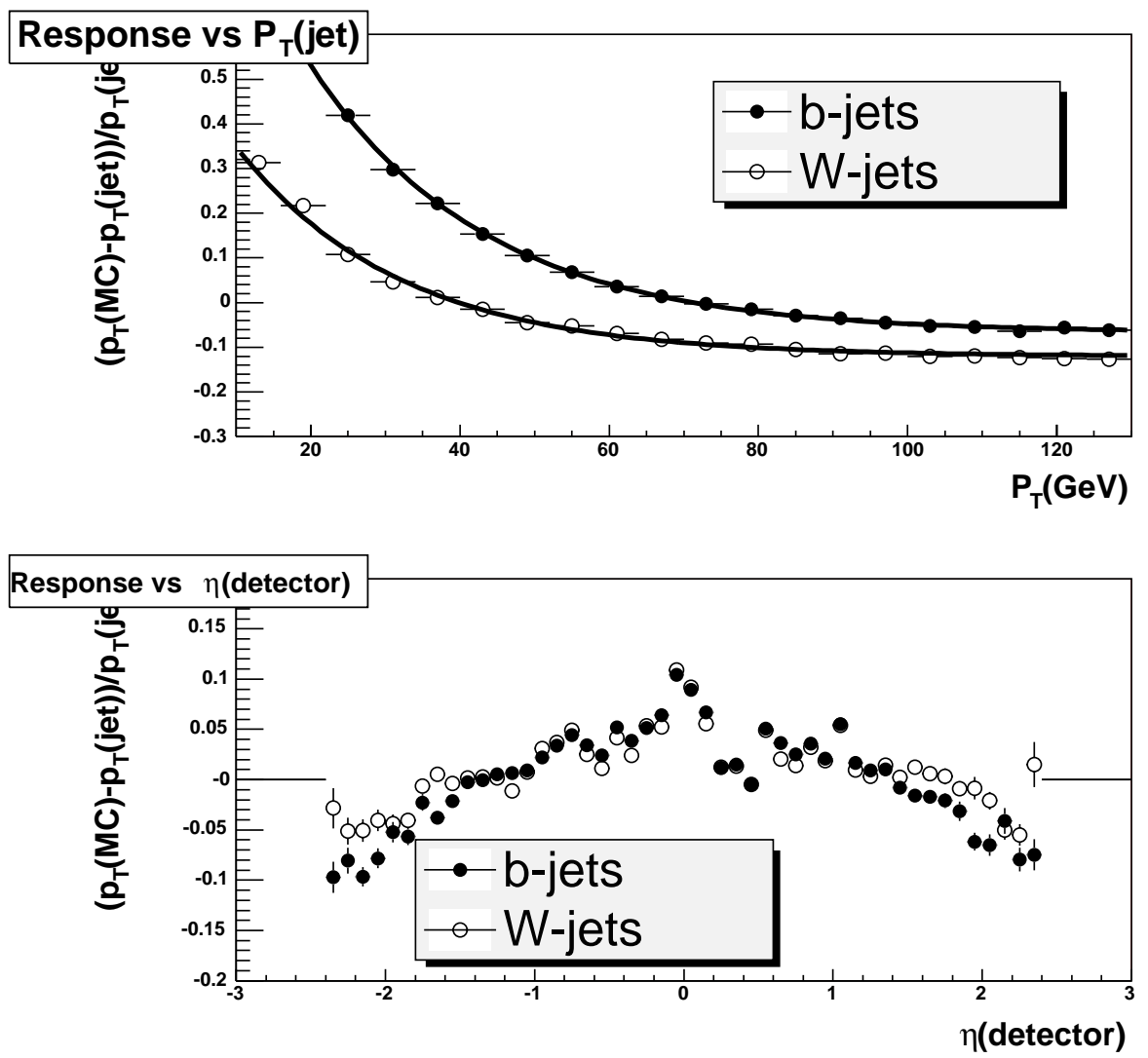

Figure 4.1: Top figure: The jet response, $\left(p_{T}^{\text {parton }}-p_{T}^{\text {jet }}\right) / p_{T}^{\text {jet }}$, as a function of $p_{T}^{\text {jet }}$ for $b$-jets and $W$-jets after only generic corrections are applied. Bottom figure: The jet response as a function of $\eta^{\text {jet }}$ after applying $t \bar{t}$-specific corrections that depend only on $p_{T}^{\text {jet }}$. Both of these figures are taken from [64], which details a measurement of the top quark mass at CDF during Run II. 

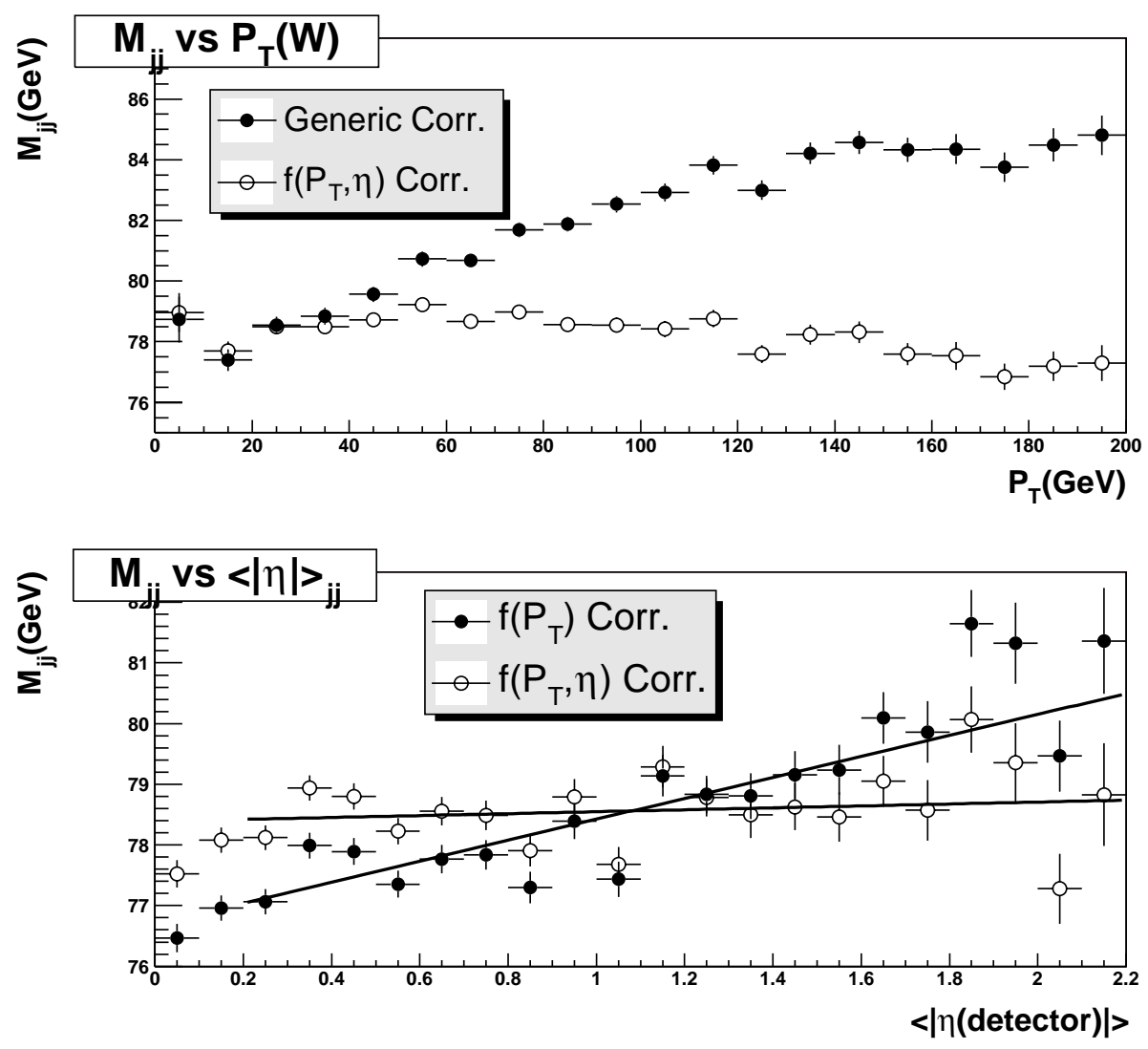

Figure 4.2: Top figure: The invariant mass of jets from $W$ daughters as a function of $p_{T}^{W}$ after applying generic corrections (full circles) and $p_{T}$ and $\eta$-dependent corrections (open circles). Bottom figure: The invariant mass of jets from $W$ daughters as a function of the average $\eta$ of $W$ jets after applying only $p_{T}$-dependent corrections (full circles) and $p_{T}$ and $\eta$ dependent corrections (open circles). In both figures, $\mathrm{MC}$ events with a good jet-parton match are used. Both of these figures are taken from [64], which details a measurement of the top quark mass at CDF during Run II. 
spectrum of the charged leptons is softer for left handed $W$ decay than for longitudinal $W$ decay, as depicted by Figure 1.11). This means that leptons which come from the decay of a longitudinally polarized $W$, for example, are more likely to pass the trigger thresholds and offline selection requirements than leptons from the decay of a lefthanded $W$. I need to correct for this acceptance bias in the binned likelihood fitter so that the true longitudinal fraction is measured. This derivation can be found in the Run II CDF lepton $p_{T}$ analysis [17], and to be consistent with that reference the same notation is used here.

The total number of left-handed, longitudinal and right-handed $W$ s which decay leptonically (here meant to denote $W \rightarrow e \nu$ and $W \rightarrow \mu \nu)$ in top decays can be expressed as:

$$
n=n_{L}+n_{0}+n_{R}
$$

where $n_{L}, n_{0}$ and $n_{R}$ are the number of left-handed, longitudinal and right-handed $W \mathrm{~s}$, respectively. The number of leptons actually observed by the CDF experiment are:

$$
n_{\text {obs }}=A_{L} n_{L}+A_{0} n_{0}+A_{R} n_{R}
$$

where $A_{L}, A_{0}$ and $A_{R}$ signify the individual fractions of leptons which pass the event selection and trigger requirements. The true and observed fractions of longitudinal $W$ s may be expresssed as:

$$
\begin{gathered}
F_{0}=\frac{n_{0}}{n} \\
F_{0}^{o b s}=\frac{A_{0} n_{0}}{n_{o b s}} .
\end{gathered}
$$

Similarly, one can write down the true and observed fractions of right-handed $W \mathrm{~s}$ :

$$
F_{R}=\frac{n_{R}}{n}
$$




$$
F_{R}^{o b s}=\frac{A_{R} n_{R}}{n_{o b s}}
$$

The observed longitudinal fraction, $F_{0}^{o b s}$, can then be written as a function of $F_{0}$ and $F_{R}$, the true longitudinal and right-handed fractions:

$$
F_{0}^{o b s}\left(F_{0}, F_{R}\right)=\frac{1}{1+\frac{A_{L}}{A_{0}}\left(\frac{1}{F_{0}}-1\right)+\frac{F_{R}}{F_{0}}\left(\frac{A_{R}}{A_{0}}-\frac{A_{L}}{A_{0}}\right)} .
$$

Since $F_{R}$, as predicted by the standard model, is expected to be very small (identically zero in the limit of a massless $b$ ), we set $F_{R}=0$ for this analysis. The expression for the observed longitudinal fraction in our fitter simplifies to:

$$
F_{0}^{o b s}\left(F_{0}\right)=\frac{1}{1+a_{L 0}\left(\frac{1}{F_{0}}-1\right)}
$$

where $a_{L 0}=A_{L} / A_{0}$ denotes the ratio of left-handed to longitudinal acceptances. Using the MadEvent signal Monte Carlo, I have measured this ratio of acceptance fractions to be $a_{L 0}=0.8327 \pm 0.0005$. Equation 4.9 serves to correct the bias on $F_{0}$ imposed by the event selection requirements. Recall that reconstructed chargedleptons are required to have $p_{T} \geq 20 \mathrm{GeV}$. Charged-leptons from left-handed $W$ bosons have a softer $p_{T}$ distribution than charged-leptons from longitudinal $W$ s. As a consequence, the $p_{T}$ requirement biases the data samples to have a higher average longitudinal fraction. The likelihood fitter uses Equation 4.9 for $F_{0}^{o b s}\left(F_{0}\right)$ when $F_{0} \in$ $[0,1]$, but when $F_{0} \notin[0,1]$ the $F_{0}^{o b s}\left(F_{0}\right)$ used in our prediction expression is simply $F_{0}$. This is done to avoid discontinuities in the correction terms. 


\subsubsection{The Likelihood Function}

To extract $F_{0}$ from the data, I use a binned maximum likelihood technique with the likelihood function defined as:

$$
\mathcal{L}=G\left(\beta ; \beta_{0}, \sigma\right) \prod_{i=1}^{N_{b}} \frac{\mu_{i}^{x_{i}} e^{-\mu_{i}}}{x_{i} !}
$$

where

$$
G\left(\beta ; \beta_{0}, \sigma\right)=\frac{1}{\sqrt{2 \pi \sigma^{2}}} \cdot e^{-\frac{\left(\beta-\beta_{0}\right)^{2}}{2 \sigma^{2}}}
$$

is a Gaussian constraint on $\beta$, the fraction of events due to background processes. The mean $\beta_{0}$ and the width $\sigma$ of the constraint describe an a priori estimate of the background content of the lepton-plus-jets sample. The product in Equation 4.10 is over all bins, where $N_{b}$ denotes the maximum number of bins. $\mu_{i}$ is the predicted content of bin $i$ and $x_{i}$ is the number of data events observed in bin $i$. The prediction, $\mu_{i}$, can be expressed as

$$
\mu_{i}=N_{e} \cdot\left(\beta T_{\mathrm{B}, i}+(1-\beta) F_{0}^{\mathrm{obs}}\left(F_{0}\right) T_{0, i}+(1-\beta)\left(1-F_{0}^{\mathrm{obs}}\left(F_{0}\right)\right) T_{\mathrm{L}, i}\right)
$$

where $T_{\mathrm{B}, i}$ denotes the content of the $i$ th bin for the background template; $T_{0, i}$ and $T_{\mathrm{L}, i}$ denote the bin content for the $i$ th bin of the longitudinal and left-handed templates, respectively.

\subsection{Kinematic Fitter Optimization Studies}

As mentioned earlier, I use a kinematic fitter as the optimal way of correctly choosing the "leptonic- $b$ " in an event. However, it is worthwhile to note that the top mass fitter operates in two modes (the free-fit mode where the two top quark masses in the event must be equal but are allowed to float, and constrained mode where again both 
top quark masses must be equal but are now fixed to a set value) and the sensitivity for measuring $F_{0}$ differs between them. Furthermore, it was noted that cutting on the variable ${ }^{2} \Delta m_{t}$ when using the kinematic fitter in the free-fit mode, or $\chi_{\min }^{2}$ for the constrained-fit mode has the potential to increase the precision of this measurement.

To determine which method and cut to use for the measurement, I carried out a series pseudo-experiments using the binned likelihood fitter of the form specified in Equation 4.10 with signal and background templates created from events generated by Monte Carlo programs. Measurements made during pseudo-experiments are conducted exactly like those on actual detector data, the only difference is that the events used to make the measurement during pseudo-experiments come from Monte Carlo event generator programs. In the case of pseudo-experiments conducted for these optimization studies, the "data" events are $\cos \theta^{*}$ values randomly selected from histograms of signal or background Monte Carlo events ${ }^{3}$ (Figures 5.1 and 5.4 in the next chapter depict these histograms). The total number of events in each pseudo-experiment, $N$, is selected from a Poisson distribution $P(N)=\frac{\mu^{N} e^{-\mu}}{N !}$, where $P(N)$ is the probability of observing a number $N$ and $\mu$ is the mean. The mean used for these studies is the number of events observed in the true lepton-plus-jets dataset with $\sim 162 \mathrm{pb}^{-1}$ (31 events). The fraction of background events is determined from the background estimate in Table 5.3 of the next chapter.

For each pseudo-experiment a measured value of $F_{0}$ is returned by the likelihood fitter. During the optimization studies, the measured $F_{0}$ values for a total of $10 \mathrm{k}$ pseudo-experiments were histogrammed together and fit to a Gaussian. During any given set of $10 \mathrm{k}$ pseudo-experiments the $\chi_{\min }^{2}$ cut or the $\Delta m_{t}$ cut would remain fixed. The width of the distribution fitted to a Gaussian is taken as the precision of the

\footnotetext{
${ }^{2} \Delta m_{t}=\left|m_{\text {top,fitter }}-m_{\text {top,PDG }}\right|$. In other words this variable is defined as the absolute difference between the PDG top mass, and the mass returned by the fitter in free-fit mode.

${ }^{3}$ This procedure is a bit like generating a $\sin \phi$ distribution in the $\phi$ interval $[0, \pi]$ by randomly choosing a $\phi_{0}$ value in this interval, and then keeping or rejecting the value depending on whether or not a second randomly chosen number between $[0,1]$ is $\leq \sin \phi_{0}$.
} 


\begin{tabular}{|c|c||c|c|}
\hline \multicolumn{2}{|c||}{ Free Fit } & \multicolumn{2}{c|}{ Constrained Fit } \\
\hline \hline$\Delta m_{t}$ cut $\left[\mathrm{GeV} / \mathrm{c}^{2}\right]$ & Uncertainty $F_{0}$ & $\chi_{\min }^{2}$ cut & Uncertainty $F_{0}$ \\
\hline$<\infty$ & \pm 0.34 & $<\infty$ & \pm 0.30 \\
$<100.0$ & \pm 0.34 & $<50.0$ & \pm 0.29 \\
$<75.0$ & \pm 0.34 & $<30.0$ & \pm 0.29 \\
$<60.0$ & \pm 0.33 & $<25.0$ & \pm 0.28 \\
$<50.0$ & \pm 0.31 & $<20.0$ & \pm 0.28 \\
$<40.0$ & \pm 0.30 & $<15.0$ & \pm 0.28 \\
$<30.0$ & \pm 0.30 & $<10.0$ & \pm 0.27 \\
$<20.0$ & \pm 0.30 & $<5.0$ & \pm 0.29 \\
$<15.0$ & \pm 0.31 & & \\
$<10.0$ & \pm 0.35 & & \\
$<5.0$ & - & \multicolumn{3}{|c}{} \\
\hline
\end{tabular}

Table 4.2: The results of pseudo-experiments carried out for optimization studies. The uncertainty on the uncertainties are not shown, but are about \pm 0.02 .

measured $F_{0}$ returned by the likelihood fitter for a set of pseudo-experiments carried out with that particular cut.

The results of these pseudo-experiments are shown in Table 4.2. For the measurement in the data one wants to use the method and cut which will yield the greatest precision. It is clear from this table that the constrained fit has a higher overall precision than the free fit. The table also reflects shallow minimums in the uncertainty values for the ranges of both cuts. To reach a decision about which method and cut to use for the measurement, I also examined the signal and background acceptances for each of the two methods (shown in Figure 4.3). I choose the constrained fit because it yields the highest overall sensitivity. I choose the $\chi_{\min }^{2}<20$ cut because of the higher acceptance of the signal events after making this cut $(\sim 0.90)$ and the relatively low acceptance of background events $(\sim 0.7)$.

\subsubsection{Verification of the Likelihood Technique}

To get a feeling for the sensitivity of measuring $F_{0}$ with the constrained fit and a $\chi_{\min }^{2}<20$ cut, I ran 10k pseudo-experiments where the standard model expectation 

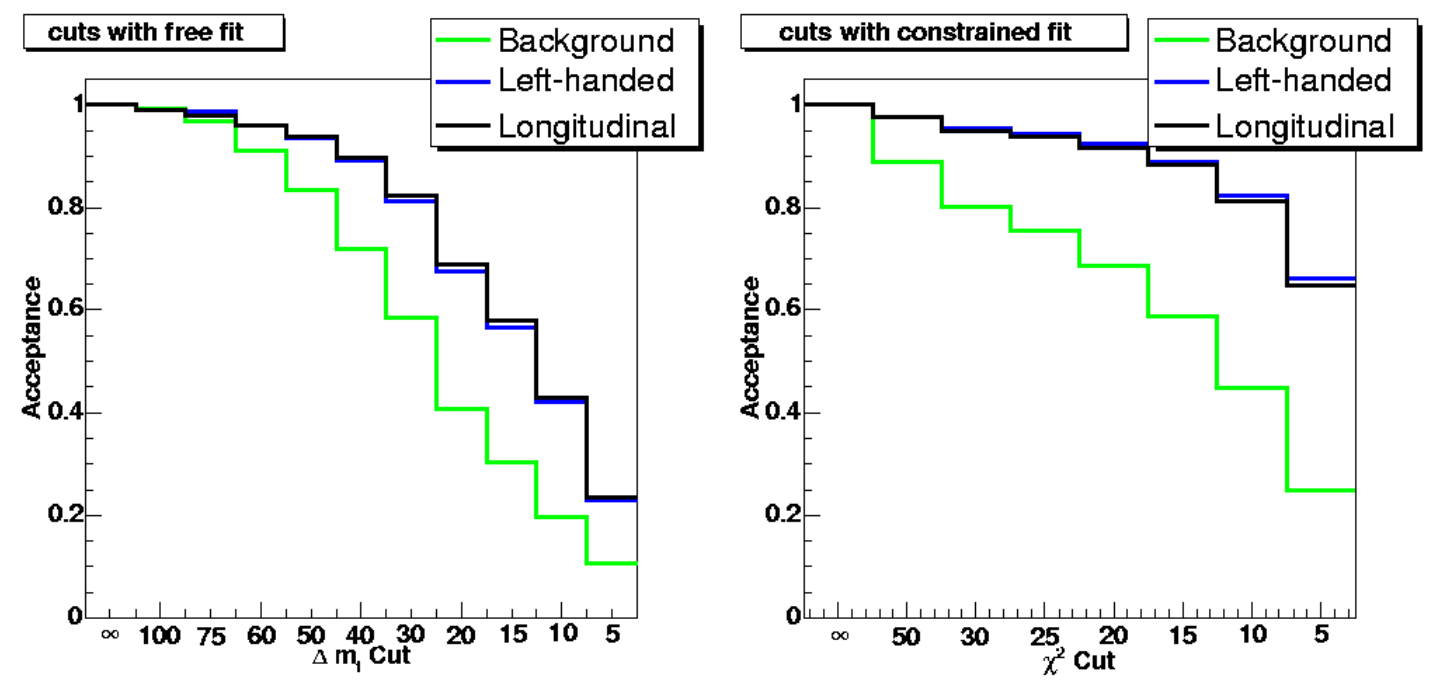

Figure 4.3: The results of signal and background acceptance studies for the free and the constrained fits from the top mass fitter.

for the fraction of longitudinal $W$ 's $\left(F_{0}=0.70\right)$ was used. Figure 4.4 shows the values of $F_{0}$ returned by the fitter for $10 \mathrm{k}$ pseudo-experiments using the $\chi_{\min }^{2}<20$ templates. Fitting a Gaussian to the distribution of measured $F_{0}$ values returns a mean measured value of $0.70 \pm 0.30$. The width of the Gaussian, 0.30, is our expected statistical uncertainty. The pull distribution from the same 10k pseudo-experiments was also examined and as Figure 4.5 shows, I obtain a pull distribution with a mean of $\sim 0$ and a $\sigma$ of $\sim 1$. This means that the likelihood fitter does not bias the measurement, and that the uncertainties returned by the fitter are accurate. Do note that the likelihood fitter does not restrict measurements to the physical region $F_{0} \in[0,1]$. How to interpret non-physical results which might be returned by the likelihood fitter is addressed in Chapter 8. 


\section{expt by expt measured F0}

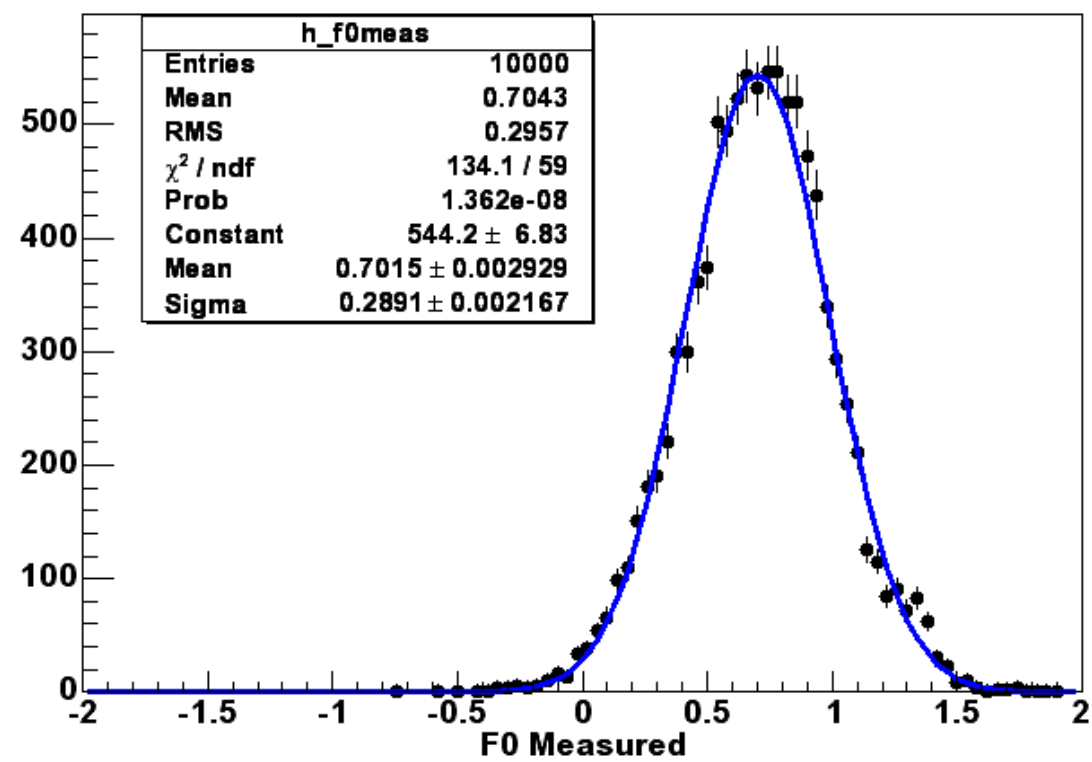

Figure 4.4: A Gaussian fit to the distribution of $F_{0}^{o b s}$ measured values for $10 \mathrm{k}$ pseudoexperiments where the constrained fit and a $\chi_{\min }^{2}<20$ cut were used.

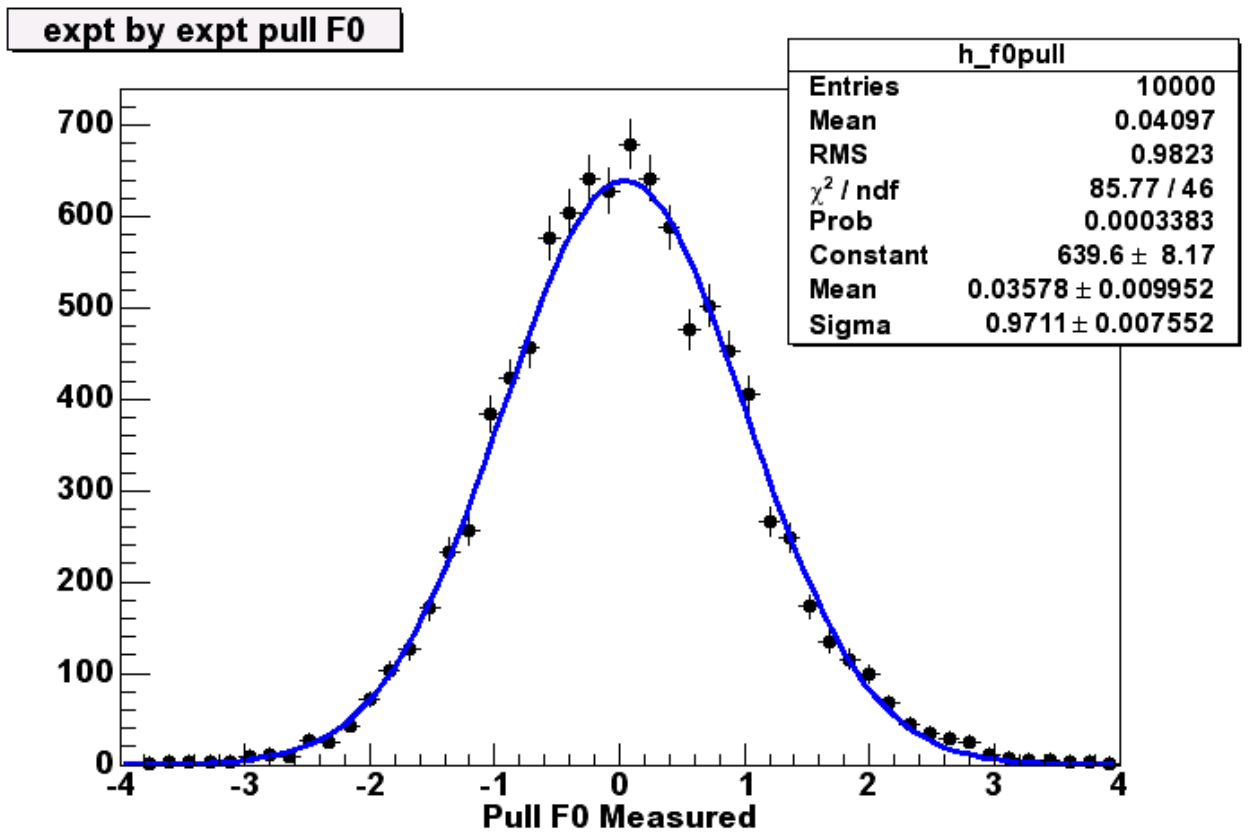

Figure 4.5: A Gaussian fit to the pull distribution for 10k pseudo-experiments where the constrained fit and a $\chi_{\min }^{2}<20$ cut were used. We define the pull as $\left(F_{0}^{\text {meas. }}\right.$ $\left.F_{0}^{a c t u a l}\right) / \delta F_{0}^{\text {meas. }}$. 


\section{Chapter 5}

\section{Models of Signal and Background}

A typical bunch crossing at the Tevatron results in the production of numerous particles traversing the CDF detector. An event signature, such as that for $t \bar{t}$ leptonplus-jets events is a single, isolated lepton, high $\mathbb{F}_{T}$ and numerous high- $p_{T}$ jets. It is difficult and extremely time consuming to calculate such interactions analytically. Instead, high-energy physicists rely on Monte Carlo ${ }^{1}$ programs for modeling signal and background processes. Small backgrounds from mis-identified secondary vertices, fake $W \mathrm{~s}$ and higher order processes such as $W b \bar{b}$ production are estimated a priori using Monte Carlo calculations as well as independent measurements in control data samples.

Generator-level Monte Carlo (MC) programs provide physicists with lists of particle four-vectors, however data from the experiment does not consist of nice clean four-vectors, and thus it is extremely difficult to make a successful direct comparison of generator-level MC to detector data. In order to make a comparison of theory to data, a detailed detector simulation program models the interactions of particles with the material inside the detector, as well as modeling the detector readout. This analysis uses several different event generators and complementary software to model signal and background events as well as their interactions with the CDF II detector.

\footnotetext{
${ }^{1}$ In physics, the term Monte Carlo is used to describe tools that are used to calculate an average by random sampling instead of sequencing through all theoretically possible combinations. The name is taken from a city famous for gambling-Monte Carlo, Monaco.
} 
Most of the measurements of acceptance and efficiency are carried out using PYTHIA v6.2 [66] or HERWIG v6.5 [62,63] Monte Carlo programs. Leading order matrix elements are employed by these generators for hard parton-level scattering processes, followed by parton showering to simulate gluon radiation and fragmentation. These generators are used in conjunction with the CTEQ5L parton distribution functions [67]. For accurate modeling of $b$ and $c$ hadron decays in the simulation of heavy-flavor jets, the decay algorithm QQ v9.1 [68] is used.

Special care is required to estimate the $b$-tagged backgrounds due to higher-order QCD processes such as $W b \bar{b}$ and $W c \bar{c}$. A study [44] of backgrounds in the $b$-tagged sample was conducted using the ALPGEN [69] leading-order matrix element Monte Carlo program to generate final states with multiple partons. These parton-level events are passed on to HERWIG and QQ for parton showering as well as $b$ and $c$ hadron decay.

The response of the CDF II detector to particles produced in $p \bar{p}$ collisions is reproduced by detector simulation. The detector geometry database used for simulation is identical to that used for offline event reconstruction. The GEANT3 [70] program is used by this offline software to track particles through various types and configurations of material. The deposition of charge in the silicon detectors is calculated using a simple geometric model based on the path length of the ionizing particle and a Landau distribution. The drift model for the COT tracker uses a parameterization of the GARFIELD [71] simulation tool where the parameters have been tuned to match the COT data [41]. Simulation of the calorimeter is done using the GFLASH [72] parameterization package interfaced with GEANT3. The GFLASH parameters are tuned to match the results of test beam data for electrons and high- $p_{T}$ pions. Additional detail on the CDF II detector simulation can be found elsewhere [73].

In all signal and background MC data samples, I apply the lepton-plus-jets event selection criteria, followed by kinematic reconstruction with a cut on the lowest $\chi^{2}$ fit 
as described in Chapter 4. The $\cos \theta^{*}$ distribution in each sample is obtained using the $m_{l b}$ approximation (Equation 1.13), where the invariant mass $m_{l b}$ is calculated on an event-by-event basis using the four-momenta of the charged-lepton and the jet assigned to the $b$-parton by the MINUIT [65] fitter.

The remainder of this chapter gives specifics about the generation of Monte Carlo to model the signal and background events, details of both the calculation of the various background contributions and the modeling of the $\cos \theta^{*}$ distributions for each of these contributing backgrounds.

\subsection{Models of Signal}

Several sets of $t \bar{t}$ signal Monte Carlo data were generated for use by this analysis. Signal MC data was generated to create the $\cos \theta^{*}$ distributions used as signal templates by the likelihood fitter, as well as $t \bar{t}$ signal MC samples created for systematics studies.

Signal MC data intended to model the left-handed and longitudinal $\cos \theta^{*}$ distributions used by the likelihood fitter were generated using a special fixed-helicity package spawned from leading-order MadEvent $[74,75] t \bar{t}$ Monte Carlo. ${ }^{2}$ MadEvent fixes the helicity of one $W$ boson to 0 or \pm 1 in the top rest-frame, while the helicity of the second $W$ takes on values predicted by the standard model (i.e., $\sim 70 \%$ longitudinal and $\sim 30 \%$ left-handed). Hadronization and fragmentation of the signal Monte Carlo events is carried out using PYTHIA. Additional details regarding the generation of these events can be found in Appendix D. The normalized signal templates used by this analysis are shown in Figure 5.1.

Additional $t \bar{t}$ MC data was generated for use in systematics studies. PYTHIA and HERWIG were both employed to generate this additional MC data. Some of these events were generated with different top masses or parton distribution functions.

\footnotetext{
${ }^{2}$ The effects of NLO contributions to the $W$ helicity in top decay are minimal $[76,77]$.
} 
Several samples have enhanced or reduced amounts of initial or final state radiation (see Chapter 7 for further details).

\subsection{Models of Backgrounds}

Backgrounds in the lepton-plus-jets channel are those processes which produce event signatures similar to the signal events: a single tight lepton, large $\mathbb{F}_{T}$, and multiple jets one or more of which is tagged as having a displaced secondary vertex. The non$t \bar{t}$ events in the lepton-plus-jets sample are from direct QCD production of heavyflavor quarks without an associated $W$ boson, higher-order production of a $W$ boson with heavy-flavor quarks, mistags of light quark jets in lepton-plus-jets events, and several other low-rate electroweak processes like single-top and diboson production. In this analysis, I need both the normalizations and the $\cos \theta^{*}$ shapes for each of these background contributions. The remainder of this chapter is devoted to describing the estimate of these backgrounds in detail.

\subsubsection{Non- $W$ QCD Backgrounds}

The non- $W$ QCD background is a mixture of events where an object identified in the detector as a single isolated lepton does not come from the decay of a $W$ (or $Z$ ) boson but occurs in an event with large missing energy. There are several ways that an isolated lepton can be faked in the detector: when part of a jet outside of the jet-cone passes the isolation requirement, when a photon conversion is misidentified as an electron, or when a pion with enough momentum to satisfy the muon $p_{T}$ selection criteria punches through the calorimeter arriving at the muon detectors to be misidentified. Real leptons in QCD background events arise from the semileptonic decay of $B$ hadrons. In these events, large missing energy can be faked when jets are mis-measured, when part of a jet deposits some fraction of its energy 

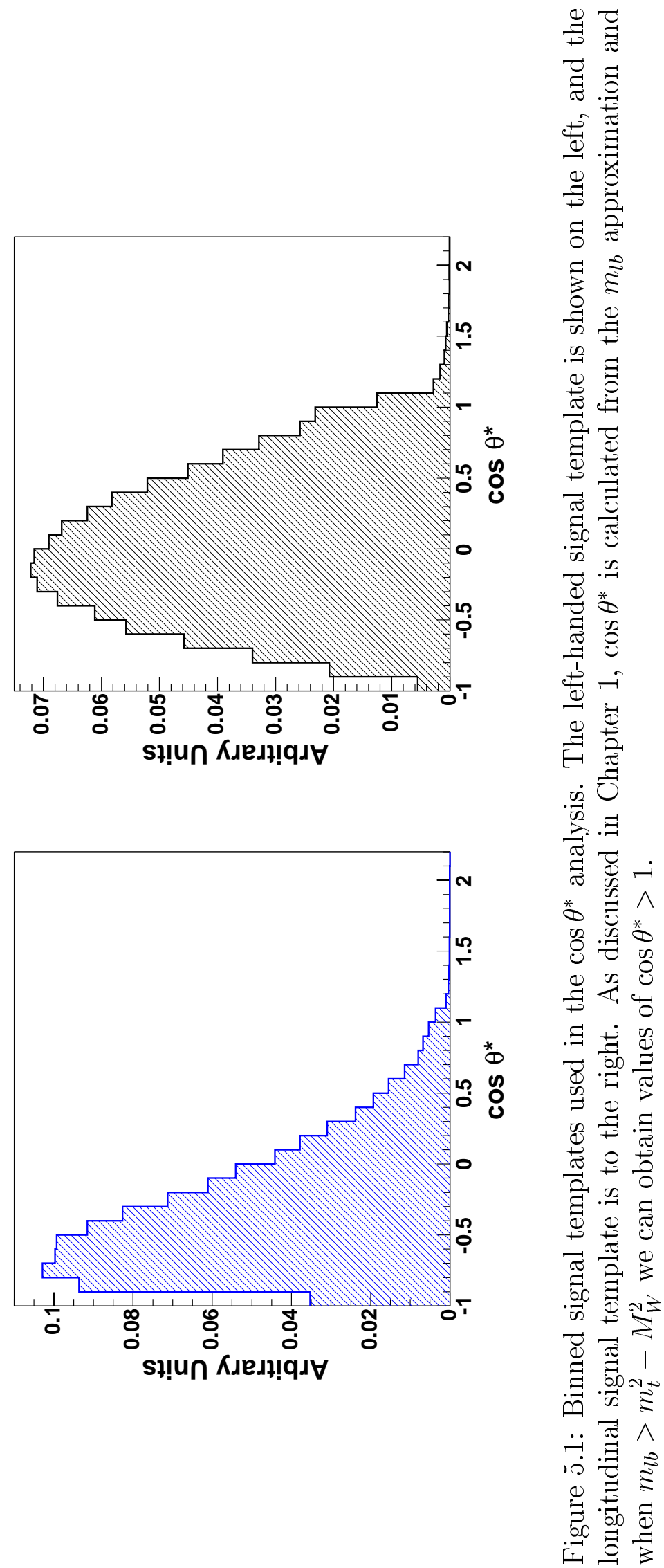
in a non-instrumented region of the calorimeter, or due to neutrinos in the case of heavy-flavor quark decays. Several backgrounds are calculated by normalizing to the total number of lepton-plus-jets events prior to $b$-tagging, so it is necessary to obtain an understanding for the amount of QCD background contamination in the pretag sample; some non- $W$ QCD background events may be $b$-tagged. The non- $W$ QCD background shape and normalization are taken from measurements of real detector data.

In events with leptonic decays of the $W$ boson, the charged lepton is isolated and there is large $\mathbb{F}_{T}$ due to the neutrino. In non- $W$ events this is not necessarily the case. The measurement of the non- $W$ QCD background exploits the assumption that the $\mathbb{F}_{T}$ and isolation of the tight lepton are uncorrelated in QCD events but highly correlated in $t \bar{t}$ events. In other words, the $\mathscr{F}_{T}$ spectra for isolated and non-isolated QCD background events look very similar. The non-isolated high- $p_{T}$ lepton events are an enriched sample of QCD events. We can define side-band regions in the $\mathscr{E}_{T^{-}}$ isolation plane to extrapolate the QCD expectations in the signal region (well isolated with large $\left.\mathscr{E}_{T}\right)$. We define four side-band regions in the $\mathscr{F}_{T}$-isolation plane:

- Region $A$ : isolation $>0.2$ and $\mathscr{t}_{T}<15 \mathrm{GeV}$

- Region $B$ : isolation $<0.1$ and $\mathscr{H}_{T}<15 \mathrm{GeV}$

- Region $C$ : isolation $>0.2$ and $\not_{T}>20 \mathrm{GeV}$

- Region $D$ : isolation $<0.1$ and $\mathbb{F}_{T}>20 \mathrm{GeV}$ ( $W$ signal region)

The ratio of non- $W$ events at low and high isolation in the low $\mathbb{F}_{T}$ region is assumed to be the same as in the high $\mathbb{F}_{T}$ region. Thus, the number of non- $W$ QCD events in the signal region can be estimated by:

$$
N_{D, \mathrm{QCD}}=N_{C} \times \frac{N_{B}}{N_{A}} .
$$


The contamination of true $W$ and $t \bar{t}$ events in the side-band regions is estimated using Monte Carlo samples.

Some of the non- $W$ QCD events are b-tagged and hence end up in the final event count. One estimate of this background applies Equation 5.1 to the sample of $b$ tagged events. Because this method is limited by the size of the tagged sample, the isolation boundary for regions $A$ and $C$ is lowered to the edge of the signal region (to isolation $>0.1$ ). The number of tagged events in the side-band region limits the precision on this estimate.

A second method used to estimate the non- $W$ QCD background scales the pretag QCD fraction by the average tagging rate for QCD events. The advantage of this approach is that it normalizes the background with the larger statistics available in the pretag sample, but utilizes a reliable estimate of the tag rate. The tagging rate in region $B$ for events with two or more jets is applied to the number of taggable jets in the signal region multiplied by the pretag non- $W$ QCD background fraction.

Both non- $W$ background estimates contribute to the weighted average shown in Table 5.1. The non- $W$ QCD shape used to model the contribution of these background events to the $\cos \theta^{*}$ distribution in the lepton-plus-jets sample comes from running over detector data with our usual lepton-plus-jets event selection criteria while reversing the isolation cut. This assumes that the $\cos \theta^{*}$ distribution for the non- $W$ background from isolated and non-isolated high $\not_{T}$ events are identical.

\subsubsection{Mistag Backgrounds}

The mistag background is comprised of lepton-plus-jets events where the jet tagged as having a SecVtx secondary vertex does not come from the decay of a heavy quark. For the mistag background we determine the number of events passing the selection criteria due to falsely tagging light-quark or gluonic jets. Detector simulation in Monte Carlo is capable of accurately reproducing the tag-rates for heavy-flavor jets, 
however not for the light-quark jets (which is known as the mistag rate). The mistag rate per jet is parameterized as a function of $\Sigma E_{T}^{j e t}$ of all jets in the event with $E_{T}>10 \mathrm{GeV}$ and $|\eta|<2.4$, the number of tracks, raw jet $E_{T}$, as well as jet $\eta$ and $\phi$. This parameterization is known as the mistag matrix. Additional details regarding the mistag matrix are discussed in Appendix E. The size of the mistag background is estimated by weighting each taggable jet with its mistag rate in the pretag sample. The mistag background estimate is shown in Table 5.1. The mistag matrix was run over $W$-plus-jets events generated from ALPGEN matrix element Monte Carlo pushed through HERWIG and the resulting shape was used to model the $\cos \theta^{*}$ distribution for this particular background.

\subsubsection{W plus Heavy Flavor Backgrounds}

A significant portion of the non-t $\bar{t}$ background for the tagged sample are processes where a $W$ boson is produced in association with heavy-flavor quarks. These processes are $W b \bar{b}, W c \bar{c}$ and $W c$ production.

To estimate these backgrounds, the event generator ALPGEN [69] is used to calculate exact matrix elements at leading order for a large set of parton-level processes described by QCD and the electroweak interactions. ALPGEN provides a proper treatment of heavy quark masses, spins and color flows. The heavy-flavor fractions, as calculated by ALPGEN, are calibrated against the fractions measured in inclusive jet data. Because these calculated fractions are observed to differ from those observed in jet data by as much as 50\%, they are renormalized [44]. These ALPGEN events are then pushed through HERWIG for hadronizaton and fragmentation.

The total contribution to the background from the $W$-plus-heavy-flavor processes is determined by multiplying the number of pretag lepton-plus-jets events in data by the measured $W$-plus-heavy-flavor fraction and the tagging efficiency observed in Monte Carlo. The heavy-flavor background estimate is shown in Table 5.1. 


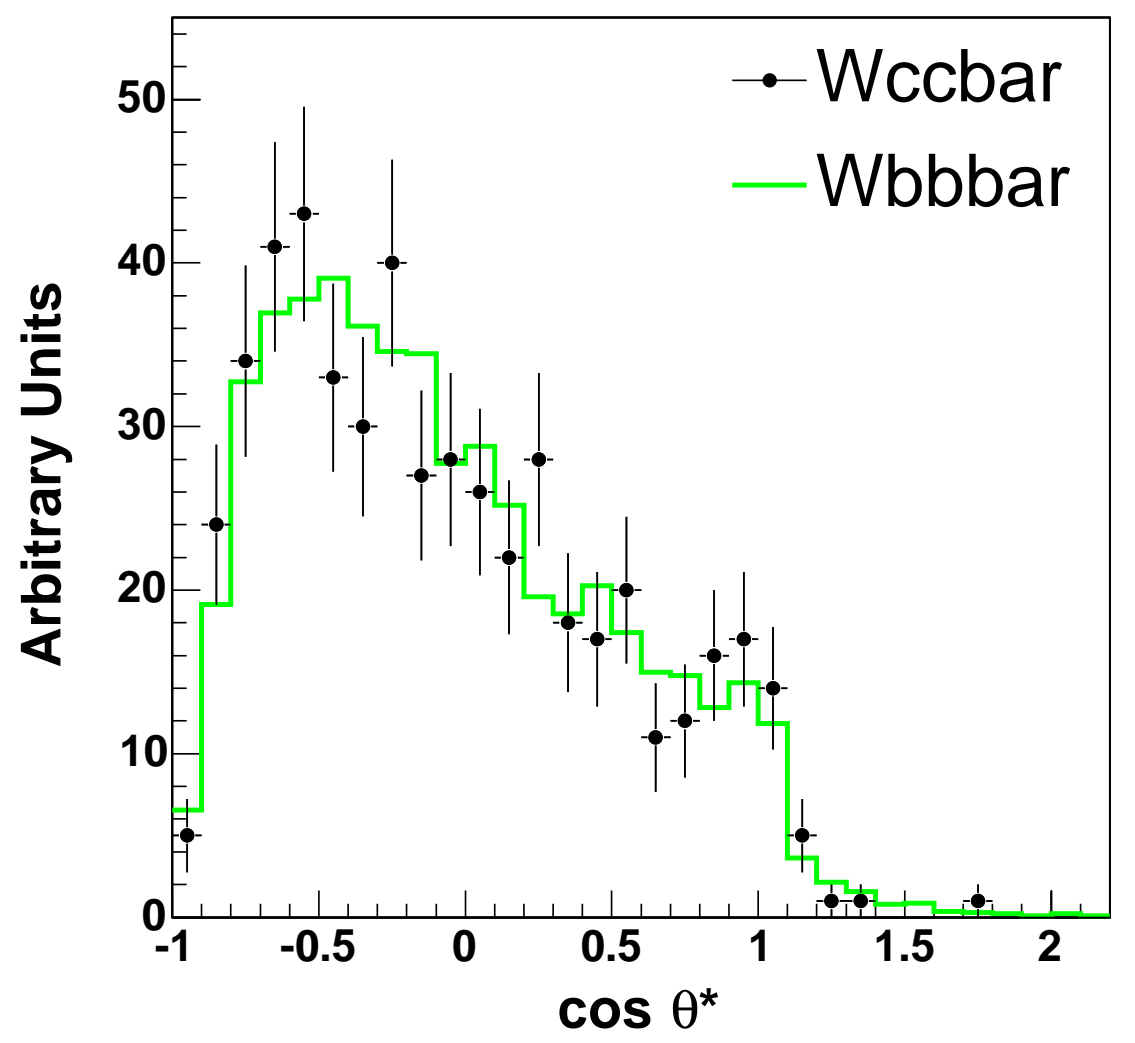

Figure 5.2: A comparison of the $W c \bar{c}$ and $W b \bar{b}$ shapes from Monte Carlo data. Due to this agreement, and the low statistics of the $W c \bar{c} \mathrm{MC}$ sample, the $W b \bar{b} \cos \theta^{*}$ distribution is used to model the $W c \bar{c}$ shape. 


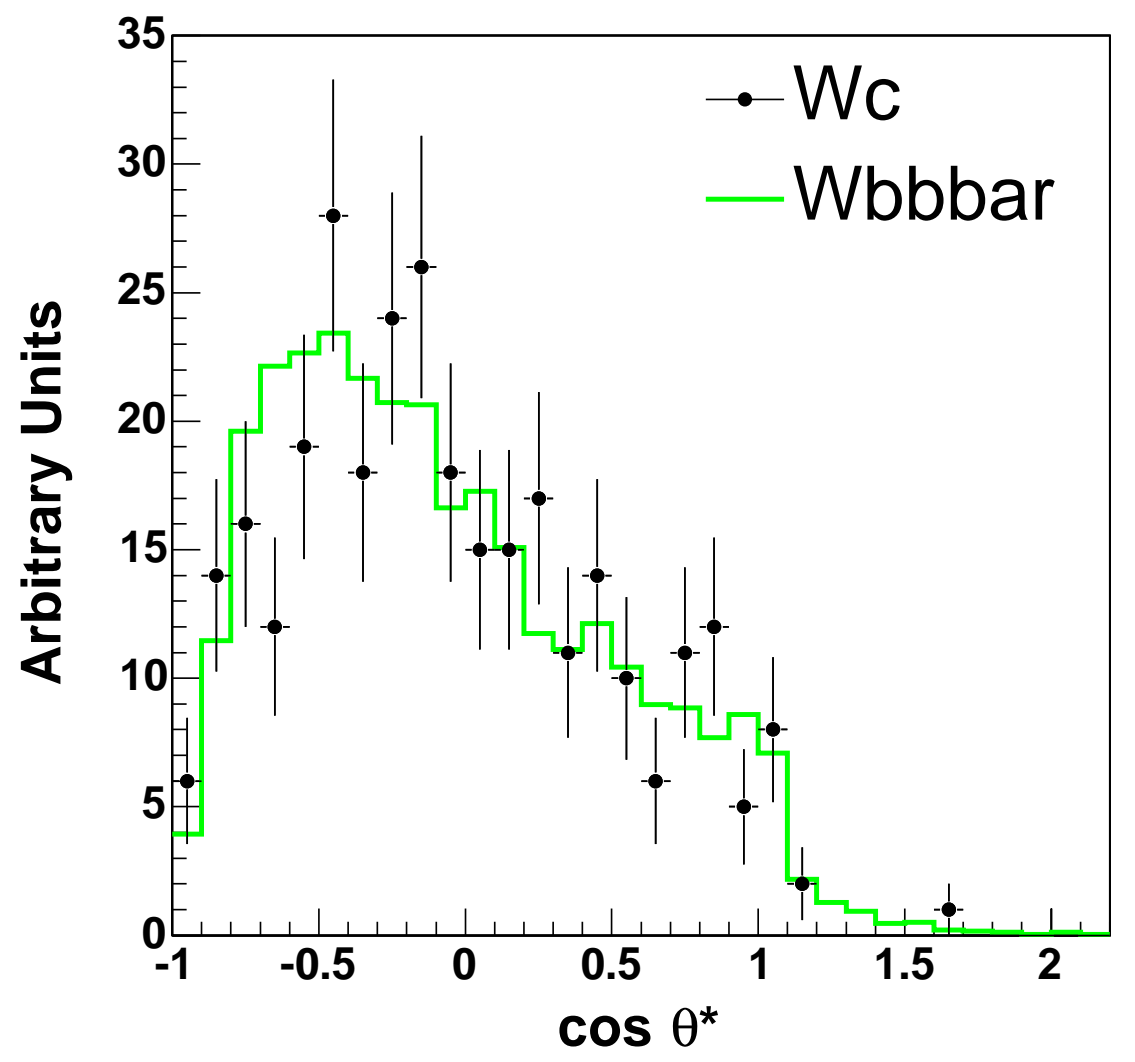

Figure 5.3: A comparison of the $W c$ and $W b \bar{b}$ shapes from Monte Carlo data. Due to this agreement, and the low statistics of the $W c \mathrm{MC}$ sample, the $W b \bar{b} \cos \theta^{*}$ distribution is used to model the $W c$ shape. 
The $\cos \theta^{*}$ distributions from $W b \bar{b}, W c \bar{c}$, and $W c$ events are all modeled with the $W b \bar{b}$ Monte Carlo events since, within the statistics available for the $W c \bar{c}$ and $W c$ processes, these shapes were observed to be in agreement with that from $W b \bar{b}$. Significantly larger MC statistics are available for $W b \bar{b}$ so I have used this shape to model the distributions for all three contributions. Plots showing the comparison of the $\cos \theta^{*}$ shape for the $W b \bar{b}, W c \bar{c}$, and $W c$ Monte Carlo events are shown in Figures 5.2 and 5.3.

\subsubsection{Electroweak Backgrounds}

Electroweak processes contribute six backgrounds to the lepton-plus-jets channel: $W W, W Z, Z Z, Z \rightarrow \tau \tau$, and the single-top $s$ and $t$-channel production modes. Calculation of electroweak backgrounds is done in a very straightforward manner. These electroweak diboson backgrounds are difficult to measure directly, due to the small value of their production cross-section multiplied by the branching ratios; the contribution of these backgrounds to the lepton-plus-jets sample is predicted using events from the ALPGEN matrix element Monte Carlo program pushed through HERWIG

for hadronization and fragmentation. The following formula is used to estimate the expected number of background events, $N_{\text {bkgnd }}$, for each of these six processes:

$$
N_{\text {bkgnd }}=\sigma \cdot B R \cdot S F \cdot\left(\sum_{\text {lepton }} \int \mathcal{L} d t \cdot \epsilon_{\text {accept }} \cdot \epsilon_{\text {tag }}\right)
$$

where $\sigma$ represents the NLO theoretical cross-section, $B R$ is the branching ratio for a given process, $S F$ is the $b$-tagging scale factor, $\int \mathcal{L} d t$ is the integrated luminosity, and $\epsilon_{\text {accept }}$ and $\epsilon_{\text {tag }}$ are the acceptance and tagging efficiencies, respectively. The sum is taken over all lepton types.

All of the diboson backgrounds, with accompanying jets can mimic the event signature of the signal $t \bar{t}$ lepton-plus-jets events. In the $W W$ background, one $W$ 
can decay leptonically and the other hadronically. For the $W Z$ background, the $W$ boson decays to a lepton-neutrino pair while the $Z$ boson decays into a heavy-flavor quark pair $(c \bar{c}$ or $b \bar{b})$. In the $Z Z$ background events, one of the $Z$ can bosons decay hadronically to charm or bottom while the other can decay leptonically. If one of the charged leptons from the $Z$ is undetected, it can give the appearance of large $\mathbb{E}_{T}$. In the process $Z \rightarrow \tau \tau$, the $t \bar{t}$ signal is mimicked when one $\tau$ decays hadronically and the other leptonically. The diboson and $Z \rightarrow \tau \tau$ background estimates are shown in Table 5.1.

Due to the very small contribution to the total background from the diboson and $Z \rightarrow \tau \tau$ processes, and the larger statistics available to model the $W b \bar{b}$ events, the $W b \bar{b}$ shape is used to model the $\cos \theta^{*}$ distribution for these backgrounds.

A single top quark can be produced through the electroweak interaction in association with a bottom quark. At the Tevatron, single-top can be produced through two mechanisms: the off-mass shell $W$ production ( $s$-channel), or through the $W$-gluon fusion process ( $t$-channel).

We model both the $s$ and $t$-channel production modes with PYTHIA Monte Carlo. We have sizable Monte Carlo samples for both $s$ and $t$-channel single-top events, so these samples are used directly to model the $\cos \theta^{*}$ distributions coming from these backgrounds. These background estimates are shown in Table 5.1.

\subsubsection{Background Summary}

In the tagged lepton-plus-jets sample, the requirement of at least one $b$-tag keeps the size of the backgrounds relatively small. the largest fraction of the background events come from QCD backgrounds where a jet has faked an electron or muon which passes the lepton isolation cut, and $W$ boson production associated with a heavy $b$-jet or gluon jets with a mis-identified $b$ ("mistags"). The expected total number

of background events is shown in Table 5.3 for the 3.5 -jet and 4-jet cases. The 


\begin{tabular}{|c||c|c|}
\hline \hline Background Source & 3.5 jets & $\geq 4$ jets \\
\hline Non- $W$ QCD & $1.7 \pm 0.5$ & $1.7 \pm 0.5$ \\
Mistags & $1.6 \pm 0.3$ & $1.2 \pm 0.3$ \\
$W b \bar{b}$ & $1.0 \pm 0.4$ & $0.6 \pm 0.3$ \\
$W c \bar{c}$ & $0.4 \pm 0.2$ & $0.2 \pm 0.1$ \\
$W c$ & $0.5 \pm 0.2$ & $0.2 \pm 0.1$ \\
$W W / W Z / Z Z / Z \rightarrow \tau \tau$ & $0.2 \pm 0.0$ & $0.1 \pm 0.0$ \\
Single top $(s$-channel $)$ & $0.18 \pm 0.03$ & $0.13 \pm 0.02$ \\
Single top $(t$-channel $)$ & $0.12 \pm 0.02$ & $0.06 \pm 0.02$ \\
\hline \hline
\end{tabular}

Table 5.1: Background estimates in the 3.5 and $\geq 4$-jet bins. These numbers do not include the acceptance factors due to the $\chi_{\min }^{2}<20$ cut (see Table 5.2).

background estimates of Table 5.3 take into account the event acceptance values for each of the backgrounds. The $\chi_{\min }^{2}<20$ cut on each of the backgrounds gives rise to several acceptance factors, determined for each background source from Monte Carlo events. These acceptance factors are shown in Table 5.2. Note that the acceptances for $W b \bar{b}, W c \bar{c}, W c$, diboson and $Z \rightarrow \tau \tau$ events have all been determined from $W b \bar{b}$ Monte Carlo events and are listed in Table 5.2 under the entry "Heavy Flavor + EWK".

The shape for the total background is taken from a histogram comprised of a sum of shapes where the individual distributions from the non- $W$ QCD, mistags, Heavy Flavor + Electroweak $($ EWK), $s$ and $t$-channel single top are merged together. The relative contributions of each background is taken from Table 5.3. The normalized background distribution is shown in Figure 5.4; this is the shape is used by the likelihood fitter as a template to model the background $\cos \theta^{*}$ distribution. 


\begin{tabular}{|c||c|c|}
\hline \hline Background Source & 3.5 jets & $\geq 4$ jets \\
\hline Non- $W$ QCD & $0.729 \pm 0.075$ & $0.775 \pm 0.098$ \\
Mistags & $0.683 \pm 0.071$ & $0.728 \pm 0.076$ \\
Heavy Flavor + EWK & $0.639 \pm 0.013$ & $0.660 \pm 0.015$ \\
Single top (s-channel) & $0.646 \pm 0.035$ & $0.673 \pm 0.042$ \\
Single top $(t$-channel $)$ & $0.784 \pm 0.077$ & $0.698 \pm 0.091$ \\
\hline \hline
\end{tabular}

Table 5.2: Acceptance factors in the 3.5 and $\geq 4$-jet bins. These numbers come from running the event selection criteria (including the $\chi_{\min }^{2}<20$ cut) on Monte Carlo events, or in the case of the non- $W$ QCD contribution, data. Note that the diboson and $Z \rightarrow \tau \tau$ acceptance numbers have been estimated using the heavy flavor Monte Carlo events (the table entry corresponding to "Heavy Flavor + EWK").

\begin{tabular}{|c||c|c|}
\hline \hline Background Source & 3.5 jets & $\geq 4$ jets \\
\hline Non- $W$ QCD & $1.2 \pm 0.4$ & $1.3 \pm 0.4$ \\
Mistags & $1.1 \pm 0.2$ & $0.9 \pm 0.2$ \\
Heavy Flavor + EWK & $1.3 \pm 0.5$ & $0.7 \pm 0.3$ \\
Single top ( $s$-channel) & $0.12 \pm 0.02$ & $0.09 \pm 0.01$ \\
Single top $(t$-channel $)$ & $0.09 \pm 0.02$ & $0.04 \pm 0.01$ \\
\hline Sub-total & $3.88 \pm 0.68$ & $3.05 \pm 0.59$ \\
\hline Total & \multicolumn{2}{|c|}{$6.93 \pm 0.90$} \\
\hline \hline
\end{tabular}

Table 5.3: Background estimates in the 3.5 and $\geq 4$-jet bins calculated using the pre-acceptance background estimates from Table 5.1 and the acceptance factor due to the $\chi_{\min }^{2}<20$ cut (Table 5.2). Note that the table entry for "Heavy Flavor + EWK" includes the background estimates for the diboson and $Z \rightarrow \tau \tau$ processes. For the 31 events which pass the selection criteria, the fraction of background events is determined to be $\beta_{0}=0.22 \pm 0.03$. 


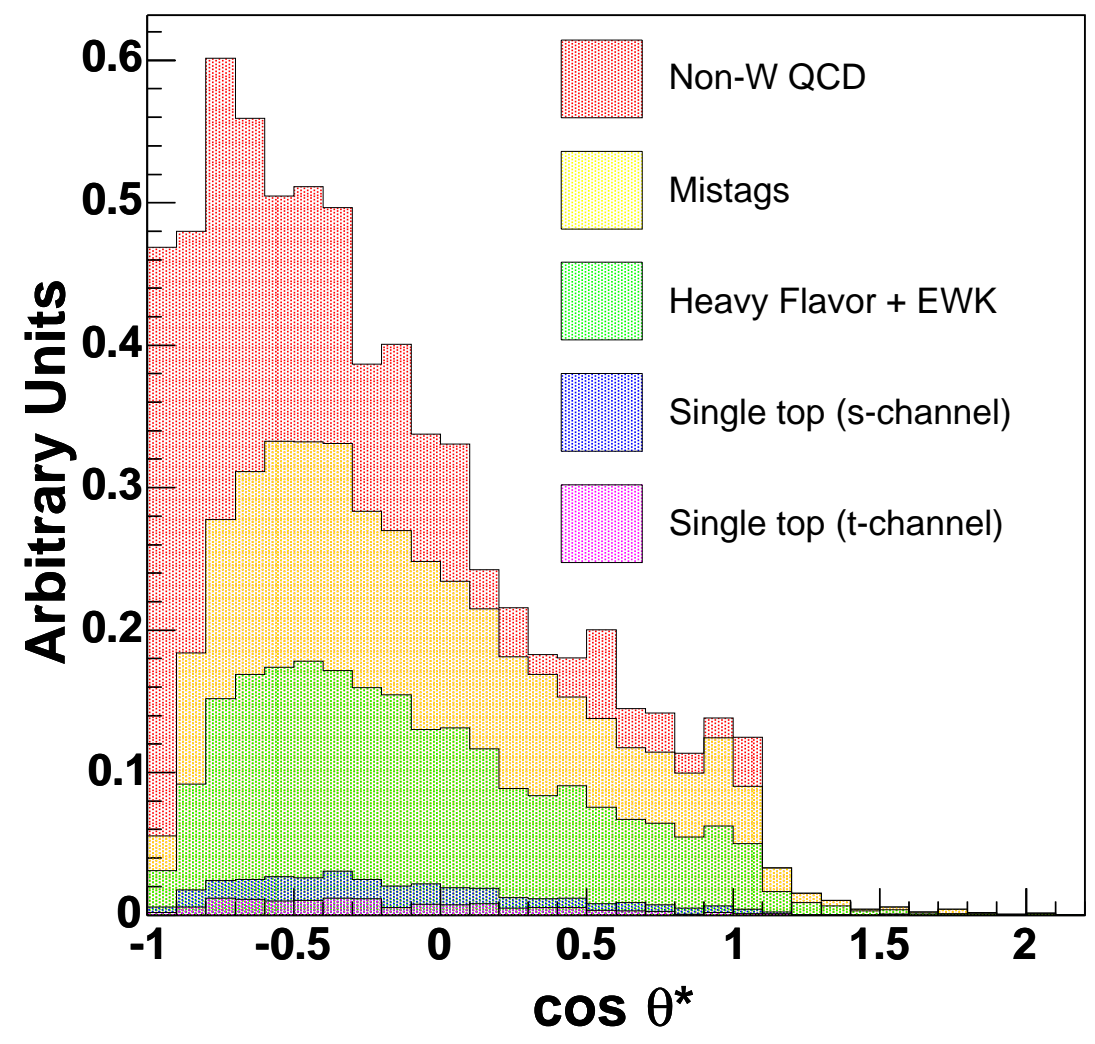

Figure 5.4: The sum of individual contributions to the background $\cos \theta^{*}$ template is obtained from data and Monte Carlo events. Data is used to model the non- $W$ QCD background and Monte Carlo simulation is used to model the Mistag, Heavy Flavor + EWK (modeled using $W b \bar{b}$ ), and single top backgrounds. The normalizations are taken from Table 5.3. 


\section{Chapter 6}

\section{Results}

A total of $31 t \bar{t}$ candidate events pass the event selection criteria in the $t \bar{t}$ lepton-plusjets dataset. Using the $m_{l b}$ approximation (Equation 1.13), and the leptonic $b$-jet as determined by the kinematic fitter, $\cos \theta^{*}$ is calculated for each of these 31 candidate events and a histogram showing the distribution of these values is constructed (Figure 6.1). The a priori estimate of the background fraction is $\beta_{0}=0.22 \pm 0.03$, as determined by the number of events in the background estimate of Table 5.3. A maximum likelihood fit to the $\cos \theta^{*}$ distribution associated with the $31 t \bar{t}$ events yields $F_{0}=0.99_{-0.35}^{+0.29}$ (stat.) for the fraction of longitudinally polarized $W$ bosons from top quark decays (the fit to the data events is also shown in Figure 6.1). The statistical uncertainty obtained is consistent with our expectations, as determined by running large numbers of pseudo-experiments (see Chapter 4). The positive asymmetric uncertainty on this measurement takes $F_{0}$ outside of the physically allowed region, $F_{0} \in[0,1]$; in Chapter 8 the method of Feldman-Cousins [78] is used to make a coherent statement about $F_{0}$ outside of the physical range. 


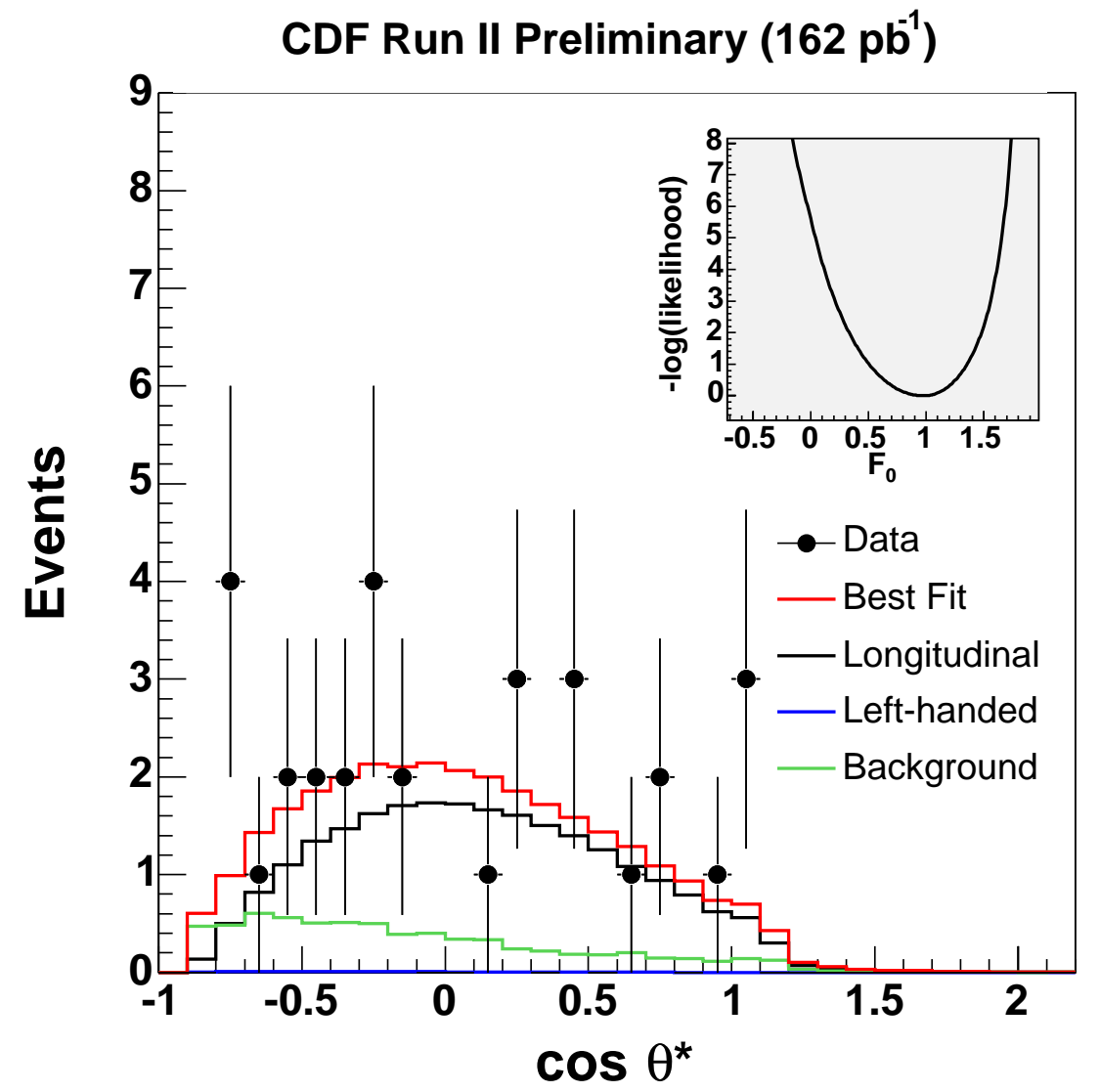

Figure 6.1: The $\cos \theta^{*}$ distribution for the lepton-plus-jets sample with signal and background templates normalized according to their Maximum Likelihood Estimators (MLEs) superimposed. Inset is the projection of negative log-likelihood along the $F_{0}$ axis for the fit to the data. As discussed in Chapter $1, \cos \theta^{*}$ is calculated from the $m_{l b}$ approximation and when $m_{l b}^{2}>m_{t}^{2}-M_{W}^{2}$ it is possible to obtain values of $\cos \theta^{*}>1$. Values of $\cos \theta^{*}<-1$ are not possible since $m_{l b}^{2} \nless 0$. 


\section{Chapter 7}

\section{Systematic Uncertainties}

Aside from the statistical uncertainty on the measurement of $F_{0}$, which arises due to the finite size of the data sample, the systematic uncertainties associated with a measurement of $F_{0}$ must also be considered. Systematic uncertainties can result from the calibration of the measurement apparatus (e.g., the jet energy scale), assumptions made by the experimenter by using a model with parameters which are not precisely known (e.g., the top mass), and the probability of detecting a type of decay or interaction (e.g., event acceptance bias).

In this analysis, all of the systematic uncertainties are determined by running large numbers of pseudo-experiments where each experiment is consistent with our expected sample size and background composition. For each systematic source, I ran multiple sets of such pseudo-experiments. More specifically, I generate data samples from signal and background Monte Carlo events where the total number of events in each experiment is allowed to fluctuate ${ }^{1}$ according to a Poisson distribution about a mean of 31 , the total number of events observed in the true data sample. The number of background events in each pseudo-experiment is also allowed to fluctuate, however these fluctuations are constrained to a Gaussian with a mean and width of 6.9 and 0.9 events, respectively (as determined by Table 5.3). These Monte Carlo events are reconstructed, I calculate $\cos \theta^{*}$, and fit the pseudo-experiment data using

\footnotetext{
${ }^{1}$ I generate a random integer $N$ according to a Poisson distribution $P(N)=\frac{\mu^{N} e^{-\mu}}{N !}$, where $P(N)$ is the probability of observing a number $N$ and $\mu$ is the mean.
} 
the procedure described in Chapter 4 . As is the case for determining many systematics in this analysis, the pseudo-data are generated from $\cos \theta^{*}$ distributions that I obtain after varying some particular aspect of our background or signal model by one standard deviation. I fit the resulting pseudo-data with the default signal and background templates and then compare this mean longitudinal fraction, $F_{0}$, to that measured using the default Monte Carlo.

I have considered the following sources of systematic uncertainty: The uncertainty in the background shape, the top mass uncertainty, the uncertainty due to the lack of understanding in the jet energy scale, uncertainties in the parton distribution functions, uncertainties in Monte Carlo program models, uncertainties in the amount of initial and final state radiation, uncertainties in the SecVtx $b$-tagging tagging efficiency and jet $E_{T}$ dependence, the finite Monte Carlo statistics used in signal template creation and the uncertainty in the acceptance correction. The reminder of this chapter discusses the contributions of each these to the total systematic uncertainty.

\subsection{Top Mass Uncertainty}

According to the standard model, the top quark and $W$ boson masses are the parameters which determines the fraction of longitudinal polarization in top decay (Equation 1.8). As discussed in Section 1.3, the mass of the top quark must be measured experimentally, as it is not predicted by the standard model. At the time that this analysis was carried out, the world average for the top mass was: $174.3 \pm 5.1$ $\mathrm{GeV} / \mathrm{c}^{2}[2]$. The $W$ boson mass is known with much greater precision than that of the top, $80.425 \pm 0.038 \mathrm{GeV} / \mathrm{c}^{2}[2]$, which means that the uncertainty in the longitudinal fraction due to the $W$ mass is negligible, however the uncertainty due to the top mass is not. The longitudinal fraction increases with increasing top mass (see Table 7.1). 


\begin{tabular}{|c|c|}
\hline \hline Top mass & Longitudinal Fraction \\
\hline 165 & 0.678 \\
170 & 0.691 \\
$174.3 \pm 5.1$ & $0.701 \pm 0.012$ \\
180 & 0.715 \\
185 & 0.726 \\
\hline \hline
\end{tabular}

Table 7.1: The fraction of longitudinal $W$ bosons for various top quark masses, as predicted by the standard model. The uncertainty in the longitudinal fraction for $m_{t}=174.3 \mathrm{GeV} / \mathrm{c}^{2}$ reflects the propagation of error on the top and $W$ masses. The $W$ mass used to obtain the numbers in the above table is $80.423 \pm 0.039 \mathrm{GeV} / \mathrm{c}^{2}$ [2].

The systematic uncertainty due to the top mass is estimated from the use of three sets of $10 \mathrm{k}$ pseudo-experiments where the number of events in each experiment is Poisson fluctuated about a mean of 31 events; one set for each of the masses: $m_{t}=170,175$, and $180 \mathrm{GeV} / \mathrm{c}^{2}$, which correspond to a shift of one standard deviation in either direction on the measured top quark mass. The HERWIG [62, 63] Monte Carlo program was used to generate $t \bar{t}$ events with these different top mass values, followed by full detector simulation. The background events for these pseudo-experiments are pulled from the default distribution (Figure 5.4). For each pseudo-experiment, I fit the $\cos \theta^{*}$ distribution obtained using the default signal and background templates in our fitter, and extract a measured $F_{0}$ value. After $10 \mathrm{k}$ pseudo-experiments, a distribution of $F_{0}$ values is obtained and fit to a Gaussian; the mean $F_{0}$ from this Gaussian fit is noted. After running one set of pseudo-experiments for each of the three mass values, the largest absolute difference between the mean $F_{0}$ for the shifted masses and the $F_{0}$ value measured at $m_{t}=175 \mathrm{GeV} / \mathrm{c}^{2}, 0.08$, is taken as the top mass systematic (see Table 7.4 at the end of this chapter). Figure 7.1 shows each of the Gaussian fits to these pseudo-data. 


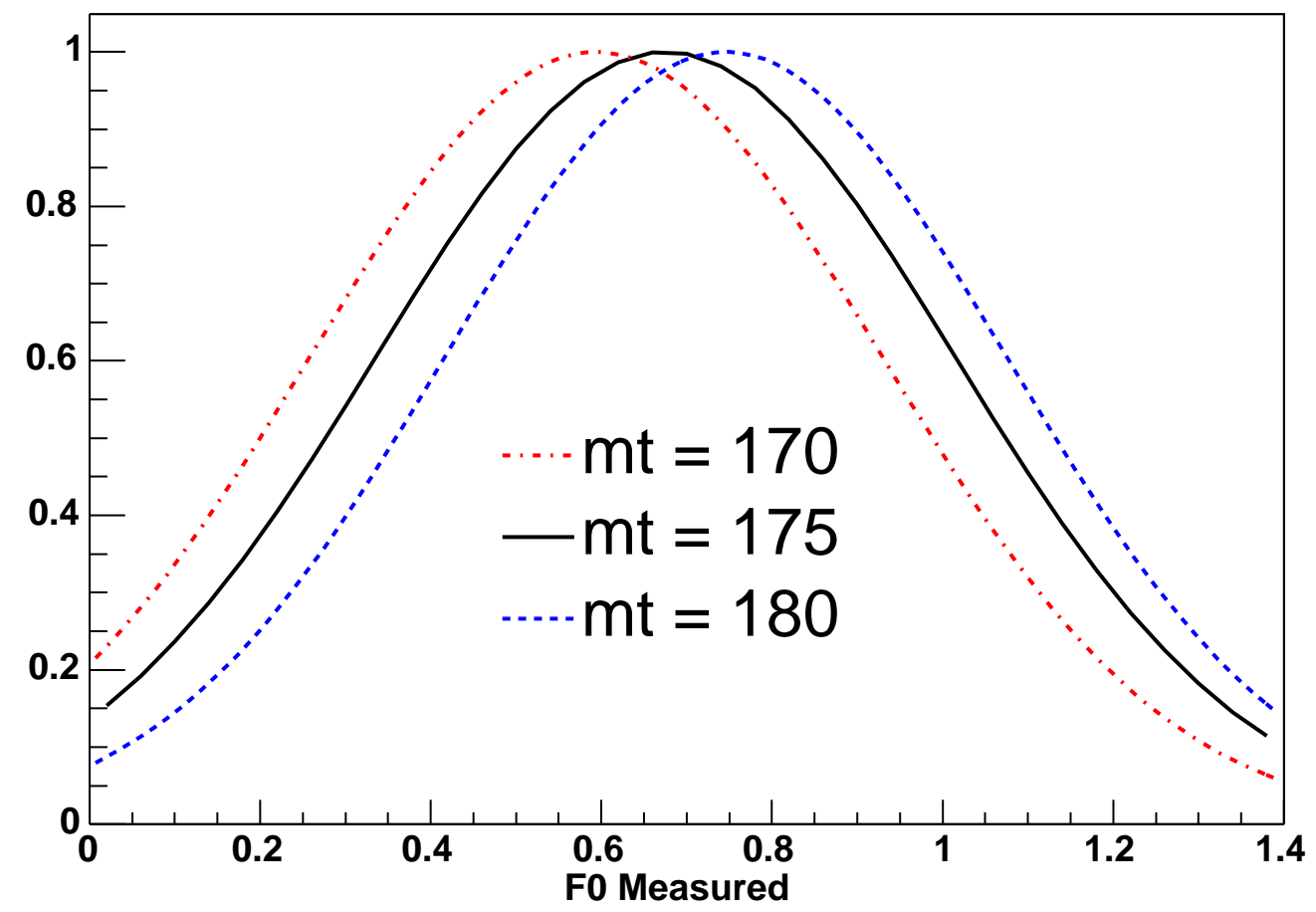

Figure 7.1: Gaussian fits to distributions of $10 \mathrm{k} F_{0}$ values obtained from the likelihood fitter by running pseudo-experiments. The black solid line shows the Gaussian fit to results from pseudo-experiments with $m_{t}=175 \mathrm{GeV} / \mathrm{c}^{2}$. The blue dashed line shows the Gaussian fit to results from pseudo-experiments with $m_{t}=180 \mathrm{GeV} / \mathrm{c}^{2}$. The red dash-dotted line shows the Gaussian fit to results from pseudo-experiments with $m_{t}=170 \mathrm{GeV} / \mathrm{c}^{2}$. The systematic uncertainty due to the top mass uncertainty is taken to be 0.08 .

\subsection{Jet Energy Scale}

This analysis calculates $\cos \theta^{*}$ using an approximation in terms of $m_{l b}$, the invariant mass of the lepton and the candidate $b$-jet. Since $m_{l b}^{2}=E_{b} E_{l}-\vec{p}_{b} \cdot \vec{p}_{l}$, the degree of accuracy to which we understand the jet energy scale has a direct effect on $m_{l b}$ and therefore the measurement of the longitudinal fraction.

The jet energy scale is used to scale the energy of the jet as measured by the detector to the final-state particle-level jet energy. There are also additional corrections made to associate the measured jet energy to the parent parton energy. At 
$\mathrm{CDF}$, the jet energy corrections are divided into several different levels, with each level accommodating different effects that can distort the measured jet energy. For example, the non-linear response of the calorimeter to different particle energies, or the effect of energy radiated by a jet lying outside of the cone defined by the clustering algorithm. Each of these jet corrections performed on the candidate $b$-jets has an associated uncertainty. If a particular correction modified the jet energy such that $E_{b}$

was over-corrected to be too high or low, the value of $m_{l b}^{2}$ would be shifted away from its true value-an effect which would translate into a corresponding shift in $\cos \theta^{*}$.

Our limit of understanding for the jet energy scale is represented by the associated systematic uncertainties. The purpose of these systematics is to account for differences between the actual jets observed in the detector data and how such jets are modeled in Monte Carlo. There are several contributions to the jet energy scale systematic uncertainty, each of these represents a lack of knowledge in the jet energy scale, and each of these can be a source of discrepancy between the modeling of jets in Monte Carlo and the actual jets observed in data:

\section{For data:}

- Data relative correction uncertainties

- Calorimeter stability over time

- Raw jet energy scale

- Multiple interaction uncertainties

\section{For Monte Carlo:}

- MC relative correction uncertainties

- MC raw jet scale

- Absolute scale 
- Out-of-Cone uncertainty for $0.4 \leq \Delta r \leq 1.0$

- Out-of-Cone "splash-out" for $\Delta r>1.0$

\section{Relative Corrections}

The relative corrections, applied to data and Monte Carlo events, are done to make the calorimeter response uniform as a function of $\eta$ by applying corrections derived from the di-jet balancing procedure [79]. This procedure exploits the property that the magnitude of the transverse momenta for two jets in two-jet event process should, in the absence of initial-state transverse momentum (where both jets are "back-toback"), be equal. This approach is known as "di-jet balancing" and is used to set the plug jet energy scale to the central scale. Jets are also corrected for any variation in response as a function of detector $\eta$.

Jets are best measured in the central region of the CDF detector with $0.2 \leq$ $|\eta| \leq 0.6$, as this region is away from detector cracks and is expected to have a stable response. This correction scales jets in the forward calorimeters to the central calorimeter scale since the central regions are better understood. I obtain a correction as a function of $\eta$ and $p_{T}$. The difference between data and Monte Carlo is taken as the systematic uncertainty for this correction.

\begin{tabular}{|c|c|c|}
\hline \hline Detector $\eta$ & Real Data & Simulated Data \\
\hline $0.0-0.1$ & $2 \%$ & $2 \%$ \\
$0.1-0.8$ & $0.2 \%$ & $0.2 \%$ \\
$0.8-1.4$ & $4 \%$ & $15 \%$ \\
$1.4-2.0$ & $4 \%$ & $4 \%$ \\
$>2.0$ & $7 \%$ & $7 \%$ \\
\hline \hline
\end{tabular}

Table 7.2: Uncertainties associated with relative corrections for various regions of detector $\eta[80]$. 


\section{Calorimeter Stability}

The gain of the plug calorimeter PMTs change as a function of time, mostly due to aging. By applying a correction for the calorimeter stability to detector, the data are corrected for this time variation.

\section{Raw Jet Energy Scale}

The raw jet energy scale is a factor applied to return the energy scale in the central calorimeter to that measured in the test beam for a single particle. This correction is different in real data and simulated events.

\section{Multiple Interactions}

When more than one $p \bar{p}$ pair interact in a given bunch-crossing, debris from additional collisions can fall into the jet cone, increasing the measured jet energy. From minimum bias data, where the CDF clock is used to trigger detector readout on bunch crossings instead of specific physics triggers, we determine the average energy deposited in a tower as a function of the number of reconstructed primary vertices and derive a correction to reverse the effect.

\section{Absolute Jet Energy Correction}

Since CDF calorimeters are non-linear, a correction must be applied. Here jets are corrected for fragmentation effects, calorimeter non-linearity as well as other effects like EM/HAD response and energy loss in un-instrumented regions of the detector. There are no high $E_{T}$ calibrations processes with a large number of statistics in the data, so this correction is extracted from Monte Carlo. 


\section{Underlying Event}

In a $p \bar{p}$ collision, part of the original proton and anti-proton momentum not involved in the hard parton interaction (i.e., the "spectator" partons) can flow into the calorimeter and increase the jet energies artificially. Depending on the needs of a particular analysis, this energy needs to be subtracted from the particle-level jet energy. This underlying event energy was measured from minimum bias data events where there is a requirement of exactly one reconstructed primary vertex imposed. This correction is derived from these events.

\section{Out-of-cone Correction}

Our goal is to determine the original parton energy from measurements of jet energy. This correction is applied to Monte Carlo data and is derived from simulated events. This correction adds back energy that, on average, falls outside of the cone used by the jet clustering algorithm.

\section{Jet Energy Scale Systematics}

To determine this systematic uncertainty, I apply a total $\operatorname{shift}^{2}( \pm \sigma)$ to jets in $t \bar{t}$ signal Monte Carlo, as well as all of our background samples. This method shifts the jet four-vectors and automatically sums in quadrature different contributions to the total jet energy systematic, depending on if the jet to be corrected is from Monte Carlo or true detector data.

After shifting the jet energies by one sigma in both the negative and positive directions, I run three sets of pseudo-experiments and fit the distributions of measured $F_{0}$ values obtained for each of these sets. The first set of $10 \mathrm{k}$ pseudo-experiments use the un-shifted HERWIG $t \bar{t}$ signal MC and the shapes obtained from the un-shifted

\footnotetext{
${ }^{2}$ The total shift on these samples is done via the setTotalSysUncertainties method of the Jet Corrections package $[80,81]$ in the CDF offline software.
} 
jet energies for all backgrounds. The un-shifted signal and background shapes are used to create distributions of "data" events which are then fit using the default likelihood fitter and templates. The other two sets of pseudo-experiments use the shapes from the shifted jet energies for the signal MC and backgrounds to create distributions of "data" events which are again fit using the likelihood fitter and the default templates. The resulting change in the mean $F_{0}$ measured during these sets of pseudo-experiments are summarized in Table 7.4. The systematic that I assign due to the uncertainty in the jet energy is taken to be the greatest absolute deviation from the un-shifted mean, 0.09 .

\subsection{Background Shape}

In pseudo-experiments conducted to determine the systematic uncertainty due to the background shape, the relative fractions of background events are enhanced or reduced.

As mentioned earlier, the background shape for this analysis is modeled using a linear combination of the non- $W$ QCD, mistag background, $W b \bar{b}$, and single-top shapes (see Chapter 5). The background template used in this analysis does not allow the individual contributions to the overall background to float-instead each contribution is fixed with a relative normalization as defined in Table 5.3. A shift in the contribution of one background relative to the others could result in a change in the overall background shape, thus affecting the measurement of the longitudinal polarization. A systematic uncertainty must be assigned for this effect. To determine this uncertainty I ran seven sets of 10k pseudo-experiments, where a different data distribution for each of the seven sets is created, each using the default signal template with a unique background shape. The unique shapes I investigated were those obtained exclusively from the $W b \bar{b}$, mistag and single-top Monte Carlo datasets as well as using the default 
background template but where the contribution from non- $W$ QCD background has been doubled or halved. One set of pseudo-experiments was carried out using the default background template. For each set of pseudo-experiments, I fit the distribution of $10 \mathrm{k} F_{0}$ values obtained to a Gaussian. I compare the means of these Gaussian fits for each of the seven sets of pseudo-experiments and take the largest deviation from the measured value when using the default background shape as the systematic uncertainty. The largest deviation I observe is 0.13 (see Figure 7.2 and Table 7.4), and this offset was attributed to the difference between using the default background shape and the background shape which was exclusively $W b \bar{b}$. This technique is conservative and over estimates the systematic uncertainty. In most cases, this method compares assumptions where the entire background shape is taken from one specific source or another whereas the real background shape in the data is created from a mixture of sources.

\subsection{Gluon Radiation}

A gluon emitted from a quark or another gluon is known as gluon radiation and comes in two varieties which can affect this measurement: A gluon which splits off before the $t \bar{t}$ pair is created, known as initial state radiation (ISR). Or, a gluon which splits off after the $t \bar{t}$ pair has been created, known as final state radiation (FSR).

ISR with gluons which are sufficiently hard has the potential of showing up as a high- $E_{T}$ jet in our candidate events. What is particularly problematic is that this extra jet is totally uncorrelated with the $t \bar{t}$ decay products and has the potential to degrade the accuracy of our event reconstruction, thus adversely affecting the $F_{0}$ measurement. On the other hand, hard FSR may carry away enough momentum and energy from a $b$-jet that the jet energy and direction are mis-measured as a consequence. 


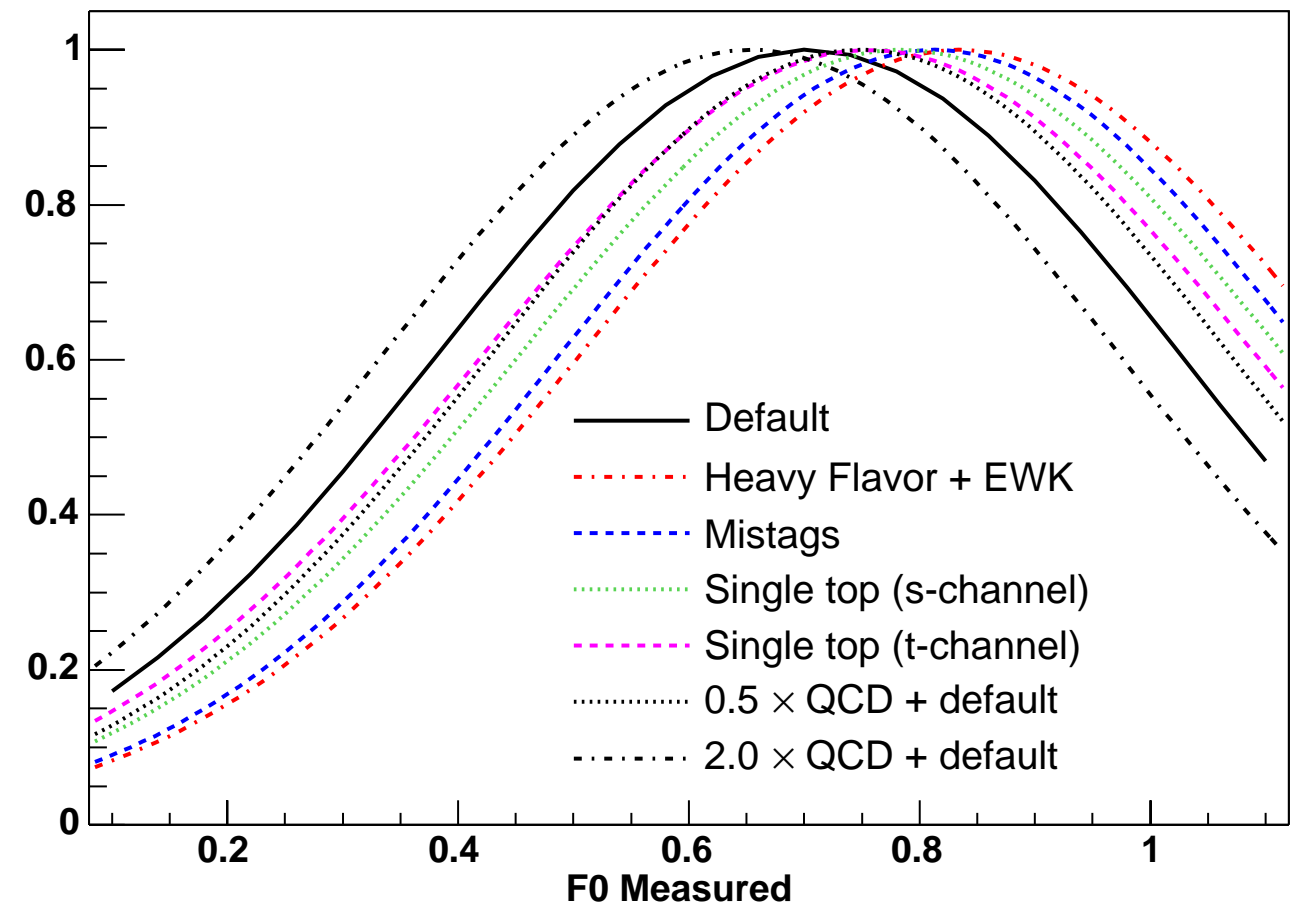

Figure 7.2: Gaussian fits to ditributions of $10 \mathrm{k} F_{0}$ values obtained from the likelihood fitter by running multiple sets of pseudo-experiments where the background shape is from one specific source (e.g., Single-top s-channel), or a particular combination of sources (e.g., the default shapes and normalizations but with twice the number of non- $W$ QCD events). I assign a systematic uncertainty due to the background shape equal to the absolute value of the largest deviation from the default mean, 0.13.

\subsubsection{Initial State Radiation}

To determine the systematic uncertainty due to uncertainties in the amount of ISR, I ran three sets of $10 \mathrm{k}$ pseudo-experiments where the signal shape that is used for generating the pseudo-experimental data is taken from a MC sample of PYTHIA "tune A" [82] $t \bar{t}$ events as well as events where ISR was enhanced or reduced. These samples of signal MC are used to model the $t \bar{t}$ events in our pseudo-experiments, and the background is modeled using the default template. I compare the mean $F_{0}$ measured by running the likelihood fitter with the default signal and background templates over each of these three samples, to the mean value obtained when running our fitter on 
a sample of $t \bar{t}$ events with the default amount of ISR. The difference in the mean $F_{0}$ values (see Table 7.4) were 0.010 and 0.019 for "less" and "more" ISR, respectively. I take the largest absolute deviation, 0.019, as the contribution to the systematic uncertainty due to initial state radiation.

\subsubsection{Final State Radiation}

To determine the systematic uncertainty due to FSR, I take the largest absolute difference in the mean $F_{0}$ measured for $10 \mathrm{k}$ pseudo-experiments where the signal distribution was obtained from the datasets where FSR was enhanced or reduced and the mean value measured for 10k pseudo-experiments with PYTHIA $t \bar{t}$ MC with the default amount of FSR. I use the default background template to generate background events. I find the largest absolute difference in the means to be 0.026 (see Table 7.4).

\subsection{Parton Distribution Functions}

I estimate the uncertainty due to using a particular parton distribution function (PDF) used to generate the signal templates. This measurement is susceptible to the PDF modeling uncertainties because different PDFs can affect event kinematics and therefore the $\cos \theta^{*}$ shape. To estimate this systematic uncertainty I ran three sets of $10 \mathrm{k}$ pseudo-experiments and compared the mean $F_{0}$ obtained from each of $t \bar{t}$ Monte Carlo samples using three different PDFs: CTEQ5L [67], MRST72 and MRST75 [83]. From these sets of pesudo-experiments (see Table 7.4), I determine the absolute mean difference between the default PDF CTEQ5L and the MRST72 PDF to be 0.029. Similarly, I find the absolute mean difference between the MRST72 and MRST75 PDFs to be 0.026. I sum both of these differences in quadrature to obtain the total PDF systematic of 0.04 . 


\subsection{Secondary Vertex Tagging}

\subsection{1 b-tagging Efficiency}

Studies that were carried out early in this analysis demonstrated that the top mass fitter assigns the correct jet as the leptonic- $b$ a larger fraction of the time for doubletagged events as compared to single-tagged events. Since tagging efficiency and the scale factor for b-tagging affects the single to double-tag ratio (and hence our template shapes), a systematic uncertainty due to SecVtx $b$-tagging must be assigned.

Several different sets of signal and background templates were created to evaluate this systematic. Instead of combining the single and double-tagged events into the same templates using the default single-to-double-tagged ratio, I created two sets of templates made exclusively from either single or double tagged events. With two sets of templates in hand, I combine the shapes obtained from single-tagged events with those from double-tagged events into templates which reflect several different single-to-double tagged ratios. The most reasonable ratios of single to double-tagged events were determined from plots of tag efficiencies (Figure 7.3) as a function of a parameter known to depend on them [84, 85]. The single to double-tag ratios I use in the pseudo-experiements are determined by reading off the tag efficiencies for both single and double-tagged events for four different values of this parameter $(b \Delta \epsilon=0.3,0.4,0.5$ and 0.6$)$. The templates created using the four different single-todouble tagged ratios were used to run $10 \mathrm{k}$ pseudo-experiments in the usual manner. I compared the mean measured $F_{0}$ from each of the four sets of pseudo-experiments to the mean $F_{0}$ measured from pseudo-experiments using the default templates (see Table 7.4). The largest absolute difference from the mean obtained using the default templates, 0.006, is taken to be the systematic uncertainty due to SecVtx $b$ tagging. 


\subsection{2 b-tagging Scale Factor}

For top analyses at CDF, the SecVtx $b$-tagging efficiency is assumed to be flat as a function of jet $E_{T}$ [44]. Although the $b$-jet $E_{T}$ distributions from the top decays to

longitudinal or transverse $W$ bosons is not different, I need to consider the effect of the $b$-tagging scale factor on a measurement of $F_{0}$.

This systematic is determined by fluctuating the data to Monte Carlo b-tagging scale factor, which is jet- $E_{T}$ dependent, within its uncertainties to weight events which go into the templates. I measure the shift in $F_{0}$ by running three sets of $10 \mathrm{k}$ pseudo-experiments each, always fitting the pseudo-data to the default templates. One set uses the default signal templates to generate the pseudo-data, and the other two sets use the weighted templates generated from a $\pm 1 \sigma$ shift in the $E_{T}$-dependent scale factor. Each of the $F_{0}$ distributions obtained using the shifted templates are fit to a Gaussian and the mean values are compared with the mean $F_{0}$ returned from pseudo-experiments that used the default templates. The largest deviation is 0.006 .

The two $b$-tagging systematics, due to the tagging efficiency and the scale factor, are summed in quadrature to obtain an overall b-tagging systematic of 0.006 (see Table 7.4).

\subsection{Monte Carlo Modeling}

In the process of carrying out $F_{0}$ measurements on pseudo-experiments using PYTHIA and HERWIG $t \bar{t}$ Monte Carlo to model "data" for systematics studies, it was noticed that the mean $F_{0}$ measured in the HERWIG and PYTHIA samples using our likelihood function with the default templates is not equal to $F_{0}=0.70$, which is what one expects to measure according to the standard model and $m_{t}=175 \mathrm{GeV} / \mathrm{c}^{2}$. Our signal templates are made from MadEvent MC pushed through PYTHIA for fragmentation and hadronization (See Appendix D). The largest absolute offset from the mean $F_{0}$ 
value I measure using MadEvent MC to model the signal in the pseudo-experiments is 0.04 (see Figure 7.4 and Table 7.4), therefore I assign this as the systematic due to Monte Carlo modeling.

\subsection{Acceptance Bias Correction}

Because there is an uncertainty on the acceptance correction factor, $a_{L 0}=0.8327 \pm$ 0.0005, the measurement of $F_{0}$ needs to include a systematic due to the fact the correction factor $a_{L 0}$ was determined from a finite number of signal Monte Carlo events. A shift in this acceptance factor could push the measured longitudinal fraction higher or lower than the true value in the data sample. To determine this systematic uncertainty, I run three sets of 10k pseudo-experiments on $t \bar{t}$ Monte Carlo using the default signal and background templates in the fitter. For each set of pseudoexperiments the value of $a_{L 0}$ in the likelihood function is set to $0.8327,0.8327+\sigma$, and $0.8327-\sigma$. The largest deviation in the mean $F_{0}$ measured is 0.0046 (see Table 7.4 ), so the systematic due to the uncertainty in the acceptance correction is taken as being $<0.005$.

\subsection{Monte Carlo Statistics}

A finite number of Monte Carlo signal events were used to generate our signal template shapes for the left-handed and the longitudinal distributions used by the likelihood fitter. I estimated the effect of using limited statistics for the signal templates on our measurement by Poisson fluctuating the contents of every bin in both the left-handed and longitudinal signal templates. I carry out 500 such fluctuations on our templates, running 1000 pseudo-experiments for each fluctuation. The mean $F_{0}$ measured for each set of 1000 pseudo-experiments is histogrammed separately, and the final distribution of 500 mean $F_{0}$ values is fit to a Gaussian. The sigma of this Gaussian fit, 
0.01, is taken to be the systematic due to the limited statistics used when generating our signal templates.

\subsection{Summary of Systematic Uncertainties}

The estimates for systematic uncertainties that have been considered are summarized in Table 7.3. The total systematic uncertainty, obtained by summing each variation in quadrature, is taken to be 0.19 .

\begin{tabular}{|c|c|}
\hline Systematic Source & $\overline{\Delta \Delta F_{0}}$ \\
\hline Background Shape & 0.13 \\
\hline Jet Energy Scale & 0.09 \\
\hline Top Mass Uncertainty & 0.08 \\
\hline PDFs & 0.04 \\
\hline MC Modeling & 0.04 \\
\hline ISR/FSR & 0.03 \\
\hline SecVtx $b$-tagging & 0.01 \\
\hline MC Statistics & 0.01 \\
\hline Acceptance Correction & $<0.005$ \\
\hline Total & 0.19 \\
\hline
\end{tabular}

Table 7.3: Systematics for the $W$ boson polarization $\cos \theta^{*}$ analysis. 


\begin{tabular}{|c|c|c|}
\hline$\overline{\text { Systematic }}$ & $\overline{\text { Dataset }}$ & $\overline{\overline{\Delta \Delta F_{0}}}$ \\
\hline Top Mass Uncertainty & $\begin{array}{l}m_{t}=175 \mathrm{GeV} / \mathrm{c}^{2} \\
m_{t}=170 \mathrm{GeV} / \mathrm{c}^{2} \\
m_{t}=180 \mathrm{GeV} / \mathrm{c}^{2}\end{array}$ & $\begin{array}{c}0.0 \text { (default) } \\
-0.071 \pm 0.003 \\
+0.081 \pm 0.003\end{array}$ \\
\hline Background Shape & $\begin{array}{c}\text { Default template } \\
\text { Heavy Flavor }+ \text { EWK } \\
\text { Mistags } \\
\text { Single top }(s \text {-channel }) \\
\text { Single top }(t \text {-channel }) \\
2.0 \times \text { QCD + Default } \\
0.5 \times \text { QCD + Default }\end{array}$ & $\begin{array}{c}0.0 \text { (default) } \\
+0.131 \pm 0.003 \\
+0.109 \pm 0.003 \\
+0.081 \pm 0.003 \\
+0.052 \pm 0.003 \\
+0.045 \pm 0.003 \\
-0.050 \pm 0.003\end{array}$ \\
\hline Jet Energy Scale & $\begin{array}{l}\text { no shift } \\
-\sigma \text { shift } \\
+\sigma \text { shift }\end{array}$ & $\begin{array}{c}0.0 \text { (default) } \\
+0.085 \pm 0.003 \\
+0.010 \pm 0.003\end{array}$ \\
\hline$\overline{\text { PDFs }^{\dagger}}$ & $\begin{array}{l}\text { |CTEQ5L-MRST72| } \\
\mid \text { MRST72 - MRST75 }\end{array}$ & $\begin{array}{l}0.029 \pm 0.003 \\
0.026 \pm 0.003\end{array}$ \\
\hline MC Modeling & $\begin{array}{c}\text { MadEvent }^{\ddagger} \\
\text { Herwig } \\
\text { Pythia }\end{array}$ & $\begin{array}{c}0.0 \text { (default) } \\
-0.028 \pm 0.003 \\
-0.039 \pm 0.003\end{array}$ \\
\hline$\overline{\text { ISR/FSR }}$ & $\begin{array}{c}\text { default amount } \\
\text { "less" ISR } \\
\text { "more" ISR } \\
\text { "less" FSR } \\
\text { "more" FSR }\end{array}$ & $\begin{array}{c}0.0 \text { (default) } \\
+0.010 \pm 0.003 \\
+0.019 \pm 0.003 \\
+0.006 \pm 0.003 \\
+0.026 \pm 0.003 \\
\end{array}$ \\
\hline $\bar{~} \overline{b \text {-tagging Efficincy }}$ & $\begin{array}{c}\text { default templates } \\
b \Delta \epsilon=0.3 \\
b \Delta \epsilon=0.4 \\
b \Delta \epsilon=0.5 \\
b \Delta \epsilon=0.6\end{array}$ & $\begin{array}{c}0.0 \text { (default) } \\
+0.0028 \pm 0.0031 \\
-0.0006 \pm 0.0032 \\
-0.0033 \pm 0.0032 \\
-0.0056 \pm 0.0032\end{array}$ \\
\hline$b$-tagging Scale Factor & $\begin{array}{c}\text { default templates } \\
-\sigma \text { shift } \\
+\sigma \text { shift }\end{array}$ & $\begin{array}{c}0.0 \text { (default) } \\
+0.0063 \pm 0.0032 \\
-0.0037 \pm 0.0032\end{array}$ \\
\hline Acceptance Correction & $\begin{array}{l}\text { no shift in } a_{L 0} \\
a_{L 0} \text { shifted }-\sigma \\
a_{L 0} \text { shifted }+\sigma\end{array}$ & $\begin{array}{c}0.0 \text { (default) } \\
-0.0046 \pm 0.0033 \\
+0.0029 \pm 0.0030\end{array}$ \\
\hline
\end{tabular}

Table 7.4: $\Delta F_{0}$ as determined by taking the difference between the mean $F_{0}$ measured with default shapes for 10k pseudo-experiments and that measured by shifting each systematic source by $\pm 1 \sigma .{ }^{\dagger}$ Numbers for the PDF $\Delta F_{0}$ denote the absolute difference. ${ }_{\ddagger}^{\ddagger}$ MadEvent was used for $t \bar{t}$ generation and PYTHIA was used for hadronization and fragmentation. 

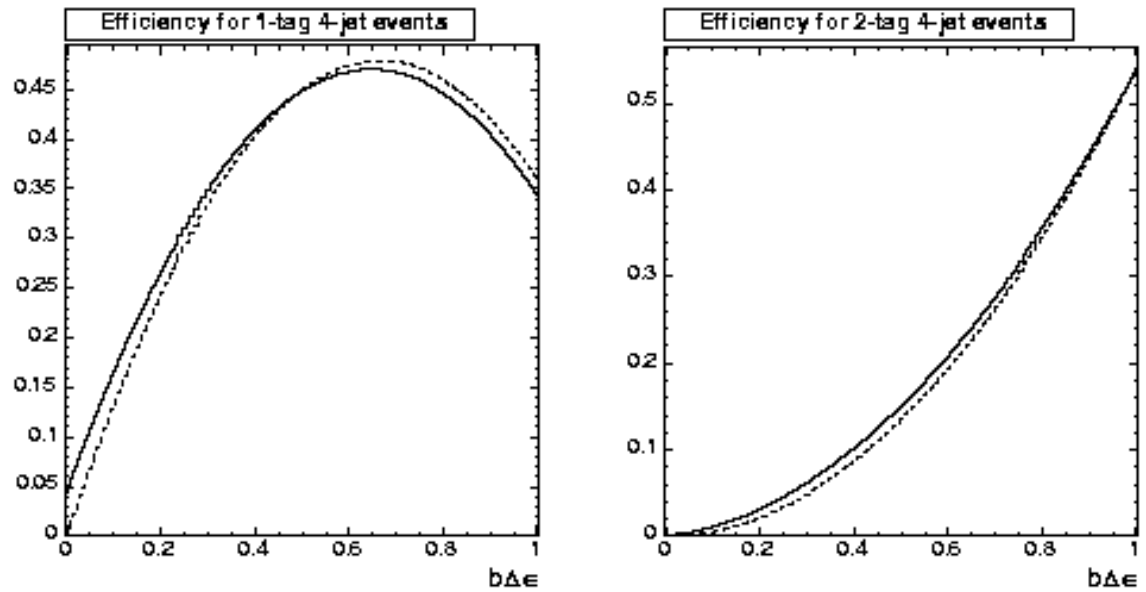

Figure 7.3: Plots taken from [84] which show tag efficiencies as a function of the parameter $b \Delta \epsilon$ for events reconstructed as lepton plus jets events with $\geq$ four jets. $b$ is the branching fraction for $t \rightarrow W b\left(\sim 1\right.$ in the standard model), and $\Delta \epsilon=\epsilon_{b}-\epsilon_{q}$ where $\epsilon_{q}$ is the probability of tagging a light quark [84, 85]. The solid lines denotes tag efficiencies as a function of $b \Delta \epsilon$, the dashed lines denote tag efficiencies as a function of $b \Delta \epsilon$ assuming that $\epsilon_{c}=\epsilon_{q}=0$. 


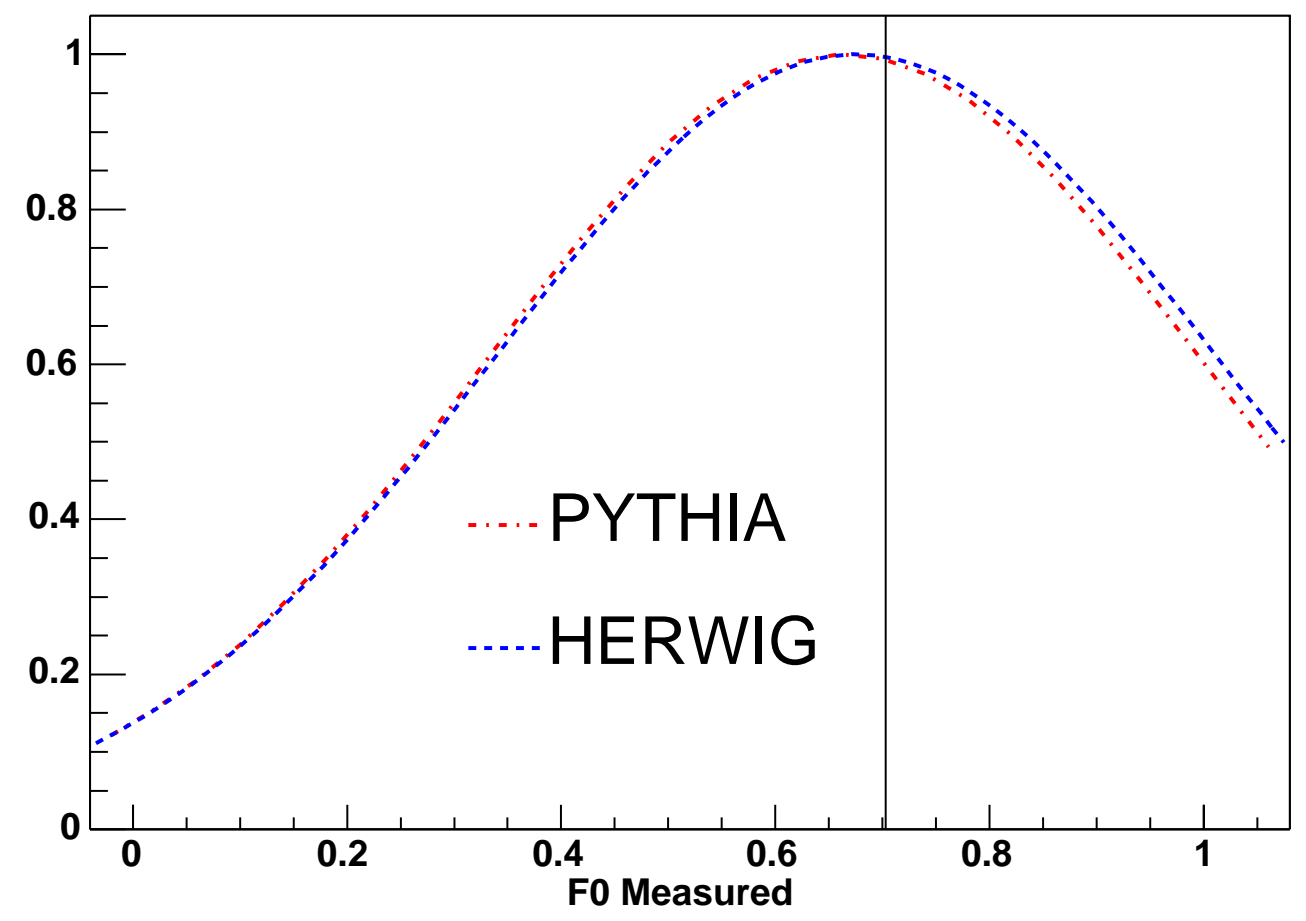

Figure 7.4: Gaussian fits to distributions of $10 \mathrm{k} F_{0}$ values obtained from the likelihood fitter by running pseudo-experiments where the signal is modeled by PYTHIA (red dash-dotted line) or HERWIG (blue dashed line) Monte Carlo programs. The black vertical line shows the mean value from a Gaussian fit to results from pseudo-experiments using MadEvent Monte Carlo to model the signal where $F_{0}=0.70$. Because of this shift, I assign a systematic uncertainty due to Monte Carlo Modeling equal to the absolute value of the largest deviation from the vertical line, 0.04 . 


\section{Chapter 8}

\section{Confidence Interval Estimation}

The estimate $F_{0}$ is taken to be the value $\hat{F}_{0}$ which maximizes the likelihood function; this value is called the Maximum Likelihood Estimate (MLE). The true longitudinal fraction, $F_{0}$, is defined in the physically allowed interval $F_{0} \in[0,1]$. However, as a parameter of the likelihood function (Equation 4.10) $F_{0}$ is not restricted to this range. So that a coherent statement about $F_{0}$, given all possible measurement outcomes, can be made the method of Feldman and Cousins $[78,17]$ is used. This method always produces confidence intervals within the defined range, allowing one to avoid quoting confidence-intervals outside of the physically-allowed region.

To construct confidence intervals using the Feldman-Cousins method, one must first gain an understanding of experimental resolution and bias for all possible values of $F_{0}$. It is possible to gain such understanding by running many sets of pseudoexperiments, each consistent with the lepton-plus-jets sample size and background composition. During these sets of pseudo-experiments the true parameter $F_{0}$ is allowed to vary uniformly from set to set between 0 and 1 and events are generated accordingly. The pseudo-experiment data are fit according to the measurement pro-

cedure described in Chapter 4 to obtain the MLE, $\hat{F}_{0}$, for each pseudo-experiment. The distribution of MLEs for constant $F_{0}$ values was observed to be Gaussian. Parameterizations of the mean and width of the distribution of MLEs are constructed as a function of the true parameter, i.e., $\mu\left(F_{0}\right)$ and $\sigma\left(F_{0}\right)$ (see Figures 8.2 and 8.3). 
These functions are assembled to form the resolution function

$$
P\left(\hat{F}_{0} ; F_{0}\right)=G\left(\hat{F} ; \mu\left(F_{0}\right), \sigma\left(F_{0}\right)\right)
$$

The resolution function is modified to incorporate systematic uncertainties into the Feldman-Cousins method by adding the estimate of the overall systematic uncertainty, $\sigma_{\text {syst. }}$, in quadrature with the statistical uncertainty, $\sigma\left(F_{0}\right)$ :

$$
P\left(\hat{F}_{0} ; F_{0}\right)=G\left(\hat{F}_{0} ; \mu\left(F_{0}\right), \sqrt{\sigma^{2}\left(F_{0}\right)+\sigma_{\text {syst. }}^{2}}\right) .
$$

Feldman-Cousins confidence intervals are constructed at the $68.3 \%, 95.4 \%$ and 99.7\% confidence levels. These intervals are shown in Figure 8.1, along with the MLE for this measurement. From these confidence intervals and the MLE at $\hat{F}_{0}=0.99$, we find $F_{0}>0.33 @ 95 \% \mathrm{CL}$. 


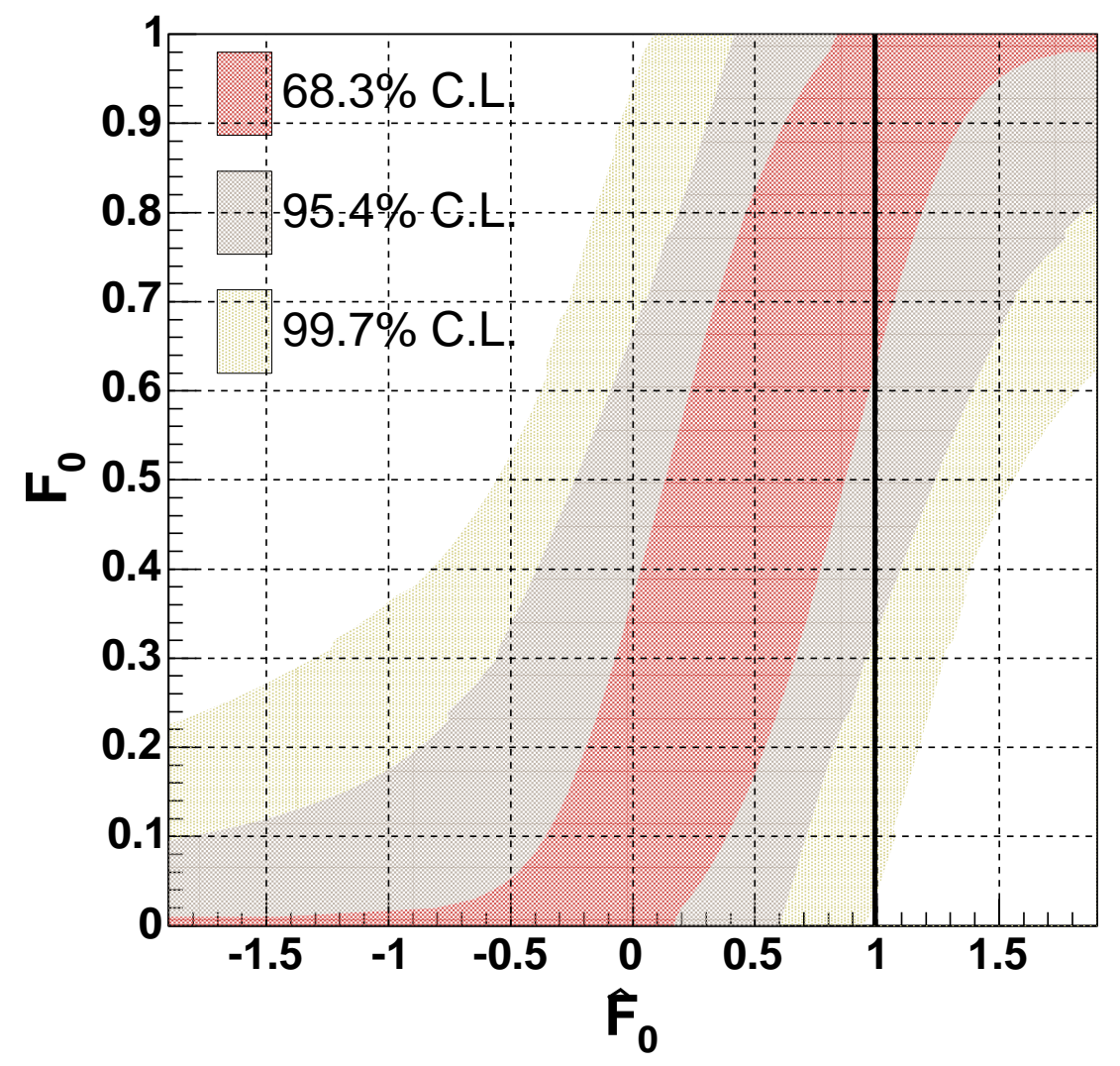

Figure 8.1: $1 \sigma, 2 \sigma$ and $3 \sigma$ Feldman-Cousins confidence intervals for an experiment in the lepton-plus-jets sample. These intervals include systematic uncertainties. The thick vertical line at $\hat{F}_{0}=0.99$ indicates the experimental outcome. 


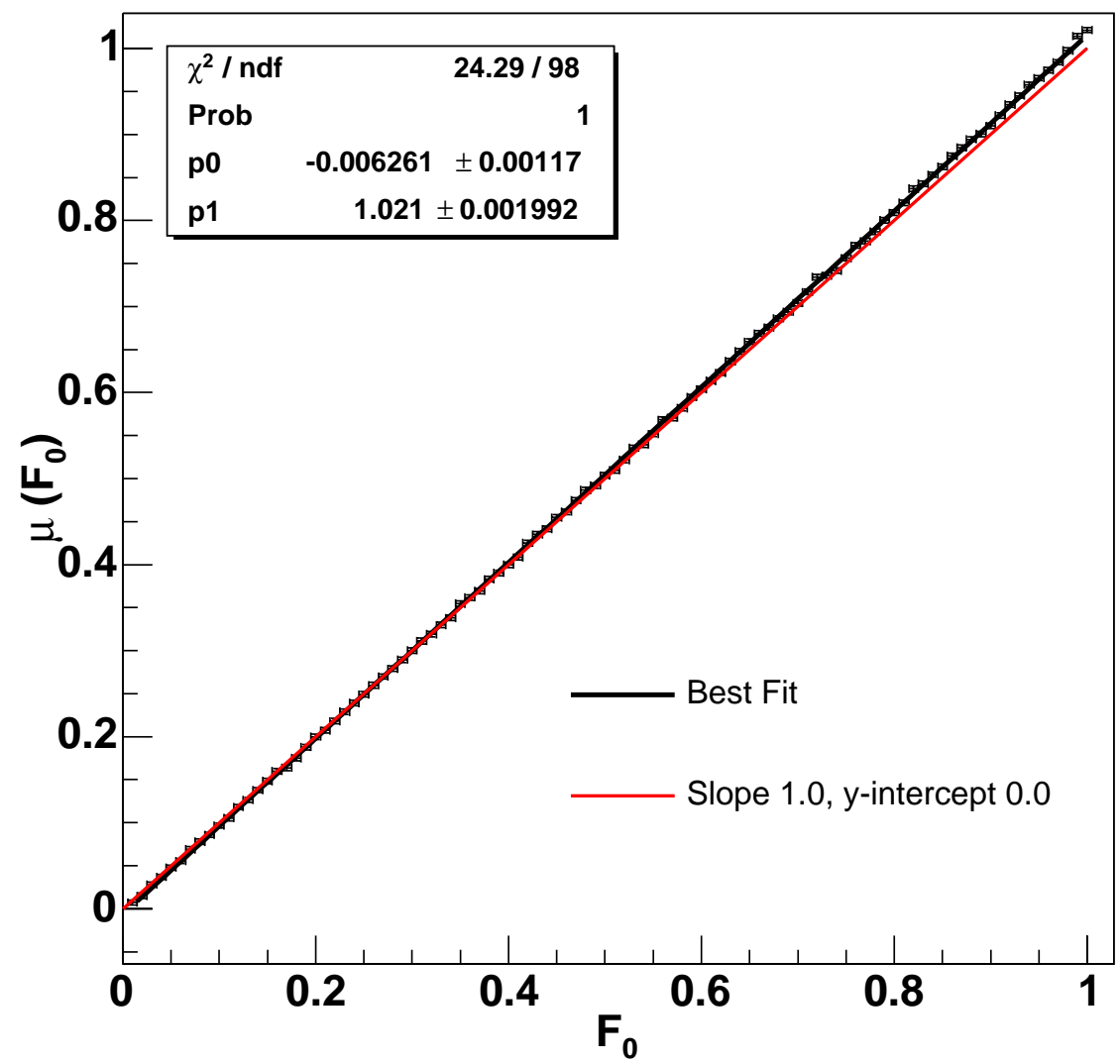

Figure 8.2: Parameterization of $\mu\left(F_{0}\right)$ for pseudo-experiments using the default binned likelihood fitter. The best fit to the data points with a first-order polynomial is drawn black, with $p_{0}$ and $p_{1}$ denoting the polynomial coefficients. For comparison, a line with unit slope and $y$-intercept of zero is shown in red. 


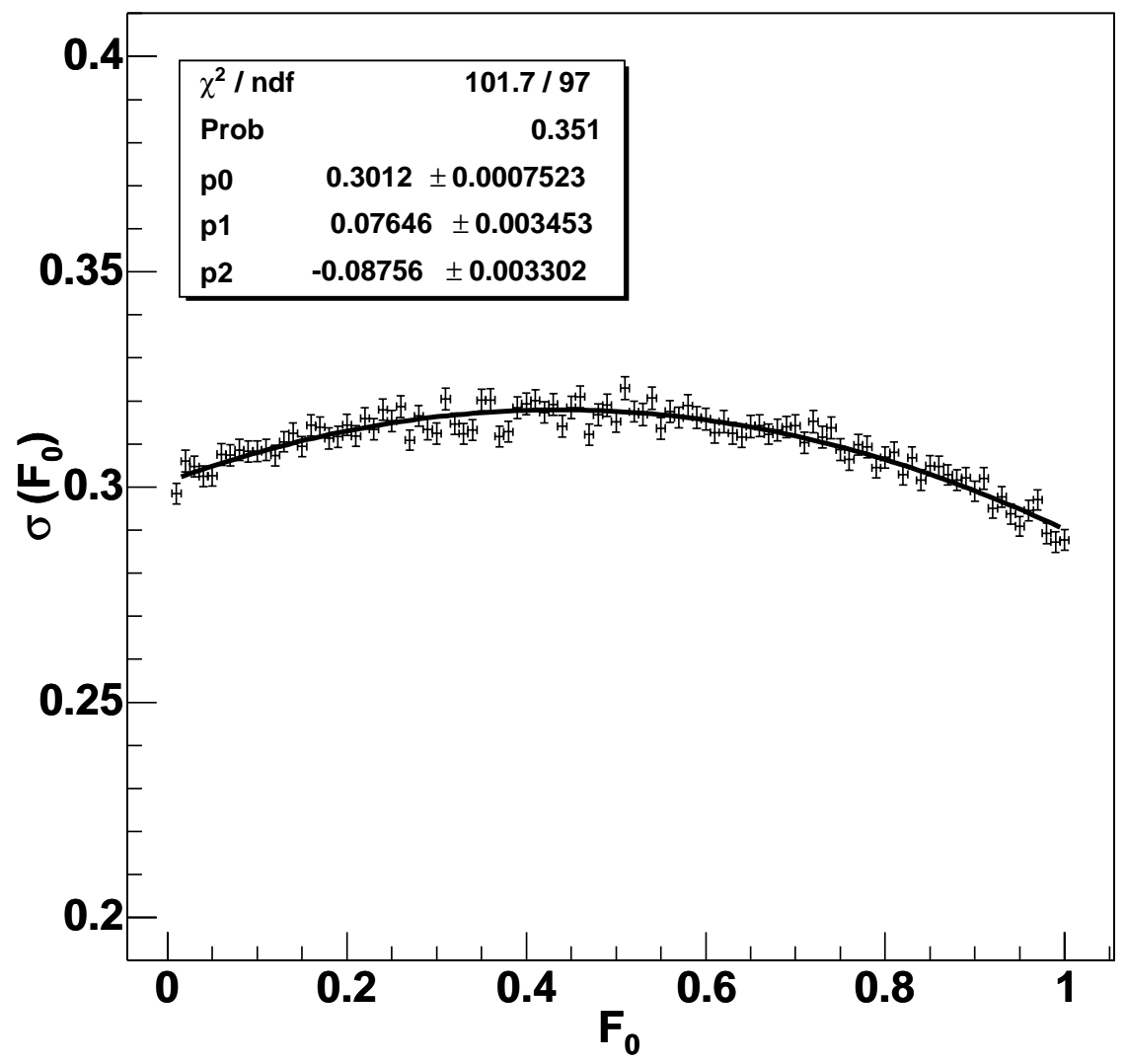

Figure 8.3: Parameterization of $\sigma\left(F_{0}\right)$ for pseudo-experiments using the default binned likelihood fitter. A black line denotes the best fit to the data points; the functional form is that of a second-order polynomial. 


\section{Chapter 9}

\section{Conclusions}

This analysis has measured the fraction of longitudinal $W$ bosons from the decay of top quarks to be $F_{0}=0.99_{-0.35}^{+0.29}$ (stat.) \pm 0.19 (syst.). This result is consistent with the standard model prediction of $F_{0}=0.701 \pm 0.012$ for a top mass of $174.3 \mathrm{GeV} / \mathrm{c}^{2}$. A lower limit for this result is $F_{0}>0.33 @ 95 \%$ CL. The limited statistics of the current lepton-plus-jets data sample, from $\sim 162 \mathrm{pb}^{-1}$ of integrated luminosity, currently prohibits obtaining a more precise measurement. This result has demonstrated that an analysis of lepton angular distributions is a powerful technique for extracting the polarization of $W$ bosons from top-quark candidate decays.

A precise measurement of $W$ boson polarization is important because of the intimate relationship between the longitudinal $W$ and the electroweak symmetry breaking mechanism in the standard model. It has been shown that one-loop supersymmetric QCD and electroweak corrections to the total width of $t \rightarrow W b$ could increase the longitudinal fraction as predicted by the standard model by as much as a few percent [12]. Furthermore, a direct measurement of the weak-current chirality from the $t W b$ vertex is necessary to validate the $V-A$ form predicted by the standard model.

As Run II of the Tevatron continues, CDF will obtain significantly larger amounts of luminosity. From greater statistics alone, the precision of this measurement will increase substantially over the value quoted here. Running multiple sets of pseudoexperiments where the number of $t \bar{t}$ candidate events in the data is extrapolated 


\begin{tabular}{|c|c|c|}
\hline \hline Sample size $\left(\mathrm{fb}^{-1}\right)$ & Num. $t \bar{t}$ candidates & Statistical Unc. \\
\hline 0.162 & 31 & 0.30 \\
0.5 & 100 & 0.17 \\
1.0 & 200 & 0.12 \\
2.0 & 400 & 0.09 \\
\hline \hline
\end{tabular}

Table 9.1: Projections for the statistical uncertainty on the measured longitudinal fraction, using the $\cos \theta^{*}$ technique, for various integrated luminosity values.

from the 31 observed in $162 \mathrm{pb}^{-1}$, projections regarding the statistical uncertainty can be made at $0.5,1.0$ and $2.0 \mathrm{fb}^{-1}$. The results of these pesudo-experiments are summarized in Table 9.1. With $2.0 \mathrm{fb}^{-1}$ of data, approximately $400 t \bar{t}$ candidates will pass the selection criteria and the statistical uncertainty will be less than \pm 0.09 . At such precision the systematic uncertainties will warrant significantly less conservative estimates. Systematics attributed to the background shape, the jet energy scale and the uncertainty on the top mass will all benefit from greater statistics in both data and Monte Carlo samples. It might be possible to further reduce the statistical uncertainty by separating the likelihood fit into the 3.5 and 4-jet bins, or by dividing the lepton-plus-jets sample based on charged-lepton type. Additionally, adjustments to the $\chi_{\min }^{2}$ cut by revisiting optimization studies may yield higher sensitivity.

The likelihood fitter used by this analysis can be expanded to include the right handed fraction, $F_{R}$, as a parameter measured from the fit to the data (Equation 4.12). However, with the limited statistics available in the current data sample it is not possible to fit for $F_{0}$ and $F_{R}$ simultaneously using this technique. With the projections of greater luminosity at the Tevatron, this method can measure the right-handed fraction as a check for a $V+A$ form of the charged-current weak interaction. 


\section{Appendix A}

\section{The CDF Collaboration}

D. Acosta, ${ }^{16}$ J. Adelman, ${ }^{12}$ T. Affolder, ${ }^{9}$ T. Akimoto, ${ }^{54}$ M.G. Albrow,${ }^{15}$ D. Ambrose,${ }^{43} \mathrm{~S}$. Amerio, ${ }^{42}$ D. Amidei,${ }^{33}$ A. Anastassov,${ }^{50} \mathrm{~K}$. Anikeev ${ }^{31}$ A. Annovi,${ }^{44}$ J. Antos, ${ }^{1}$ M. Aoki, ${ }^{54}$ G. Apollinari, ${ }^{15}$ T. Arisawa,${ }^{56}$ J-F. Arguin, ${ }^{32}$ A. Artikov,${ }^{13}$ W. Ashmanskas, ${ }^{15}$ A. Attal,${ }^{7}$ F. Azfar,${ }^{41}$ P. Azzi-Bacchetta,${ }^{42}$ N. Bacchetta,${ }^{42}$ H. Bachacou, ${ }^{28}$ W. Badgett,,${ }^{15}$ A. Barbaro-Galtieri, ${ }^{28}$ G.J. Barker,${ }^{25}$ V.E. Barnes,${ }^{46}$ B.A. Barnett,${ }^{24}$ S. Baroiant,${ }^{6}$ M. Barone,${ }^{17}$ G. Bauer ${ }^{31}$ F. Bedeschi,${ }^{44}$ S. Behari,${ }^{24}$ S. Belforte, ${ }^{53}$ G. Bellettini, ${ }^{44}$ J. Bellinger ${ }^{58}$ E. Ben-Haim, ${ }^{15}$ D. Benjamin, ${ }^{14}$ A. Beretvas, ${ }^{15}$ A. Bhatti, ${ }^{48}$ M. Binkley, ${ }^{15}$ D. Bisello, ${ }^{42}$ M. Bishai, ${ }^{15}$ R.E. Blair, ${ }^{2}$ C. Blocker,${ }^{5}$ K. Bloom,${ }^{33}$ B. Blumenfeld,${ }^{24}$ A. Bocci, ${ }^{48}$ A. Bodek,${ }^{47}$ G. Bolla, ${ }^{46}$ A. Bolshov, ${ }^{31}$ P.S.L. Booth ${ }^{29}$ D. Bortoletto, ${ }^{46}$ J. Boudreau, ${ }^{45}$ S. Bourov,${ }^{15}$ C. Bromberg, ${ }^{34}$ E. Brubaker, ${ }^{12}$ J. Budagov ${ }^{13}$ H.S. Budd, ${ }^{47}$ K. Burkett,${ }^{15}$ G. Busetto ${ }^{42}$ P. Bussey, ${ }^{19}$ K.L. Byrum, ${ }^{2}$ S. Cabrera, ${ }^{14}$ M. Campanelli, ${ }^{18}$ M. Campbell, ${ }^{33}$ A. Canepa,${ }^{46}$ M. Casarsa,${ }^{53}$ D. Carlsmith, ${ }^{58}$ S. Carron, ${ }^{14}$ R. Carosi,,${ }^{44}$ M. Cavalli-Sforza,${ }^{3}$ A. Castro, ${ }^{4}$ P. Catastini,${ }^{44}$ D. Cauz,${ }^{53}$ A. Cerri, ${ }^{28}$ C. Cerri, ${ }^{44}$ L. Cerrito, ${ }^{23}$ J. Chapman, ${ }^{33}$ C. Chen, ${ }^{43}$ Y.C. Chen, ${ }^{1}$ M. Chertok,${ }^{6}$ G. Chiarelli, ${ }^{44}$ G. Chlachidze,${ }^{13}$ F. Chlebana,${ }^{15}$ I. Cho,${ }^{27}$ K. Cho, ${ }^{27}$ D. Chokheli, ${ }^{13}$ M.L. Chu,${ }^{1}$ S. Chuang, ${ }^{58}$ J.Y. Chung, ${ }^{38}$ W-H. Chung, ${ }^{58}$ Y.S. Chung, ${ }^{47}$ C.I. Ciobanu, ${ }^{23}$ M.A. Ciocci, ${ }^{44}$ A.G. Clark, ${ }^{18}$ D. Clark, ${ }^{5}$ M. Coca,${ }^{47}$ A. Connolly,${ }^{28}$ M. Convery,${ }^{48}$ 
J. Conway, ${ }^{6}$ B. Cooper,${ }^{30}$ M. Cordelli, ${ }^{17}$ G. Cortiana, ${ }^{42}$ J. Cranshaw, ${ }^{52}$ J. Cuevas,${ }^{10}$ R. Culbertson, ${ }^{15}$ C. Currat,${ }^{28}$ D. Cyr,${ }^{58}$ D. Dagenhart,${ }^{5}$ S. Da Ronco, ${ }^{42}$ S. D'Auria, ${ }^{19}$ P. de Barbaro ${ }^{47}$ S. De Cecco ${ }^{49}$ G. De Lentdecker ${ }^{47}$ S. Dell'Agnello, ${ }^{17}$ M. Dell'Orso, ${ }^{44}$ S. Demers ${ }^{47}$ L. Demortier, ${ }^{48}$ M. Deninno, ${ }^{4}$ D. De Pedis, ${ }^{49}$ P.F. Derwent,${ }^{15}$ C. Dionisi, ${ }^{49}$ J.R. Dittmann, ${ }^{15}$ P. Doksus, ${ }^{23}$ A. Dominguez, ${ }^{28}$ S. Donati, ${ }^{44}$ M. Donega,${ }^{18}$ J. Donini,${ }^{42}$ M. D'Onofrio, ${ }^{18}$ T. Dorigo, ${ }^{42}$ V. Drollinger, ${ }^{36}$ K. Ebina,${ }^{56}$ N. Eddy ${ }^{23}$ R. Ely, ${ }^{28}$ R. Erbacher,${ }^{6}$ M. Erdmann,${ }^{25}$ D. Errede,${ }^{23}$ S. Errede,${ }^{23}$ R. Eusebi ${ }^{47}$ H-C. Fang, ${ }^{28}$ S. Farrington, ${ }^{29}$ I. Fedorko, ${ }^{44}$ R.G. Feild,${ }^{59}$ M. Feindt, ${ }^{25}$ J.P. Fernandez ${ }^{46}$ C. Ferretti, ${ }^{33}$ R.D. Field,${ }^{16}$ I. Fiori,${ }^{44}$ G. Flanagan,${ }^{34}$ B. Flaugher, ${ }^{15}$ L.R. Flores-Castillo, ${ }^{45}$ A. Foland,${ }^{20}$ S. Forrester,${ }^{6}$ G.W. Foster, ${ }^{15}$ M. Franklin, ${ }^{20}$ J.C. Freeman, ${ }^{28}$ H. Frisch, ${ }^{12}$ Y. Fujii ${ }^{26}$ I. Furic ${ }^{12}$ A. Gajjar ${ }^{29}$ A. Gallas,${ }^{37}$ J. Galyardt, ${ }^{11}$ M. Gallinaro, ${ }^{48}$ M. Garcia-Sciveres,${ }^{28}$ A.F. Garfinkel, ${ }^{46}$ C. Gay, ${ }^{59}$ H. Gerberich, ${ }^{14}$ D.W. Gerdes, ${ }^{33}$ E. Gerchtein, ${ }^{11}$ S. Giagu ${ }^{49}$ P. Giannetti, ${ }^{44}$ A. Gibson, ${ }^{28}$ K. Gibson, ${ }^{11}$ C. Ginsburg,${ }^{58}$ K. Giolo,${ }^{46}$ M. Giordani, ${ }^{53}$ G. Giurgiu,${ }^{11}$ V. Glagolev, ${ }^{13}$ D. Glenzinski, ${ }^{15}$ M. Gold, ${ }^{36}$ N. Goldschmidt, ${ }^{33}$ D. Goldstein, ${ }^{7}$ J. Goldstein ${ }^{41}$ G. Gomez,${ }^{10}$ G. Gomez-Ceballos,${ }^{31}$ M. Goncharov, ${ }^{51}$ O. González, ${ }^{46}$ I. Gorelov, ${ }^{36}$ A.T. Goshaw,${ }^{14}$ Y. Gotra,${ }^{45}$ K. Goulianos,${ }^{48}$ A. Gresele,${ }^{4}$ M. Griffiths, ${ }^{29}$ C. Grosso-Pilcher ${ }^{12}$ U. Grundler ${ }^{23}$ M. Guenther, ${ }^{46}$ J. Guimaraes da Costa,${ }^{20}$ C. Haber, ${ }^{28}$ K. Hahn, ${ }^{43}$ S.R. Hahn,${ }^{15}$ E. Halkiadakis, ${ }^{47}$ A. Hamilton, ${ }^{32}$ B-Y. Han,${ }^{47}$ R. Handler, ${ }^{58}$ F. Happacher, ${ }^{17}$ K. Hara,${ }^{54}$ M. Hare,${ }^{55}$ R.F. Harr,${ }^{57}$ R.M. Harris,${ }^{15}$ F. Hartmann, ${ }^{25}$ K. Hatakeyama, ${ }^{48}$ J. Hauser, ${ }^{7}$ C. Hays, ${ }^{14}$ H. Hayward ${ }^{29}$ E. Heider, ${ }^{55}$ B. Heinemann, ${ }^{29}$ J. Heinrich, ${ }^{43}$ M. Hennecke, ${ }^{25}$ M. Herndon, ${ }^{24}$ C. Hill, ${ }^{9}$ D. Hirschbuehl,${ }^{25}$ A. Hocker ${ }^{47}$ K.D. Hoffman, ${ }^{12}$ A. Holloway,${ }^{20}$ S. Hou, ${ }^{1}$ M.A. Houlden,${ }^{29}$ B.T. Huffman, ${ }^{41}$ Y. Huang, ${ }^{14}$ R.E. Hughes,${ }^{38}$ J. Huston, ${ }^{34}$ K. Ikado, ${ }^{56}$ J. Incandela,${ }^{9}$ G. Introzzi ${ }^{44}$ M. Iori, ${ }^{49}$ Y. Ishizawa,${ }^{54}$ C. Issever,${ }^{9}$ A. Ivanov ${ }^{47}$ Y. Iwata,${ }^{22}$ B. Iyutin, ${ }^{31}$ E. James, ${ }^{15}$ D. Jang, ${ }^{50}$ J. Jarrell,${ }^{36}$ D. Jeans,${ }^{49}$ H. Jensen, ${ }^{15}$ E.J. Jeon, ${ }^{27}$ M. Jones,${ }^{46}$ K.K. Joo,${ }^{27}$ S. Jun, ${ }^{11}$ T. Junk, ${ }^{23}$ T. Kamon,${ }^{51}$ 
J. Kang, ${ }^{33}$ M. Karagoz Unel, ${ }^{37}$ P.E. Karchin,,${ }^{57}$ S. Kartal, ${ }^{15}$ Y. Kato, ${ }^{40}$ Y. Kemp,${ }^{25}$ R. Kephart,${ }^{15}$ U. Kerzel,,${ }^{25}$ V. Khotilovich, ${ }^{51}$ B. Kilminster, ${ }^{38}$ D.H. Kim, ${ }^{27}$ H.S. Kim, ${ }^{23}$ J.E. Kim, ${ }^{27}$ M.J. Kim, ${ }^{11}$ M.S. Kim, ${ }^{27}$ S.B. Kim, ${ }^{27}$ S.H. Kim,${ }^{54}$ T.H. Kim, ${ }^{31}$ Y.K. Kim, ${ }^{12}$ B.T. King, ${ }^{29}$ M. Kirby, ${ }^{14}$ L. Kirsch, ${ }^{5}$ S. Klimenko, ${ }^{16}$ B. Knuteson, ${ }^{31}$ B.R. Ko, ${ }^{14}$ H. Kobayashi, ${ }^{54}$ P. Koehn, ${ }^{38}$ D.J. Kong, ${ }^{27}$ K. Kondo, ${ }^{56}$ J. Konigsberg, ${ }^{16}$ K. Kordas, ${ }^{32}$ A. Korn, ${ }^{31}$ A. Korytov, ${ }^{16}$ K. Kotelnikov, ${ }^{35}$ A.V. Kotwal, ${ }^{14}$ A. Kovalev, ${ }^{43}$ J. Kraus ${ }^{23}$ I. Kravchenko, ${ }^{31}$ A. Kreymer, ${ }^{15}$ J. Kroll, ${ }^{43}$ M. Kruse, ${ }^{14}$ V. Krutelyov,${ }^{51}$ S.E. Kuhlmann, ${ }^{2}$ N. Kuznetsova, ${ }^{15}$ A.T. Laasanen, ${ }^{46}$ S. Lai, ${ }^{32}$ S. Lami ${ }^{48}$ S. Lammel,,${ }^{15}$ J. Lancaster, ${ }^{14}$ M. Lancaster, ${ }^{30}$ R. Lander ${ }^{6}$ K. Lannon, ${ }^{38}$ A. Lath, ${ }^{50}$ G. Latino, ${ }^{36}$ R. Lauhakangas, ${ }^{21}$ I. Lazzizzera, ${ }^{42}$ Y. Le,${ }^{24}$ C. Lecci, ${ }^{25}$ T. LeCompte,${ }^{2}$ J. Lee,${ }^{27}$ J. Lee, ${ }^{47}$ S.W. Lee ${ }^{51}$ R. Lefevre, ${ }^{3}$ N. Leonardo, ${ }^{31}$ S. Leone, ${ }^{44}$ J.D. Lewis,$^{15}$ K. Li ${ }^{59}$ C. Lin ${ }^{59}$ C.S. Lin,${ }^{15}$ M. Lindgren,${ }^{15}$ T.M. Liss, ${ }^{23}$ D.O. Litvintsev,${ }^{15}$ T. Liu, ${ }^{15}$ Y. Liu, ${ }^{18}$ N.S. Lockyer, ${ }^{43}$ A. Loginov, ${ }^{35}$ M. Loreti,${ }^{42}$ P. Loverre ${ }^{49}$ RS. Lu, ${ }^{1}$ D. Lucchesi,${ }^{42}$ P. Lujan, ${ }^{28}$ P. Lukens, ${ }^{15}$ G. Lungu, ${ }^{16}$ L. Lyons,${ }^{41}$ J. Lys, ${ }^{28}$

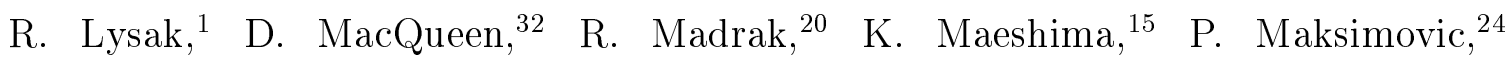
L. Malferrari, ${ }^{4}$ G. Manca, ${ }^{29}$ R. Marginean, ${ }^{38}$ M. Martin,,${ }^{24}$ A. Martin,,${ }^{59}$ V. Martin,,${ }^{37}$ M. Martínez, ${ }^{3}$ T. Maruyama,${ }^{54}$ H. Matsunaga,${ }^{54}$ M. Mattson,${ }^{57}$ P. Mazzanti ${ }^{4}$ K.S. McFarland ${ }^{47}$ D. McGivern, ${ }^{30}$ P.M. McIntyre,${ }^{51}$ P. McNamara,${ }^{50}$ R. NcNulty, ${ }^{29}$ S. Menzemer, ${ }^{31}$ A. Menzione,${ }^{44}$ P. Merkel,${ }^{15}$ C. Mesropian, ${ }^{48}$ A. Messina,${ }^{49}$ T. Miao,${ }^{15}$ N. Miladinovic,${ }^{5}$ L. Miller,${ }^{20}$ R. Miller ${ }^{34}$ J.S. Miller, ${ }^{33}$ R. Miquel, ${ }^{28}$ S. Miscetti, ${ }^{17}$ G. Mitselmakher, ${ }^{16}$ A. Miyamoto,${ }^{26}$ Y. Miyazaki,${ }^{40}$ N. Moggi,${ }^{4}$ B. Mohr, ${ }^{7}$ R. Moore,${ }^{15}$ M. Morello, ${ }^{44}$ A. Mukherjee, ${ }^{15}$ M. Mulhearn, ${ }^{31}$ T. Muller, ${ }^{25}$ R. Mumford,${ }^{24}$ A. Munar, ${ }^{43}$ P. Murat,${ }^{15}$ J. Nachtman,${ }^{15}$ S. Nahn,${ }^{59}$ I. Nakamura, ${ }^{43}$ I. Nakano,,${ }^{39}$ A. Napier,${ }^{55}$ R. Napora,${ }^{24}$ D. Naumov ${ }^{36}$ V. Necula,${ }^{16}$ F. Niell, ${ }^{33}$ J. Nielsen, ${ }^{28}$ C. Nelson, ${ }^{15}$ T. Nelson,,${ }^{15}$ C. Neu, ${ }^{43}$ M.S. Neubauer, ${ }^{8}$ C. Newman-Holmes,${ }^{15}$ AS. Nicollerat, ${ }^{18}$ T. Nigmanov, ${ }^{45}$ L. Nodulman, ${ }^{2}$ O. Norniella, ${ }^{3}$ K. Oesterberg, ${ }^{21}$ T. Ogawa,${ }^{56}$ S.H. Oh, ${ }^{14}$ Y.D. Oh,${ }^{27}$ T. Ohsugi, ${ }^{22}$ T. Okusawa,${ }^{40}$ R. Oldeman,${ }^{49}$ 
R. Orava,${ }^{21}$ W. Orejudos ${ }^{28}$ C. Pagliarone, ${ }^{44}$ F. Palmonari, ${ }^{44}$ R. Paoletti, ${ }^{44}$ V. Papadimitriou, ${ }^{15}$ S. Pashapour ${ }^{32}$ J. Patrick, ${ }^{15}$ G. Pauletta, ${ }^{53}$ M. Paulini, ${ }^{11}$ T. Pauly, ${ }^{41}$ C. Paus, ${ }^{31}$ D. Pellett,${ }^{6}$ A. Penzo,${ }^{53}$ T.J. Phillips, ${ }^{14}$ G. Piacentino, ${ }^{44}$ J. Piedra, ${ }^{10}$ K.T. Pitts, ${ }^{23}$ C. Plager,${ }^{7}$ A. Pompoš,${ }^{46}$ L. Pondrom,${ }^{58}$ G. Pope,${ }^{45}$ O. Poukhov ${ }^{13}$ F. Prakoshyn, ${ }^{13}$ T. Pratt,${ }^{29}$ A. Pronko, ${ }^{16}$ J. Proudfoot,${ }^{2}$ F. Ptohos,${ }^{17}$ G. Punzi, ${ }^{44}$ J. Rademacker, ${ }^{41}$ A. Rakitine, ${ }^{31}$ S. Rappoccio,${ }^{20}$ F. Ratnikov ${ }^{50}$ H. Ray, ${ }^{33}$ A. Reichold, ${ }^{41}$ B. Reisert,${ }^{15}$ V. Rekovic,${ }^{36}$ P. Renton, ${ }^{41}$ M. Rescigno, ${ }^{49}$ F. Rimondi, ${ }^{4}$ K. Rinnert,${ }^{25}$ L. Ristori, ${ }^{44}$ W.J. Robertson,,${ }^{14}$ A. Robson, ${ }^{41}$ T. Rodrigo,${ }^{10}$ S. Rolli, ${ }^{55}$ L. Rosenson, ${ }^{31}$ R. Roser,${ }^{15}$ R. Rossin, ${ }^{42}$ C. Rott, ${ }^{46}$ J. Russ, ${ }^{11}$ A. Ruiz, ${ }^{10}$ D. Ryan, ${ }^{55}$ H. Saarikko, ${ }^{21}$ S. Sabik, ${ }^{32}$ A. Safonov,${ }^{6}$ R. St. Denis,${ }^{19}$ W.K. Sakumoto, ${ }^{47}$ G. Salamanna, ${ }^{49}$ D. Saltzberg, ${ }^{7}$ C. Sanchez, ${ }^{3}$ A. Sansoni, ${ }^{17}$ L. Santi, ${ }^{53}$ S. Sarkar, ${ }^{49}$ K. Sato, ${ }^{54}$ P. Savard, ${ }^{32}$ A. Savoy-Navarro, ${ }^{15}$ P. Schlabach, ${ }^{15}$ E.E. Schmidt, ${ }^{15}$ M.P. Schmidt ${ }^{59}$ M. Schmitt,${ }^{37}$ L. Scodellaro, ${ }^{42}$ A. Scribano, ${ }^{44}$ F. Scuri, ${ }^{44}$ A. Sedov, ${ }^{46}$ S. Seidel, ${ }^{36}$ Y. Seiya, ${ }^{40}$ F. Semeria, ${ }^{4}$ L. Sexton-Kennedy, ${ }^{15}$ I. Sfiligoi, ${ }^{17}$ M.D. Shapiro, ${ }^{28}$ T. Shears, ${ }^{29}$ P.F. Shepard,${ }^{45}$ M. Shimojima, ${ }^{54}$ M. Shochet, ${ }^{12}$ Y. Shon,${ }^{58}$ I. Shreyber ${ }^{35}$ A. Sidoti, ${ }^{44}$ J. Siegrist, ${ }^{28}$ M. Siket, ${ }^{1}$ A. Sill, ${ }^{52}$ P. Sinervo, ${ }^{32}$ A. Sisakyan, ${ }^{13}$ A. Skiba, ${ }^{25}$ A.J. Slaughter, ${ }^{15}$ K. Sliwa, ${ }^{55}$ D. Smirnov, ${ }^{36}$ J.R. Smith, ${ }^{6}$ F.D. Snider, ${ }^{15}$ R. Snihur, ${ }^{32}$ S.V. Somalwar, ${ }^{50}$ J. Spalding, ${ }^{15}$ M. Spezziga,${ }^{52}$ L. Spiegel,${ }^{15}$ F. Spinella,${ }^{44}$ M. Spiropulu, ${ }^{9}$ P. Squillacioti, ${ }^{44}$ H. Stadie,${ }^{25}$ A. Stefanini, ${ }^{44}$ B. Stelzer,${ }^{32}$ O. StelzerChilton, ${ }^{32}$ J. Strologas, ${ }^{36}$ D. Stuart, ${ }^{9}$ A. Sukhanov, ${ }^{16}$ K. Sumorok, ${ }^{31}$ H. Sun,${ }^{55}$ T. Suzuki, ${ }^{54}$ A. Taffard, ${ }^{23}$ R. Tafirout,${ }^{32}$ S.F. Takach,${ }^{57}$ H. Takano, ${ }^{54}$ R. Takashima, ${ }^{22}$ Y. Takeuchi, ${ }^{54}$ K. Takikawa, ${ }^{54}$ M. Tanaka,$^{2}$ R. Tanaka, ${ }^{39}$ N. Tanimoto, ${ }^{39}$ S. Tapprogge, ${ }^{21}$ M. Tecchio, ${ }^{33}$ P.K. Teng, ${ }^{1}$ K. Terashi, ${ }^{48}$ R.J. Tesarek, ${ }^{15}$ S. Tether,${ }^{31}$ J. Thom, ${ }^{15}$ A.S. Thompson, ${ }^{19}$ E. Thomson, ${ }^{43}$ P. Tipton, ${ }^{47}$ V. Tiwari, ${ }^{11}$ S. Tkaczyk,${ }^{15}$ D. Toback,${ }^{51}$ K. Tollefson, ${ }^{34}$ T. Tomura,${ }^{54}$ D. Tonelli, ${ }^{44}$ M. Tönnesmann, ${ }^{34}$ S. Torre,${ }^{44}$ D. Torretta, ${ }^{15} \mathrm{~S}$. Tourneur, ${ }^{15} \mathrm{~W}$. Trischuk, ${ }^{32} \mathrm{~J}$. Tseng, ${ }^{41} \mathrm{R}$. Tsuchiya, ${ }^{56}$ S. Tsuno, ${ }^{39}$ D. Tsybychev, ${ }^{16}$ N. Turini, ${ }^{44}$ M. Turner, ${ }^{29}$ F. Ukegawa, ${ }^{54}$ T. Unverhau, ${ }^{19}$ S. Uozumi,${ }^{54}$ 
D. Usynin,${ }^{43}$ L. Vacavant,${ }^{28}$ A. Vaiciulis, ${ }^{47}$ A. Varganov, ${ }^{33}$ E. Vataga, ${ }^{44}$ S. Vejcik III,${ }^{15}$ G. Velev, ${ }^{15}$ V. Veszpremi, ${ }^{46}$ G. Veramendi, ${ }^{23}$ T. Vickey, ${ }^{23}$ R. Vidal,,${ }^{15}$ I. Vila, ${ }^{10}$ R. Vilar, ${ }^{10}$ I. Vollrath, ${ }^{32}$ I. Volobouev, ${ }^{28}$ M. von der Mey, ${ }^{7}$ P. Wagner, ${ }^{51}$ R.G. Wagner, ${ }^{2}$ R.L. Wagner,${ }^{15}$ W. Wagner,${ }^{25}$ R. Wallny,${ }^{7}$ T. Walter,${ }^{25}$ T. Yamashita, ${ }^{39}$ K. Yamamoto, ${ }^{40}$ Z. Wan, ${ }^{50}$ M.J. Wang, ${ }^{1}$ S.M. Wang, ${ }^{16}$ A. Warburton, ${ }^{32}$ B. Ward, ${ }^{19}$ S. Waschke, ${ }^{19}$ D. Waters, ${ }^{30}$ T. Watts, ${ }^{50}$ M. Weber, ${ }^{28}$ W.C. Wester III, ${ }^{15}$ B. Whitehouse ${ }^{55}$ A.B. Wicklund, ${ }^{2}$ E. Wicklund, ${ }^{15}$ H.H. Williams, ${ }^{43}$ P. Wilson, ${ }^{15}$ B.L. Winer, ${ }^{38}$ P. Wittich, ${ }^{43}$ S. Wolbers,${ }^{15}$ M. Wolter,${ }^{55}$ M. Worcester, ${ }^{7}$ S. Worm,${ }^{50}$ T. Wright ${ }^{33}$ X. Wu, ${ }^{18}$ F. Würthwein, ${ }^{8}$ A. Wyatt,${ }^{30}$ A. Yagil, ${ }^{15}$ U.K. Yang, ${ }^{12}$ W. Yao, ${ }^{28}$ G.P. Yeh, ${ }^{15}$ K. Yi,${ }^{24}$ J. Yoh, ${ }^{15}$ P. Yoon,${ }^{47}$ K. Yorita,${ }^{56}$ T. Yoshida, ${ }^{40}$ I. Yu, ${ }^{27}$ S. Yu, ${ }^{43}$ Z. Yu, ${ }^{59}$ J.C. Yun, ${ }^{15}$ L. Zanello, ${ }^{49}$ A. Zanetti, ${ }^{53}$ I. Zaw, ${ }^{20}$ F. Zetti, ${ }^{44}$ J. Zhou, ${ }^{50}$ A. Zsenei, ${ }^{18}$ and S. Zucchelli, ${ }^{4}$

\section{(CDF Collaboration)}

1 Institute of Physics, Academia Sinica, Taipei, Taiwan 11529, Republic of China

2 Argonne National Laboratory, Argonne, Illinois 60439

3 Institut de Fisica d'Altes Energies, Universitat Autonoma de Barcelona, E-08193, Bellaterra (Barcelona), Spain

4 Istituto Nazionale di Fisica Nucleare, University of Bologna, I-40127 Bologna, Italy

5 Brandeis University, Waltham, Massachusetts 02254

6 University of California at Davis, Davis, California 95616

7 University of California at Los Angeles, Los Angeles, California 90024

8 University of California at San Diego, La Jolla, California 92093

9 University of California at Santa Barbara, Santa Barbara, California 93106

10 Instituto de Fisica de Cantabria, CSIC-University of Cantabria, 39005 Santander, Spain

11 Carnegie Mellon University, Pittsburgh, PA 15213

12 Enrico Fermi Institute, University of Chicago, Chicago, Illinois 60637

13 Joint Institute for Nuclear Research, RU-141980 Dubna, Russia 
14 Duke University, Durham, North Carolina 27708

15 Fermi National Accelerator Laboratory, Batavia, Illinois 60510

16 University of Florida, Gainesville, Florida 32611

17 Laboratori Nazionali di Frascati, Istituto Nazionale di Fisica Nucleare, I-00044 Frascati, Italy

18 University of Geneva, CH-1211 Geneva 4, Switzerland

19 Glasgow University, Glasgow G12 8QQ, United Kingdom

20 Harvard University, Cambridge, Massachusetts 02138

21 The Helsinki Group: Helsinki Institute of Physics; and Division of High Energy Physics, Department of

Physical Sciences, University of Helsinki, FIN-00044, Helsinki, Finland

22 Hiroshima University, Higashi-Hiroshima 724, Japan

23 University of Illinois, Urbana, Illinois 61801

24 The Johns Hopkins University, Baltimore, Maryland 21218

25 Institut für Experimentelle Kernphysik, Universität Karlsruhe, 76128 Karlsruhe, Germany

26 High Energy Accelerator Research Organization (KEK), Tsukuba, Ibaraki 305, Japan

27 Center for High Energy Physics: Kyungpook National University, Taegu 702-701; Seoul National University,

Seoul 151-742; and SungKyunKwan University, Suwon 440-746; Korea

28

Ernest Orlando Lawrence Berkeley National Laboratory, Berkeley, California 94720

29 University of Liverpool, Liverpool L69 7ZE, United Kingdom

30 University College London, London WC1E 6BT, United Kingdom

31 Massachusetts Institute of Technology, Cambridge, Massachusetts 02139

32 Institute of Particle Physics: McGill University, Montréal, Canada H3A 2T8; and University of Toronto,

Toronto, Canada M5S 1 A7

33 University of Michigan, Ann Arbor, Michigan 48109

34 Michigan State University, East Lansing, Michigan 48824

35

Institution for Theoretical and Experimental Physics, ITEP, Moscow 117259, Russia

36 University of New Mexico, Albuquerque, New Mexico 87131

37 Northwestern University, Evanston, Illinois 60208 
The Ohio State University, Columbus, Ohio 43210

39

Okayama University, Okayama 700-8530, Japan

40

Osaka City University, Osaka 588, Japan

41 University of Oxford, Oxford OX1 3RH, United Kingdom

42 University of Padova, Istituto Nazionale di Fisica Nucleare, Sezione di Padova-Trento, I-35131 Padova, Italy

43 University of Pennsylvania, Philadelphia, Pennsylvania 19104

44 Istituto Nazionale di Fisica Nucleare, University and Scuola Normale Superiore of Pisa, I-56100 Pisa, Italy

45 University of Pittsburgh, Pittsburgh, Pennsylvania 15260

46 Purdue University, West Lafayette, Indiana 47907

47 University of Rochester, Rochester, New York 14627

48 The Rockefeller University, New York, New York 10021

49 Istituto Nazionale di Fisica Nucleare, Sezione di Roma 1, University di Roma "La Sapienza," I-00185 Roma,

Italy

50 Rutgers University, Piscataway, New Jersey 08855

51 Texas A $6 M$ University, College Station, Texas 77843

52 Texas Tech University, Lubbock, Texas 79409

53 Istituto Nazionale di Fisica Nucleare, University of Trieste/ Udine, Italy

54 University of Tsukuba, Tsukuba, Ibaraki 305, Japan

55 Tufts University, Medford, Massachusetts 02155

56 Waseda University, Tokyo 169, Japan

57 Wayne State University, Detroit, Michigan 48201

58 University of Wisconsin, Madison, Wisconsin 53706

59 Yale University, New Haven, Connecticut 06520 


\section{Appendix B}

\section{COT Wireplane Testing}

As discussed in Section 2.2.1, the Central Outer Tracker (COT) is a major component of the CDF tracking system [41] and is used for charged particle tracking in the region $|\eta| \leq 1.0$. The COT is an open-cell drift chamber containing more than 30,000 gold-plated tungsten wires which are 1.6 mil in diameter ${ }^{1}$ and divided into wireplanes in eight "superlayers." Each wireplane consists of 29 individual wires; 3 field-shaping wires are electrically connected together on each end, and then 23 wires which alternate sense and field-shaping inbetween with printed circuit cards on both ends holding the wires in place and allowing for the connection of electronics. The wireplanes in the COT detector were produced at Fermilab in Batavia, Illinois and were shipped down to Urbana, Illinois for testing. This Appendix briefly describes tension, dark current and source tests on the COT wireplanes which were conducted at the University of Illinois between September 1997 and July 1998 [86].

\section{B.1 Tension Measurements}

The wireplanes which make up the eight superlayers of the COT are held in position within the CDF tacking volume by being suspended between two aluminum endplates.

\footnotetext{
${ }^{1} \mathrm{~A}$ "mil" is a unit of length equal to $10^{-3}$ of an inch $(0.0254 \mathrm{~mm})$.
} 


\begin{tabular}{|c|c|}
\hline \hline Superlayer \# & Length (in) \\
\hline 1 & 122.307 \\
2 & 122.004 \\
3 & 121.940 \\
4 & 121.824 \\
5 & 121.898 \\
6 & 121.904 \\
7 & 122.128 \\
8 & 122.270 \\
\hline \hline
\end{tabular}

Table B.1: COT wireplane lengths, in inches, for each superlayer [86].

Due to the deflection of these endplates under a design tension of $150 \mathrm{gm}$ per wire, ${ }^{2}$ each superlayer has a different length (see Table B.1). The wire tensions for each superlayer are measured at the design length by electrically exciting the wire to vibrate while a portion of the wire is immersed in a magnetic field. The tension of the wire, $T$, is related to the lowest mechanical resonant frequency, $f_{0}$, by

$$
T=\frac{4 L^{2} f_{0}^{2} \mu}{g}(\text { in grams })
$$

where $L$ is the wire length, $\mu$ is the mass per unit length of the wire and $g$ is the acceleration due to gravity.

Tension measurements are carried out on each wire by first mounting the wireplane on a tray which is nominally 131.7 inches in length. The wireplane is clamped at both ends into a precision mounting fixture which includes a micrometer at one end for adjusting the total length, and therefore allowing the wireplane to be fixed to its design length. The length scale was set at a nominal temperature of $72^{\circ} \mathrm{F}$, and is known to approximately \pm 0.005 inches.

We exploit Faraday's Law to determine the resonant frequency of each wire by placing a portion of the wire in a magnetic field, supplied by nine small rare earth

\footnotetext{
${ }^{2}$ Note that the COT wire tensions are given in grams instead of a more conventional unit like Newtons.
} 
magnets mounted just above the wireplane, and driving the wire with an alternating current (see the setup schematic in Figure B.1). The out-of-phase component of the voltage (with respect to the driving voltage) is monitored across a $50 \Omega$ terminator by a lock-in amplifier. The out-of-phase voltage can be written as a function of the driving frequency $\omega$, and is proportional to [87]

$$
\frac{\left(\omega-\omega_{0}^{2} / \omega\right)}{\left(\omega-\omega_{0}^{2} / \omega\right)^{2} \tau^{2}+1}
$$

where $\omega_{0}$ is the resonant frequency and $\tau$ is the damping time for the wire. As the driving frequency is swept through the resonant frequency, the out-of-phase component of the voltage (Eqn. B.2) changes sign, vanishing at the resonant frequency. The procedure for determining the resonant frequency of each wire is as follows: A 25-pin connector attached to the printed circuit card on one end of the wireplane mounted in the tension tray, feeds a sinusoidal voltage signal to all 29 wires in parallel. This sinusoidal voltage is also fed into the lock-in amplifier as a reference signal. A separate 25-pin connector attached at the opposite end of the wireplane feeds the signals to a multiplexer which is set such that it closes the circuit for one of the 25 connections by feeding it to the terminated input of the lock-in amplifier. The driving voltage is swept through a frequency range of about $5 \mathrm{~Hz}$ in steps of $0.125 \mathrm{~Hz}$. Despite an overall offset in the relative phases of the measured and reference signals due to cable lengths, the resonance is quite clear (see Figure B.2). Tension measurements take about 20 minutes per plane.

A measured mass per unit length, $\mu$, is supplied by the wire manufacturer for each spool of wire used by Fermilab to string the COT wireplanes; we use these same values to calculate the tension. From spool-to-spool, the mass per unit length varies from $2.45 \times 10^{-4}$ to $2.60 \times 10^{-4} \mathrm{gm} / \mathrm{cm}$. Each wireplane has a floating wire support mounted at its center, consisting of a PVC rod extending transversely across the 


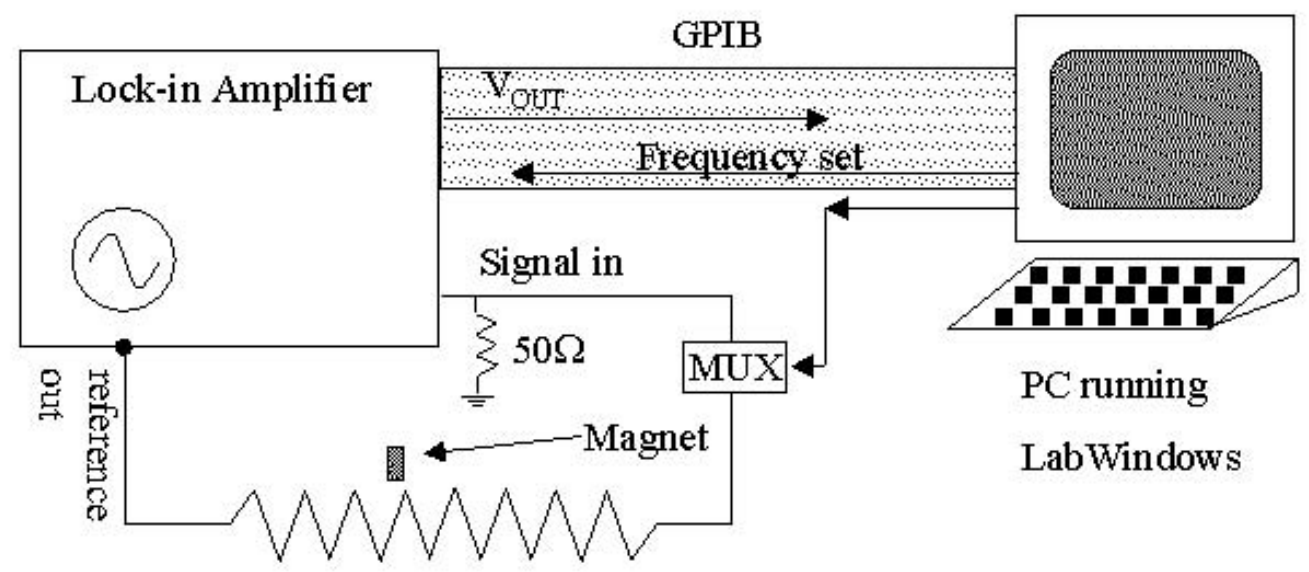

Figure B.1: Wireplane tension testing setup [88].

plane and connected with epoxy to each of the 29 wires. During measurements of the mechanical resonant frequency, the support acts as a vibrational node. The effective length of the wire used in Equation B.1 is thus half of the total wire length.

Tension measurements are performed on each of the individual sense and potential wires. The only exception to this is that the three-wire sets of field-shaping wires at the outer edge of each wireplane have their tension measured together since they are connected electrically. Additionally, the wire tension measurements are performed on both sides of the center wire support. In total, 25 individual measurements are made on one side of the wire support, and one measurement for every fifth wire on the opposite side of the support. Assuming equal tensions on either side of the support, the position and orientation of the wire support can be calculated from these data. No significant deviations from the nominal position and orientation of the wire support were found. The distribution of average tension for each superlayer is shown in Figure B.3.

During the tension testing, a total of 27 wireplanes were rejected out of the roughly one-thousand tested. Of these, 16 wireplanes were rejected due to electrical contact 


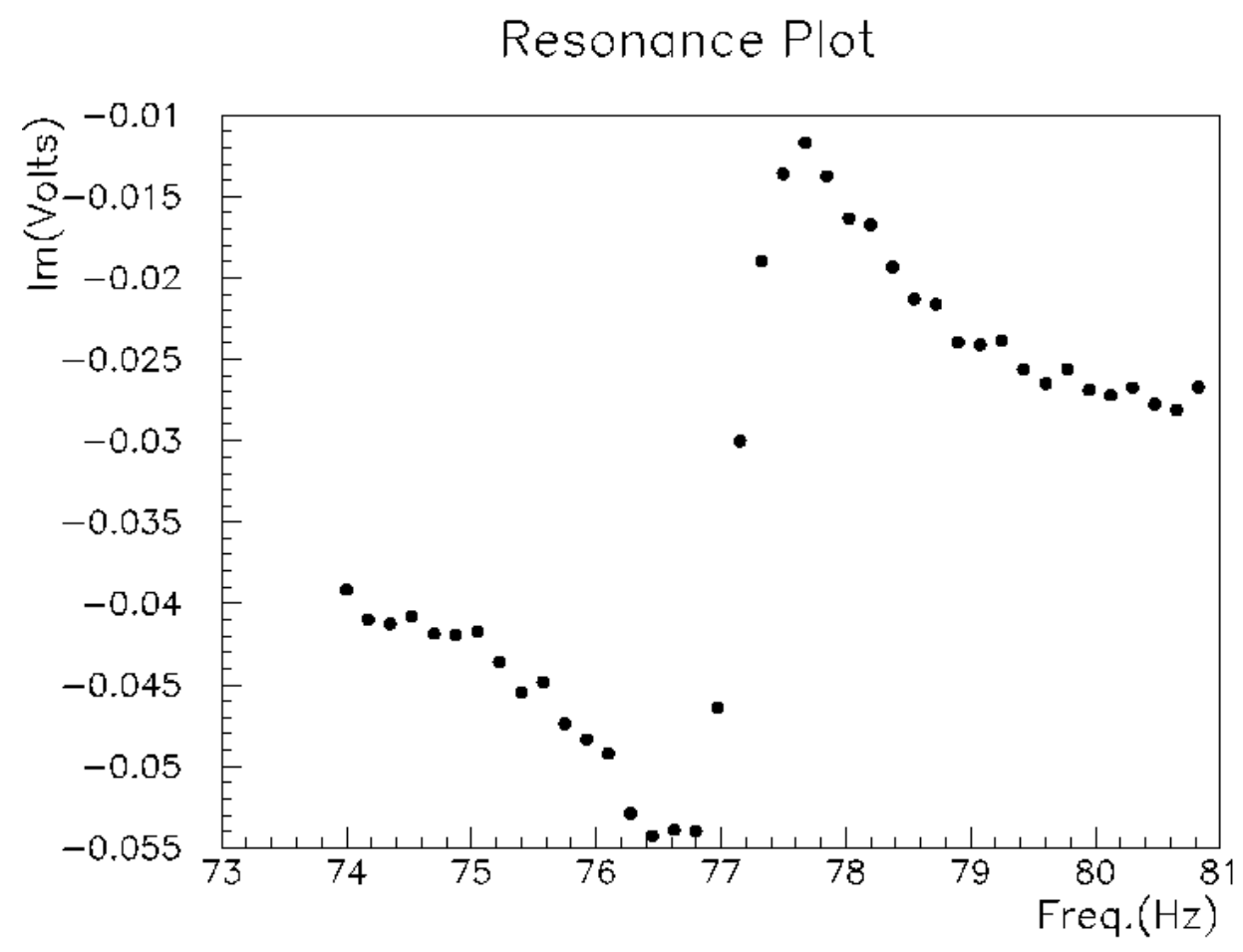

Figure B.2: A plot of the driving frequency versus the out-of-phase voltage.

problems between the wires and the connector pins on the end cards, 2 planes were rejected due to a wire-dependent tension that caused a large (9 gm) variation in the tension from one side of the plane to the other, and 9 planes were rejected due to other abnormalities (e.g., one or more wires with a tension so far from the nominal value that its resonance was outside of the frequency window).

\section{B.2 Dark Current Measurements}

After undergoing tension measurements, the wireplanes are installed in the highvoltage test boxes (shown in Figure B.4) for dark current ${ }^{3}$ and radioactive source testing. The purpose of these dark current measurements is to check the wireplanes for any defects that cause excessive current draw. In the lab, there are three boxes

\footnotetext{
${ }^{3}$ Dark current is the observation of anomalous current draw without ionization.
} 


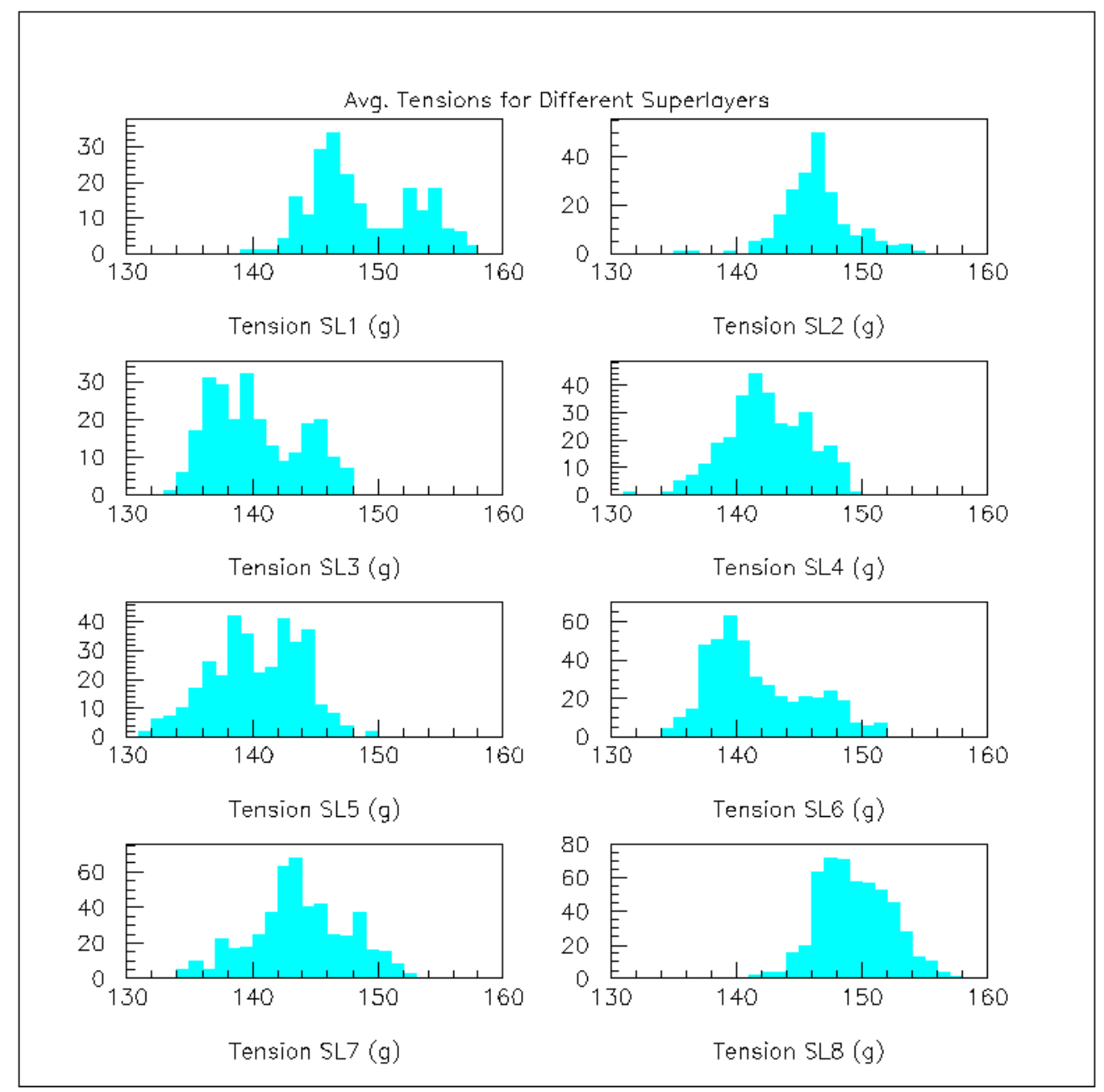

Figure B.3: The average tension (in grams) for each wireplane, plotted by superlayer number. 


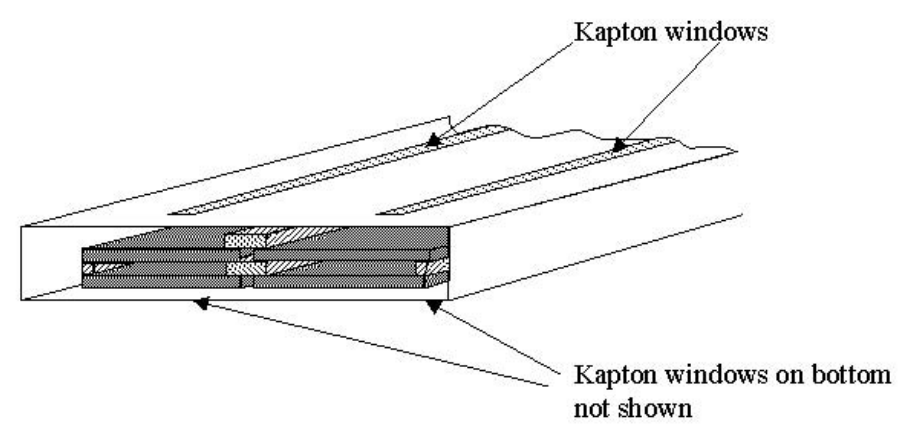

Figure B.4: A schematic of the four-wireplane high-voltage test box.

which are capable of holding up to four wireplanes each. Dark current and source testing is done on 12 out of 21 of all wireplanes that undergo tension testing. Dark currents are measured on wireplanes enclosed inside the test boxes which contain one atmosphere of $50 \% / 50 \%$ Ar- $\mathrm{C}_{2} \mathrm{H}_{6}$, bubbled through an isopropyl alcohol bath at $0^{\circ}$ C.

Electrostatics calculations were carried out for the drift-cell geometry of the test box to find the sense and potential wire voltages that give approximately the same field at the wires as for the COT geometry. In the test box setup, the nominal voltage settings for these fields was found to be $3.3 \mathrm{kV}$ and $2.6 \mathrm{kV}$ for the sense and potential wires, respectively.

An independent sense wire high-voltage supply is used for each of the four wireplanes in a test box, however all four wireplanes share a single high-voltage supply for the field-shaping wires. The voltages are delivered to each wire through a $100 \mathrm{k} \Omega$ high-voltage series resistor mounted inside a distribution box. The current draw of the sense wires on each plane is taken from the current output of each anode high-voltage power supply, fed through an analog multiplexer into a digital voltmeter (DVM) with a GPIB $^{4}$ readout. All 12 wireplanes (four in each of the three test boxes) have dark

\footnotetext{
${ }^{4}$ GPIB stands for General Purpose Interface Bus (also known as Institute of Electrical and Elec-
} 


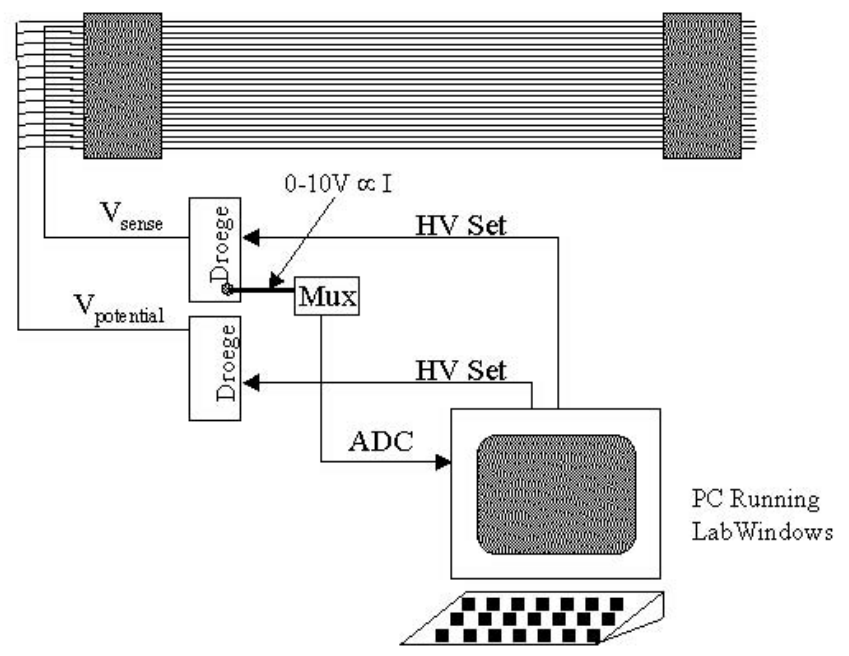

Figure B.5: A schematic of the data acquisition setup for dark current and source run testing.

current measurements performed on them at the same time, and the multiplexer cycles through feeding each plane to the DVM for readout. Figure B.5 is a schematic of the dark current data acquisition setup.

Due to a less than infinite resistance to ground in the distribution boxes and elsewhere in the setup, the typical current draw for a HV channel, without a wireplane connected, is on the order of $\sim 1 \mu \mathrm{A}$. In principle one can zero out this offset at the high-voltage supply itself, but we have found that the zero point tends to drift, presumably due to temperature and humidity effects. To circumvent this difficulty, we remove the $I=V / R$ component of the current draw by measuring the ratio of the dark current at the nominal voltage settings and with both sense and potential voltages set $200 \mathrm{~V}$ over nominal. The distribution of the dark current ratio $($ nominal +200$) /$ nominal is shown in Figure B.6. The ratios for nearly all of the wireplanes tested matched expectations. Only three wireplanes have been rejected due to tronic Engineers (IEEE) Standard 488). Hewlett Packard invented the GPIB bus in the late 1960's. GPIB was designed for connecting computers and instruments in a reliable manner, and possesses desirable features such as the remote control of instruments, data handshake for reliable operation, and the capability of real-time response. 


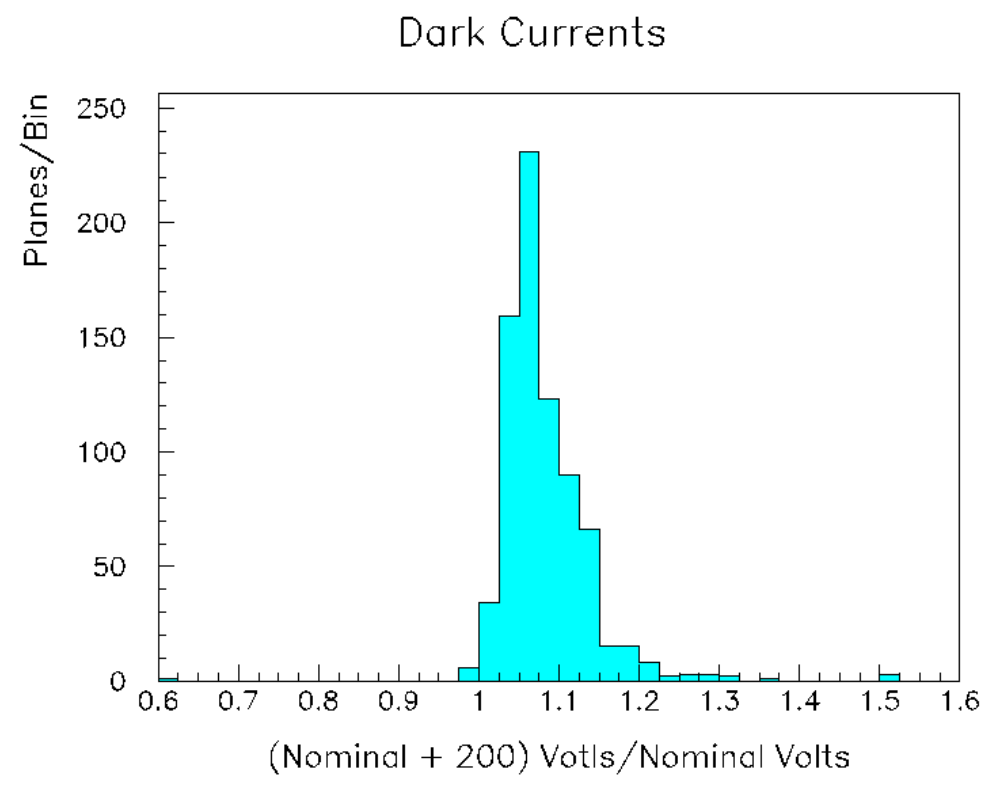

Figure B.6: The distribution of the ratio of the dark currents measured at nominal voltage (denominator) and nominal $+200 \mathrm{~V}$ (numerator).

abnormal ratios.

\section{B.3 Source Runs}

We subject the same 12 out of 21 wireplanes that are put through dark current testing to overnight source run testing. This source testing is used to check for the onset of glow mode ${ }^{5}$, as well as general stability of operation in a high-rate environment.

The same test boxes and data acquisition setup used for dark current testing are used during source runs (Figures B.4 and B.5). These test boxes have four Kapton [89] windows, one centered just above or below each of the four wireplanes housed inside the test box, running the entire $10 \mathrm{ft}$ length of each wireplane. For the two wireplanes in the top position, the windows are in the lid of the box which also forms the upper ground plane. So not to interrupt the ground plane, the inside of the window is covered with 3 mil thick $\mathrm{Cu}$ tape. For the two wireplanes in the bottom position, the

\footnotetext{
${ }^{5}$ Glow mode is a self-sustaining discharge.
} 
window is below the tray on which the wireplane is mounted. It is also worth noting that the bottom trays are open in the middle and covered with 2 mil thick stainless steel sheet to provide the ground plane, so that the amount of absorber between the sources and the drift volume is minimized. In a source run, four $1 \mathrm{mCi}{ }^{90} \mathrm{Sr}$ sources, one for each wireplane in the test box, are driven the entire length of the planes by a stepping motor mounted on each test box. During a typical run, the sources take $\sim 10$ hours to complete their trip from one end of the test box to the other. For the duration of the source testing, the current is monitored in the same fashion as during the dark current test runs. From Reference [90], the current at each wire is expected to follow the relation

$$
i=i_{0} \cdot\left(\frac{h^{2}}{h^{2}+r^{2}}\right)+b
$$

where $h$ is the height of the source above the wireplane, $r$ is the distance from the source measured transverse to the wireplane and $b$ is a constant. For a $2 \mathrm{mCi}{ }^{90} \mathrm{Sr}$ source and a similar source testing setup, $i_{0}$ was measured to be $286 \mathrm{nA} / \mathrm{cm}$ and $b$ was $16 \mathrm{nA} / \mathrm{cm} . b$ is assumed to be geometry dependent, and therefore not transferable to our chambers; we set $b=0$ (it's small in any case). For our $1 \mathrm{mCi}{ }^{90} \mathrm{Sr}$ sources, we use $i_{0}=143 \mathrm{nA} / \mathrm{cm}$, which gives about $11 \mathrm{nA} / \mathrm{cm}$ at the outside wires of the plane. This is the expected current on the innermost superlayers at design luminosity. At the center wire, just below the source, we get $143 \mathrm{nA} / \mathrm{cm}$, which is 10 times the design current. However, the total integrated charge is small. Using a current draw of $2 \mu \mathrm{A}$ on the center wire, which is one-half the measured current draw for a source twice the strength of ours [90], a 10 hour exposure time produces an integrated charge of $2 \times 10^{-4} \mathrm{C} / \mathrm{cm}$. This is to be compared to $6 \times 10^{-2} \mathrm{C} / \mathrm{cm}^{-\mathrm{fb}^{-1}}$ for the innermost layers of the COT at design luminosity. The $2 \mu \mathrm{A}$ current draw is consistent with what we measure during our source runs, but we measure all sense wires in a given plane together, not the individual wires.

Figure B.7 shows a typical plot of current measurements for a 9.5 hour source 


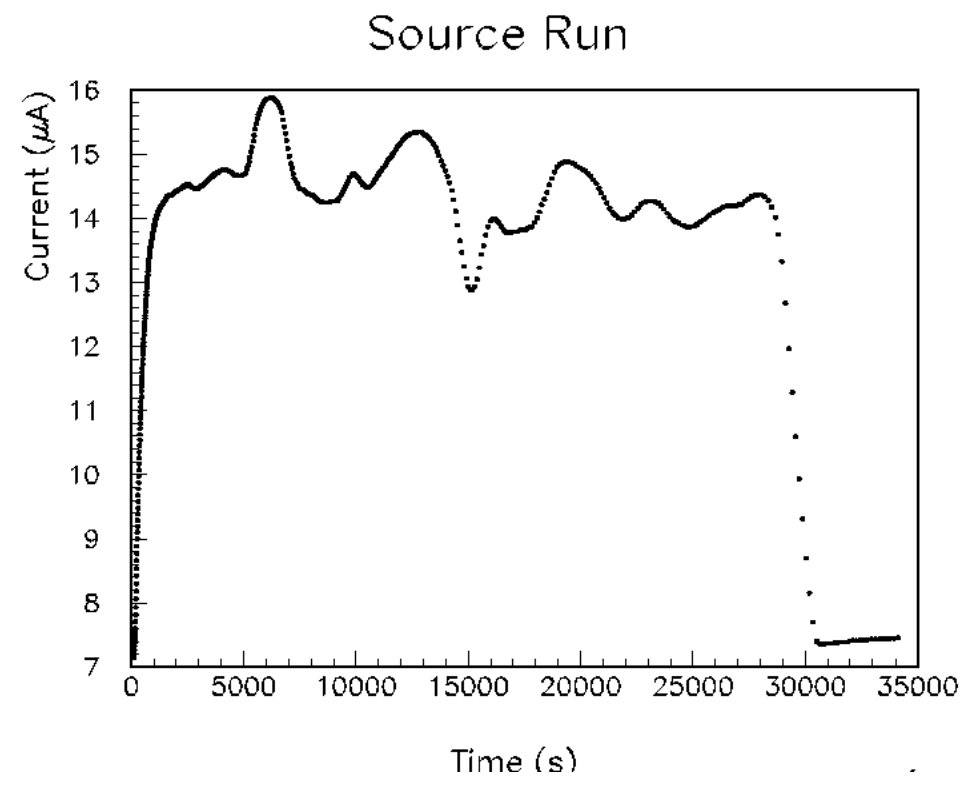

Figure B.7: Current measurements for one wireplane during a 9.5 hour source run.

run. Several features are apparent: The sharp turn on and turn off at either end are due to the start and finish of the source over the printed circuit end-cards of the wireplane. In the start and finish positions, the illumination of the plane by the source is approximately one-half of the illumination when the source is well away from the edges (note the suppressed zero in the plot). Through the remainder of the plot, one observes a dip at the center, due to the PVC wire support, and an otherwise a fairly bumpy distribution with a total current of about $14.5 \pm 1.0 \mu \mathrm{A}$. The additional structure (beyond the wire support dip) is typical, and is due to local geometry (such as creases in the copper tape or Kapton covering the source windows).

Due to the large and varying current draw, the source run data are not terribly useful in looking for glow mode with a typical current draw of tenths of a microamp. Therefore, our glow mode tests are done as follows: At the end of the source run the sources are removed from the chambers while the high-voltage remains on. The currents on each wireplane are read out and then the high-voltage is cycled once and the currents read out again. The glow mode can be detected by comparing the 
currents before and after the high-voltage cycling. As proof of this, we noted that early source runs were carried out with pure $\mathrm{Ar}-\mathrm{C}_{2} \mathrm{H}_{6}$ and no isopropyl. Under these conditions glow was observed on most planes as a decrease of anywhere from 0.2$1.0 \mu \mathrm{A}$ in the current measured after cycling the high-voltage. We were frequently able to localize the glow, by exposure with the sources, and found it to occur at the connector pins at the far (non-electronics) end of the plane, which in our source run setup are exposed (this is not the way it is in the COT). The addition of a small amount of isopropyl (bubbled through at $0^{\circ} \mathrm{C}$ ) to absorb UV photons eliminated the problem. Since that time we have detected glow mode on just 5 wireplanes, all these have been rejected. In addition, 4 wireplanes were rejected for failure to hold highvoltage through the duration of the source run, and one other was rejected due to an extremely abnormal current versus time plot. Finally, 15 wireplanes were damaged during handling or arrived damaged, and one wireplane was rejected due to a broken end-card. 


\section{Appendix $\mathrm{C}$}

\section{The Muon Calibration System}

This appendix describes the hardware and software for the calibration system at CDF in Run II for the CMP, CMX, BMU muon drift chambers as well as the associated scintillator systems. The CMP drift chambers were constructed at the University of Illinois. The muon calibration system for Run II was created and developed by the University of Illinois.

As mentioned in Section 2.2.3, muons are detected at CDF with the single-wire drift chambers (Figure C.1) when a charged particle traverses the chamber and ionizes the gas contained within the drift cell volume. The drift tubes are $2.54 \times 15.24 \mathrm{~cm}$ in cross section and are built in lengths ranging from 1.8 to 6.4 m made of aluminum extrusions with walls $0.26 \mathrm{~cm}$ thick and have a single $50 \mu \mathrm{m}$ gold-plated tungsten sense wire suspended in the chamber center [91]. Field shaping is done through the use of $14.5 \mathrm{~cm}$ wide Glasteel [92] shaping boards that are copper-clad on one side. The copper-clad surface is scored with a special cutting device to produce 17 evenlyspaced strips of copper. These cathode strips are mounted on the inner top and bottom surfaces of the drift tube. A potential difference is created within the drift cell by placing high-voltage on the anode (gold-plated sense wire) of $5.6 \mathrm{kV}$, and on the cathode (copper strips which line the chamber walls) of $3.0 \mathrm{kV}$. When the gas inside the chamber (a 50\%/50\% mix of $\mathrm{Ar}_{-} \mathrm{C}_{2} \mathrm{H}_{6}$, bubbled through isopropal alcohol at $-7^{\circ} \mathrm{C}$ ) is ionized by a charged particle passing through the chamber volume, the 
freed electrons are accelerated toward the sense wire by this potential difference. The accumulation of charge on the anode wire results in electrical signals that are capable of being read out by chamber-end electronics. These signals are then amplified in two stages: once by the pre-amplifiers in an electronics package mounted on the drift chamber end, and eventually by an amplifier mounted on an amplifier-shaperdiscriminator (ASD) board. After the second stage of amplification, the ASD converts these signals into differential emitter-coupled-logic (ECL) and sends the signals from the collision hall, up to the counting room on the first floor of the B0 building. The conversion of the signals into differential ECL prior to their transmission up to the counting room is done so that any common-mode interference which is introduced into the signal lines on their way through the 200 -foot length of cable up to the counting room is cancelled out when the differential signals are summed back together. The digital signals output by the ASD are accepted by a VME-based 96-channel timeto-digital converter (TDC) board which resides in the B0 counting room. There is a 2000 ns window to record hit times and widths of ECL signals from the muon system ASD boards. The TDCs convert the ASD signals into drift times. Signals are read out of the TDCs by the CDF data acqusition system; one channel per wire and trigger hits are formed from coincidences of nearby wires. In the case of the CMP, these trigger hits are complemented by trigger information from the CMU chambers.

The muon scintillator systems detect muons through the use of fluor-doped plastics which emit light when a charged particle passes through the material. CSP sidewalls and the CSX are comprised of NE-114 scintillating material. The BSU, as well as the CSP top and bottom sections, are made from UPS-93-A scintillating material complemented with wavelength-shifting fiber bundles. The TSU (innerradius counters for the $\mathrm{BMU}$ ) are $\mathrm{BC}-408$ scintillating material also complemented with wavelength-shifting fibers. The TSU, as well as the BSU and the top and bottom sections of the CSP, are read out using control and concatenation units (CCUs) with 


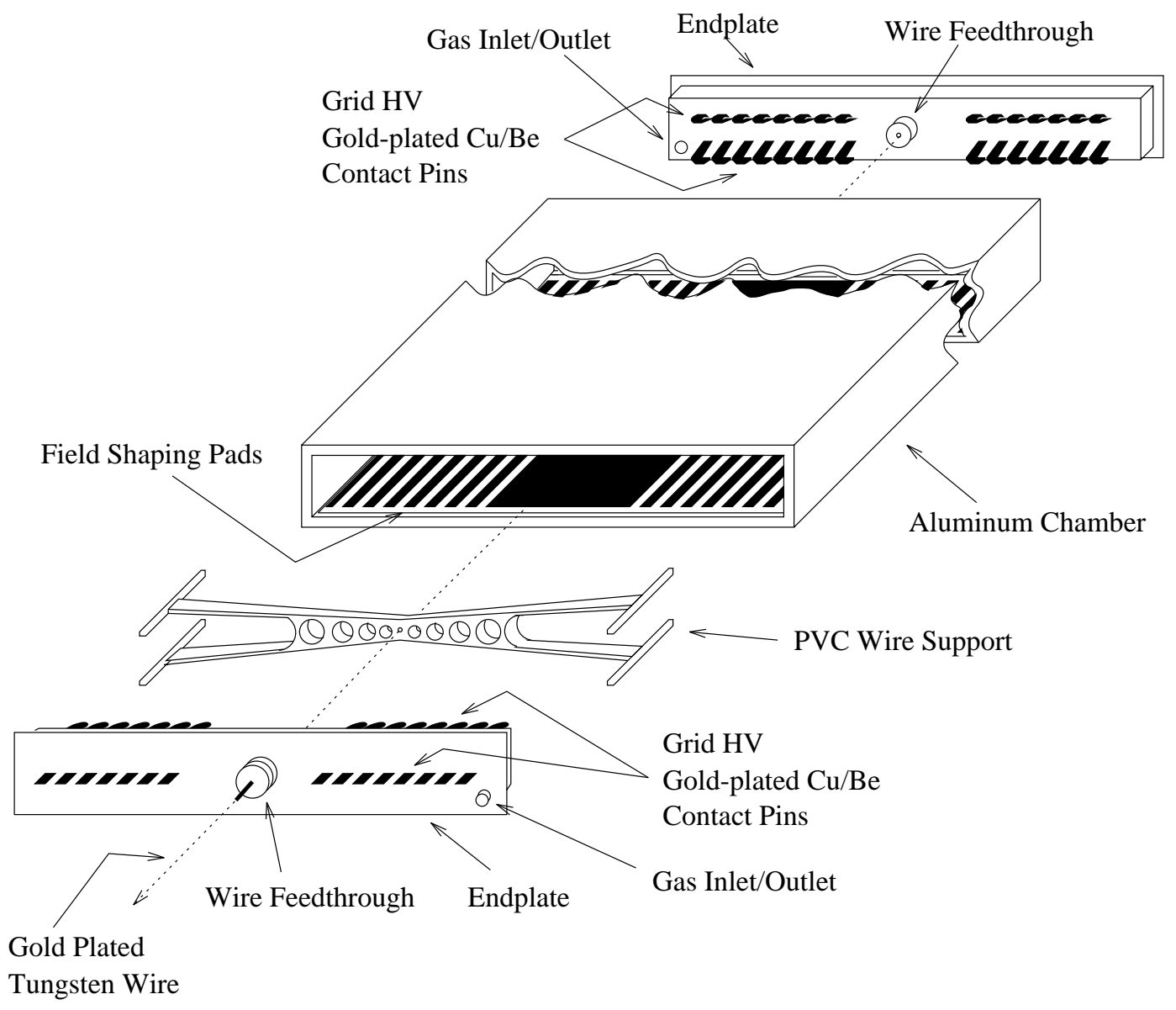

Figure C.1: A schematic view of a CMP/CMX drift chamber. Note that the PVC wire supports are used only for the CMP chambers.

light-collection done via H5783 photo-multiplier tubes (PMTs). The PMTs collect the scintillation light and convert it into electrical signals. The scintillator detectors have response times which are faster than those of the muon chambers that they are intended to complement (the drift times of the muon chambers is long compared to the spread of arrival times of the background particles). This faster response time makes the scintillator detectors particularly useful for reducing noise hits from chambers, as well as providing fast trigger information. The electronic signals from the PMTs are gated and digitized using discriminators and the logic signals are fed into the TDCs and eventually read out by the CDF data acquisition system.

The muon calibration system is intended to mimic signals in the drift chambers and 
the scintillator which are very similar to those produced from bona fide muons. These calibration signals are then read out through the usual data acquisition channels and provide system experts with feedback regarding the condition of the muon detector electronics. The calibration system is intended to monitor detector performance by alerting experts of any malfunctioning or dead channels which might develop over the course of time that the CDF experiment is in operation. However, this system has also proven to be particularly useful when experts are performing maintenance on a muon detector subsystem and would like to verify that a problem has been fixed.

\section{C.1 Chamber Calibration Hardware}

For the muon drift chambers, the calibration system mimics muon hits by injecting charge into the chamber-end preamplifier electronics.

At CDF, the Trigger Supervisor (TS) is a piece of hardware that coordinates the timing and readout of the entire detector in response to various trigger decisions. During calibrations, the TS controls calibrations by administering the timing of calibration input (e.g., the signal sent to preamplifiers) and coordinates them with the readout of calibration data. The TS talks to CDF detector hardware through the TRigger And Clock plus Event Readout (TRACER) board, which is VME-based. One TRACER in each VME crate accepts TS signals and passes all necessary information on to the rest of the crate electronics via the VME back-plane. The muon calibration hardware accepts the TRACER calibration signals and sends them to var-

ious non-VME based electronics which could reside in the CDF counting room (e.g., CAMAC-based modules) or down in the collision hall area (e.g., ASD boards).

The main component of the calibration system is the VME-based Digital Delay Generator (DDG). The DDG provides logic signals at programmable delay values and, at the request of a trigger signal, sends out a TTL timing pulse synchronized 
to the CDF clock. A VME-based calibration interface card (CIC), upon receipt of the appropriate calibration enable signal, generates a trigger for the DDG causing it to fire. In the chamber calibration setup (Figure C.2), pulses from the DDG are converted to differential ECL and fanned out using a customized VME-based fanout board. Signals from fanout card are sent down to twelve Redundant Analog Bus-Based Information Transfer (RABBIT) crates in the CDF collision hall. An ASD calibration board in the RABBIT crate fans out the calibration signal to each ASD card the crate. The ASD board discharges a capacitor and injects charge into the chamber-end preamplifier circuitry. Charge in preamplifier mimics the charge collected from an anode wire in the muon chamber and is read out just like data through the usual data acquisition chain. A VME-based board which speaks RS232 protocol is capable of communicating to the calibration cards in the RABBIT crates to manually select a specific sub-group of channels in that crate for calibration pulsing. The smallest selectable group of channels is half of an ASD board, or 24 channels. A VME-based analog-to-digital converter (ADC) is employed to monitor the voltage setting for charge injection into the preamplifiers.

\section{C.2 Scintillator Calibration Hardware}

For the newer Run II scintillator (BSU, TSU and CSP top and bottom) a muon signal is mimicked through a NIM timing pulse which triggers an LED mounted inside the scintillator panel, which is then collected by the PMT. In the older scintillator detectors, from the Run I era (like the CSX and the CSP side-walls), a NIM pulse flashes the scintillator discriminators and these signals are then read out with the TDCs. In most cases the calibration system provides verification of PMT functionality, as well as measurements to calibrate the PMT gain.

The same DDG module that is used for chamber calibrations is used to send 


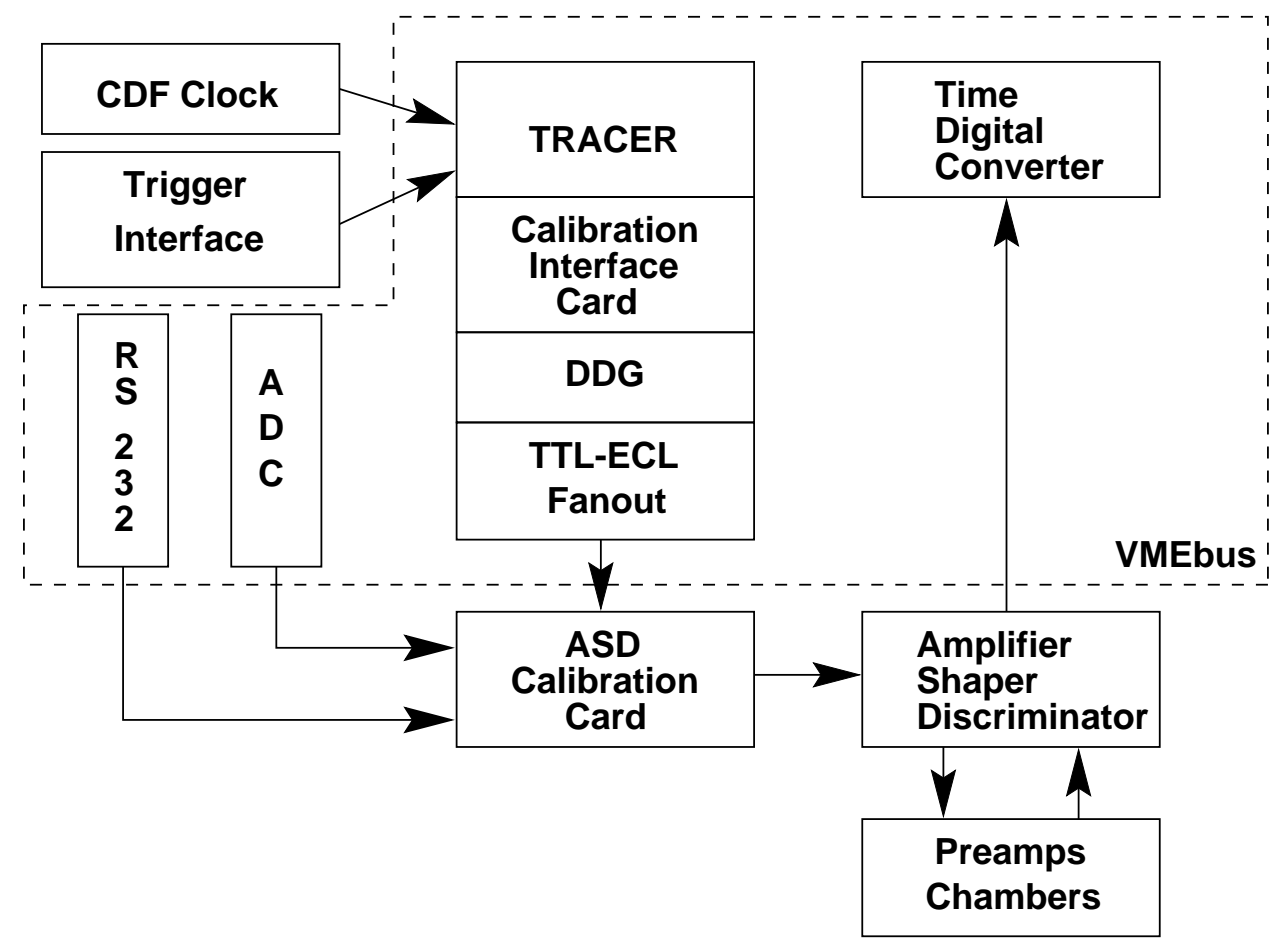

Figure C.2: The calibration hardware setup for the CDF II CMP, CMX and BMU drift chamber muon systems.

pulses on to the scintillator electronics. Here the TS sends commands to the nonVME electronics used for the scintillator readout. The DDG signals in the scintillator setup (Figure C.3) take two paths, depending on if the scintillator is the older Run I era detector or part of the newer Run II system.

The Run I era system is comprised of CSX scintillator and the CSP side-walls. These older systems send raw PMT signals to NIM-based discriminator modules. The digital output from these NIM modules are sent on to the TDC boards. Calibration signals from the DDG cause the discriminators to fire (no LEDs are embedded in plastic for the older scintillator detectors) and hence pass signals onto the TDCs.

The newer Run II scintillator is readout and calibrated using the CCUs. The top and bottom regions of the CSP, as well as the BSU and TSU scintillator systems collect scintillator light using PMTs which are then readout using the CCUs. The CCUs collect discriminated signals from the PMT Amplifier and Discriminators (PADs) 


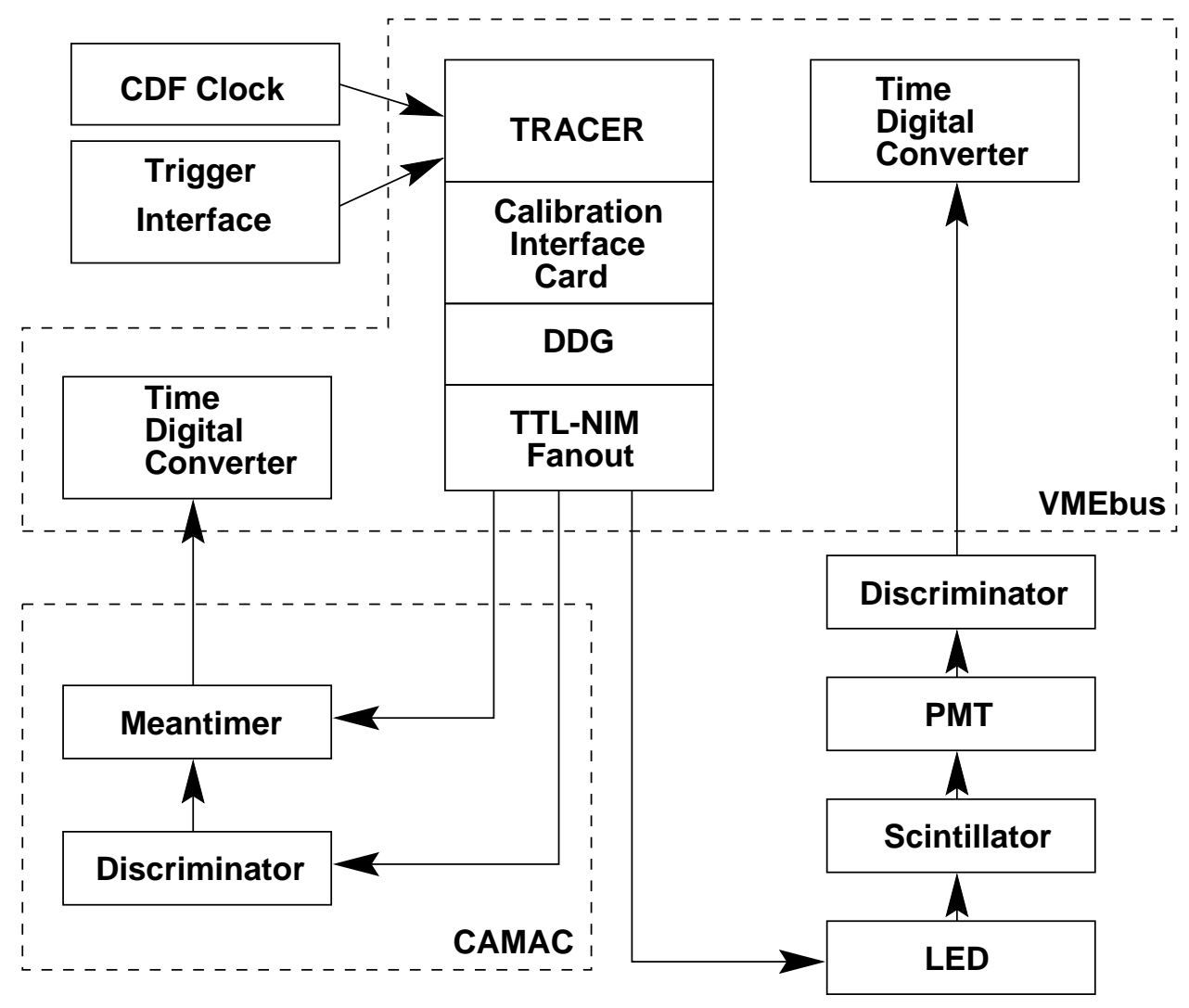

Figure C.3: The calibration hardware setup for the CDF II CSP, CSX, BSU and TSU scintillator muon systems.

and send these signals up to the TDCs. A LED embedded in the newer scintillator units can be made to flash by the CCU upon receiving calibration signals from the DDG. LED light is collected by the PAD and the readout is done exactly like real data, through the CCU and the TDC.

\section{C.3 Calibration System Software}

Much more detail on the calibration system software, including a discussion of system operation and performance is documented in [93]. This section will just briefly touch on software for the muon calibration system.

The system offers several standard modes of operation: continuous pulse, singledelay pulse, or multiple-delay pulse. In continuous pulse mode, the calibration system 
sends a steady and continuous stream of pulses with a specified and fixed period to the appropriate detector system. The continuous pulse mode is particularly useful to detector experts who are trying to narrow a problem down to one particular element in the muon detector readout chain. In the single-delay mode of operation, a set number of pulses (typically 1000) are sent by the calibration system to a specified muon subsystem. This mode of operation was used extensively during the commissioning of the calibration system; it can also be exploited to deduce different cable lengths used in the same subsystem by examination of the spread in hit times. Finally, with the multiple-delay pulse mode, the calibration system sends a set number of pulses at each of several delay values. Other than just checking to see if a particular detector is responsive by counting the number of hits recorded, the multiple-delay values allow experts to fit the distribution of hit times and delay values to verify a linear relationship between the two variables. 


\section{Appendix D}

\section{Signal Monte Carlo Event Generators}

During Run I, analyses carried out to measure $W$ boson polarization in top quark decay $[13,94]$ used a customized version of HERWIG [62, 63] (named "GGWIG") for signal modeling. We began exploring the idea of fixing the $W$ helicity using one of the latest Matrix-Element Monte Carlo (MEMC) generators, MadEvent [74, 75], in the hopes of fully exploiting MadGraph's calls to the HELicity Amplitudes Subroutines [95] (HELAS) library, where the helicity amplitudes of an arbitrary tree-level Feynman diagram are calculated. In principle it should be quite easy to fix the helicity of the $W$ boson using the MEMC generators, and at the time that this idea was under consideration none of the release versions of PYTHIA [66] or HERWIG could do this. ${ }^{1}$ Our desire to have a theorist-supported MC package which could fix the helicity of the $W$ boson in top decay was fulfilled thanks to the work of Tim Stelzer and Fabio Maltoni, who created a customized version of MadEvent named TT_DECAY. Having multiple MC generators which allow the user to fix the $W$ helicity will benefit us as experimentalists not only by bolstering our confidence in the precision of each generator, but also by aiding us in our systematics studies (one could imagine conducting pseudo-experiments about the signal distribution from one generator, and then fitting the "data" using the signal

\footnotetext{
${ }^{1}$ At the time of writing, the GGWIG code is rumored to be included in a pre-release version of HERWIG. Additionally, ALPGEN [69] has recently included the ability to fix the $W$ helicity in top decays.
} 
distribution from the second generator as a template).

TT_DECAY was spawned from MadEvent code for the process $p \bar{p} \rightarrow t \bar{t} \rightarrow b \bar{b} e^{+} v_{e} e^{-} \bar{v}_{e}$, and hence in actuality only generates events of this type (it will not directly generate events where the $W$ decayed hadronically, for example). The user can fix the $W^{+}$ boson helicity by setting the $i$ whel variable in the setcuts.f file to $-1,0$, or +1 . Since one must be in the top rest frame to fix the $W$ helicity to one of these values, it is not possible to set the helicity of both the $W^{+}$and $W^{-}$bosons in any single event (simply because one cannot be in both the $t$ and the $\bar{t}$ rest frames simultaneously). The current version of TT_DECAY only allows the user to fix the helicity of the $W^{+}$ boson.

Obviously a generator which produces dilepton events exclusively is of limited use, so I have written a Perl script which, in addition to breaking up the large .dat files ${ }^{2}$ generated by TT_DECAY into 2500 event chunks, also replaces the electron, positron and neutrino particle ID codes from a decaying $W$ with a realistic (standard model) mix of quarks and leptons. Electrons and muons are detected differently in the CDF experiment, so we desire to generate both of these leptons in our Monte Carlo samples. Hadronically decaying $W$ s are desired so that we may generate a fair amount of lepton-plus-jets events (in the experiment, approximately $30 \%$ of all $t \bar{t}$ events will have this decay signature).

The remainder of this appendix serves as an overview of the steps necessary for generating a large number of events with the TT_DECAY package, pushing these events through PYTHIA, converting the .stdhep files into the .hepg format, and ultimately getting these events run through the full CDF detector simulation.

\footnotetext{
2 ". dat" is simply the suffix on files that ar output by the MadEvent Monte Carlo event generator. The format of these events is also known as "Les Houches" format.
} 


\section{D.1 Changing the random number seed}

The TT_DECAY package was spawned from an early version of MadEvent which had a fixed random number seed programmed into the FORTRAN code. We need to select

a different random number seed each time we run the package; if this is not done each series of events generated will be completely identical. A Perl script was created which changes the random number seed each time TT_DECAY is run, thus ensuring that each set of events generated is unique.

After the user sets the appropriate flags in the runTT_DECAY.pl Perl script, the TT_DECAY Monte Carlo program will run creating $N$.dat files of generator-level events (enough to fill the request of the total number of events specified by the user in the script), while choosing a new random number seed for each .dat file generated. The helicity of the events generated is also specified by the user in the Perl script. Depending on the "quality" of the random number seed (some seeds are better than others in the sense that using them results in the generation of a greater number of events in the .dat output file), a run of the TT_DECAY program with that particular seed may generate anywhere from $\sim 10 \mathrm{k}$ to $\sim 1 \mathrm{M}$ unweighted events.

\section{D.2 Correcting for the systematic sampling of phase-space}

The early version of MadEvent was coded in such a way that it systematically sampled phase space. The set of all possible initial conditions for a dynamical system is referred to as phase space. One can imagine the simple case of a six-dimensional space consisting of a particle's position $x, y, z$ and velocity $\dot{x}, \dot{y}, \dot{z}$, with each variable taking one dimension in phase space. If we sample phase space in a systematic fashion while generating our events and then, for example, only use the first 100 out of $N \gg 100$ events which were generated, we would be introducing a significant bias 
into the model and therefore the analysis. To avoid the risk of introducing any such bias altogether, we shuffle the events prior to use.

Specifically, we run the script runRandomize.pl over the .dat files that are directly output from MadEvent as a result of executing the previous script. runRandomize.pl is a Perl script which uses the Fisher-Yates shuffling algorithm [96, 97] to randomize the events:

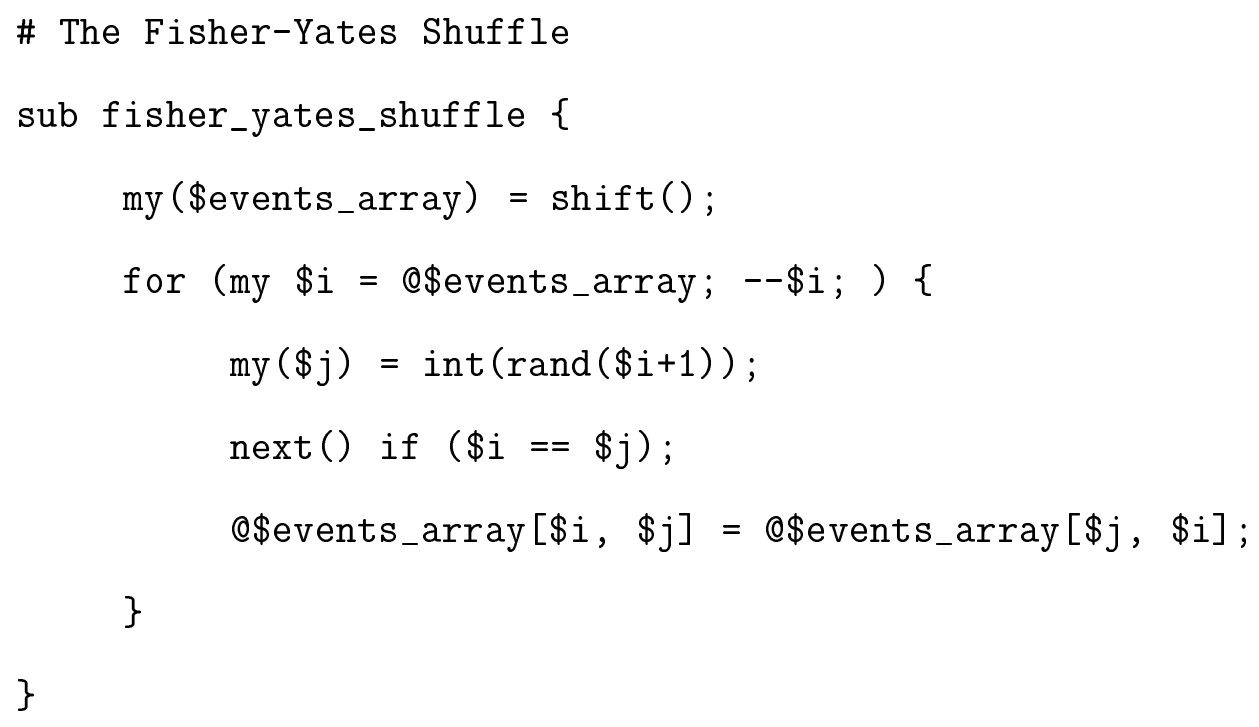

\section{D.3 Changing particle IDs}

As mentioned previously, the .dat files created by the TT_DECAY program only contain events where both $W$ bosons have decayed to electrons or positrons, plus neutrinos. The runRandomize.pl script, in addition to shuffling events, replaces the final-state particle ID codes in the generator-level files with a mix of light quarks and leptons. In instances where the script replaces lepton ID codes with those of light quarks, the appropriate color structure is also inserted into the Monte Carlo event record. A comparison of the kinematic information of light quarks and charged leptons from MadEvent $t \bar{t}$ semi-leptonic decays (where one of the $W$ bosons decays hadronically, i.e. to quarks) was carried out. At generator level, the four-momentum information 
from quarks or leptons in ttbar decays was found to be in agreement, so such a substitution is not as ad hoc as one might initially suspect. MadEvent treats the light quarks $(u, d$ and $s)$ as being massless. Additionally, this particular MC generator treats the charm, usually referred to as a heavier-flavor quark, as being massless. Such assumptions are appropriate, since the MadEvent Monte Carlo is used with cuts on the jet $E_{T}$ on the order of $5-10 \mathrm{GeV}$. In such instances dropping the charm mass leads to an error which is negligible.

The runRandomize.pl script concatenates all of the dat files generated for a particular helicity (which by now have had their events randomized) and breaks these events up into separate 2500-event files so that the Monte Carlo events are more easily managed by scripts to be applied at the later stages of the MC generation process. It is here that the events are re-numbered to be sequential once again.

\section{D.4 Adding parent particles}

The event record of the MadEvent .dat files include things like event number, particle ID, and 4-momentum information. Unfortunately, what the MadEvent output files do not explicitly include is the 4-momentum information for the intermediate parent particles (e.g., the 4-momenta of top quarks and $W$ bosons and the IDs for these parent particles). If one examines a raw MadEvent .dat file one finds that a final state electron, for example, will have an initial state parton ( $u$ or $d$ quark) as it's parent, not a W boson. Having the true parent particle information inserted directly into the event record has an advantage from the user's standpoint of adding the capability to distinguish an electron which came from the semi-leptonic decay of a top quark, from a swarm of electrons which came from the decays of unstable hadrons in the same event.

As a final task, the runRandomize.pl script sums the four-momenta information 
for daughter particles and inserts this energy and momentum information into the event record along with the IDs of the $t$ and $W$ parent particles.

\section{D.5 Fragmentation and hadronization}

Steve Mrenna has written up a very nice piece of FORTRAN code named "pyxtra" which reads in Les Houches formatted events from various MEMC generators and pushes those events through PYTHIA for fragmentation and hadronization [98].

PYTHIA uses a hadronization concept based on an older string model where the color-field lines are imagined as being concentrated into a tube between a coloranticolor source (like a $q \bar{q}$ pair). This "flux-tube" occurs because the gluons are self-coupling, and the field lines do not spread out. After the parton shower has ended, PYTHIA identifies the color-anticolor connections, and the strings (with energy and momentum) are pulled between them. The strings break into smaller bits, in accordance with some QCD dynamics programmed into the PYTHIA MC package, until these bits are small enough to be identified as physical particles (like pions, kaons, protons, etc.).

Another Perl script, run_pyxtra.pl has been written which pushes the .dat MadEvent Monte Carlo events through PYTHIA for fragmentation and hadronization. After passing through the PYTHIA software package, the events are stored in the .stdhep format.

\section{D.6 Conversion from .stdhep to .hepg}

Once the stdhep files generated by PYTHIA are in hand, we need to run production on them. Production is what we call the process of full detector simulation and event reconstruction using the offline software. Running production on the events preps them for offline analysis. Prior to running production on the PYTHIA output files, 
they need to be converted from the .stdhep to the .hepg format. Fortunately there is a module in the CDF offline software which is setup to do just that; it is part of the generatorMods package of cdfsoft2 and it is called hepevt2hepg.

Yet another Perl script has been written to help with running production on Monte Carlo signal events, it is called run_hepevt2hepg.pl and it runs the hepevt2hepg executable over all of the events in each of the . stdhep files converting them into the .hepg format.

\section{D.7 Detector simulation}

Once the .hepg formatted Monte Carlo files are in hand, full detector simulation can be run on the events. CDF is simulated using the GEANT3 [70] detector description and simulation tool. The CDF offline environment includes routines for carrying out

production on Monte Carlo events, where the events are pushed through the full detector simulation and then processed using the offline software. 


\section{Appendix E}

\section{The Mistag Matrix}

As discussed in Chapter 5, an important part of any analysis that relies on $b$-quark tagging is determining the background due to tagging light-quark or gluonic jets. This background can be estimated by using the tagging rates measured in an inclusive sample of generic jets from detector data. These datasets are collected from one of the following generic jet trigger-paths: JET_20, JET_50, JET_70, JET_100. The positive (negative) tag rate is important for the so-called "Method I" ("Method II"2) background calculation [99]. A positive (negative) tag is defined as a jet with a positive (negative) transverse distance from the primary vertex to the secondary vertex relative to the jet axis (known as $L_{x y}$, or the transverse decay length; see Figure E.1). Unlike positive tags, some fraction of which are due to heavy-flavor jets (from $c$ and $b$-quarks) even in the generic jet samples, the majority of negative tags in inclusive jets originate from light-quark jets (from $u, d$ or $s$-quarks). By knowing the tag-rate probabilities, one can calculate the expected number of mis-tagged lightjets in the $W+$ jet Monte Carlo samples by summing over the mistag probabilities for all of the jets in that particular sample. Detector simulation in Monte Carlo is capable of accurately reproducing the tag-rates for heavy-flavor jets, however not for

\footnotetext{
${ }^{1}$ In Method I we assume that the fake rate (i.e., tags with no heavy flavor) from generic jets is the same as those from $\mathrm{W}+4$ jets dataset. We then need to determine the tagging rate, $R_{t a g}\left(\Sigma E_{T}^{\text {jet }}, N_{t r k}^{\text {good }}, E_{T}, \eta, \phi\right)$, from the generic jets dataset and build the "fake tag-rate matrix".

${ }^{2}$ Instead of assuming that Method I is correct, one examines the transverse decay length distribution, $L_{x y}$, for secondary verticies. For negative $L_{x y}$ assume a very small contribution from heavy-flavor. Positive $L_{x y}$ is non-Gaussian due to the contributions from heavy-flavor.
} 


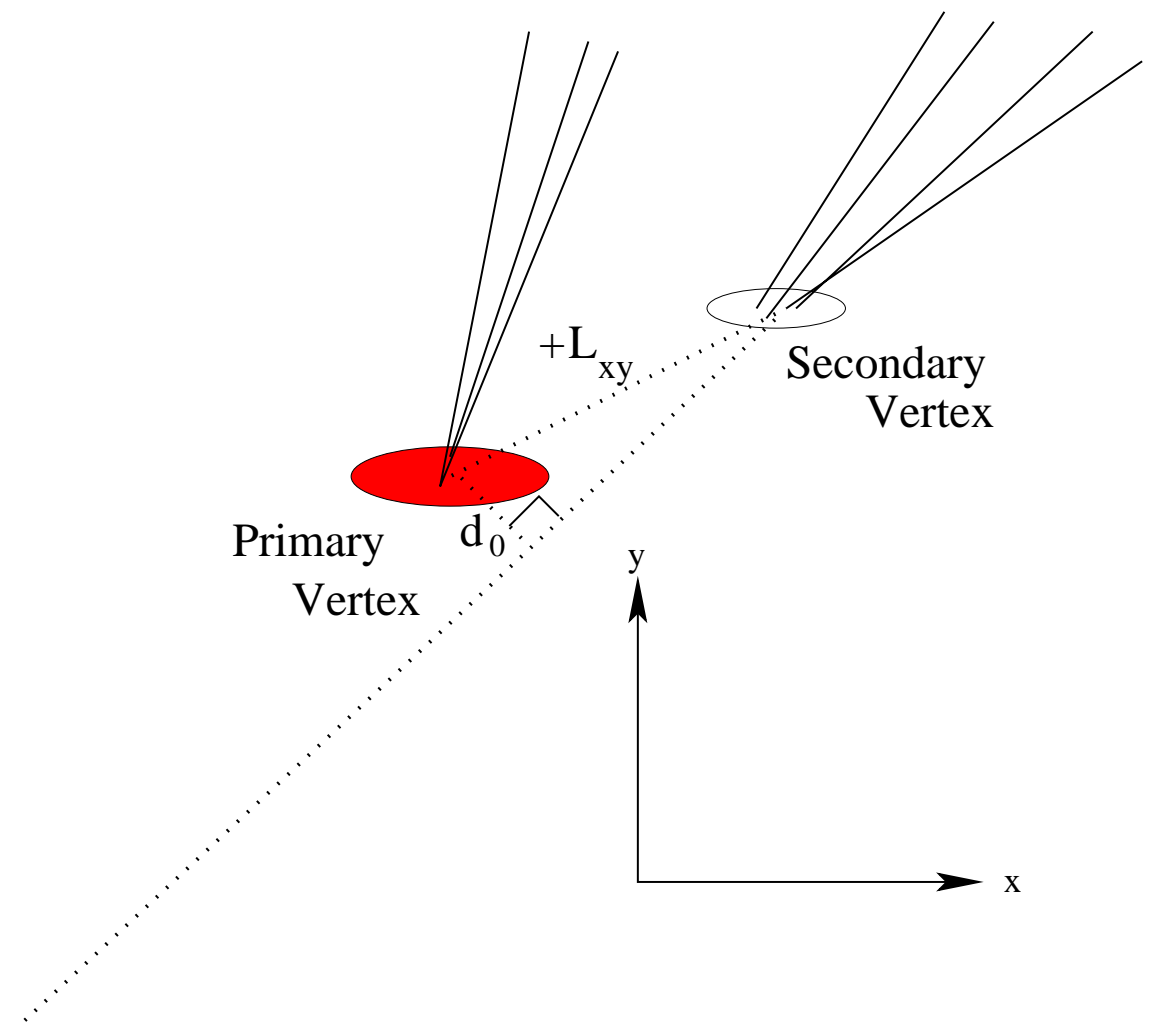

Figure E.1: $+L_{x y}$ is the distance between the primary vertex and the secondary vertex. The impact parameter, $d_{0}$, is the distance of closest approach of a track to the primary vertex.

the light-quark jets (which is known as the mistag rate).

\section{E.1 Data Samples}

The data used for determining and cross-checking the fake tag-rate matrix are based primarily on the secondary datasets skimmed and produced by the QCD working group at CDF. The data includes runs taken during the period between February 9, 2002 and November 4, 2002, with a total luminosity ${ }^{3}$ of $\sim 38.4 \mathrm{pb}^{-1}$. Only "good" runs were selected, thus requiring good status bits for the CLC, SVX, ISL, COT, HAD and EM calorimetry, SMX, CMU, CMP, CMX, and the triggers. The QCD

\footnotetext{
${ }^{3}$ The SecVtx tag-rate matrix has since been re-calculated using $110 \mathrm{pb}^{-1}$ of data. The $110 \mathrm{pb}^{-1}$ tag-rate matrix is used for the analysis described in this thesis.
} 
secondary datasets were reprocessed offline using the CDF 4.8.4 software release and the 4.9.1 b-tagging prescription.

\section{E.2 The Secondary Vertex Tagger}

The tagger that is used to create the mistag rate matrix and to carry out cross-checks is SecVtx. This tagger is described in more detail elsewhere [44], here I only note a few important details. The jets used by the SecVtx tagger are found using the JetClu jet-clustering algorithm with a cone size of 0.4 . The $E_{T}$ of the jets are corrected to the primary vertex on an event-by-event basis (because track $\theta$ depends on the $z$ position of the primary vertex). The SecVtx algorithm uses the run-averaged beamline with the z-position of the highest $\Sigma p_{T}$ vertex from the ZVertexColl collection as the reference vertex for any given event. The beam-line width is taken to be 32 $\mu \mathrm{m}$, independent of $z$, and the beam-line position is assumed to be stable within one run. All tracks are required to pass stringent track quality cuts (as discussed in Section E.3). The jet energies are not corrected for calorimeter response.

\section{E.3 Five-Dimensional Tag Rate Matrix}

The tag-rate matrix is binned in five parameters for both negative and positive tagrates, and hence is five-dimensional. Table E.1 lists the binning of the matrix in terms of these five variables. The positive, $R_{t a g,+}$, and negative, $R_{t a g,-}$, tag-rates are defined as follows for each bin $i j k l m$ in the space of $\left\{E_{T}, N_{t r k}^{\text {good }}, \Sigma E_{T}^{j e t s}, \eta, \phi\right\}$ :

$$
\begin{aligned}
& R_{t a g,+} \equiv \frac{\# \text { tagged jets with } L_{x y}>0 \text { in bin } i j k l m}{\# \text { taggable jets in bin } i j k l m} \\
& R_{t a g,-} \equiv \frac{\# \text { tagged jets with } L_{x y}<0 \text { in bin } i j k l m}{\# \text { taggable jets in bin } i j k l m}
\end{aligned}
$$



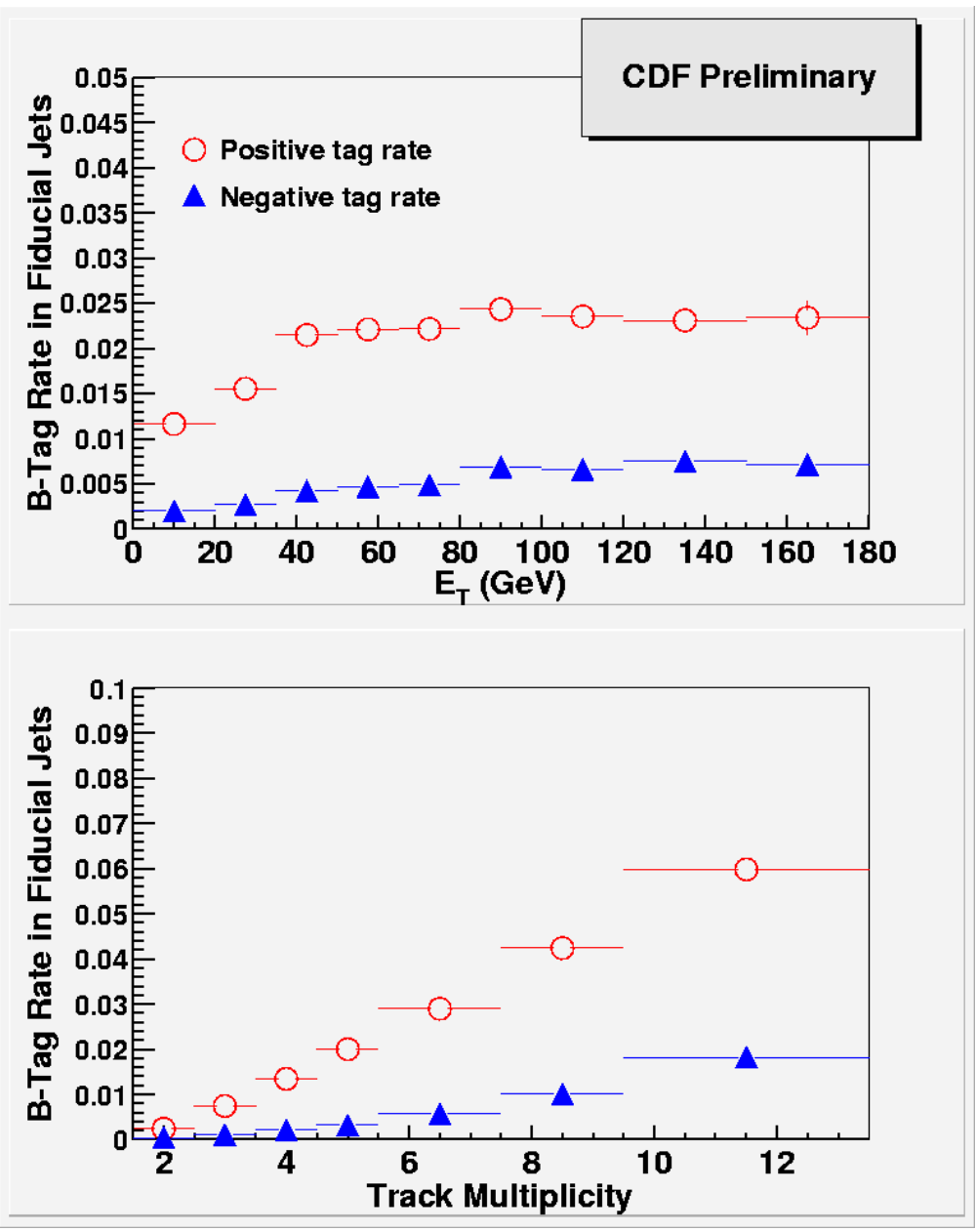

Figure E.2: Positive and negative tag rates in the combined jet sample as a function of $E_{T}$ (top) and taggable track multiplicity (bottom).

where $L_{x y}$ is the distance in the plane transverse to the beam along or against the direction of the jet from the reference point of the highest $\Sigma p_{T}$ primary vertex. $E_{T}$ is the transverse energy of the jet, $\Sigma E_{T}^{\text {jets }}$ is determined by summing over the transverse energies of the jets found using the JetClu jet-clustering algorithm with a cone size of $0.4, \eta$ and $\phi$ refer to the $\eta$ and $\phi$ of the jet, and $N_{t r k}^{g o o d}$ is the number of good tracks in the event.

A jet is defined as taggable if it passes the following requirements:

- Uncorrected $E_{T}>15 \mathrm{GeV}$ 


\begin{tabular}{|c||c|c|c|c|c|}
\hline \hline Bin & $E_{T}[\mathrm{GeV}]$ & $N_{\text {trk }}^{\text {good }}$ & $\sum E_{T}^{\text {jets }}[\mathrm{GeV}]$ & $\eta$ & $\phi[\mathrm{rad}]$ \\
\hline 1 & 0 & 2 & 0 & -2.4 & 0 \\
2 & 20 & 3 & 80 & -1.1 & 0.8 \\
3 & 35 & 4 & $\geq 140$ & -0.8 & 1.6 \\
4 & 50 & 5 & & -0.6 & 2.4 \\
5 & 65 & $6-7$ & & -0.4 & 3.2 \\
6 & 80 & $8-9$ & & -0.2 & 4.0 \\
7 & 100 & $10-13$ & & 0 & 4.8 \\
8 & 120 & $\geq 14$ & & 0.2 & 5.6 \\
9 & 150 & & & 0.4 & 6.4 \\
10 & $\geq 180$ & & & 0.6 & \\
11 & & & & 0.8 & \\
12 & & & & 1.1 & \\
13 & & & & & \\
\hline \hline
\end{tabular}

Table E.1: Tag rate matrix variables and bin boundries. The numbers shown are the lower bin boundaries.

- $|\eta|<2.4$

- $N_{t r k}^{\text {good }} \geq 2$.

The full five-dimensional matrices for $R_{t a g,+}$ and $R_{t a g,-}$ was created using even event numbers from the inclusive jet data. For the cross-checks, we use the statistically independent sample comprised of odd-numbered events. Figure E.2 shows the integrated tag rates for the combined QCD sample (even event numbers only). The upper plot shows the positive and negative tag rates as a function of $E_{T}$, integrated over $\Sigma E_{T}$ and track multiplicity; and the lower plot shows the rates as a function of the track multiplicity, integrated over $E_{T}$ and $\Sigma E_{T}$. The positive (negative) tag-rate rises from $1.2 \%(0.1 \%)$ at low $E_{T}$ to about $2.3 \%(0.6 \%)$ at high $E_{T}$.

The tag-rate matrix has been made available to users offline via an interface called using the CDF AC++ framework. 


\section{E.4 Cross-checks on the Tag-Rate Matrix}

We have performed a number of cross-checks of the tag-rate matrix. The general idea is to use the matrix to predict a certain number of tags for any particular bin. That is, for a given bin in $E_{T}$ (bin $i$ ), $\Sigma E_{T}^{\text {jets }}$ (bin $j$ ), number of tracks (bin $k$ ), $\eta$ (bin $l$ ), and $\phi$ (bin $m)$, where the number of taggable jets is (\# taggable) ${ }_{i j k l m}$, then the number of tags will be

$$
\begin{aligned}
& (\text { predicted pos. tags })_{i j k l m}=(\# \text { taggable jets })_{i j k l m} \times(\text { pos. tag rate })_{i j k l m} \\
& (\text { predicted neg. tags })_{i j k l m}=(\# \text { taggable jets })_{i j k l m} \times(\text { neg. tag rate })_{i j k l m}
\end{aligned}
$$

where the positive or negative tag-rate (for the bin $i j k l m$ ) is obtained from the appropriate tag-rate matrix. We compared both the number of predicted and observed tags

and predicted and observed tagging rates (obtained from Equations E.3 and E.4 by dividing the number of tags by the number of taggable jets) in bins of quantities that are not used to parameterize the tag-rate matrix, such as: run number, instantaneous luminosity, number of primary vertices, etc.

We also compare the overall number of predicated and observed tags. Good agreement is expected if the tag-rate matrix is accurate and properly parameterized. We performed this comparison in bins of quantities such as $E_{T}, \Sigma E_{T}, N_{\text {vert }}$, run number, instantaneous luminosity, etc. We calculate the error on the predicted number of tags as follows:

$$
\sigma_{\mathrm{tags}}=\sum_{i j k l m} \sqrt{(\# \text { taggable })_{i j k l m}^{2} \cdot \sigma_{\mathrm{rate}, i j k l m}^{2}}
$$

where $\sigma_{\text {rate, } i j k l m}$ is the uncertainty on the tagging rate for the bin $i j k l m$ from the mistag matrix. That is, we assume that the bin-by-bin errors are uncorrelated.

As was mentioned in Section E.3, we use orthogonal data samples for calculating and cross-checking the matrix. Moreover, we use odd event numbers in the JET_20, 
JET_50, JET_70, and JET_100 samples to count the number of observed tags and compare that with the number of predicted tags obtained using the even event numbers in the combined jet sample. We also use a sample collected through the $\Sigma E_{T}$ triggerpath (four jets with $E_{T}>15$ and $\Sigma E_{T}>125 \mathrm{GeV}$ ), which provides perhaps the best cross-check by virtue of being a completely independent dataset. Figures E.3E.7, at the very end of this appendix, show some of the comparison plots from these cross-checks. It is apparent from all of the plots that we have examined during our cross-checks that the number of predicted and observed tags generally agree within $1-2 \sigma$.

Finally, we carried out an exhaustive search for any biases introduced into the tagrate matrix due to trigger-jets. Trigger-jets are jets in an event with $E_{T}$ just above the threshold responsible for firing that particular trigger. For this series of tests, we used the standard tag-rate matrix described in Section E.3 to predict the number of expected tags, and we compare this prediction to the number of tags actually observed in the statistically independent (even numbered events) inclusive jet samples.

The trigger-jet was identified as the jet closest in $\eta$ - $\phi$ space to the Level-2 cluster just above the trigger threshold of the trigger type (e.g. JET_20 or JET_70) that selected the event. Tables E.2 and E.3 show the numerical comparison for both triggerjet and non-trigger-jet results, respectively. These results were obtained through selective cross-checking (i.e., running over trigger or non-trigger-jets) against the five-dimensional tag-rate matrix. In general, the ratios we observe, within statistical uncertainties, are $\sim 1$ for both trigger and non-trigger jets. We conclude that the trigger-jets do not introduce a bias into the matrix. 


\begin{tabular}{|c||c|c|}
\hline \hline \multirow{2}{*}{ sample } & positive tags (trigger jets) & negative tags (trigger jets) \\
\cline { 2 - 3 } & obs/pred & obs/pred \\
\hline \hline Jet20 & $1.01 \pm 0.02$ & $0.96 \pm 0.04$ \\
Jet50 & $1.01 \pm 0.02$ & $0.98 \pm 0.05$ \\
Jet70 & $1.01 \pm 0.03$ & $1.00 \pm 0.06$ \\
Jet100 & $1.03 \pm 0.03$ & $1.01 \pm 0.06$ \\
\hline \hline
\end{tabular}

Table E.2: Predicted vs. observed number of tags for trigger jets in each of the 4.8.4 (with 4.9 .1 b-tagging) datasets. This cross-check was done against the fivedimensional tag-rate matrix.

\begin{tabular}{|c||c|c|}
\hline \hline \multirow{2}{*}{ sample } & positive tags (non-trigger jets) & negative tags (non-trigger jets) \\
\cline { 2 - 3 } & obs/pred & obs/pred \\
\hline \hline Jet20 & $1.03 \pm 0.02$ & $1.01 \pm 0.06$ \\
Jet50 & $0.98 \pm 0.02$ & $1.03 \pm 0.05$ \\
Jet70 & $0.97 \pm 0.02$ & $1.01 \pm 0.05$ \\
Jet100 & $1.01 \pm 0.02$ & $1.01 \pm 0.04$ \\
\hline \hline
\end{tabular}

Table E.3: Predicted vs. observed number of tags for non-trigger jets in each of the 4.8.4 (with 4.9.1 b-tagging) datasets. This cross-check was done against the fivedimensional tag-rate matrix. 


\begin{tabular}{|c||c|c|}
\hline \hline \multirow{2}{*}{ sample } & positive tags & negative tags \\
\cline { 2 - 3 } & obs/pred & obs/pred \\
\hline \hline Jet20 & $1.01 \pm 0.01$ & $0.98 \pm 0.04$ \\
Jet50 & $0.99 \pm 0.01$ & $1.01 \pm 0.03$ \\
Jet70 & $0.98 \pm 0.02$ & $1.02 \pm 0.04$ \\
Jet100 & $1.01 \pm 0.02$ & $1.02 \pm 0.03$ \\
$\sum E_{T}$ & $1.02 \pm 0.01$ & $1.01 \pm 0.03$ \\
\hline \hline
\end{tabular}

Table E.4: Predicted vs. observed number of tags for the five-dimensional tag-rate matrix in each of the samples considered.

\section{E.5 Tag-Rate Matrix Systematic Uncertainties}

There are three major contributions to the systematic uncertainties in the fivedimensional tag rates:

- Biases for additional tag rate dependencies not included in the matrix

- Biases for runs not included when the matrix was created

- An uncorrected heavy-flavor contribution to the negative tag-rate

At the end of this appendix we show plots from some of the additional crosschecks of the five-dimensional tag-rate matrix against several variables which were not included in the matrix itself, but on which it might depend. These additional cross-checks were carried out using the $\Sigma E_{T}$ sample so that they could be done on a completely unbiased dataset. Recall that the $\Sigma E_{T}$ sample was not used during the determination of the matrix; because of high jet and track multiplicities this sample may be considered the worst possible case for the sake of such comparisons. Figures E.3-E.7 show that there are no systematic effects in the $\Sigma E_{T}$ cross-checks, and hence we take the statistical precision of these comparisons as our systematic uncertainty. The last row in Table E.4 shows that for the $\Sigma E_{T}$ dataset the statistical precision of our comparison is $1 \%$ for positive tags and $3 \%$ for negative tags. 


\begin{tabular}{|c||c|c|}
\hline \hline Systematic source & Positive tags (\%) & Negative tags (\%) \\
\hline Additional tag rate dependencies & 1 & 3 \\
Change in run conditions & 6 & 5 \\
HF contribution to neg. tag rate & - & 8 \\
\hline Total & 6 & 10 \\
\hline \hline
\end{tabular}

Table E.5: Systematic uncertainties for the Five-dimensional SecVtx tag-rate matrix.

The tag-rate matrix is determined using a subset of the goodrun list used for the 2003 winter conference analyses. Since we need to construct the matrix well in advance of the release of results in order to complete all of the systematics studies in time, the matrix is built on a subset of runs that do not extend to the end of the complete dataset. Due to a changing detector configuration, trigger and/or accelerator conditions, one has to worry whether the matrix adequately parameterizes the tag-rates in the newer data. When explicitly comparing the predicted to observed number of tags for the newer runs, we did not find any significant discrepancies, and we assigned the statistical precision of the tests, $\pm 6 \%$ ( $\pm 5 \%)$ for positive (negative) tags, as the systematic uncertainty due to these effects.

At present, the heavy-flavor contribution to the negative tag-rate is not corrected for in the five-dimensional matrix. The lack of such a correction means that the negative tag rate is systematically over-estimated. From the results of Run I studies carried out using a similar SecVtx tagger (Table 6 of [100]), we take the amount of this over-estimate to be about $8 \%$ for the negative tag-rate.

Table E.5 lists the major contributions to the systematic uncertainties for both positive and negative tags; the total is obtained by summing the individual contributions in quadrature. Based on our cross-checks, we take the systematic uncertainty on the five-dimensional matrix to be $\pm 6 \%$ for positive tags, and $\pm 10 \%$ for negative tags. 

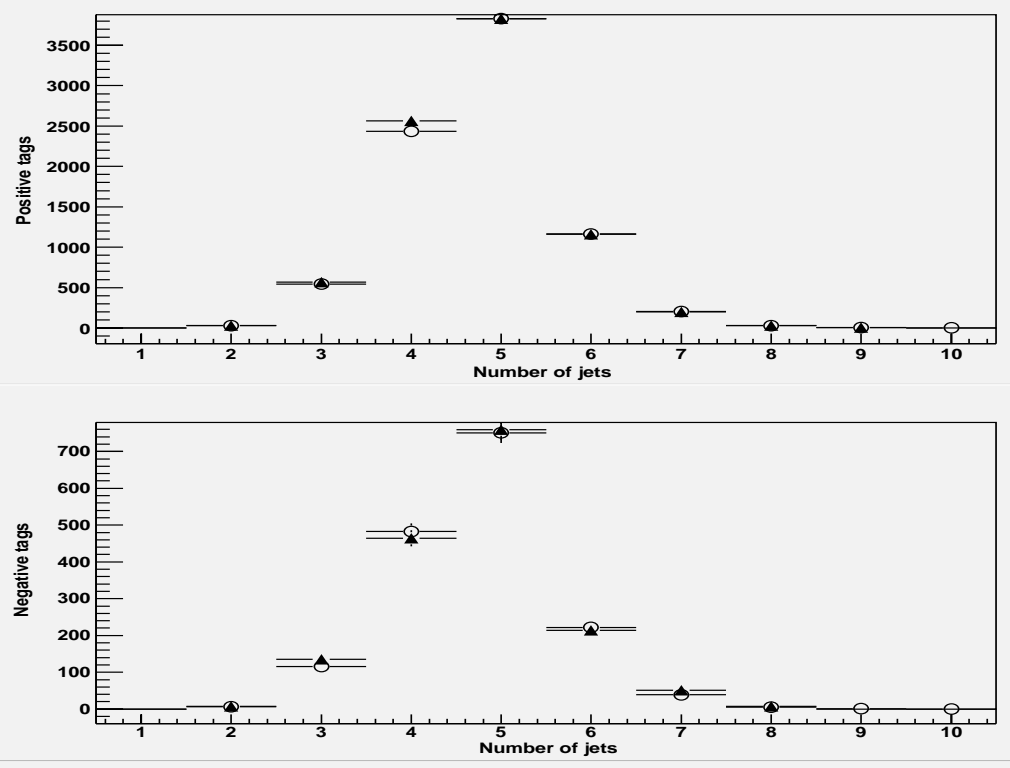

Figure E.3: Number of observed (triangle) and predicted (circle) positive and negative tags in the $\Sigma E_{T}$ sample binned as a function of the number of jets in the event.
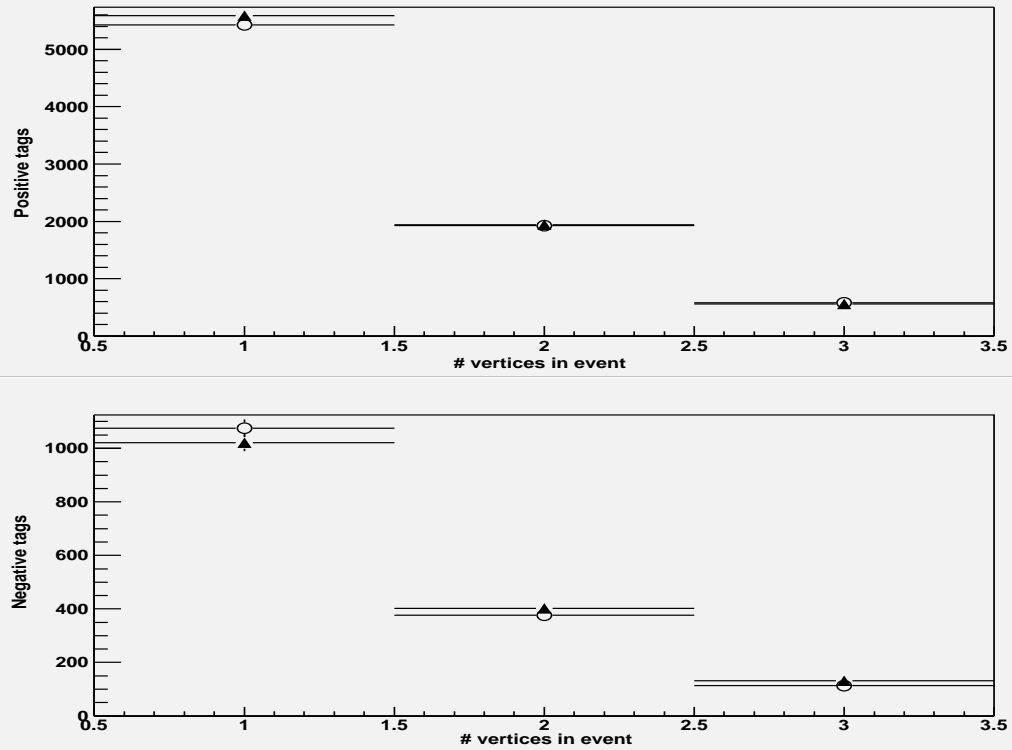

Figure E.4: Number of observed (triangle) and predicted (circle) positive and negative tags in the $\Sigma E_{T}$ sample binned as a function of the number of vertices in the event. 

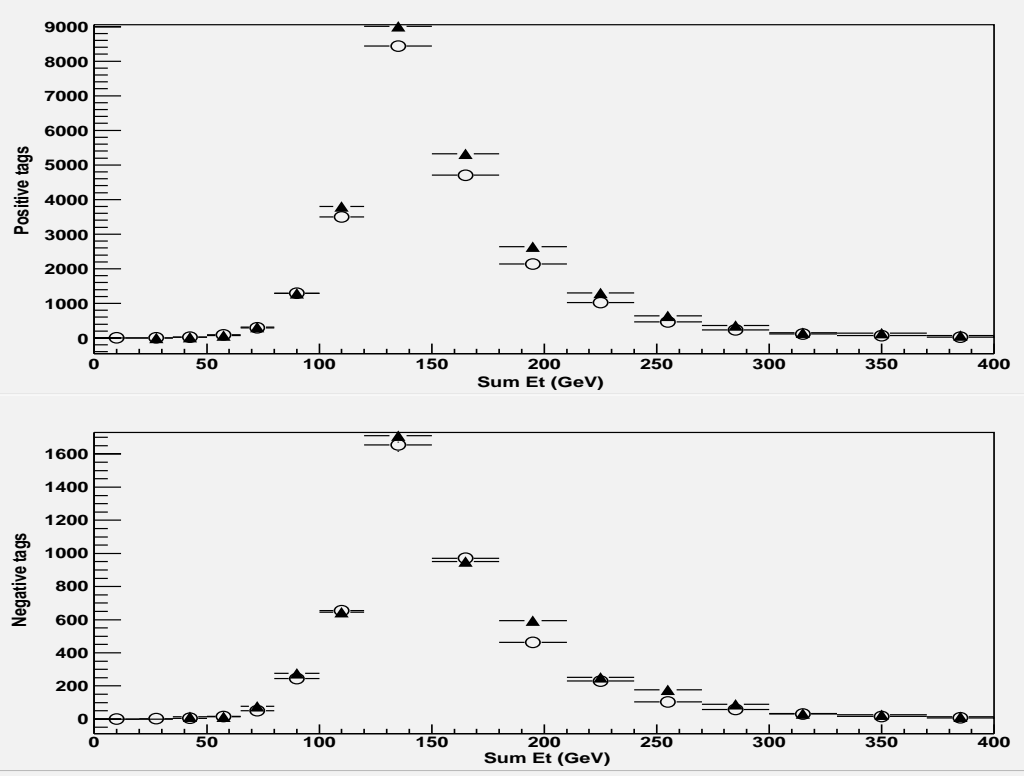

Figure E.5: Number of observed (triangle) and predicted (circle) positive and negative tags in the $\Sigma E_{T}$ sample binned as a function of the $\Sigma E_{T}$ of the event.
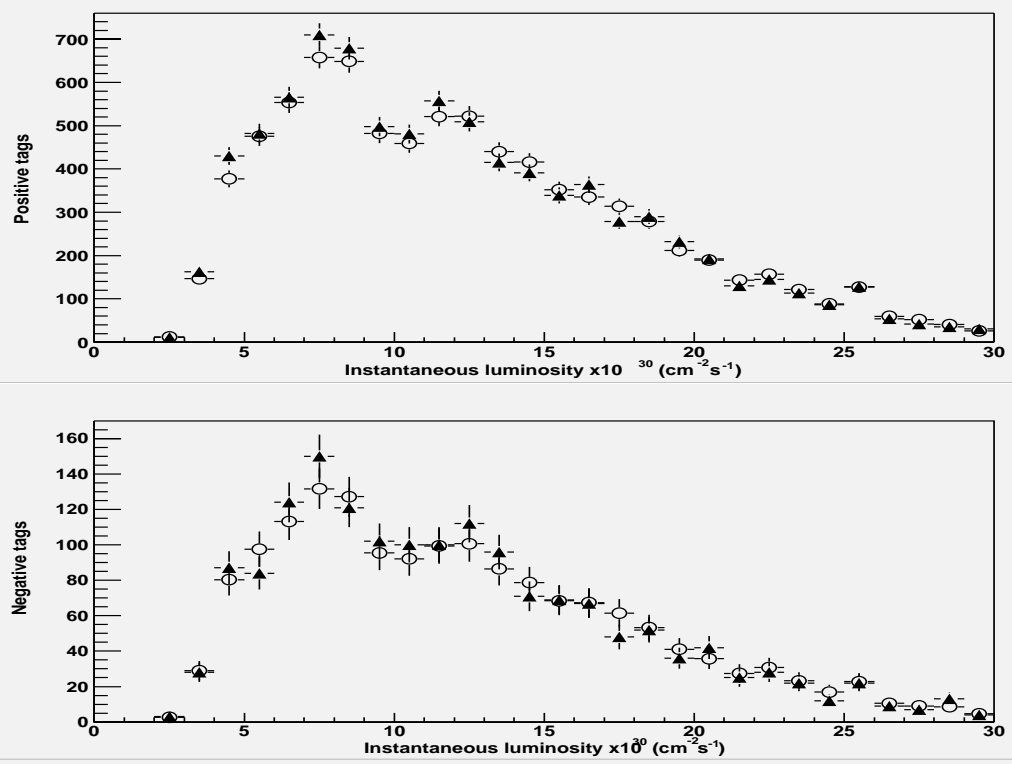

Figure E.6: Number of observed (triangle) and predicted (circle) positive and negative tags in the $\Sigma E_{T}$ sample binned as a function of instantaneous luminosity. 

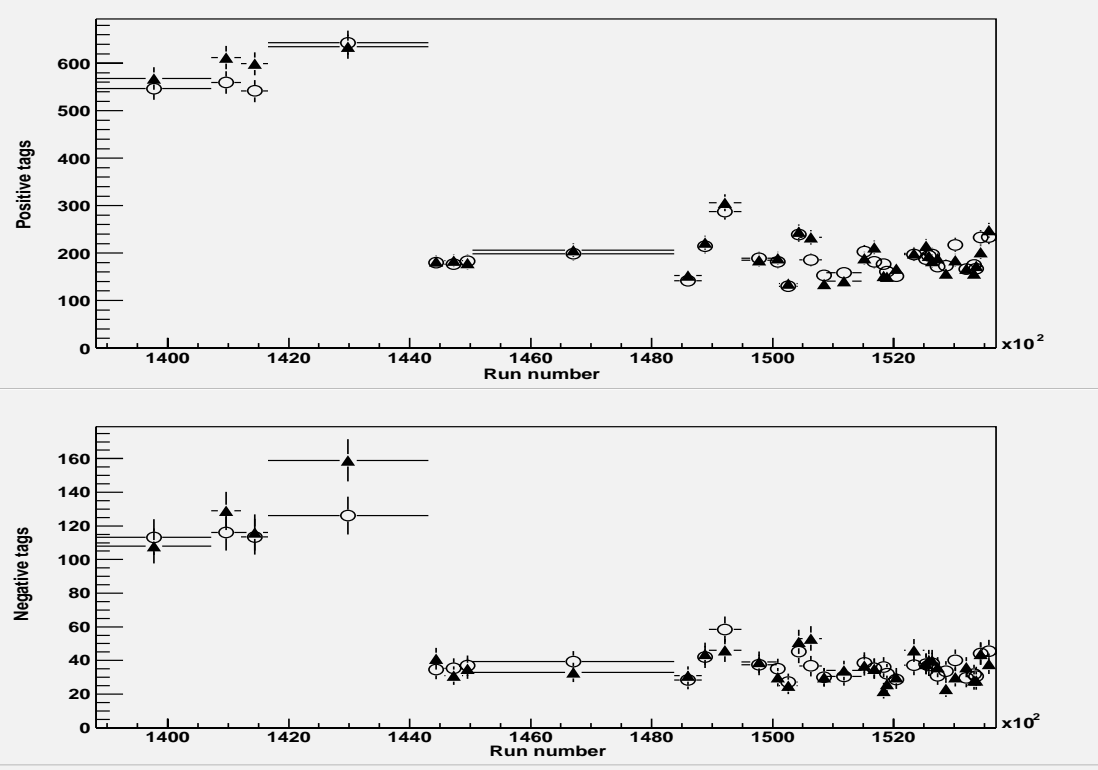

Figure E.7: Number of observed (triangle) and predicted (circle) positive and negative tags in the $\Sigma E_{T}$ sample binned as a function of run number. 


\section{References}

[1] There are many excellent texts describing the standard model of particle physics. Several which I find to be particularly helpful include:

F. Halzen and A.D. Martin, Quarks \& Leptons: An Introductory Course in Modern Particle Physics, John Wiley \& Sons, Inc. (1984);

C. Quigg, Gauge Theories of the Strong, Weak and Electromagnetic Interactions, Addison-Wesley (1997);

M.E. Peskin and D.V. Schroeder, An Introduction to Quantum Field Theory, Perseus Books (1995).

[2] Particle Data Group, Review of Particle Physics, Phys. Lett. B 592 (2004).

[3] CDF Collaboration, F. Abe et al., Phys. Rev. Lett. 74, 2626 (1995).

[4] D0 Collaboration, S. Abachi et al., Phys. Rev. Lett. 74, 2632 (1995).

[5] M. Beneke et al., "Top Quark Physics", CERN-TH-2000-100, (2000).

[6] N. Cabibbo, Phys. Rev. Lett. 10, 531 (1963).

[7] M. Kobayashi and T. Maskawa, Prog. Theor. Phys. 49, 652 (1973).

[8] G. Eilam, J. Hewett, and A. Soni, Phys. Rev. D44, 1473 (1991); Erratum, ibid. 59039901 (1999).

[9] CDF Collaboration, F. Abe et al., Phys. Rev. Lett. 80, 2525 (1998).

[10] G.L. Kane, G.A. Ladinsky, and C.-P. Yuan, Phys. Rev. D45, 124 (1992).

[11] E. Malkawi, C.-P. Yuan, Phys. Rev. D 50, R4462 (1994).

[12] J. Cao et al., "Supersymmetric effects in top quark decay into polarized $W$ boson", Phys. Rev. D68, 054019 (2003).

[13] CDF Collaboration, T. Affolder et al., Phys. Rev. Lett. 84216 (2000).

[14] D. Winn, Ph.D. thesis, University of Michigan, 2000 (unpublished).

[15] B. Kilminster, Ph.D. thesis, University of Rochester, 2003 (unpublished). 
[16] D. Acosta et al., "Measurement of V+A Fraction in Top Decay at CDF at sqrt(s)=1.8TeV", To be published in PRD RC.

[17] N. Goldschmidt, Ph.D. thesis, University of Michigan, 2004 (unpublished).

[18] J.D. Cockroft and E.T.S. Walton, Proc. Roy. Soc. (London), A136, 619 (1932), A137, 229 (1932), and A144. 704 (1934).

[19] http://www-bdnew.fnal.gov/operations/rookie_books/ Concepts_Rookie_Book_v3.pdf

[20] C. W. Schmidt and C. D. Curtis, "Operation of the Fermilab $\mathrm{H}^{-}$Magnetron Source." Proc. $4^{\text {th }}$ Int. Symp. on the Production and Neutralization of Negative Ions and Beams, Brookhaven, US, AIP Conf. Proc. No 158, 425 (1986).

[21] G. Ising, Ark. Math. Astron. Phys., 18, No. 30, Part 4, 45 (1925).

[22] R. Wideröe, Arch. Elektrotech., 21, 387 (1928).

[23] http://www-bdnew.fnal.gov/operations/rookie_books/LINAC_RB_v2.pdf

[24] K. Junck et al., "Commissioning and First Operational Experience of the 400MeV Linac at Fermilab." FERMILAB-Conf-94-181 (1994).

[25] http://www.fnal.gov/pub/inquiring/physics/accelerators/chainaccel.html

[26] http://www-bdnew.fnal.gov/operations/rookie_books/

Booster_Rookie_Book.pdf

[27] K. Wille, The Physics of Particle Accelerators: An Introduction, Oxford University Press (2000).

[28] http://www-bdnew.fnal.gov/operations/rookie_books/

Main_Injector_RB_v1.pdf

[29] http://www-bdnew.fnal.gov/pbar/documents/PBAR_Rookie_Book.PDF

[30] M. D. Church and J. P. Marriner, Ann. Rev. Nucl. Part. Sci. 43, 253 (1993).

[31] A.W. Chao and M. Tigner, Handbook of Accelerator Physics and Engineering, Second Edition, Section 7.2.12, World Scientific Publishing Co. (1999).

[32] A. Ruggiero, IEEE Trans. Nucl. Sci. 30, 2478 (1983).

[33] D. Mohl, G. Petrucci, L. Thorndahl and S. Van Der Meer, "Physics and Technique of Stochastic Cooling", Phys. Rept. 58, 73 (1980).

[34] B. Austin et al., IEEE Trans. Nucl. Sci. 30, 2593 (1983).

[35] A. Ando et al., IEEE Trans. Nucl. Sci. 30, 2031 (1983). 
[36] Image courtesy of Fermilab.

[37] M. G. Holloway and C. P. Baker, "Note on the origin of the term "barn"”, Los Alamos Research Report, LAMS 523 (1947).

[38] F. Abe et al., "The CDF II Technical Design Report", FERMILAB-Pub-96/390E (1996).

[39] C. Newman-Holmes, E.E. Schmidt, and R. Yamada, Nucl. Instrum. Meth. A 274, 443 (1989).

[40] A. Sill et al., Nucl. Instrum. Meth. A 447, 1 (2000).

[41] T. Affolder et al., Nucl. Instrum. Meth. A 526, 249 (2004).

[42] S. Rappoccio, J. Guimaraes da Costa, and K. Burkett, "First look at SecVtx Using Event Primary Vertex Finder PrimeVtx", CDF Internal Note 6417, 2003 (unpublished).

[43] K. Bloom and W. M. Yao, "' Outside-In" Silicon Tracking at CDF", CDF Internal Note 5991, 2002 (unpublished).

[44] D. Acosta et al., "Measurement of the $\mathrm{t}$ anti-t production cross section in $\mathrm{p}$ anti-p collisions at $\sqrt{s}=1.96 \mathrm{TeV}$ using lepton + jets events with secondary vertex b-tagging", hep-ex/0410041.

[45] L. Balka et al., Nucl. Instrum. Meth. A 267, 272 (1988).

[46] Y. Seiya et al., Nucl. Instrum. Meth. A 480, 524 (2002).

[47] S. Bertolucci et al., Nucl. Instrum. Meth. A 267, 301 (1988).

[48] H.J. Frisch and P.J. Wilson, "Trigger Tower Organization and Summing in $\eta-\phi$ Space for Run II", CDF Internal Note 2045, 1993 (unpublished).

[49] P.J. Wilson, "Specification of Trigger Tower Summing and $E_{T}$ Weighting for ADMEM Boards", CDF Internal Note 4781, 1998 (unpublished).

[50] E.J. Thomson et al., IEEE Trans. Nucl. Sci. 49, 1063 (2002).

[51] M. Kruse, "Observation of Top Quark Pair Production in the Dilepton Decay Channel from Proton-Antiproton Collisions at $\sqrt{s}=1.8 \mathrm{TeV}$ ", Ph. D. thesis, Purdue University, 1996 (unpublished).

[52] F. Abe et al., Phys. Rev. Lett. 80, 2773 (1998).

[53] C. Miao, "Measurement of the $t \bar{t}$ Production Cross Section in the Lepton Plus Jets Cahannel in $p \bar{p}$ Collisions at $\sqrt{s}=1.8 \mathrm{TeV}$ ", Ph. D. thesis, University of Michigan, 1997 (unpublished). 
[54] D. Glenzinski, "Observation of the Top Quark in Proton-Antiproton Collisions at a Center of Mass Energy of 1.8 TeV", Ph. D. thesis, The Johns Hopkins University, 1995 (unpublished).

[55] H. Bachacou et al., "tt̄ Event Selection and Detection Efficiency for Winter 2003 Lepton+Jets Analysis", CDF Internal Note 6804, 2003 (unpublished).

[56] D. Acosta et al., "Measurement of the $\mathrm{t}$ anti-t production cross section in $\mathrm{p}$ anti$\mathrm{p}$ collisions at $\sqrt{s}=1.96-\mathrm{TeV}$ using kinematic fitting of b-tagged lepton + jet events", hep-ex/0409029.

[57] A. Taffard, "Run II Cosmic Ray Tagger", CDF Internal Note 6100, 2002 (unpublished).

[58] A. Taffard, "Run II Cosmic Ray Tagger Performances", CDF Internal Note 6255, 2003 (unpublished).

[59] T. Affolder et al., Phys. Rev. D 64, 032002 (2001).

[60] J-F. Arguin, B. Heinemann, "Underlying Event Corrections for Run II", CDF Internal Note 6239, 2003 (unpublished).

[61] S. Rappoccio, J. Guimaraes da Costa, "Impact of PrimeVtx on SecVtx For Summer 2003 Conferences", CDF Internal Note 6666, 2003 (unpublished).

[62] G. Marchesini and B. Webber, Nucl. Phys. B 310, 461 (1988).

[63] G. Corcella et al., JHEP 0101, 010 (2001).

[64] E. Brubaker, Ph.D. thesis, University of Caifornia, Berkeley, 2004 (unpublished).

[65] F. James and M. Roos., "MINUIT A System for Function Minimization and Analysis of the Parameter Errors and Correlations.", Comput. Phys. Commun., 10, 343 (1975).

[66] T. Sjöstrand et al., Comput. Phys. Commun. 135, 238 (2001).

[67] J. Pumplin et al., JHEP 0207, 012 (2002).

[68] P. Avery, K. Read and G. Trahern "QQ: A Monte Carlo Generator." CLEO internal report CSN 85/212, 1985, (unpublished).

[69] M.L. Mangano et al., "ALPGEN, a generator for hard multiparton processes in hadronic collisions", JHEP 0307:001, (2003); M.L. Mangano et al., "Multijet matrix elements and shower evolution in hadronic colllisions: W B Bbar $+\mathrm{N}$ jets as a case study", Nucl. Phys. B632:343-362 (2002); F. Caravaglios et al., "A new approach to multijet calculations in hadron collisions", Nucl. Phys. B539:215-232 (1999). 
[70] R. Brun and F. Carminati, CERN Programming Library Long Writeup W5013 (1993).

[71] R. Veenhof, CERN Programming Library Writeup W5050 (2001).

[72] G. Grindhammer, M. Rudowicz and S. Peters, Nucl. Instrum. and Methods A 290, 469 (1990).

[73] E. Gerchtein and M. Paulini, ECONF C0303241, TUMT005 (2003), arXiv:physics/0306031.

[74] F. Maltoni and T. Stelzer, hep-ph/0208156.

[75] T. Stelzer and W.F. Long, Phys. Commun. 81, 357-371 (1994).

[76] M. Jezabek and J.H. Kühn, Nucl. Phys. B314, 1 (1989).

[77] M. Fischer, S. Groote, J.G. Körner and M.C. Mauser, Phys. Lett. B451, 406 (1999).

[78] G. Feldman and R. Cousins, Phys. Rev. D 57, 3873 (1998).

[79] A.A. Bhatti and K. Hatakeyama, "Relative jet energy corrections using missing Et projection fraction and dijet balancing", CDF Internal Note 6854, 2004 (unpublished).

[80] J-F Arguin, et al., "Systematic Uncertainties Associated with Jet Corrections for Winter 2003 Conferences", CDF Note 6419, 2003 (unpublished).

[81] J-F Arguin, et al., "Jet Corrections for Summer 2003 Analyses (Offline Version 4.10.4)", CDF Note 6565, 2003 (unpublished).

[82] R.D. Field, See talks available from http://www.phys.ufl.edu/rfield/cdf/.

[83] http://durpdg.dur.ac.uk/hepdata/mrs.html.

[84] K. Bloom, et al., "Measurement of $\mathrm{B}(\mathrm{t} \rightarrow \mathrm{Wb}) / \mathrm{B}(\mathrm{t} \rightarrow \mathrm{Wq})$ in the SECVTXTagged W+Jets Sample", CDF Note 6822, 2004 (unpublished).

[85] K. Bloom, et al., "SVX-Tagging Efficiency for $t \bar{t}$ Dilepton and Lepton-Plus-Jets Events", CDF Note 7070, 2004 (unpublished).

[86] J. Bialek et al., "COT Wireplane Testing at the University of Illinois", CDF Internal Note 4570, 1998 (unpublished).

[87] G. Ascoli et al., Nuclear Instruments and Methods, A268 33 (1988).

[88] LabWindows is a registered trademark of National Instruments.

[89] Kapton is a registered trademark of DuPont Teijin Films. 
[90] D. Khazins, B. Wagner and I. Yu, "Aging Test for COT", CDF Internal Note 3894, 1996 (unpublished).

[91] G. Brandenburg et al., "The CDF Run 1 Muon System Upgrade", CDF Internal Note 6362, 2003 (unpublished).

[92] Glasteel is a trademark of Glasteel Industrial Laminates, Colliverville, TN.

[93] K. Lannon, Ph.D. thesis, University of Illinois at Urbana-Champaign, 2003 (unpublished).

[94] B. Kilminster et al., "Search for non-standard model couplings in top decay", CDF note 6235, 2002 (unpublished).

[95] H. Murayama, I. Watanabe, K. Hagiwara, "HELAS: HELICITY AMPLITUDE SUBROUTINES FOR FEYNMAN DIAGRAM EVALUATIONS", KEK-91-11, 184 (1992).

[96] R. A. Fisher and F. Yates, Statistical Tables, Example 12, London (1938).

[97] D. E. Knuth, The Art of Computer Programming, Vol. 2, Third Edition, Section 3.4.2, Algorithm P, pp 145, Addison-Wesley (1997).

[98] S. Mrenna, "Generator Resource and Information Webpage", http://wwwcpd.fnal.gov/personal/mrenna/generator.html.

[99] C. Campagnari and M. Franklin, Rev. Mod. Phys. 69, 137 (1997).

[100] G. Apollinari et al., "New determination of SECVTX and JPB mistag rates using jet data", CDF Internal Note 3990, 1998 (unpublished). 


\section{Author's Biography}

Trevor Neil Vickey was born in Waukegan, Illinois on February 4, 1975. As a child he was very curious about the world around him, asking his parents and teachers endless strings of questions. He had a chemistry set and a microscope. He enjoyed building and launching model rockets, some of which contained payloads of worms and toads which thankfully always returned alive. During his early years of high school he dreamt of becoming an architect, drafted floor-plans and built balsa-wood models. A school field-trip to an architecture firm proved to be so boring, that he resolved to find something much more interesting and challenging to do with his life. He thoroughly enjoyed his high-school physics course and decided to pursue a degree in Physics. As an undergraduate physics major he visited the CDF experiment during a class visit to Fermilab, but never imagined then that it would one day become his thesis experiment. He received a B.S. in Physics from the University of Illinois at UrbanaChampaign in 1998 and decided to remain in Urbana for graduate study. He joined the CDF experiment in 1999, and after studying top quark physics, graduated with a Ph.D. in Physics in 2004. He accepted a position as a postdoctoral research assistant

with the University of Wisconsin, Madison to work on the ATLAS experiment at CERN and search for the elusive Higgs boson. 